\title{
A Search for the Production of Technicolor Particles at the $\mathrm{D} \varnothing$ Detector
}

\author{
A Dissertation Presented \\ by \\ Satish Vijay Desai \\ to \\ The Graduate School \\ in Partial Fulfillment of the
}

Requirements

for the Degree of

Doctor of Philosophy

in

Physics and Astronomy

Stony Brook University

August 2006 
Copyright by

Satish Vijay Desai 2006 


\section{State University of New York at Stony Brook \\ The Graduate School \\ Satish Vijay Desai}

We, the dissertation committee for the above candidate for the Doctor of Philosophy degree, hereby recommend acceptance of the dissertation.

\begin{tabular}{c}
\hline $\begin{array}{c}\text { Professor John Hobbs } \\
\text { Advisor }\end{array}$ \\
\hline $\begin{array}{c}\text { Professor George Sterman } \\
\text { Chair of the defense }\end{array}$ \\
\hline Professor Thomas Hemmick \\
\hline Professor Anna Goussiou \\
Outside member, University of Notre Dame
\end{tabular}

This dissertation is accepted by the Graduate School.

Graduate School 


\title{
Abstract of the Dissertation A Search for the Production of Technicolor Particles at the $\mathrm{D} \varnothing$ Detector
}

\author{
by \\ Satish Vijay Desai \\ Doctor of Philosophy \\ in \\ Physics and Astronomy \\ Stony Brook University \\ John D. Hobbs, Advisor
}

2006

\begin{abstract}
A search for the Technicolor processes $p \bar{p} \rightarrow \rho_{T}^{ \pm} \rightarrow W^{ \pm} \pi_{T}^{0} \rightarrow$ $\mu \nu b \bar{b}$ and $p \bar{p} \rightarrow \rho_{T}^{0} \rightarrow W^{ \pm} \pi_{T}^{\mp} \rightarrow \mu \nu b \bar{c}$ is conducted at the $\mathrm{D} \varnothing$ detector. Selection requirements are individually optimized for each of twenty mass hypotheses by means of a random grid search. No excess is seen in a $291 \mathrm{pb}^{-1}$ data set and $95 \%$ confidence level upper limits are set on the Technicolor production cross section. The mass combinations $M_{\rho}=195 \mathrm{GeV} / c^{2}, M_{\pi}=100 \mathrm{GeV} / c^{2}$ and $M_{\rho}=200 \mathrm{GeV} / c^{2}, M_{\pi}=105 \mathrm{GeV} / c^{2}$ are excluded for the choice of the Technicolor scale parameter $M_{V}=500 \mathrm{GeV}$.
\end{abstract}


Dedicated to my parents: with love to my mother, and in remembrance of my father. 
'What more do you want to know?'

'The names of all the stars, and of all living things, and the whole history of Middle-earth and Over-heaven and of the Sundering Seas,' laughed Pippin. 'Of course! What less?'

- J.R.R. Tolkien, The Two Towers. 


\section{Contents}

Acknowledgements $\quad$ xiv

1 Introduction $\quad 1$

1.1 Electroweak Unification . . . . . . . . . . . . . . . 3

1.2 The Higgs Mechanism . . . . . . . . . . . . . . . 6

1.3 An Alternative Solution - Technicolor . . . . . . . . . . 8

1.3.1 Extensions to Technicolor . . . . . . . . . . . . . . 10

1.3.2 Low Scale Technicolor Phenomenology . . . . . . . . . 12

1.4 Previous Searches . . . . . . . . . . . . . . 13

1.5 Searching for Technicolor at DØ Run II . . . . . . . . . . . . . 17

2 Experimental Apparatus $\quad 20$

2.1 The Tevatron Accelerator Complex . . . . . . . . . . . . . . 20

2.2 The D $\varnothing$ Coordinate System . . . . . . . . . . . . . . . . 21

2.3 The DØ Detector . . . . . . . . . . . . . . . . 22

2.3.1 The Silicon Microstrip Tracker . . . . . . . . . . 24

2.3.2 The Central Fiber Tracker . . . . . . . . . . . . 26

2.3.3 The Central and Forward Preshowers . . . . . . . . . . 27

2.3.4 The Solenoid . . . . . . . . . . . . . . . . 29

2.3.5 The Calorimeter . . . . . . . . . . . . 30

2.3.6 The Muon System . . . . . . . . . . . . . . . 32

2.3.7 The Trigger and Data Acquisition Systems . . . . . . 35

3 Event Reconstruction 38

3.1 Track Reconstruction . . . . . . . . . . . . . . . . . 38

3.1.1 The Alternative Algorithm . . . . . . . . . . . . . . 39

3.1.2 The Histogramming Track Finder . . . . . . . . . . . . 40

3.1.3 Track Selection . . . . . . . . . . . . . . . . 40

3.2 Vertex Reconstruction . . . . . . . . . . . . . . . . . 42

3.2.1 Vertex Fitting . . . . . . . . . . . . . . . . 44

3.3 Jet Reconstruction . . . . . . . . . . . . . . . . . 45 
3.3.1 Jet Energy Scale . . . . . . . . . . . . . . . . . . 49

3.4 Muon Reconstruction . . . . . . . . . . . . . . . . . . . . 54

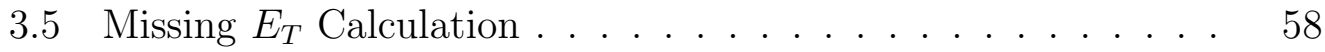

3.6 Electron and Photon Reconstruction . . . . . . . . . . 59

4 Event Simulation $\quad 61$

4.1 Simulation Software . . . . . . . . . . . . . . . . . . 61

4.2 Simulation Corrections . . . . . . . . . . . . . . . . 63

4.2 .1 Resolution Corrections . . . . . . . . . . . . . 63

4.2 .2 Efficiency Scale Factors . . . . . . . . . . . . . . . . 64

5 Heavy Quark Identification $\quad 67$

5.1 Track Jets and Taggability . . . . . . . . . . . . . . . . 68

$5.2 V^{0}$ Rejection . . . . . . . . . . . . . . . . . 68

5.3 The JLIP Tagging Algorithm . . . . . . . . . . . . . 70

5.4 The JLIP Tag Rate Functions . . . . . . . . . . . . . . . . 72

5.4.1 Evaluation of the Mistag Rate . . . . . . . . . . . 72

5.4.2 Determination of the Tagging Efficiencies . . . . . . 76

6 Event Selection $\quad 83$

6.1 Data Samples . . . . . . . . . . . . . . . . . . . . . 83

6.1.1 Simulated Cross Sections . . . . . . . . . . . . . 88

6.2 Selection of the $W b j \rightarrow \mu \nu b j$ Event Sample . . . . . . . . . 89

6.2.1 Normalization Procedure . . . . . . . . . . . . . . 94

6.3 Estimation of the Instrumental Background . . . . . . . . 100

6.4 Final Event Selection . . . . . . . . . . . . . . . . . . . . 101

6.4.1 Optimized Rejection of the Non- $W / Z$ Backgrounds . . 103

6.4.2 The Random Grid Search Technique . . . . . . . . . 104

7 Analysis and Results $\quad 110$

7.1 Systematic Uncertainties . . . . . . . . . . . . . . . . 110

7.2 Limit Setting Technique . . . . . . . . . . . . . . . . . . 112

7.3 Limits on Technicolor Production . . . . . . . . . . . . 113

8 Conclusions and Outlook 117

8.1 Other Recent Technicolor Searches . . . . . . . . . . . . . 117

8.2 Prospects for Improvement . . . . . . . . . . . . . . . . . . . . . 119

8.3 Further Ahead . . . . . . . . . . . . . . . . . . . . . . 121

$\begin{array}{ll}\text { A Optimization Refinement Figures } & 122\end{array}$ 
$\begin{array}{ll}\text { B Summaries of Event Yields } & 143\end{array}$

$\begin{array}{ll}\text { C Cut Variable Distributions } & 164\end{array}$

$\begin{array}{ll}\text { D Systematic Uncertainties } & 185\end{array}$

$\begin{array}{ll}\text { Bibliography } & 206\end{array}$ 


\section{List of Figures}

1.1 Feynman diagram for the Fermi theory of $\beta$-decay . . . . . . . 3

1.2 Feynman diagram for SM theory of $\beta$-decay . . . . . . . . . 4

1.3 $\mathrm{CDF} \rho_{T} \rightarrow W \pi_{T}$ exclusion region . . . . . . . . . . . . 16

$1.4 \mathrm{CDF} \omega_{T} \rightarrow \gamma \pi_{T} \rightarrow \gamma b \bar{b}$ exclusion region . . . . . . . . . . . 17

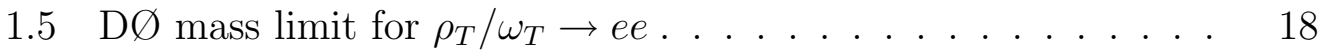

1.6 Feynman diagrams for $\rho_{T} \rightarrow W \pi_{T}$ at the Tevatron . . . . . . . 19

2.1 The Tevatron Accelerator Complex . . . . . . . . . . . . . . . 21

2.2 The $\mathrm{D} \varnothing$ inner tracking system . . . . . . . . . . . . . . . . . . . . . 22

2.3 Overview of the $\mathrm{D} \varnothing$ detector . . . . . . . . . . . . . 23

2.4 The Silicon Microstrip Tracker . . . . . . . . . . . . . . . 24

2.5 Schematic of the SMT readout . . . . . . . . . . . . 25

2.6 The central fiber tracker . . . . . . . . . . . . . . 26

2.7 Prehsower scintillating strip cross sections . . . . . . . . . . 28

2.8 FPS MIP and shower layer modules . . . . . . . . . . . . . 29

2.9 The $\mathrm{D} \varnothing$ calorimeter . . . . . . . . . . . . . . . . . . . 30

2.10 Calorimeter cell schematic . . . . . . . . . . . . . . 31

2.11 Cross-section D $\varnothing$ calorimeter . . . . . . . . . . . . . 32

2.12 The $\mathrm{D} \varnothing$ muon drift tubes . . . . . . . . . . . . . . . . . . . . . . . . . . . . . . . . . 33

2.13 The $\mathrm{D} \varnothing$ muon scintillators . . . . . . . . . . . . . . . . 33

3.1 HTF reconstruction example . . . . . . . . . . . . . . . . . . 41

3.2 Distribution of $\log _{10}\left(p_{T}\right)$ for min. bias tracks . . . . . . . . . . 44

3.3 Infrared problems in jet reconstruction . . . . . . . . . . . . . 46

3.4 Effects of parton splitting on jet reconstruction . . . . . . . . 46

3.5 Dangers of $p_{T}$-orded jet reconstruction seeds . . . . . . . . . . 47

3.6 Jet quality distributions . . . . . . . . . . . . . . . 50

3.7 Ratio of L1 to reconstructed jet energies . . . . . . . . . . . 51

3.8 Jet correction factors and uncertainties for data . . . . . . . . 52

3.9 Jet correction factors and uncertainties in the simulation . . . 53

3.10 Muon trajectory in a drift chamber . . . . . . . . . . . 55 
3.11 Missing $E_{T}$ in $Z \rightarrow \mu \mu$ events $\ldots \ldots \ldots \ldots \ldots$

4.1 Distributions of $\mathbb{E}_{T}$ components in $Z \rightarrow \mu \mu$ events $\ldots \ldots 6$

5.1 Invariant $\pi^{+} \pi^{-}$and $p \pi^{-}$mass distributions . . . . . . . . 69

5.2 Track impact parameter significance distributions . . . . . . 71

$5.3 \mathcal{P}_{\text {trk }}$ distributions in jet-trigger and simulated data . . . . . . 73

$5.4 \mathcal{P}_{\text {jet }}$ distributions in jet-trigger and simulated data . . . . . 74

5.5 JLIP mistag rates . . . . . . . . . . . . . . . . 75

5.6 Simulated tagging efficiencies for $b$ - and $c$-jets $\ldots \ldots \ldots 77$

5.7 Tag rate function for $b$-jets . . . . . . . . . . . . 80

5.8 Tag rate function for $c$-jets . . . . . . . . . . . 81

6.1 Technicolor mass grid and cross sections $\ldots \ldots \ldots \ldots$

6.2 Muon $p_{T}$ spectra . . . . . . . . . . . . . . . . . . 91

6.3 Missing $E_{T}$ distributions . . . . . . . . . . . . . . . 94

$6.4 W$ transverse mass distributions . . . . . . . . . . . . . 95

6.5 Jet multiplicity after $W$ selection $\ldots \ldots \ldots$. . . . . . . 96

6.6 Jet $p_{T}$ and $\eta$ distributions $\ldots \ldots \ldots \ldots$. . . . . . . . . . . 97

6.7 Properties of the dijet system . . . . . . . . . . . . . . 98

6.8 Properties of the $b-j$ system $\ldots \ldots \ldots \ldots$

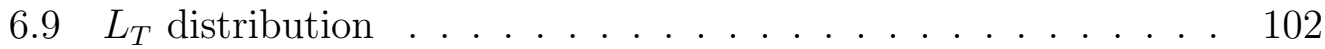

6.10 Two dimensional $L_{T}-p_{T}^{j j}$ distributions . . . . . . . . . . 104

6.11 Optimized selection requirements . . . . . . . . . . . 107

6.12 Marginal significances for the random grid search . . . . . . 108

6.13 Final event yields . . . . . . . . . . . . . . . . . . . . 109

7.1 Cross sections and limits for $M_{V}=100 \mathrm{GeV} \ldots \ldots \ldots$

7.2 Cross sections and limits for $M_{V}=500 \mathrm{GeV} \ldots \ldots \ldots$

8.1 Electron channel neural net exclusion region . . . . . . . . 120

A.1 Selection refinement for $M_{\rho}=175 \mathrm{GeV} / c^{2}, M_{\pi}=90 \mathrm{GeV} / c^{2}$. 123

A.2 Selection refinement for $M_{\rho}=180 \mathrm{GeV} / c^{2}, M_{\pi}=100 \mathrm{GeV} / c^{2} \quad 124$

A.3 Selection refinement for $M_{\rho}=190 \mathrm{GeV} / c^{2}, M_{\pi}=100 \mathrm{GeV} / c^{2} \quad 125$

A.4 Selection refinement for $M_{\rho}=190 \mathrm{GeV} / c^{2}, M_{\pi}=110 \mathrm{GeV} / c^{2} \quad 126$

A.5 Selection refinement for $M_{\rho}=195 \mathrm{GeV} / c^{2}, M_{\pi}=100 \mathrm{GeV} / c^{2} \quad 127$

A.6 Selection refinement for $M_{\rho}=200 \mathrm{GeV} / c^{2}, M_{\pi}=105 \mathrm{GeV} / c^{2} \quad 128$

A.7 Selection refinement for $M_{\rho}=200 \mathrm{GeV} / c^{2}, M_{\pi}=120 \mathrm{GeV} / c^{2} \quad 129$

A.8 Selection refinement for $M_{\rho}=210 \mathrm{GeV} / c^{2}, M_{\pi}=110 \mathrm{GeV} / c^{2} \quad 130$

A.9 Selection refinement for $M_{\rho}=215 \mathrm{GeV} / c^{2}, M_{\pi}=130 \mathrm{GeV} / c^{2} \quad 131$ 
A.10 Selection refinement for $M_{\rho}=220 \mathrm{GeV} / c^{2}, M_{\pi}=120 \mathrm{GeV} / c^{2} \quad 132$

A.11 Selection refinement for $M_{\rho}=220 \mathrm{GeV} / c^{2}, M_{\pi}=140 \mathrm{GeV} / c^{2} \quad 133$

A.12 Selection refinement for $M_{\rho}=235 \mathrm{GeV} / c^{2}, M_{\pi}=120 \mathrm{GeV} / c^{2} 134$

A.13 Selection refinement for $M_{\rho}=235 \mathrm{GeV} / c^{2}, M_{\pi}=130 \mathrm{GeV} / c^{2} \quad 135$

A.14 Selection refinement for $M_{\rho}=235 \mathrm{GeV} / c^{2}, M_{\pi}=150 \mathrm{GeV} / c^{2} \quad 136$

A.15 Selection refinement for $M_{\rho}=240 \mathrm{GeV} / c^{2}, M_{\pi}=140 \mathrm{GeV} / c^{2} \quad 137$

A.16 Selection refinement for $M_{\rho}=250 \mathrm{GeV} / c^{2}, M_{\pi}=130 \mathrm{GeV} / c^{2} \quad 138$

A.17 Selection refinement for $M_{\rho}=255 \mathrm{GeV} / c^{2}, M_{\pi}=130 \mathrm{GeV} / c^{2} \quad 139$

A.18 Selection refinement for $M_{\rho}=255 \mathrm{GeV} / c^{2}, M_{\pi}=150 \mathrm{GeV} / c^{2} \quad 140$

A.19 Selection refinement for $M_{\rho}=260 \mathrm{GeV} / c^{2}, M_{\pi}=140 \mathrm{GeV} / c^{2} \quad 141$

A.20 Selection refinement for $M_{\rho}=275 \mathrm{GeV} / c^{2}, M_{\pi}=150 \mathrm{GeV} / c^{2} 142$

C.1 Distributions for $M_{\rho}=175 \mathrm{GeV} / c^{2}, M_{\pi}=90 \mathrm{GeV} / c^{2}$. . . . . 165

C.2 Distributions for $M_{\rho}=180 \mathrm{GeV} / c^{2}, M_{\pi}=100 \mathrm{GeV} / c^{2}$. . . . 166

C.3 Distributions for $M_{\rho}=190 \mathrm{GeV} / c^{2}, M_{\pi}=100 \mathrm{GeV} / c^{2}$. . . . 167

C.4 Distributions for $M_{\rho}=190 \mathrm{GeV} / c^{2}, M_{\pi}=110 \mathrm{GeV} / c^{2}$. . . . 168

C.5 Distributions for $M_{\rho}=195 \mathrm{GeV} / c^{2}, M_{\pi}=100 \mathrm{GeV} / c^{2}$. . . . 169

C.6 Distributions for $M_{\rho}=200 \mathrm{GeV} / c^{2}, M_{\pi}=105 \mathrm{GeV} / c^{2}$. . . . 170

C.7 Distributions for $M_{\rho}=200 \mathrm{GeV} / c^{2}, M_{\pi}=120 \mathrm{GeV} / c^{2}$. . . . 171

C.8 Distributions for $M_{\rho}=210 \mathrm{GeV} / c^{2}, M_{\pi}=110 \mathrm{GeV} / c^{2}$. . . . 172

C.9 Distributions for $M_{\rho}=215 \mathrm{GeV} / c^{2}, M_{\pi}=130 \mathrm{GeV} / c^{2}$. . . . 173

C.10 Distributions for $M_{\rho}=220 \mathrm{GeV} / c^{2}, M_{\pi}=120 \mathrm{GeV} / c^{2}$. . . . 174

C.11 Distributions for $M_{\rho}=220 \mathrm{GeV} / c^{2}, M_{\pi}=140 \mathrm{GeV} / c^{2}$. . . . 175

C.12 Distributions for $M_{\rho}=235 \mathrm{GeV} / c^{2}, M_{\pi}=120 \mathrm{GeV} / c^{2} \ldots$. . 176

C.13 Distributions for $M_{\rho}=235 \mathrm{GeV} / c^{2}, M_{\pi}=130 \mathrm{GeV} / c^{2} \ldots$. . . 177

C.14 Distributions for $M_{\rho}=235 \mathrm{GeV} / c^{2}, M_{\pi}=150 \mathrm{GeV} / c^{2} \ldots$. . 178

C.15 Distributions for $M_{\rho}=240 \mathrm{GeV} / c^{2}, M_{\pi}=140 \mathrm{GeV} / c^{2} \ldots$. . 179

C.16 Distributions for $M_{\rho}=250 \mathrm{GeV} / c^{2}, M_{\pi}=130 \mathrm{GeV} / c^{2}$. . . . 180

C.17 Distributions for $M_{\rho}=255 \mathrm{GeV} / c^{2}, M_{\pi}=130 \mathrm{GeV} / c^{2} \ldots$. . . 181

C.18 Distributions for $M_{\rho}=255 \mathrm{GeV} / c^{2}, M_{\pi}=150 \mathrm{GeV} / c^{2}$. . . . 182

C.19 Distributions for $M_{\rho}=260 \mathrm{GeV} / c^{2}, M_{\pi}=140 \mathrm{GeV} / c^{2}$. . . . 183

C.20 Distributions for $M_{\rho}=275 \mathrm{GeV} / c^{2}, M_{\pi}=150 \mathrm{GeV} / c^{2} \ldots$. . 184 


\section{List of Tables}

1.1 Electroweak quantum numbers for fermions . . . . . . . . 5

1.2 Sample decay rates and coefficients for $V_{T} \rightarrow G \pi_{T} \ldots$. . . . . 14

2.1 Calorimeter cell composition ............. . . 31

3.1 Track requirements for vertex reconstruction . . . . . . . . . . 43

3.2 Reconstructed muon categories . . . . . . . . . . . . 56

4.1 Jet momentum resolution parameters . . . . . . . . . . . . . . 64

4.2 Jet reconstruction scale-factor parameters . . . . . . . . 66

5.1 Mistag rate fit-parameters . . . . . . . . . . . . 76

5.2 Tag rate fit-parameters for $b$ - and $c$-jets . . . . . . . . . 82

6.1 Generated Technicolor samples . . . . . . . . . . . . 86

6.2 Simulated background samples . . . . . . . . . . . . . . 87

6.3 Additional diboson backgrounds . . . . . . . . . . . . . . . . 88

6.4 Standard Model and data event yields after initial selection . . 92

6.5 Technicolor event yields after initial selection . . . . . . . . . . 93

6.6 Muon isolation efficiencies . . . . . . . . . . . . . . . . . 101

6.7 Optimzed selection requirements . . . . . . . . . 106

7.1 Background systematics during the initial selection . . . . . . 111

7.2 Results for $M_{V}=100 \mathrm{GeV}$. . . . . . . . . . . . . . . 114

7.3 Results for $M_{V}=500 \mathrm{GeV}$. . . . . . . . . . . . 115

8.1 Comparison of the electron and muon search results . . . . . . 118

8.2 Electron channel exclusion region . . . . . . . . . . . . . 118

B.1 Event yields for $M_{\rho}=175 \mathrm{GeV} / c^{2}, M_{\pi}=90 \mathrm{GeV} / c^{2}$. . . 144

B.2 Event yields for $M_{\rho}=180 \mathrm{GeV} / c^{2}, M_{\pi}=100 \mathrm{GeV} / c^{2}$. . . 145

B.3 Event yields for $M_{\rho}=190 \mathrm{GeV} / c^{2}, M_{\pi}=100 \mathrm{GeV} / c^{2}$. . 146

B.4 Event yields for $M_{\rho}=190 \mathrm{GeV} / c^{2}, M_{\pi}=110 \mathrm{GeV} / c^{2}$. . 147 
B.5 Event yields for $M_{\rho}=195 \mathrm{GeV} / c^{2}, M_{\pi}=100 \mathrm{GeV} / c^{2} \ldots 148$

B.6 Event yields for $M_{\rho}=200 \mathrm{GeV} / c^{2}, M_{\pi}=105 \mathrm{GeV} / c^{2} \ldots 149$

B.7 Event yields for $M_{\rho}=200 \mathrm{GeV} / c^{2}, M_{\pi}=120 \mathrm{GeV} / c^{2} \ldots 150$

B.8 Event yields for $M_{\rho}=210 \mathrm{GeV} / c^{2}, M_{\pi}=110 \mathrm{GeV} / c^{2} \ldots 151$

B.9 Event yields for $M_{\rho}=215 \mathrm{GeV} / c^{2}, M_{\pi}=130 \mathrm{GeV} / c^{2} \ldots 152$

B.10 Event yields for $M_{\rho}=220 \mathrm{GeV} / c^{2}, M_{\pi}=120 \mathrm{GeV} / c^{2} \ldots 153$

B.11 Event yields for $M_{\rho}=220 \mathrm{GeV} / c^{2}, M_{\pi}=140 \mathrm{GeV} / c^{2} \ldots 154$

B.12 Event yields for $M_{\rho}=235 \mathrm{GeV} / c^{2}, M_{\pi}=120 \mathrm{GeV} / c^{2} \ldots 155$

B.13 Event yields for $M_{\rho}=235 \mathrm{GeV} / c^{2}, M_{\pi}=130 \mathrm{GeV} / c^{2} \ldots 156$

B.14 Event yields for $M_{\rho}=235 \mathrm{GeV} / c^{2}, M_{\pi}=150 \mathrm{GeV} / c^{2} \ldots 157$

B.15 Event yields for $M_{\rho}=240 \mathrm{GeV} / c^{2}, M_{\pi}=140 \mathrm{GeV} / c^{2} \ldots 158$

B.16 Event yields for $M_{\rho}=250 \mathrm{GeV} / c^{2}, M_{\pi}=130 \mathrm{GeV} / c^{2} \ldots 159$

B.17 Event yields for $M_{\rho}=255 \mathrm{GeV} / c^{2}, M_{\pi}=130 \mathrm{GeV} / c^{2} \ldots 160$

B.18 Event yields for $M_{\rho}=255 \mathrm{GeV} / c^{2}, M_{\pi}=150 \mathrm{GeV} / c^{2} \ldots 161$

B.19 Event yields for $M_{\rho}=260 \mathrm{GeV} / c^{2}, M_{\pi}=140 \mathrm{GeV} / c^{2} \ldots 162$

B.20 Event yields for $M_{\rho}=275 \mathrm{GeV} / c^{2}, M_{\pi}=150 \mathrm{GeV} / c^{2} \ldots 163$

D.1 Background errors for $M_{\rho}=175 \mathrm{GeV} / c^{2}, M_{\pi}=90 \mathrm{GeV} / c^{2} \quad 186$

D.2 Signal errors for $M_{\rho}=175 \mathrm{GeV} / c^{2}, M_{\pi}=90 \mathrm{GeV} / c^{2} \ldots 186$

D.3 Background errors for $M_{\rho}=180 \mathrm{GeV} / c^{2}, M_{\pi}=100 \mathrm{GeV} / c^{2} 187$

D.4 Signal errors for $M_{\rho}=180 \mathrm{GeV} / c^{2}, M_{\pi}=100 \mathrm{GeV} / c^{2} \ldots 187$

D.5 Background errors for $M_{\rho}=190 \mathrm{GeV} / c^{2}, M_{\pi}=100 \mathrm{GeV} / c^{2} 188$

D.6 Signal errors for $M_{\rho}=190 \mathrm{GeV} / c^{2}, M_{\pi}=100 \mathrm{GeV} / c^{2} \ldots 188$

D.7 Background errors for $M_{\rho}=190 \mathrm{GeV} / c^{2}, M_{\pi}=110 \mathrm{GeV} / c^{2} 189$

D.8 Signal errors for $M_{\rho}=190 \mathrm{GeV} / c^{2}, M_{\pi}=110 \mathrm{GeV} / c^{2} \ldots 189$

D.9 Background errors for $M_{\rho}=195 \mathrm{GeV} / c^{2}, M_{\pi}=100 \mathrm{GeV} / c^{2} 190$

D.10 Signal errors for $M_{\rho}=195 \mathrm{GeV} / c^{2}, M_{\pi}=100 \mathrm{GeV} / c^{2} \ldots 190$

D.11 Background errors for $M_{\rho}=200 \mathrm{GeV} / c^{2}, M_{\pi}=105 \mathrm{GeV} / c^{2} 191$

D.12 Signal errors for $M_{\rho}=200 \mathrm{GeV} / c^{2}, M_{\pi}=105 \mathrm{GeV} / c^{2} \ldots 191$

D.13 Background errors for $M_{\rho}=200 \mathrm{GeV} / c^{2}, M_{\pi}=120 \mathrm{GeV} / c^{2} 192$

D.14 Signal errors for $M_{\rho}=200 \mathrm{GeV} / c^{2}, M_{\pi}=120 \mathrm{GeV} / c^{2} \ldots 192$

D.15 Background errors for $M_{\rho}=210 \mathrm{GeV} / c^{2}, M_{\pi}=110 \mathrm{GeV} / c^{2} 193$

D.16 Signal errors for $M_{\rho}=210 \mathrm{GeV} / c^{2}, M_{\pi}=110 \mathrm{GeV} / c^{2} \ldots 193$

D.17 Background errors for $M_{\rho}=215 \mathrm{GeV} / c^{2}, M_{\pi}=130 \mathrm{GeV} / c^{2} 194$

D.18 Signal errors for $M_{\rho}=215 \mathrm{GeV} / c^{2}, M_{\pi}=130 \mathrm{GeV} / c^{2} \ldots 194$

D.19 Background errors for $M_{\rho}=220 \mathrm{GeV} / c^{2}, M_{\pi}=120 \mathrm{GeV} / c^{2} 195$

D.20 Signal errors for $M_{\rho}=220 \mathrm{GeV} / c^{2}, M_{\pi}=120 \mathrm{GeV} / c^{2} \ldots 195$

D.21 Background errors for $M_{\rho}=220 \mathrm{GeV} / c^{2}, M_{\pi}=140 \mathrm{GeV} / c^{2} 196$

D.22 Signal errors for $M_{\rho}=220 \mathrm{GeV} / c^{2}, M_{\pi}=140 \mathrm{GeV} / c^{2} \ldots 196$

D.23 Background errors for $M_{\rho}=235 \mathrm{GeV} / c^{2}, M_{\pi}=120 \mathrm{GeV} / c^{2} 197$ 
D.24 Signal errors for $M_{\rho}=235 \mathrm{GeV} / c^{2}, M_{\pi}=120 \mathrm{GeV} / c^{2}$. . . 197

D.25 Background errors for $M_{\rho}=235 \mathrm{GeV} / c^{2}, M_{\pi}=130 \mathrm{GeV} / c^{2} 198$

D.26 Signal errors for $M_{\rho}=235 \mathrm{GeV} / c^{2}, M_{\pi}=130 \mathrm{GeV} / c^{2}$. . . 198

D.27 Background errors for $M_{\rho}=235 \mathrm{GeV} / c^{2}, M_{\pi}=150 \mathrm{GeV} / c^{2} 199$

D.28 Signal errors for $M_{\rho}=235 \mathrm{GeV} / c^{2}, M_{\pi}=150 \mathrm{GeV} / c^{2}$. . . 199

D.29 Background errors for $M_{\rho}=240 \mathrm{GeV} / c^{2}, M_{\pi}=140 \mathrm{GeV} / c^{2} 200$

D.30 Signal errors for $M_{\rho}=240 \mathrm{GeV} / c^{2}, M_{\pi}=140 \mathrm{GeV} / c^{2}$. . . 200

D.31 Background errors for $M_{\rho}=250 \mathrm{GeV} / c^{2}, M_{\pi}=130 \mathrm{GeV} / c^{2} 201$

D.32 Signal errors for $M_{\rho}=250 \mathrm{GeV} / c^{2}, M_{\pi}=130 \mathrm{GeV} / c^{2}$. . . 201

D.33 Background errors for $M_{\rho}=255 \mathrm{GeV} / c^{2}, M_{\pi}=130 \mathrm{GeV} / c^{2} 202$

D.34 Signal errors for $M_{\rho}=255 \mathrm{GeV} / c^{2}, M_{\pi}=130 \mathrm{GeV} / c^{2}$. . . 202

D.35 Background errors for $M_{\rho}=255 \mathrm{GeV} / c^{2}, M_{\pi}=150 \mathrm{GeV} / c^{2} 203$

D.36 Signal errors for $M_{\rho}=255 \mathrm{GeV} / c^{2}, M_{\pi}=150 \mathrm{GeV} / c^{2}$. . . 203

D.37 Background errors for $M_{\rho}=260 \mathrm{GeV} / c^{2}, M_{\pi}=140 \mathrm{GeV} / c^{2} 204$

D.38 Signal errors for $M_{\rho}=260 \mathrm{GeV} / c^{2}, M_{\pi}=140 \mathrm{GeV} / c^{2}$. . . 204

D.39 Background errors for $M_{\rho}=275 \mathrm{GeV} / c^{2}, M_{\pi}=150 \mathrm{GeV} / c^{2} 205$

D.40 Signal errors for $M_{\rho}=275 \mathrm{GeV} / c^{2}, M_{\pi}=150 \mathrm{GeV} / c^{2}$. . . 205 


\section{Acknowledgements}

The work of science has always been a collaborative effort, but in the field of particle physics this is especially true. This dissertation would not have been possible without the contributions, technical and otherwise, of many people.

I am deeply indebted to John Hobbs, my dissertation advisor. The death of my father demanded a great deal of my attention for an extended period of time. John's efforts allowed me to continue work on my degree and made a difficult time far easier. However, he has earned my gratitude in the more usual aspects of his role as well. John has given me significant freedom to pursue my own contributions to D $\varnothing$, and has been very supportive when I transitioned to doing analysis. His focus on the end result has also been valuable to someone who has a tendency to pursue tangents too far.

For their contributions to the development of this analysis, I am especially grateful to Peter Tamburello, Lorenzo Feligioni, and Meenakshi Narain. Much of my analysis software was adapted from a Standard Model Higgs search by Peter. The ways in which my work depends on his are too numerous to list, so I shall not try. Lorenzo and Meenakshi conducted a Technicolor search in parallel with my own. We faced common issues and their input has been extremely useful.

I owe a great deal to the administrative staff at Stony Brook. Pat Peiliker is essential to the Physics and Astronomy Department. Likewise, Joan Napolitano and Alice Dugan are vital to the high energy group. Furthermore, Joan and Pat worked with John to develop an arrangement by which I could continue to work part-time toward my Ph.D. while managing the sale of my father's estate. Without their efforts, these circumstances could have proven disastrous.

I am privileged to have many good friends at Fermilab. I've shared good times (and some tough ones) with Andrew Askew, Angela Bellavance, Greg Davis, Mike Cooke, Robert Illingworth, and Ike and Heather Hall. Most of these are also my colleagues, and I am grateful to them both professionally and personally. As a house-sitter, and later as a housemate, Danielle Albright has helped preserve some measure of sanity at home. As a friend, she has been 
a welcome source of distraction and entertainment.

Matthew Malek and Lisa Makros I have known almost as long as I have been in grad school. I met Pam Williams and Craig Counterman a few years later. These are my dearest friends, my family. They have all lent me strength and support that has been of immeasurable value. However, I must single out Matthew, who also helped me to edit this dissertation.

My sister, Angela, has often seemed to be more my twin than my younger sibling. I am very fortunate indeed to be her brother. To my parents, I will be eternally grateful. Both, I know, have worked hard over the years to give me the encouragement to achieve whatever I wanted to achieve. My mother, Roni, I thank for teaching me that understanding the subject was more important than the best grades. My father, Vijay, I thank for raising me as a scientist. I know he was always very proud of me, and I deeply wish he could be here to witness the completion of my degree. 


\section{Chapter 1}

\section{Introduction}

The twentieth century has witnessed the construction of a comprehensive theory of the fundamental structure of matter. The Standard Model of particle physics combines special relativity with quantum mechanics to describe the world at the smallest distance scales currently accessible to experiment. It has withstood nearly all tests with astonishing resiliency. Only the recent demonstration of neutrino oscillations has disagreed with it. ${ }^{1}$

The familiar subatomic particles, the electron, proton and neutron, are fermions: they have an intrinsic angular momentum (spin) of $\frac{1}{2} .^{2}$ The electron appears to be structureless, while protons and neutrons are each composed of an additional set of fermions, the up and down quarks.

Analysis of radioactive decays, cosmic rays and high energy scattering experiments demonstrates the existence of other particles. The leptons, which include the electron, are apparently fundamental. The proton and neutron are part of a large spectrum of hadrons that is suggestive of a deeper pattern. Indeed these particles consist of various combinations of the different species of quarks, which appear to be structureless.

The quarks and leptons all have spin- $\frac{1}{2}$ and are organized into three generations:

$$
\begin{aligned}
& \left(\begin{array}{l}
u \\
d
\end{array}\right) \quad\left(\begin{array}{l}
c \\
s
\end{array}\right) \quad\left(\begin{array}{l}
t \\
b
\end{array}\right) \\
& \left(\begin{array}{c}
\nu_{e} \\
e
\end{array}\right)\left(\begin{array}{c}
\nu_{\mu} \\
\mu
\end{array}\right)\left(\begin{array}{c}
\nu_{\tau} \\
\tau
\end{array}\right)
\end{aligned}
$$

\footnotetext{
${ }^{1}$ It should be noted that gravity is not described by the Standard Model. However, this force is too weak to play a measurable role in the tests conducted so far.

${ }^{2} \mathrm{~A}$ fermion is in fact a particle with half-integer spin $\left(\frac{1}{2}, \frac{3}{2} \ldots\right)$. However proton, the neutron and all of the fundamental fermions observed in nature are spin $\frac{1}{2}$.
} 
In the Standard Model, these matter particles interact through the exchange of spin-1 bosons (a boson more generally has an integer spin: 0, 1, 2..). Thus the electromagnetic force is mediated by the massless photon. Quarks are bound together in hadrons (the most common being the proton and neutron) by gluons, which carry the strong force. The weak force is responsible for radioactive $\beta$-decay and is described by the exhange of the $W$ and $Z$ bosons. Unlike the photon and gluon, the $W$ and $Z$ are massive. This fact implies that the weak force has an extremely short range and explains its apparent weakness.

The nature of these bosons is predicted by the principle of local gauge invariance. Formally, this means that the Standard Model Lagrangian should be unchanged when the fields that define it are altered according to the rules of certain gauge transformations which may vary with space and time. The non-interacting fermion Lagrangian violates this principle, and new fields that couple to the fermions are required to preserve the symmetry. These additional fields are precisely the force carriers (gauge bosons) that are observed in nature.

The rules of these gauge transformations are defined by specific symmetry groups. The quantum description of electromagnetism (quantum electrodynamics or QED), for example, is characterized by invariance under changes in the complex phase of charged fermion fields. This corresponds to the $U(1)$ group: the set of $1 \times 1$ unitary matrices (those matrices whose inverse is equal to the transpose of their complex conjugates).

Quantum chromodynamics (QCD) describes the strong force in terms of an $S U(3)$ symmetry between the three quark colors that are the strong force analog of electric charge. Here, $S U(3)$ refers to the group of $3 \times 3$ unitary matrices that also have determinants equal to one. An abritrary member of the $N \times N$ generalization of this group $(S U(N))$ may be written as $e^{i \vec{\lambda} \cdot \vec{\theta}(x)}$ where $\vec{\lambda}$ is a vector of $N^{2}-1$ constant $N \times N$ matrices that are the generators of the group; and $\vec{\theta}(x)$ is a set of arbitrary real functions that define how the element varies in space and time.

It will be seen that because of the non-zero $W$ and $Z$ masses, no gauge invariant description of the weak interactions, considered in isolation, exists. Electroweak unification solves this problem by imposing a larger $(S U(2) \otimes U(1))$ symmetery that is spontaneously broken, leaving only a residual $U(1)$ symmetry that describes QED, ${ }^{3}$ but also giving masses to weak bosons and predicting the existence of a new spin-0 (scalar) particle, the Higgs boson. Thus the weak and electromagnetic forces are integrated into a single electroweak theory.

\footnotetext{
${ }^{3}$ This residual symmetry is a mixture of the original $S U(2)$ and $U(1)$ groups.
} 


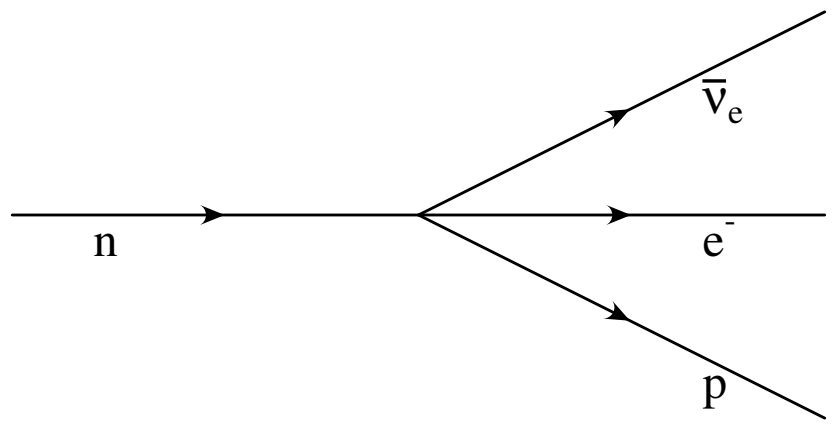

Figure 1.1: Feynman diagram for the Fermi theory of nuclear $\beta$-decay. The arrows indicate the progress of time.

\subsection{Electroweak Unification}

At low energies, nuclear $\beta$ decay is well described by the Fermi theory which models the process as a four particle, point interaction as shown in Figure 1.1. The matrix element is given by:

$$
\mathcal{M}=\frac{G_{F}}{\sqrt{2}}\left[\bar{n} \gamma^{\mu}\left(1-\gamma^{5}\right) p\right]\left[\bar{\nu}_{e} \gamma_{\mu}\left(1-\gamma^{5}\right) e\right]
$$

where $n, p, \nu_{e}$ and $e$ are the wavefunctions of the neutron, proton, electron and electron-neutrino [1]. The Fermi constant, $G_{F}$ is experimentally measured to be $1.166 \times 10^{-5} \mathrm{GeV}[2]$. The factors of $1-\gamma^{5}$ are present to describe the parity violating nature of $\beta$ decays suggested by Lee and Yang [3] and verified experimentally in the decay of ${ }^{60} \mathrm{Co}[4]$.

In the Standard Model, this is understood as an approximation of the exchange of a charged, massive boson, the $W$, as shown in Figure 1.2. At the low energies involved in $\beta$-decay, the internal $W$ propagator is far from the mass shell, thus reducing to the single vertex of the Fermi theory. The Fermi constant is thus seen as a combination of weak interaction strength $g$ and the $W$ mass, $M_{W}: G_{F} / \sqrt{2}=g^{2} / 8 M_{W}^{2}[5]$. Parity violation is enforced by coupling the $W$ only to left-handed fermions:

$$
\begin{aligned}
f_{L} & \equiv \frac{1}{2}\left(1-\gamma^{5}\right) f \\
f_{R} & \equiv \frac{1}{2}\left(1+\gamma^{5}\right) f
\end{aligned}
$$

This description of weak interactions may be unified with electromagnetism 


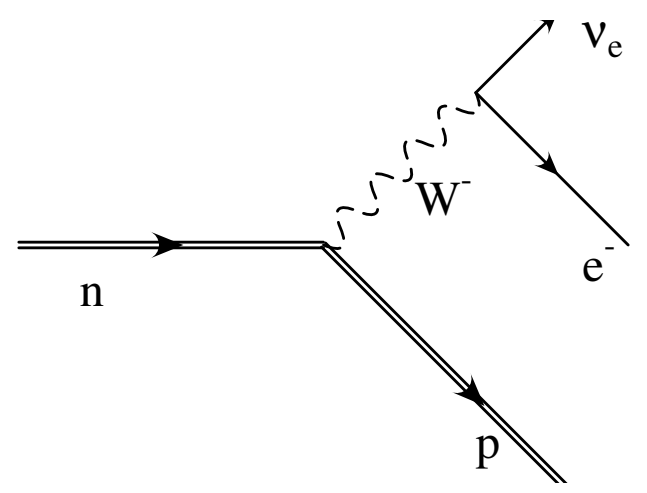

Figure 1.2: Feynman diagram for the Standard Model description of the decay of a neutron (here shown as composite particles). Since the energy in the initial state is much less than the $\mathrm{W}$ mass the internal propagator reduces to the effective vertex shown in Figure 1.1.

into a single theory invariant under $U(1)$ hypercharge gauge transformations acting on all fermions and $S U(2)$ isospin rotations acting on left-handed doublets [6]. Right-handed fermions are considered to be isospin singlets (i.e., they are unchanged by $S U(2)$ transformations and thus do not interact with the corresponding gauge fields). Therefore the particle content in the first generation of the fermion sector is:

$$
q_{L}=\left(\begin{array}{c}
u_{L} \\
d_{L}
\end{array}\right), u_{R}, d_{R}, l_{L}=\left(\begin{array}{c}
\nu_{L} \\
e_{L}
\end{array}\right), e_{R}
$$

For the purposes of this dissertation, there are no right-handed neutrinos. ${ }^{4}$ The second and third fermion generations replicate this structure. The righthanded singlets are invariant under $S U(2)$ transformations. The rule for lefthanded doublets is:

$$
f_{L} \rightarrow e^{i g \vec{\tau} \cdot \vec{\theta}(x)} f_{L}
$$

where $\vec{\tau}$ is the vector of isospin generators $\tau_{i=1,2,3}$ (identical to the familiar Pauli spin matrices); $\vec{\theta}(x)$ is a vector of arbitrary (real) scalar functions; and $g$ is the $S U(2)$ interaction strength. The $U(1)$ transformation rule is:

$$
f \rightarrow e^{i g^{\prime} \frac{Y}{2} \xi(x)} f
$$

\footnotetext{
${ }^{4}$ No attempt is made to discuss the problems of neutrino masses. At present, the Technicolor models explored herein are still immature in this area and cannot yet generate masses for the neutrino sector.
} 


\begin{tabular}{|c|cccc|}
\hline Fermion & $T$ & $T_{3}$ & $Y$ & $Q$ \\
\hline$\nu_{e, L}$ & $\frac{1}{2}$ & $\frac{1}{2}$ & -1 & 0 \\
$\mathrm{e}_{L}$ & $\frac{1}{2}$ & $-\frac{1}{2}$ & -1 & -1 \\
$\mathrm{e}_{R}$ & 0 & 0 & -2 & -1 \\
\hline $\mathrm{u}_{L}$ & $\frac{1}{2}$ & $\frac{1}{2}$ & $\frac{1}{3}$ & $\frac{2}{3}$ \\
$\mathrm{~d}_{L}$ & $\frac{1}{2}$ & $-\frac{1}{2}$ & $\frac{1}{3}$ & $-\frac{1}{3}$ \\
$\mathrm{u}_{R}$ & $\frac{1}{2}$ & 0 & $\frac{4}{3}$ & $\frac{2}{3}$ \\
$\mathrm{~d}_{R}$ & $\frac{1}{2}$ & 0 & $-\frac{2}{3}$ & $-\frac{1}{3}$ \\
\hline
\end{tabular}

Table 1.1: Quantum numbers for the first generation of quarks and leptons, which are replicated for the second and third generations. Shown are the total isospin $(T)$, its third component $\left(T_{3}\right)$, hypercharge $(Y)$ and electric charge $Q=T_{3}+Y / 2$. Note that the left and right handed components of the fields have differing quantum numbers.

Here the interaction strength is given by $g^{\prime}$ and $\xi(x)$ is another real function. The hypercharge, $Y$, is the conserved quantum number resulting from the $U(1)$ symmetry as the isospin components, $T_{i=1,2,3}$, are conserved because of the $S U(2)$ symmetry. Isospin and hypercharge are related to the conventional electric charge, $Q$, by

$$
Q=T_{3}+\frac{Y}{2}
$$

Table 1.1 gives the isospin, hypercharge and electric charge for the first generation of quarks and leptons.

Since $\theta$ and $\xi$ have non-zero derivatives, the kinetic part of the fermion Lagrangian, $\bar{f} \gamma^{\mu} \partial_{\mu} f$, is not invariant under $S U(2)$ or $U(1)$ transformations. The invariance can only be preserved by positing a new set of vector fields $B_{\mu}$ and $W_{\mu}^{i=1,2,3}$, transforming according to:

$$
\begin{aligned}
W_{\mu}^{i} & \rightarrow W_{\mu}^{i}+\partial_{\mu} \theta^{i}+g \epsilon^{i j k} W_{\mu}^{j} \theta^{k} \\
B_{\mu} & \rightarrow B_{\mu}+\partial_{\mu} \xi
\end{aligned}
$$

The symmetry is achieved by coupling the vector fields to the fermion fields. Thus the Lagrangian (restricted to the electron sector for simplicity) is:

$$
\begin{aligned}
\mathcal{L}= & -\frac{1}{4} B^{\mu \nu} B_{\mu \nu}-\frac{1}{4} W^{i, \mu \nu} W_{\mu \nu}^{i}+i \bar{e}_{R} \gamma^{\mu}\left(\partial_{\mu}+i g^{\prime} \frac{Y}{2} B_{\mu}\right) e_{R} \\
& +i \bar{l}_{L} \gamma^{\mu}\left(\partial_{\mu}+i g^{\prime} \frac{Y}{2} B_{\mu}+i g \tau^{i} W_{\mu}^{i}\right) l_{L}
\end{aligned}
$$


Here $Y$ is the hypercharge operator and $B_{\mu \nu}$ and $W_{\mu \nu}^{i}$ are the field strength tensors, giving the free Lagrangian for $B_{\mu}$ and $W_{\mu}^{i}$ :

$$
\begin{aligned}
B_{\mu \nu} & =\partial_{\mu} B_{\nu}-\partial_{\nu} B_{\mu} \\
W_{\mu \nu}^{i} & =\partial_{\mu} W_{\nu}^{i}-\partial_{\nu} W_{\mu}^{i}+g \epsilon^{i j k} W_{\mu}^{j} W_{\nu}^{k}
\end{aligned}
$$

Note the absence of any mass terms ( $m \bar{f} f$ for fermions, or $m^{2} B^{\mu} B_{\mu} / 2$ for a gauge field) in equation 1.9. Such terms are not, by themselves, gauge invariant. This issue is the motivation for this dissertation and will be addressed in detail later.

The Lagrangian may be rewritten in terms of the photon $\left(A_{\mu}\right)$ and charged $\mathrm{W}\left(W^{ \pm}\right)$fields, as well as an additional, neutral particle, the $Z\left(Z_{\mu}\right)[6]$ :

$$
\begin{aligned}
W_{\mu}^{ \pm} & =\frac{1}{\sqrt{2}}\left(W_{\mu}^{1} \mp i W_{\mu}^{2}\right) \\
A_{\mu} & =\cos \theta_{W} B_{\mu}+\sin \theta_{W} W_{\mu}^{3} \\
Z_{\mu} & =-\sin \theta_{W} B_{\mu}+\cos \theta_{W} W_{\mu}^{3}
\end{aligned}
$$

where the weak mixing angle, $\theta_{W}$, is defined by $\cos \theta_{W}=g / \sqrt{g^{2}+g^{2}}$ and $\sin \theta_{W}=g^{\prime} / \sqrt{g^{2}+g^{\prime 2}}$. Examining the interaction terms, equation 1.6 is recovered, and the electromagnetic interaction strength is seen to be $g g^{\prime} / \sqrt{g^{2}+g^{2}}=$ $g \sin \theta_{W}=g^{\prime} \cos \theta_{W}$.

\subsection{The Higgs Mechanism}

Electroweak unification thus formulated is built on the principle of gauge invariance (as is QCD), which provides a natural method for deriving the dynamics of the interactions. Gauge invariance also ensures that the theory may be renormalized [5]. But if the transformation rules in equations 1.7 and 1.8 are applied to the $\mathrm{W}$ and $\mathrm{Z}$ fields, it is seen that the required mass terms (e.g., $M_{Z}^{2} Z^{\mu} Z_{\mu} / 2$ for the $Z$ ) are not invariant under either $U(1)$ or $S U(2)$ transformations (and likewise for the $W$ ). Furthermore the electron mass term (for example) is proportional to $\bar{e} e=\left(\bar{e}_{L}+\bar{e}_{R}\right)\left(e_{L}+e_{R}\right)$. The terms $\bar{e}_{R} e_{R}$ and $\bar{e}_{L} e_{L}$, vanish, leaving only the cross-terms, but these also are not gauge invariant.

The simplest solution to these difficulties is Higgs mechanism [6], [7] which posits a scalar (i.e., spin-0) isospin doublet $\phi$ with $Y=+1$ :

$$
\phi \equiv\left(\begin{array}{c}
\phi^{+} \\
\phi^{0}
\end{array}\right)
$$


The Lagrangian for $\phi$ connects it to the all of the other fields in electroweak theory (except, when they are assumed to be massless, neutrinos):

$$
\begin{aligned}
\mathcal{L}_{\text {Higgs }} & =\left|\partial_{\mu} \phi+i g W_{\mu}^{i} \tau^{i} \phi+i \frac{1}{2} g^{\prime} B_{\mu} \phi\right|^{2} \\
& -G_{e}\left(\bar{l}_{L} \phi e_{R}+\bar{e}_{R} \phi^{\dagger} l_{L}\right)-\mu^{2} \phi^{\dagger} \phi-\lambda\left(\phi^{\dagger} \phi\right)^{2}
\end{aligned}
$$

where $G_{e}, \mu$ and $\lambda$ are free parameters of the theory, whose meaning will emerge shortly. The potential energy of the field is thus $V(\phi)=\mu^{2} \phi^{\dagger} \phi+\lambda\left(\phi^{\dagger} \phi\right)^{2}$. If $\mu^{2}<0<\lambda$ the ground (vacuum) states are degenerate and spontaneously break the $S U(2) \otimes U(1)$ symmetry of the theory: a particular ground state picks out a direction in $S U(2)$ space, violating the symmetry, while the set of all vacuum states preserves it. These states have an expectation value, $<|\phi|>_{0}=v / \sqrt{2}=\sqrt{-\mu^{2} / 2 \lambda}$, and it is possible to arbitrarily choose a ground state:

$$
<\phi>_{0}=\left(\begin{array}{c}
0 \\
\frac{v}{\sqrt{2}}
\end{array}\right)
$$

Excitations of $\phi$ may be characterized by expanding it around the vacuum:

$$
\phi=\exp \left(i \frac{\vec{\tau} \cdot \vec{\zeta}}{2 v}\right)\left(\begin{array}{c}
0 \\
\frac{v+\eta}{\sqrt{2}}
\end{array}\right)
$$

in terms of the four real scalar fields $\eta$ and $\zeta_{1,2,3}$. Since the exponential factor is just an $S U(2)$ rotation matrix, it may be transformed away. The Lagrangian may be re-expressed in terms of $\eta$ and the physical vector bosons, giving mass terms for the fermions, the $W$ and the $Z$, while the photon remains massless. The $\zeta$ fields are the massless spin- 0 Nambu-Goldstone bosons predicted in the spontaneous breakdown of any continuous symmetry [6] and are absorbed (eaten) into the transformed vector fields and thus describe the longitudinally polarized states of the $W$ and $Z$ (forbidden for massless bosons). This combination of the Nambu-Goldstone bosons and otherwise massless gauge fields is called the Higgs mechanism. In this context $\eta$ is seen as the field for a new neutral, spin-0, particle, the Higgs boson. The complete electroweak theory predicts:

$$
M_{W}=\frac{g v}{2}=M_{Z} \cos \theta_{W}
$$

Direct measurements of the weak mixing angle and the $W$ and $Z$ masses are in excellent agreeement with this prediction. The vacuum expecation value, $v$, has a simple relationship to the Fermi constant:

$$
v=\left(G_{F} \sqrt{2}\right)^{-1 / 2} \approx 246 \mathrm{GeV}
$$


and the parameter $G_{e}$ is now understood to be the electron-Higgs interaction strength, and is proportional to the electron mass: $m_{e}=G_{e} v / \sqrt{2}$.

The Higgs mass, $M_{H}=|\mu| \sqrt{2}$, remains unpredicted, although measurements of the $\mathrm{W}$ and top mass constrain it to be less than $207 \mathrm{GeV} / \mathrm{c}^{2}$ at the 95\% confidence level [8]. Direct searches for the Higgs boson at LEP have been unsuccessful, resulting in a lower bound of $\sim 114 \mathrm{GeV} / c^{2}[9]$.

\subsection{An Alternative Solution - Technicolor}

Despite the striking successes of electroweak theory, the use of an elementary scalar field to generate the Standard Model masses is problematic [10]. The issue of naturalness arises from the observation that radiative corrections to the Higgs mass are quadratically divergent (i.e.when corrections due to particle loops in the Higgs propagator are calculated, they are found to be proportional to the square of the scale $\Lambda$ at which the loop momentum integral is cut off by the renormalization procedure). Furthermore, if electroweak theory is unified with QCD at some very high scale (typically taken to be $10^{16} \mathrm{GeV}$ ) then the Higgs mass should have a similar value. This is called the hierarchy problem. Both of these issues can be resolved only by finely tuned cancellations, precise to $\sim 40$ decimal places. Finally, there is the triviality problem; due to the fact that the renormalization of the Higgs self-coupling, $\lambda$, drives it to zero at scales of order $1 \mathrm{TeV}$. The issue of triviality implies that the Standard Model is, at best, an approximate theory and the essential physics of electroweak symmetry breaking must change at higher energies.

To address these isssues, it is instructive to note that the strong force can provide a small mass to the $W$ and $Z$ bosons. Consider, the Standard Model without the Higgs field. When the up and down quarks are held to be massless, the QCD Lagrangian obeys a global, $S U(2)_{L} \otimes S U(2)_{R}$ (chiral) symmetry:

$$
\begin{aligned}
q_{L} & =\left(\begin{array}{c}
u_{L} \\
d_{L}
\end{array}\right) \rightarrow U_{L} q_{L} \\
q_{R} & =\left(\begin{array}{l}
u_{R} \\
d_{R}
\end{array}\right) \rightarrow U_{R} q_{R}
\end{aligned}
$$

where the $U_{L, R}$ are constant $S U(2)$ rotation matrices. At low energies, the renormalization of QCD causes the interaction strength, $g_{S}$, to become large, forming the bound state hadrons [11]. The effective Lagrangian of the bound states contains the spin-0 pions with a potential similar to the Higgs potential in equation 1.16 and the chiral symmetry is spontaneously broken. Since the quarks also couple to the electroweak bosons the $S U(2) \otimes U(1)$ symmetry 
is broken as well. The pions become the requisite Nambu-Goldstone bosons (NGB's) and are absorbed into the $W$ and $Z$ fields via the Higgs mechanism. The $W$ mass is thus:

$$
M_{W}=\frac{g f_{\pi} \sqrt{3}}{2} \approx 50 \mathrm{MeV} / c^{2}
$$

Here the pion decay constant $f_{\pi} \approx 93 \mathrm{MeV}$ plays the same role as the Higgs vacuum expecation value. Since the up and down quarks have non-zero masses the chiral symmetry of the theory is only approximate. As a result the pions are not eaten by the $W$ and $Z$ but are separate physical states with observable masses. Notably, this mechanism also respects the relationship between the weak parameters predicted by the Standard Model (see equation 1.19).

Technicolor is a theory, modeled on QCD, that breaks electroweak symmetry in the dynamically driven manner described in the previous paragraph [11]. The simplest version introduces a new $S U\left(N_{T C}\right)$ gauge interaction acting on a new set of fundamental fermions, the techniquarks, with $N_{T C}$ technicolors. The techniquarks also form left-handed weak-isospin doublets with hypercharge 0 and right handed singlets with hypercharge $\frac{1}{2}$ :

$$
Q_{L}=\left(\begin{array}{c}
U_{L} \\
D_{L}
\end{array}\right), U_{R}, D_{R}
$$

Technicolor also respects an exact version of the chiral symmetry between quark flavors.

The renormalization of the theory causes the interaction strength, $g_{T C}$, to become large at energies below some scale, $\Lambda_{T C}$. To generate the observed weak boson masses, $\Lambda_{T C}$ should be several hundred GeV. The techniquarks form bound states whose vacuum breaks both the electroweak and chiral symmetries:

$$
<\bar{U} U+\bar{D} D>\neq 0
$$

The lightest composite particles, the technipions, are the Nambu-Goldstone bosons predicted by the symmetry breaking, and are eaten to form the longitudinally polarized $W$ and $Z$ bosons. The $W$ mass can be written in terms of the technipion decay constant $F_{T}$ in analogy to equation 1.23 .

$$
M_{W}=\frac{g F_{T} \sqrt{N_{D}}}{2}=\frac{g v}{2}
$$

Therefore, $F_{T} \sqrt{N_{D}}=v=246 \mathrm{GeV}$. The factor of $\sqrt{N_{D}}$ allows for an arbitrary number of Technicolor electroweak doublets, whereas the factor of $\sqrt{3}$ from 
equation 1.23 arises from the fact that there are three generations of quarks. Additional bound states, such as the Technicolor equivalents of the $\rho$ and $\omega$ mesons, are also predicted. Since the Technicolor, like QCD, is an asymptotically free theory (i.e., the interaction strength becomes small at high energies), the bound state approach breaks down at energies above $\Lambda_{T C}$. At these scales, any spin-0 particles are treated in terms of their constituent fermions which are not susceptible to the issues of naturalness, hierarchy and triviality discussed earlier.

\subsubsection{Extensions to Technicolor}

This model of a dynamically driven breakdown of electroweak symmetry is certainly attractive, but the minimal model described above is insufficient [11]. It provides no mechanism for generating fermion masses. Furthermore, when $N_{D}>1$ the intial chiral symmetry group that is broken is enlarged. This induces more approximately massless pseudo Nambu-Goldstone bosons (PNGB's) that should be readily observable. Finally, since minimal Technicolor also conserves techniquark number, the technibaryons should be stable, a prediction inconsistent with cosmological data.

Extended Technicolor (ETC) addresses all of these issues by embedding the QCD $S U(3)$ symmetery group and the Technicolor group $S U\left(N_{T C}\right)$ in a single master gauge symmetry, $G_{E T C}$, that acts on leptons as well. This group is dynamically broken at some scale $\Lambda_{E T C}$ to the Technicolor and QCD symmetries (and possibly others), which undergo their own symmetry breaking at lower energy scales $\Lambda_{T C}$ and $\Lambda_{Q C D}$. The coupling between the Standard Model fermions and the ETC gauge bosons provides a mechanism for technibaryon decay and generates masses for the conventional fermions. Unfortunately, this mechanism does not, in general, address the issue of light scalar fields (the PNGB's). Extended Technicolor is also inconsistent with precision electrwoeak measurements (e.g., a $\sim 5 \%$ enchancement of $\Gamma(Z \rightarrow b \bar{b}) / \Gamma(Z \rightarrow$ hadrons $)$ ). Finally, flavor changing neutral current (FCNC) interactions such as $e e \rightarrow e \mu$ are predicted, but not observed in the laboratory. These may be suppressed, but this generally requires fermion masses of less than $100 \mathrm{MeV} / c^{2}$.

It has been observed that, if $g_{T C}$ is approximately constant between $\Lambda_{T C}$ and $\Lambda_{E T C}$, the FCNC terms may be suppressed while allowing fermion masses consistent with the charm and bottom quarks $\left(m_{q} \sim 1 \mathrm{GeV} / c^{2}\right)$ [11]. Furthermore, masses of $\mathcal{O}\left(N_{T C} \times \Lambda_{T C}\right)$ are generated for the PNGB's. While such behavior is unlike QCD, it may emerge from appropriate choices for the defining gauge group (i.e., $N_{T C}$ ) and the number of particpating fermions. Thus, the defects mentioned in the previous paragraph become constraints on the 
particular structure of realistic ETC models. Such instances are referred to as Walking Technicolor (WTC) theories.

However, even WTC models are only marginally consistent with the bottom quark mass and are wholly incompatible with the top quark mass of 172.5 $\mathrm{GeV} / c^{2}[12]$. Although the Standard Model allows for the possibilty, the fact that $m_{t}$ is so much larger than the other quark masses is puzzling and has inspired new theories, such as Topcolor, that propose a separate mechanism for the top mass [11].

In the simplest fomulation the third generation of quarks ( $t$ and $b$ ) participate in a $S U(3)$ interaction (Topcolor) separate from and stronger than the first two generations. This $S U(3)_{1,2} \otimes S U(3)_{3}$ symmetry is dynamically broken to the conventional QCD. In the process a scalar $t \bar{t}$ condensate is formed that plays the role of the Higgs, but also gives an especially large mass to the top quark, due to its composition.

To prevent Topcolor scale $b \bar{b}$ bound-states from forming, the weak hypercharge symmetry is likewise embedded in a broken $U(1)_{1,2} \otimes U(1)_{3}$ group. The gauge fields mix to produce the hypercharge boson and a new massive particle, the $Z^{\prime}$, just as the photon and $Z$ are orthognal mixtures of the massless hypercharge and third isospin gauge bosons (see equations 1.13 and 1.14). For an appropriate choice of the mixing angle, the relative sign of the term that would give rise to the $b \bar{b}$ composite particle becomes inverted and the corresponding force becomes repulsive for bottom quarks while still attractive for top quarks. Since no heavy $b \bar{b}$ condensate is formed, $m_{b}$ remains small.

In Topcolor-assisted Technicolor (TC2), the walking (i.e., slow evolution) of the WTC interaction strength breaks the Topcolor symmetries, removing fine-tuning issues that arise in Topcolor models. All the quarks (including top) are thus seen to acquire masses of $\sim 1 \mathrm{GeV} / c^{2}$ or less from WTC. However, $m_{t}$ recieves an additional, very large, contribution from the $t \bar{t}$ condensate.

The picture of electroweak symmetry breaking presented here envisions some new, strong gauge interaction, Extended Technicolor, that acts on all weakly interacting particles, including a new set of techniquarks. The master ETC symmetry is broken by the dynamics of the theory, possibly in multiple stages. This generates small masses for the fermions. The residual symmetries of Technicolor, Topcolor and electroweak interactions are broken as the Technicolor coupling becomes strong and masses are generated for the weak bosons. The Topcolor symmetry breaking produces a $t \bar{t}$ bound state which gives a large additional mass only to the top quark. To remain consistent with existing precision electroweak measurements and FCNC limits, the structure of the Extended Technicolor sector must be such that $g_{T C}$ evolves slowly when renormalized. Finally, a rich spectrum of technihadrons is predicted. 


\subsubsection{Low Scale Technicolor Phenomenology}

It is this array of Technicolor bound states that is subject to direct experimental verification. The details depend on the particular choices made for the structure of the model (e.g., the Extended Technicolor gauge group and interaction strength). This dissertation tests the Technicolor Straw Man (TCSM) model [13], which predicts an assortment of low scale technimesons (i.e., particles with electroweak scale masses). More massive states, including technibaryons, are also predicted but this discussion will be limited to the lighter particles.

It should be emphasized that the parameters introduced herein are not fundamental inputs to the theory. Rather, they emerge from the dynamics of Technicolor just as an array of similar parameters arise from QCD. The only fundamental inputs are the nature of the TC2 gauge interactions (i.e., the coupling strengths and symmetry groups) and the structure of the technifermion sector (the Standard Model charges and the number of technifermions). The calculation of the emergent parameters may be difficult, as in QCD. In such cases, the wealth of experimental tests of and theoretical calculations with strong interactions provides insight as to the values that these parameters take on by scaling the corresponding parameters of QCD.

The lightest bound states of the theory are the color-singlet technipions, which are grouped into an isospin vector, $\Pi_{T}^{ \pm, 0}$ and an isosinglet $\Pi^{0^{\prime}}$. The isotriplet states, however, do not correspond to physical particles. Rather, they are assumed to be superpositions of the NGB's that are eaten to form the longitudinal states of the weak bosons, $W_{L}^{ \pm}$and $Z_{L}^{0}$, and a set of technipion mass eigenstates, $\pi_{T}^{ \pm, 0}$ :

$$
\left|\Pi_{T}>=\sin \chi\right| W_{L}>+\cos \chi \mid \pi_{T}>
$$

where the assumed mixing angle, $\chi$, is expected to be small. The isovector technipions couple to Standard Model fermions like elementary Higgs bosons, with some additional model dependent factors:

$$
\Gamma\left(\pi_{T}^{ \pm, 0} \rightarrow \bar{f}^{\prime} f\right)=\frac{1}{16 \pi F_{T}^{2}} N_{f} p_{f} C_{1 f}^{2}\left(m_{f}+m_{f^{\prime}}\right)^{2}
$$

where $N_{f}$ is the number of (QCD) colors of $f ; p_{f}$ is the fermion momentum in the technirho rest frame; the ETC parameter, $C_{1 f}$, is typically of order one. When the final state includes a top quark, it is roughly $m_{b} / m_{t}$ and such decays are suppressed.

In addition to the pseudoscalar technipions, a set of spin-1 technimesons are predicted. In analogy to QCD, these include an isovector of particles, $\rho_{T}^{ \pm, 0}$ 
and a neutral isospin singlet, $\omega_{T}$. They are produced through a process known as vector meson dominance, in which the technimeson mixes with electroweak gauge bosons due to terms in the effective Lagrangian that are bilinear in the gauge and technimeson field strength tensors (e.g., $F_{\mu \nu}^{Z} F_{\rho}^{\mu \nu}$ in the case of the $Z$ and the neutral $\left.\rho_{T}\right)[11]$.

The $\rho_{T}$ interactions with technipions, $\Pi_{T}^{ \pm, 0}$, are dominated by Technicolor. The electroweak couplings may therefore be taken to vanish and the resulting decay rates are:

$$
\Gamma\left(\rho_{T} \rightarrow \pi_{A} \pi_{B}\right)=\frac{2 \alpha_{\rho_{T}} \mathcal{C}_{A B}^{2}}{3} \frac{p^{3}}{\hat{s}}
$$

where $p$ is the technipion momentum in the $\rho_{T}$ rest frame and $\hat{s}$ is the (possibly off-shell) mass of the the technirho; the coupling constant $\alpha_{\rho_{T}}$ is, by analogy with QCD, $2.91 \times\left(3 / N_{T C}\right)$. The factor $\mathcal{C}_{A B}$ depends on the specific final state, which because of the mixing from equation 1.27, may include longitudinally polarized gauge bosons:

$$
\mathcal{C}_{A B}= \begin{cases}\sin ^{2} \chi & \text { for } \rho_{T}^{0} \rightarrow W_{L}^{ \pm} W_{L}^{\mp} \text { or } \rho_{T}^{ \pm} \rightarrow W_{L}^{ \pm} Z_{L} \\ \sin \chi \cos \chi & \text { for } \rho_{T}^{0} \rightarrow W_{L}^{ \pm} \pi_{T}^{\mp} \text { or } \rho_{T}^{ \pm} \rightarrow W_{L}^{ \pm} \pi_{T}^{0}, Z_{L} \pi_{T}^{ \pm} \\ \cos ^{2} \chi & \text { for } \rho_{T}^{0} \rightarrow \pi_{T}^{ \pm} \pi_{T}^{\mp} \text { or } \rho_{T}^{ \pm} \rightarrow \pi_{T}^{ \pm} \pi_{T}^{0}\end{cases}
$$

The corresponding rate for the process $\omega_{T} \rightarrow W_{L}^{ \pm} \pi_{T}^{\mp}$ is $\left|\epsilon_{\rho \omega}\right|^{2} \Gamma\left(\rho^{0} \rightarrow\right.$ $W_{L}^{ \pm} \pi_{T}^{\mp}$, where $\epsilon_{\rho \omega} \approx 0.05$ is a mixing amplitude from QCD. Thus, $\omega_{T}$ decays to longitudinally polarzied states are supressed. The preferred channels are dominated by $\bar{f} f$ states as well as those including transversely polarized gauge bosons $\left(e . g \cdot \gamma \pi_{T}^{0}, W_{\perp}^{ \pm} \pi_{T}^{\mp} \ldots\right)$.

The rate for a vector technimeson state, $V_{T}=\left\{\rho_{T}, \omega_{T}\right\}$, to decay to a technipion and some electroweak boson, $G$, with mass, $M_{G}$, and transverse polarization is:

$$
\Gamma\left(V_{T} \rightarrow G \pi_{T}\right)=\frac{\alpha_{E M} V_{V_{T} G \pi_{T}}^{2} p^{3}}{3 M_{V}^{2}}+\frac{\alpha_{E M} A_{V_{T} G \pi_{T}}^{2} p\left(3 M_{G}^{2}+2 p^{2}\right)}{6 M_{A}^{2}}
$$

where $p$ is the momentum of $G$ relative to $V_{T}$; the scale parameters $M_{V}$ and $M_{A}$ are, invoking QCD once more, expected to be several hundred $\mathrm{GeV}$; the vector and axial coefficients, $V_{V_{T} G \pi_{T}}$ and $A_{V_{T} G \pi_{T}}$ depend on the specific model parameters and the final state. Sample values, as well as a total decay rate are shown in Table 1.2.

\subsection{Previous Searches}

There have been several searches for the production of Technicolor particles in recent years, both at the Tevatron and at LEP. Before discussing the search 


\begin{tabular}{lccc}
\hline \hline Process & $V_{V_{T} G \pi_{T}}$ & $A_{V_{T} G \pi_{T}}$ & $\Gamma\left(V_{T} \rightarrow G \pi_{T}\right)$ \\
\hline \hline$\omega_{T} \rightarrow \gamma \pi_{T}^{0}$ & $c_{\chi}$ & 0 & $0.115 c_{\chi}^{2}$ \\
$\omega_{T} \rightarrow \gamma \pi_{T}^{0^{\prime}}$ & $\left(Q_{U}+Q_{D}\right) c_{\chi^{\prime}}$ & 0 & $0.320 c_{\chi^{\prime}}^{2}$ \\
$\omega_{T} \rightarrow Z \pi_{T}^{0}$ & $c_{\chi} \cot 2 \theta_{W}$ & 0 & $2.9 \times 10^{-3} c_{\chi}^{2}$ \\
$\omega_{T} \rightarrow Z \pi_{T}^{0^{\prime}}$ & $-\left(Q_{U}+Q_{D}\right) c_{\chi^{\prime}} \tan 2 \theta_{W}$ & 0 & $5.9 \times 10^{-3} c_{\chi^{\prime}}^{2}$ \\
$\omega_{T} \rightarrow W^{ \pm} \pi_{T}^{\mp}$ & $c_{\chi} /\left(2 \sin \theta_{W}\right)$ & 0 & $2.4 \times 10^{-2} c_{\chi}^{2}$ \\
\hline$\rho_{T}^{0} \rightarrow \gamma \pi_{T}^{0}$ & $\left(Q_{U}+Q_{D}\right) c_{\chi}$ & 0 & $0.320 c_{\chi}^{2}$ \\
$\rho_{T}^{0} \rightarrow \gamma \pi_{T}^{0^{\prime}}$ & $c_{\chi^{\prime}}$ & 0 & $0.115 c_{\chi^{\prime}}^{2}$ \\
$\rho_{T}^{0} \rightarrow Z \pi_{T}^{0}$ & $-\left(Q_{U}+Q_{D}\right) c_{\chi} \tan \theta_{W}$ & 0 & $5.9 \times 10^{-3} c_{\chi}^{2}$ \\
$\rho_{T}^{0} \rightarrow Z \pi_{T}^{0^{\prime}}$ & $c_{\chi^{\prime}} \cot 2 \theta_{W}$ & 0 & $2.9 \times 10^{-3} c_{\chi^{\prime}}^{2}$ \\
$\rho_{T}^{0} \rightarrow W^{ \pm} \pi_{T}^{\mp}$ & 0 & $\pm c_{\chi} /\left(2 \sin \theta_{W}\right)$ & $2.4 \times 10^{-2} c_{\chi}^{2}$ \\
\hline$\rho_{T}^{ \pm} \rightarrow \gamma \pi_{T}^{ \pm}$ & $\left(Q_{U}+Q_{D}\right) c_{\chi}$ & 0 & $0.320 c_{\chi}^{2}$ \\
$\rho_{T}^{ \pm} \rightarrow Z \pi_{T}^{ \pm}$ & $-\left(Q_{U}+Q_{D}\right) c_{\chi} \tan \theta_{W}$ & $\left.\pm c_{\chi} / \sin 2 \theta_{W}\right)$ & $0.153 c_{\chi}^{2}$ \\
$\rho_{T}^{ \pm} \rightarrow W^{ \pm} \pi_{T}^{0}$ & 0 & $\mp c_{\chi} /\left(2 \sin \theta_{W}\right)$ & $0.143 c_{\chi}^{2}$ \\
$\rho_{T}^{ \pm} \rightarrow W^{ \pm} \pi_{T}^{0^{\prime}}$ & $c_{\chi}^{\prime} /\left(2 \sin \theta_{W}\right)$ & 0 & $2.4 \times 10^{-2} c_{\chi^{\prime}}^{2}$ \\
\hline \hline
\end{tabular}

Table 1.2: Coefficients and total decay rate (in $\mathrm{GeV}$ ) for $V_{T} \rightarrow G \pi_{T}$. For the purposes of this calculation, $M_{V_{T}}=210 \mathrm{GeV} / c^{2}, M_{\pi_{T}}=110 \mathrm{GeV} / c^{2}$, $M_{V}=M_{A}=100 \mathrm{GeV}$; the technifermion charges are chosen such that $\left(Q_{U}+\right.$ $\left.Q_{D}\right)=\frac{5}{3} ; ; c_{\chi}=\cos \chi$; an additional angle $\chi^{\prime}$ mixes the isosinglet $\Pi^{0^{\prime}}$ with bound states from additional technifermion doublets $c_{\chi^{\prime}}=\cos \chi^{\prime}[13]$. 
described herein, it is useful to survey the results of these studies. However, it should be noted that subsequent theoretical developments have made the interpretation of these analyses non-trivial.

The CDF collaboration has considered the production channels [14]:

$$
\begin{aligned}
& p \bar{p} \rightarrow \rho_{T} \rightarrow W \pi_{T} \rightarrow l \nu b j \\
& p \bar{p} \rightarrow \rho_{T} \rightarrow W \pi_{T} \rightarrow j j b j \\
& p \bar{p} \rightarrow \rho_{T} \rightarrow \pi_{T} \pi_{T} \rightarrow b j b j
\end{aligned}
$$

for $l=e, \mu$. The lepton plus jets analysis used a luminosity of $109 \mathrm{pb}^{-1}$ and demanded an isolated high $p_{T}$ electron or muon, two jets and a significant amount of missing transverse energy. To reduce the contribution of backgrounds without a $b$-jet, at least one of the jets was required to contain a displaced vertex, indicating the decay of a $B$ hadron ( $b$-tagged). Since heavy particles tend to be produced at rest, additional selection criteria were applied to the aziumuthal opening angle between the jets and their total transverse momentum. Finally, mass window requirements were imposed and a counting experiment was done. This allowed the exclusion of a region in the $M_{\pi}-M_{\rho}$ plane, shown in Figure 1.3.

The four-jets analysis used a data set of $91 \mathrm{pb}^{-1}$ and considers only the four highest energy jets in the event. At least two of the jets were required to have a secondary vertex. To reduce gluon splitting to $b \bar{b}$ backgrounds an azimuthal opening angle is imposed on the two tagged jets. The invariant mass distribution of the tagged jet system was added to the corresponding distribution from the lepton plus jets analysis and the result was compared to the expected background shape. Cross-section limits were set, but the exclusion region was not extended.

In a separate analysis using an $85 \mathrm{pb}^{-1}$ data set, CDF searched for $\omega_{T} \rightarrow$ $\gamma \pi_{T} \rightarrow \gamma b \bar{b}$ [15]. A photon and two jets were required, at least one of which should be $b$-tagged. No excess over expectations from the Standard Model and instrumental backgrounds was observed and a region of $M_{\omega}-M_{\pi}$ space, shown in Figure 1.4 was excluded.

The D $\varnothing$ collaboration has also conducted a search for $p \bar{p} \rightarrow \rho_{T} / \omega_{T} \rightarrow e^{+} e^{-}$ production with a luminosity of $125 \mathrm{pb}^{-1}$ [16]. The dielectron invariant mass sprectrum was examined for the presence of any resonances. Since the data were consistent with $Z / \gamma \rightarrow e^{+} e^{-}$production, a lower limit of approximately $200 \mathrm{GeV} / c^{2}$ (depending on the choice of model parameters) was set on the technirho and techniomega masses, as shown in Figure 1.5. However, this search assumed that the decay $\rho_{T} / \omega_{T} \rightarrow W \pi_{T}$ is kinematically forbidden, a region of parameter space orthogonal to one considered here. 


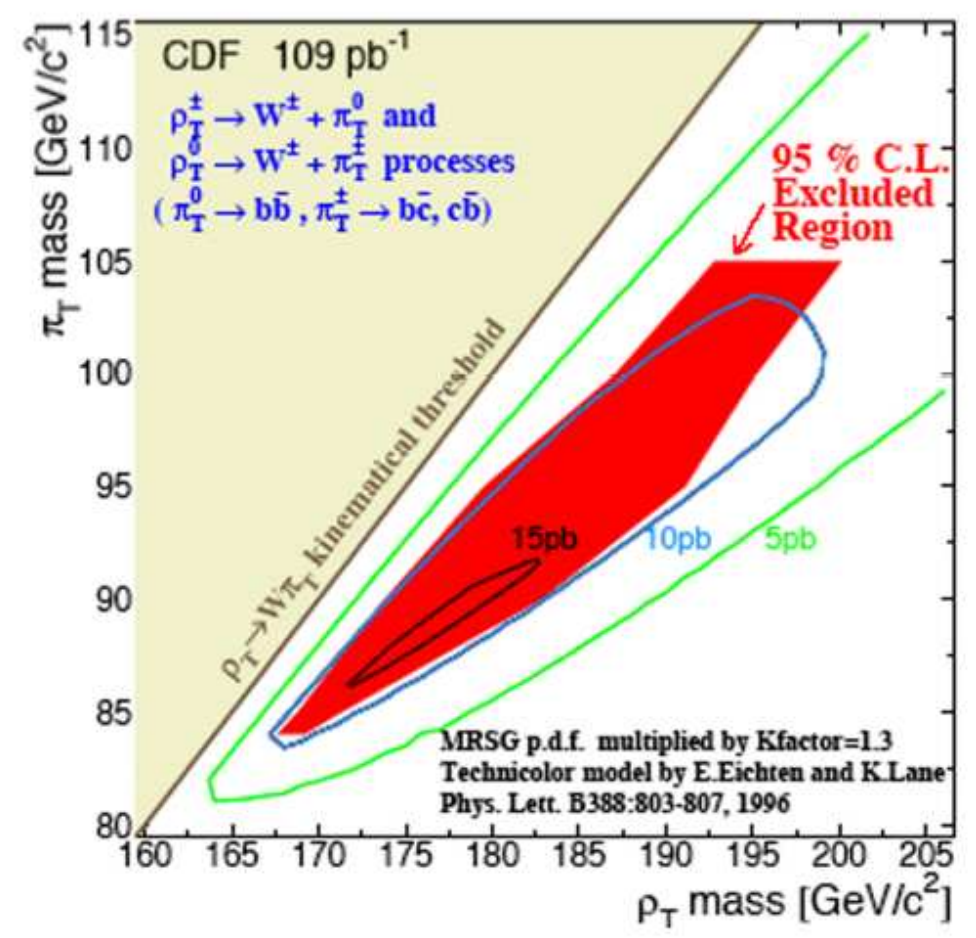

Figure 1.3: 95\% confidence level mass and cross section limits from the lepton plus jets $\rho_{T} \rightarrow W \pi_{T}$ search at the CDF experiment [14].

The DELPHI collaboration has examined $e^{+} e^{-}$collisions for evidence of Technicolor with an integrated luminosity of $452 \mathrm{pb}^{-1}$ in a variety of channels [17]. They observed good agreement with Standard Model expectations and exclude technipion masses less than $\sim 80 \mathrm{GeV} / c^{2}$ at the $95 \%$ confidence level. Further excluded is the region $90 \mathrm{GeV} / c^{2}<M_{\rho}<206.7 \mathrm{GeV} / c^{2}$.

Some care should be excercised in the interpretation of the these previous analyses. Revisions have been made to the TCSM since their publication [13]. The model used for the searches considered at Tevatron did not consider the decay of technimesons to transversely polarized gauge bosons. When these are included the probed cross-sections are suppressed by as much as a factor of two [18]. The DELPHI limit assumed an inappropriate narrow $\rho_{T}$ resonance approximation. Furthermore, the interference between Technicolor and Standard Model production amplitudes were not correctly modeled [18], [19]. 


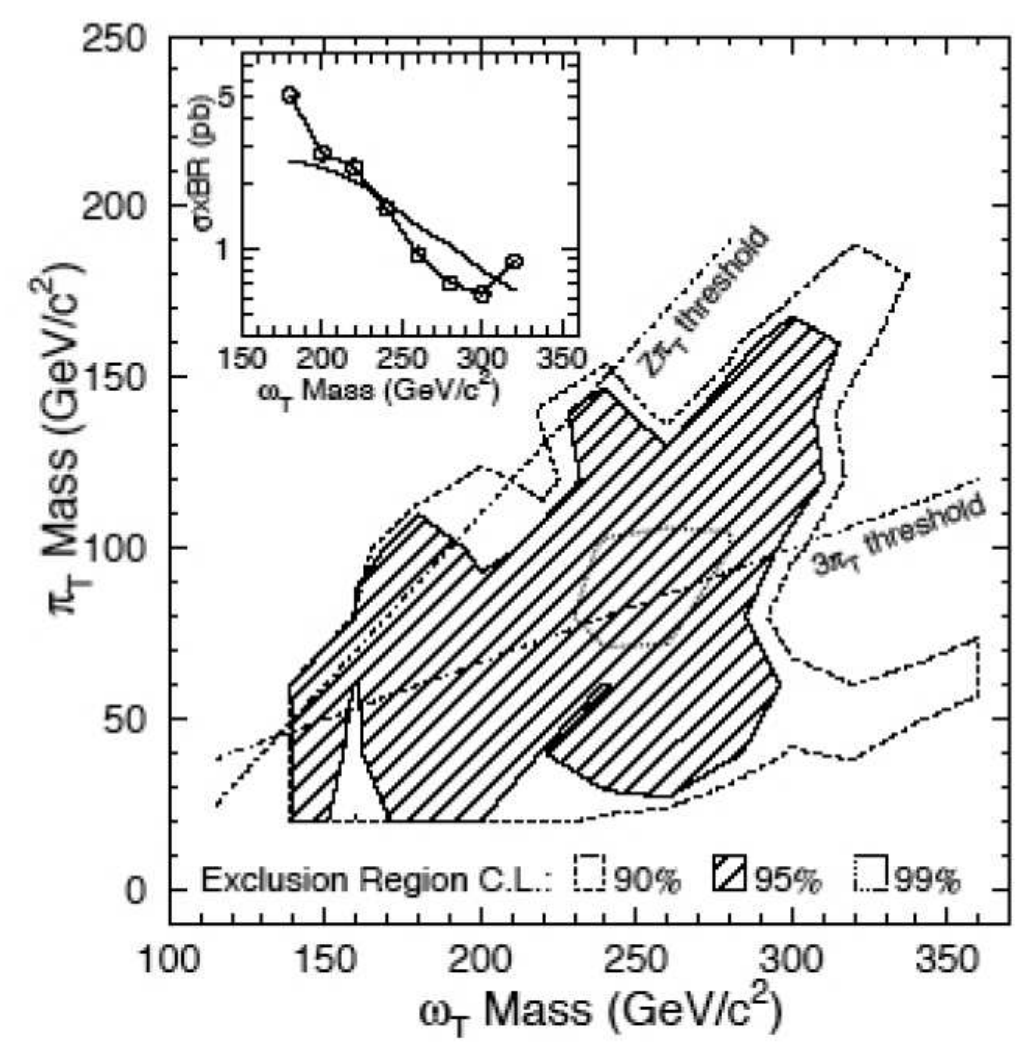

Figure 1.4: Exclusion regions from the CDF search for $\omega_{T} \rightarrow \gamma \pi_{T} \rightarrow \gamma b \bar{b}$ production [15].

\subsection{Searching for Technicolor at DØ Run II}

This dissertation presents the results of a search for the production of Technicolor particles, using a $300 \mathrm{pb}^{-1}$ data set, recorded by the the $\mathrm{D} \varnothing$ detector during Run II of the Tevatron $p \bar{p}$ collider. Specifically, the data were examined for evidence of $p \bar{p} \rightarrow \rho_{T}^{ \pm} \rightarrow W^{ \pm} \pi_{T}^{0} \rightarrow \mu \nu b \bar{b}$ and $p \bar{p} \rightarrow \rho_{T}^{0} \rightarrow W^{ \pm} \pi_{T}^{\mp} \rightarrow \mu \nu b \bar{c}$. Feynman diagrams for these process are shown in Figure 1.6. A similar search has been conducted concurrently for the case $W \rightarrow e \nu$ [18]. It will be discussed in the concluding chapter.

Twenty hypotheses for the masses of the $\rho_{T}$ and $\pi_{T}$ technimesons were considered. Discussion of the mass points used and the determination of the cross-sections times branching ratios is deferred until section 6.1. The parameters of the low energy approximate theory were the model defaults suggested 


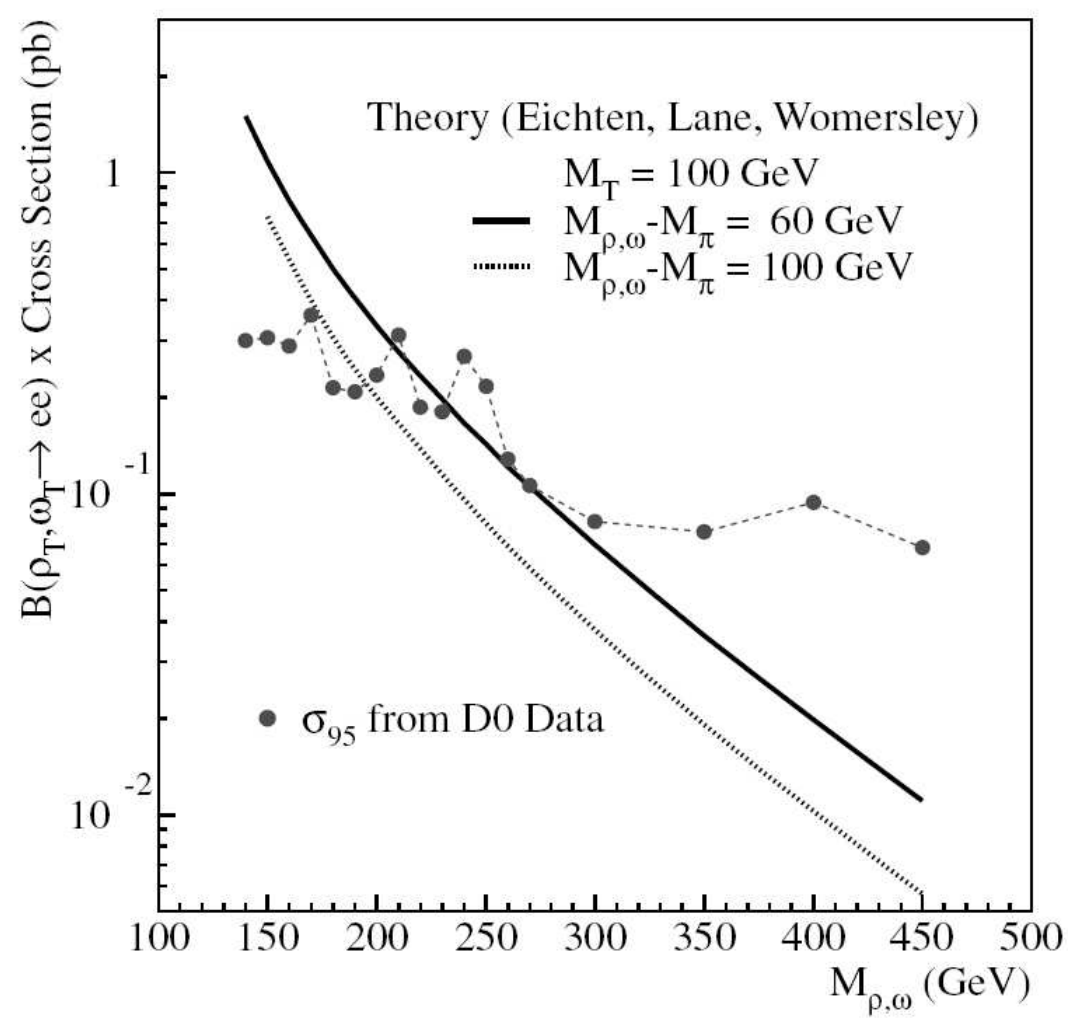

Figure 1.5: Cross section and 95\% confidence level upper limit for the production of $\rho_{T} / \omega_{T} \rightarrow e e$, as found by the D $\varnothing$ collaboration [16].

by the TCSM authors:

- $N_{T C}=4$ : the number of technicolors.

- $F_{T}=82 \mathrm{GeV}$ : the technipion decay constant. With $F_{T} \sqrt{N_{D}}=246 \mathrm{GeV}$, this implies the number of techniquark doublets $N_{D}=9$.

- $\sin \chi=\frac{1}{3}$ : the gauge boson-technipion mixing parameter.

- $Q_{U}=\frac{4}{3}$ : the charge of the up type techniquark.

- $Q_{D}=\mathrm{Q}_{D}=Q_{U}-1=\frac{1}{3}$ : the charge of the down type techniquark.

- $M_{V}=M_{A}$ : the mass parameters were varied from $100 \mathrm{GeV}$ to $500 \mathrm{GeV}$. This does not change any kinematic distributions, but does alter the production cross-sections. 

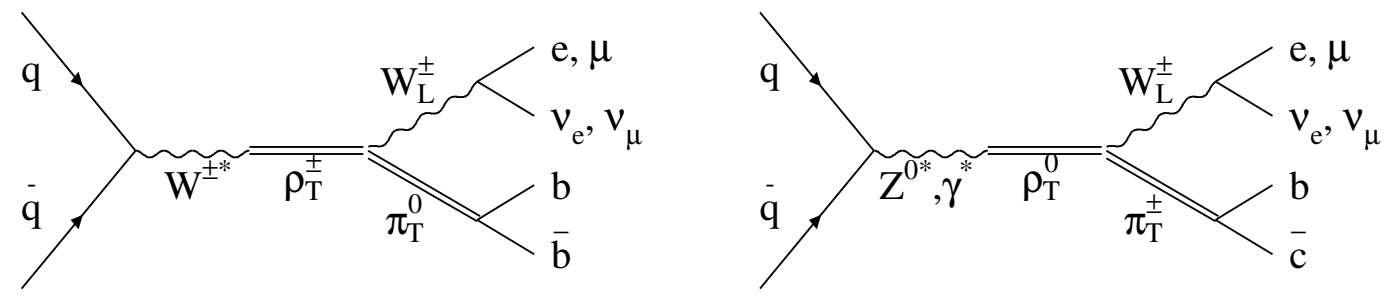

Figure 1.6: Feynman diagrams for the production of Technicolor particles at the Tevatron.

No direct assumption is made about the Technicolor interaction strength. Any information about its value is encoded in parameters such as $F_{T}$. 


\section{Chapter 2}

\section{Experimental Apparatus}

The Tevatron accelerator complex, shown schematically in Figure 2.1, collides protons and antiprotons at a center of mass energy of $1.96 \mathrm{TeV}$. The $\mathrm{D} \varnothing$ detector is one of two general purpose detectors that monitor the results of these collisions. In this chapter, an overview of the Tevatron is provided, followed by discussion of the $\mathrm{D} \varnothing$ detector.

\subsection{The Tevatron Accelerator Complex}

The acceleration process begins with the production of negatively charged hydrogen ions which are injected into the Cockroft-Walton accelerator. Here the ions are passed through an electrostatic field and are injected, with an energy of $750 \mathrm{KeV}$, into a linear accelerator (LINAC). In the LINAC, they are accelerated by means of RF fields to an energy of $400 \mathrm{MeV}$. At this stage, a beam of protons is produced from the hydrogen ions by passing them through a carbon foil, which strips off their electrons. The protons are subsequently transferred into the Booster synchrotron ring, and accelerated to $8 \mathrm{GeV}$. The protons are transferred again to the Main Injector which in turn accelerates them to $150 \mathrm{GeV}$. The Main Injector, feeds the Tevatron accelerator; additionally protons are extracted for the production of anti-protons.

One bunch (each consisting of $5-6 \times 10^{10}$ protons) is extracted from the Main Injector every 1.47 seconds to impact on a nickel-copper target, producing anti-protons as well as other secondary particles. The anti-protons are collected, bunched and accelerated to $8 \mathrm{GeV}$ in the Accumulator. They are then transferred to the Main Injector and accelerated to $150 \mathrm{GeV}$, travelling

in the opposite direction to the proton beam. Both protons and anti-protons are extracted to the Tevatron, which accelerates them to $980 \mathrm{GeV}$. In the Tevatron, the beams are brought together for collisions at the $\mathrm{D} \varnothing$ and $\mathrm{B} \varnothing$ 


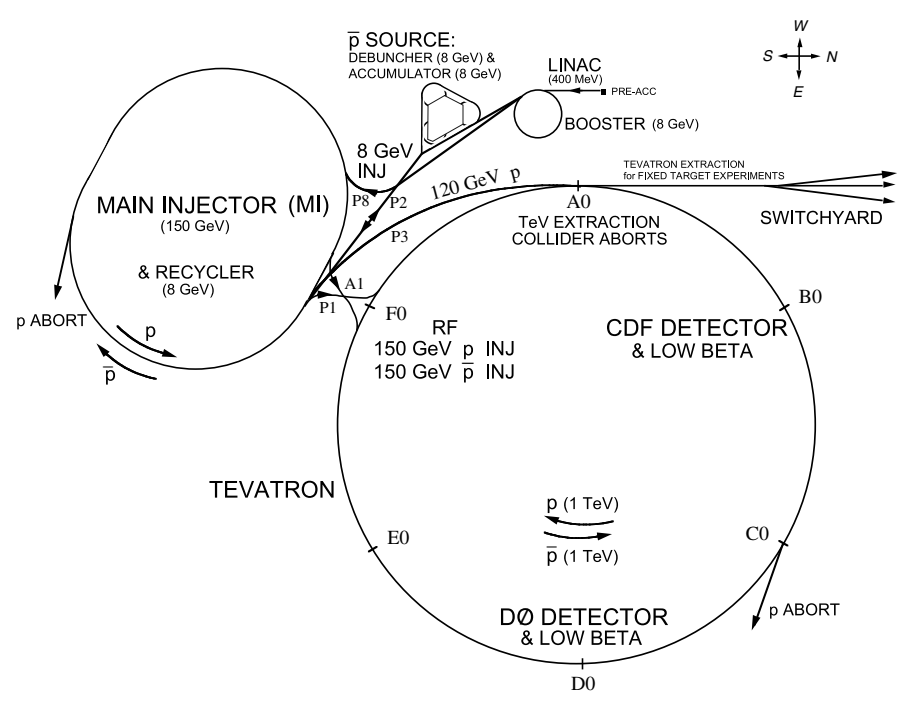

Figure 2.1: The Tevatron Complex at the Fermi National Accelerator Laboratory $[20]$.

interaction regions, which are the locations of the D $\varnothing$ and CDF detectors, respectively. The Tevatron complex is described in more detail in reference $[20]$.

\subsection{The DØ Coordinate System}

A right-handed coordinate system is used at $\mathrm{D} \varnothing$, with the $z$-axis aligned along the beam-pipe. At this point along the ring, the protons travel south, which defines positive $z$. Positive $x$ points east, implying that positive $y$ is up. In addition to the common rectilinear and spherical coordinates, $\mathrm{D} \varnothing$ frequently uses a modified spherical system system in which the polar angle $\theta$ is replaced with the pseudorapidity $\eta$, defined as:

$$
\eta \equiv-\ln \left(\tan \left(\frac{\theta}{2}\right)\right)
$$

It is useful to note that $\eta$ and $z$ share the same sign, and that the $\eta=0$ and $z=0$ planes are the same. In general the term central refers to regions with low $|\eta|(\lesssim 1.2)$, while forward (or end) means regions with high $|\eta|$. This coordinate, illustrated in the cross-section of the $\mathrm{D} \varnothing$ tracking system shown in Figure 2.2, is useful because the $\eta$ distribution of particles produced by purely QCD interactions is flat. The true rapidity: 


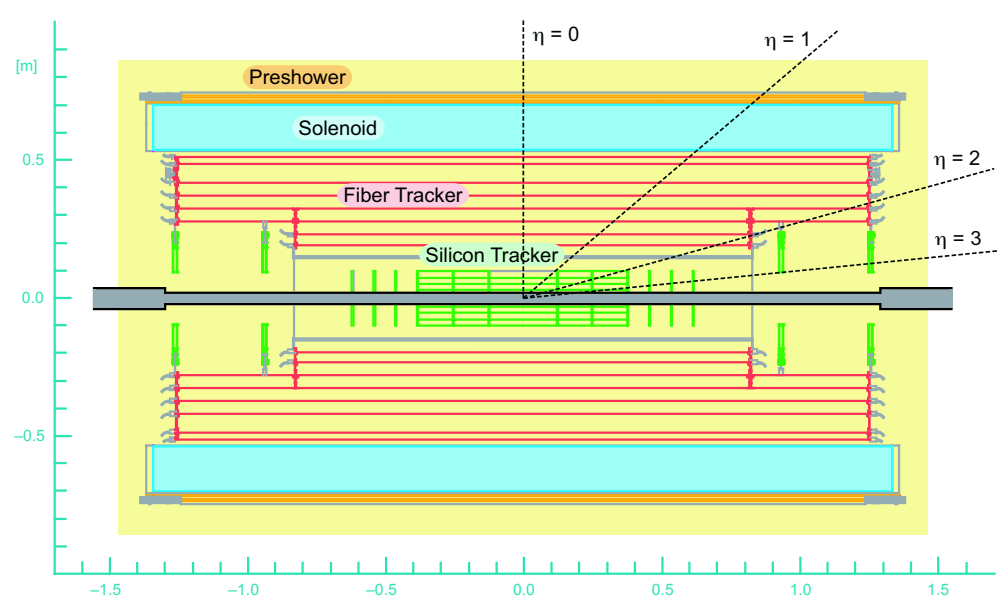

Figure 2.2: Cross section of the DØ inner tracking system [21].

$$
Y \equiv \frac{1}{2} \ln \left(\frac{E+p_{z}}{E-p_{z}}\right)
$$

is also used in the reconstruction of jets. In the massless limit typical for the final state particles at Tevatron energies, $Y \approx \eta$.

It is normally convenient to define the coordinate system with respect to the location of the actual interaction point, which varies from event to event. However, it is also useful to define an absolute coordinate system, with the detector center as its origin. These are referred to as the physics and detector coordinates respectively, and appropriate subscripts are used as neccessary. With the exception of this chapter, physics coordinates are assumed in the absence of any subscripts. Here the situation is reversed.

\subsection{The DØ Detector}

The high energies and luminosities at the Tevatron make possible the study many rare processes at the smallest distance scales currently accessible. The $\mathrm{D} \varnothing$ detector is designed to identify the high energy particles produced by these processes. A cross-section of the detector is shown in Figure 2.3. Individual charged particles deposit energy in a tracking system that is enclosed in a solenoidal magnet with a field strength of 2 Tesla. Togther, they enable the measurement of particle momenta and aid in particle identification. Preshower detectors and a liquid-argon/uranium calorimeter provide measurements of 


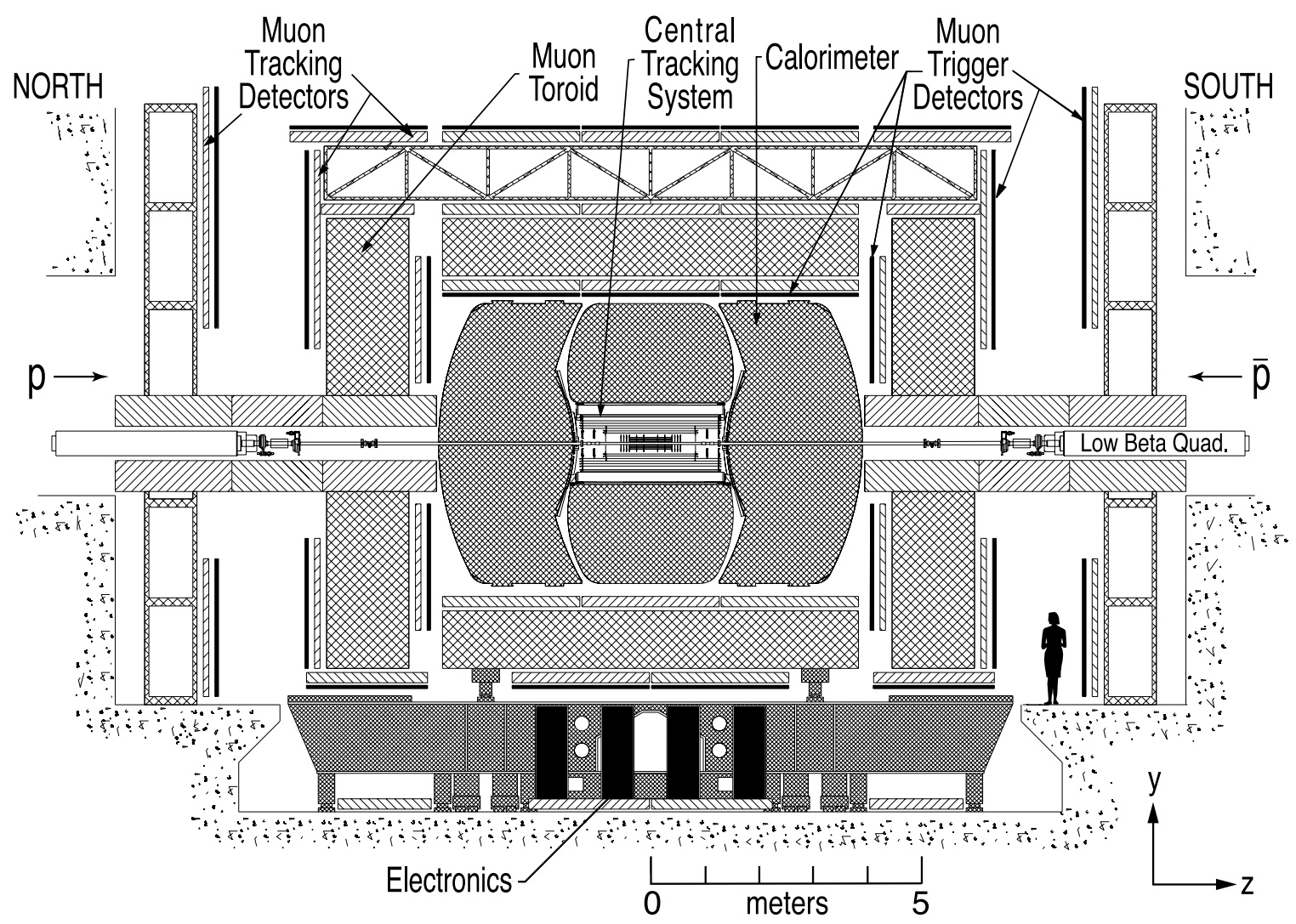

Figure 2.3: Overview of the $\mathrm{D} \varnothing$ detector.

the energies of most particles. Finally, a system composed of drift tubes and scintillators, as well as a 1.8 Tesla toroidal magnet, is used to identify muons and provide an additional, lower resolution, measurement of muon momenta. The rate of collisions at the Tevatron is many orders of magnitude too large for all of them to be recorded. A three-tiered trigger and data acquisition system selects which collisions to save.

The detector, along with the low-level trigger and readout electronics, is enclosed in an underground collision hall. Access is normally restricted because of the radiation produced when the Tevatron is active. The remainder of the electronics, trigger and data-acquisition systems are located in movable and fixed counting houses that are adjacent to the collision hall.

For further information on the $\mathrm{D} \varnothing$ detector, the reader is directed to consult References [22] and [23]. Except where noted, all information and figures in this chapter are taken from these references. 


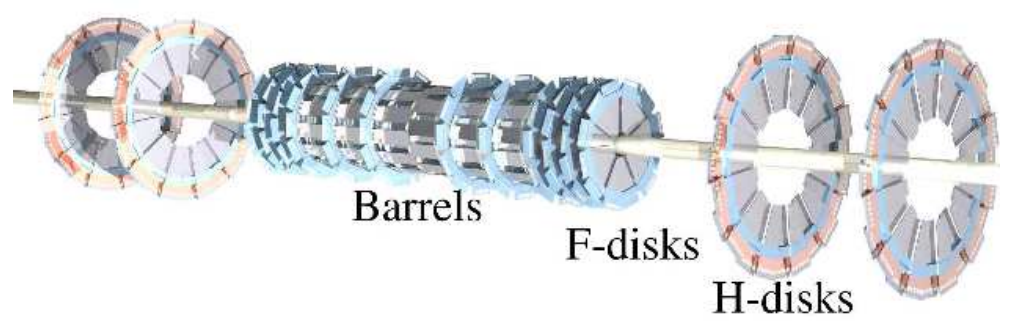

Figure 2.4: The Silicon Microstrip Tracker.

\subsubsection{The Silicon Microstrip Tracker}

Mounted directly on the Tevatron's beryllium beampipe is the Silicon Microstrip Tracker (SMT), which is used for high precision track and vertex reconstruction. The SMT is composed of wafers of silicon containing strips typically 50 microns in pitch doped to form semiconducting traces. A charged particle traversing such a sensor generates electron-hole pairs that are collected by cathode strips deposited onto the wafer surface. The resulting electrical pulses are digitized by by SVXII-e chips. It is possible to create double-sided sensors by doping strips on both sides of the wafer, producing p-type strips on one side and n-type strips on the other, usually at some angle to the the first side. Tracks may be reconstructed in three dimensions through the use of this stereo information.

The SMT, shown in Figure 2.4, is composed of six barrels, twelve F-disks and four H-disks. As shown in Figure 2.2, the SMT provides tracking coverage for $|\eta|<3$. The $\mathrm{F}$ disks are composed of double sided sensors with strips of one layer at a $30^{\circ}$ stereo angle to the strips of the other. The $\mathrm{H}$ disks consist of a pair of single sided devices mounted back to back at a $15^{\circ}$ stereo angle.

The barrels have four layers of sensors in three configurations. The second and fourth layers of all barrels consist of double sided sensors with a $2^{\circ}$ stereo angle. Layers one and three of the inner four barrels are composed of double sided sensors with a $90^{\circ}$ stereo angle. In the outer two barrels, layers three and four are composed of single-sided devices with the strips oriented axially (i.e., along the $z$-axis). One side of the stereo devices is always oriented axially.

The SVXII-e read-out chip is a radiation-hard 128 channel analog pipeline and 8-bit ADC that provides channel-specific thresholds to allow real-time zero suppression, thus minimizing readout times. These chips are daisy-chained in groups of up to nine on Kapton flex circuits. ${ }^{1}$ Individual channels are con-

\footnotetext{
${ }^{1}$ Kapton is a radiation-hard plastic frequently used in high energy physics exper-
} 


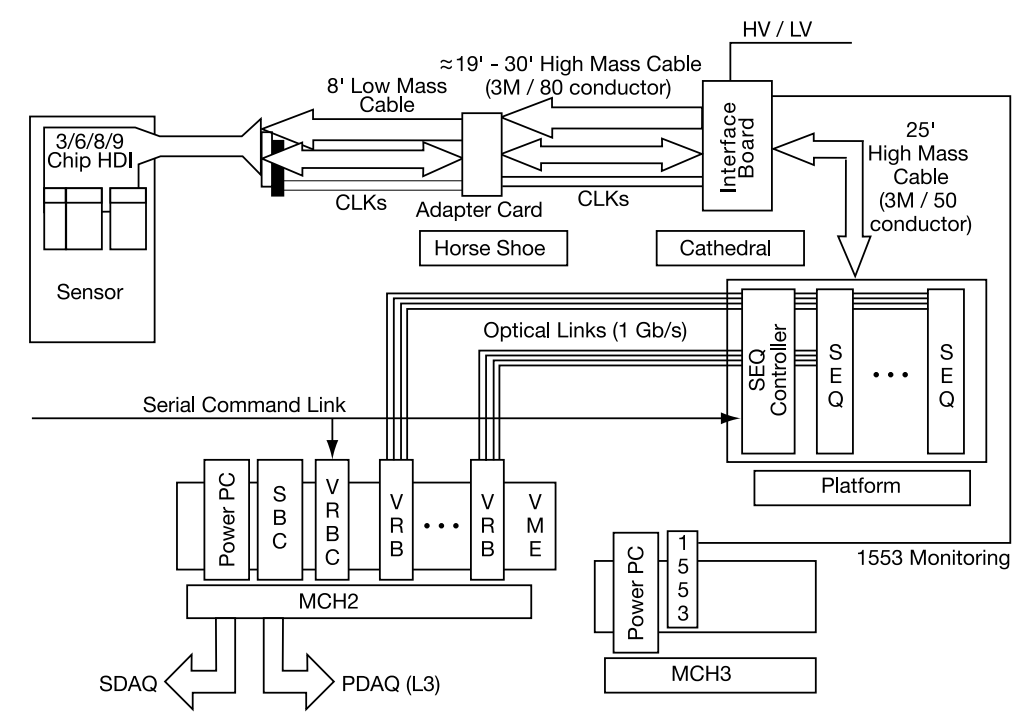

Figure 2.5: Schematic of the SMT readout.

nected to the SMT cathodes. The flex circuits are connected by low mass cables to adapter cards outside of the calorimeter. These are connected by high mass cables and additional interface boards to sequencer boards on the electronics platform below the detector. The sequencers provide timing and control signals to the SVXII-e chips, and are connected by optical fibers to VME Readout Buffers (VRBs) in VME (Versa Module Eurocard) crates outside of the collision hall. The adapter card interface board chain serves to collect data from and distribute control signals and power to the SMT sensor assemblies. This readout chain is shown in Figure 2.5.

An initial (Level 1) trigger accept is delivered by serial command link (SCL) from the trigger framework to the sequencers, which distributes it to the SVXII-e chips. On receiving a trigger, the chips extract the analog signals from the pipelines and, if above threshold, digitize them. The digital information is read out by the sequencers and transmitted to the VRBs, where it is buffered, awaiting a confirmation from the second tier of the trigger system. If and when this is received a single board computer (SBC), in each VME crate collects the data via the crate backplane and transmits it via ethernet to the trigger system.

iments. 


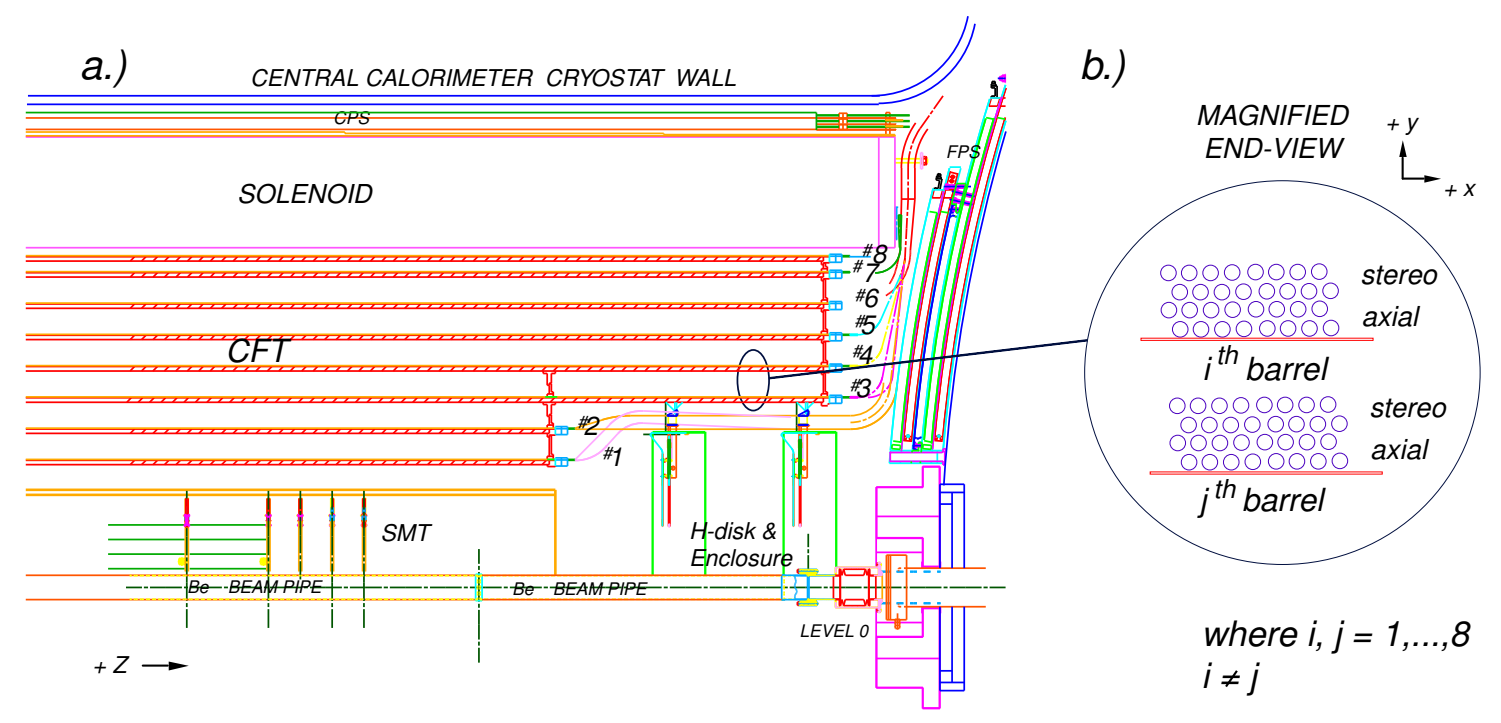

Figure 2.6: The central fiber tracker [24].

\subsubsection{The Central Fiber Tracker}

The Central Fiber Tracker (CFT), shown in Figure 2.6 consists of 76,800 scintillator-doped fibers mounted on eight concentric cylinders surrounding the SMT. A charged particle traversing these fibers ionizes their organic molecules. The excited molecules emit photons as they return to their ground states. This light is captured by clear optical fibers attached to one end of each scintillating fiber. The end not connected to the waveguide is mirrored to increase the total light collected. These optical signals are delivered to visible light photon counters (VLPCs) that convert them to electrical signals, which are then elivered to the trigger and data acquisition systems.

Each cylinder of the CFT consists of one layer of axial fibers immediately surrounded by another layer of stereo fibers which are at an angle of $3^{\circ}$ with respect to the beam axis. The sign of the angle alternates with each successive stereo layer. The innermost two layers provide coverage of up to $|\eta|<1.6$, while the remaining layers provide coverage up to $|\eta|<1.8$.

The VLPCs are avalanche photodiodes consisting of arsenic-doped silicon. These devices typically have gains of 22,000 - 65,000 and quantum efficiencies of in excess of $75 \%$. The optimal operating temperature for a VLPC is approximately $9 \mathrm{~K}$ and varies from chip to chip. The temperature is precisely maintained by suspending the VLPCs over a liquid helium bath in conjuction with the use of resistive heaters. The electrical pulses are delivered to the 
readout electronics on flexible cables consisting of gold traces deposited on Kapton.

The signals from the VLPCs are processed by analog front end (AFE) boards which provide a list of hits to the trigger system (for the axial layers only) and an ADC readout to the DAQ. A pulse is delivered to a SIFT discriminator chip which, produces the trigger information, and subsequently to an SVXII-e chip for zero suppression and digitization. Since this is the same ADC chip used in the SMT, the remainder of the readout chain for the CFT also uses the same architecture.

\section{The Level 1 Central Track Trigger}

The list of discriminator hits from the axial fibers is transmitted to the data acquisition system formatted as the readout information from an SVXII-e chip. A bitmask identifying all the hits is also sent over Low Voltage Differntial Signal (LVDS) cables to the central track trigger (CTT). The CTT is composed of a series of of digital front end (DFE) boards connected by LVDS cables. It rapidly identifies tracks for use in the first two levels of the trigger system by comparing the list of hits to a set of $\sim 20,000$ pre-loaded track definitions.

The bitmasks are first transmitted to mixer boards that map the hits in 80 trigger sectors, each $4.5^{\circ}$ wide in $\phi$. The remapped hits are transmiitted to the first stage (DFEA) boards for track finding. The tracks are grouped in four $p_{T}$ bins: $1.5-3 \mathrm{GeV} / c, 3-5 \mathrm{GeV} / c, 5-10 \mathrm{GeV} / c$, and $>10 \mathrm{GeV} / c$. The axial layer of the central preshower (see section 2.3.3) is searched for clusters produced by electrons showering in the solenoid and the lead mounted around it. The list of tracks is transmitted to the Level 1 muon trigger to form muon candidates. The track counts, categorized by $p_{T}$ bin, track isolation, and the presence of a preshower match are sent to the next stage (CTOC) boards which each collect and sum the track counts from ten trigger sectors. Finally, a single third stage (CTTT) board calculates elementary trigger conditions (trigger terms) that are transmitted to the Level 1 framework. The input data from the second and third stage DFEs are buffered for transmission for read-out on a Level 1 accept.

\subsubsection{The Central and Forward Preshowers}

The central and forward preshowers each consist of nested triangular strips of scintillating plastic embedded with wavelength shifting (WLS) fibers, as shown in Figure 2.7. A charged particle traversing such a layer induces blue light in the scintillator. This light excites a transition in the WLS fibers that 


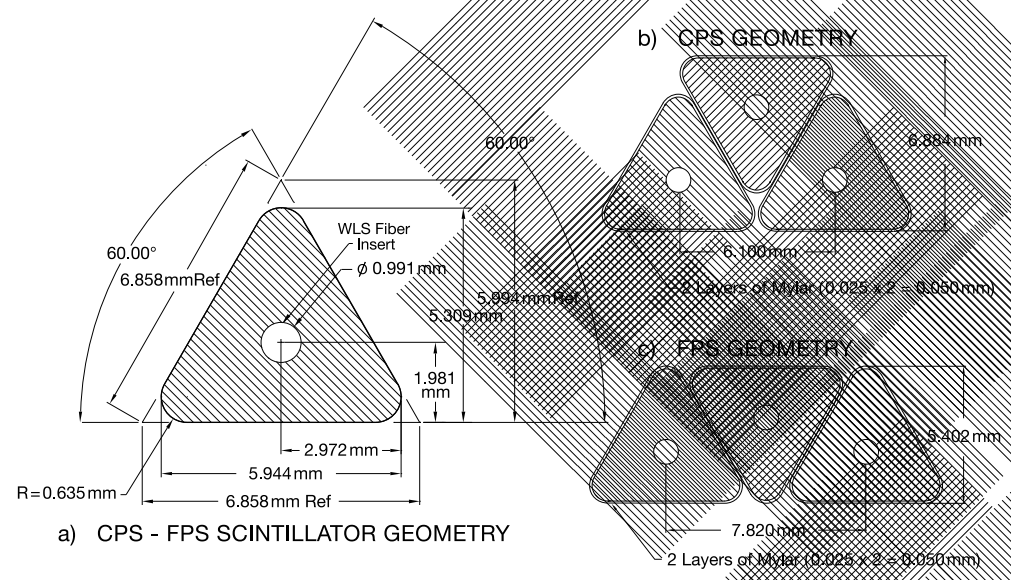

Figure 2.7: Cross section of the scintillating strips used in the CPS and FPS. The circles indicate the location of the embedded wavelength shifting fibers.

produces green light, which is read out by the same VLPC system used by the CFT. The nested triangular geometry of the preshower detectors is such that a particle will typically pass through multiple strips, and the relative energy deposition in each will give a precision measurement of the particle's position that is much finer than the granularity of the detector.

The central preshower detector (CPS) consists of three layers of scintillating strips mounted in concentric cylinders around a lead cylinder, itself mounted on the $\mathrm{D} \varnothing$ solenoid. The strips in the innermost (axial) layer of the CPS are parallel to the beampipe while the outer two ( $u$ and $v$ ) stereo layers are at angles of $\pm 22.5^{\circ}$ with respect to the innermost layer. This stereo geometry allows the reconstruction of three-dimensional clusters. To simplify the combinatorics of any clustering algorithm, the CPS is segmented at $z=0$ into two sections.

A forward preshower (FPS) detector is mounted on each of the calorimeter endcaps. As shown in Figure 2.8, the FPS consists of two layers of trapezoidal modules separated by two radiation lengths of lead. Each module is composed of two sublayers ( $u$ and $v$ ) of scintilliating strips at a stereo angle of $22.5^{\circ}$ and covering $22.5^{\circ}$ in azimuth.

A particle leaving the interaction region first encounters the MIP layer, so called because charged particles traversing it behave as minimally ionizing particles (MIPs) and deposit only a small amount of energy. The shower layer is mounted directly on the calorimeter; it is encountered only after a particle passes through the electromagnetic shower inducing lead. The MIP 


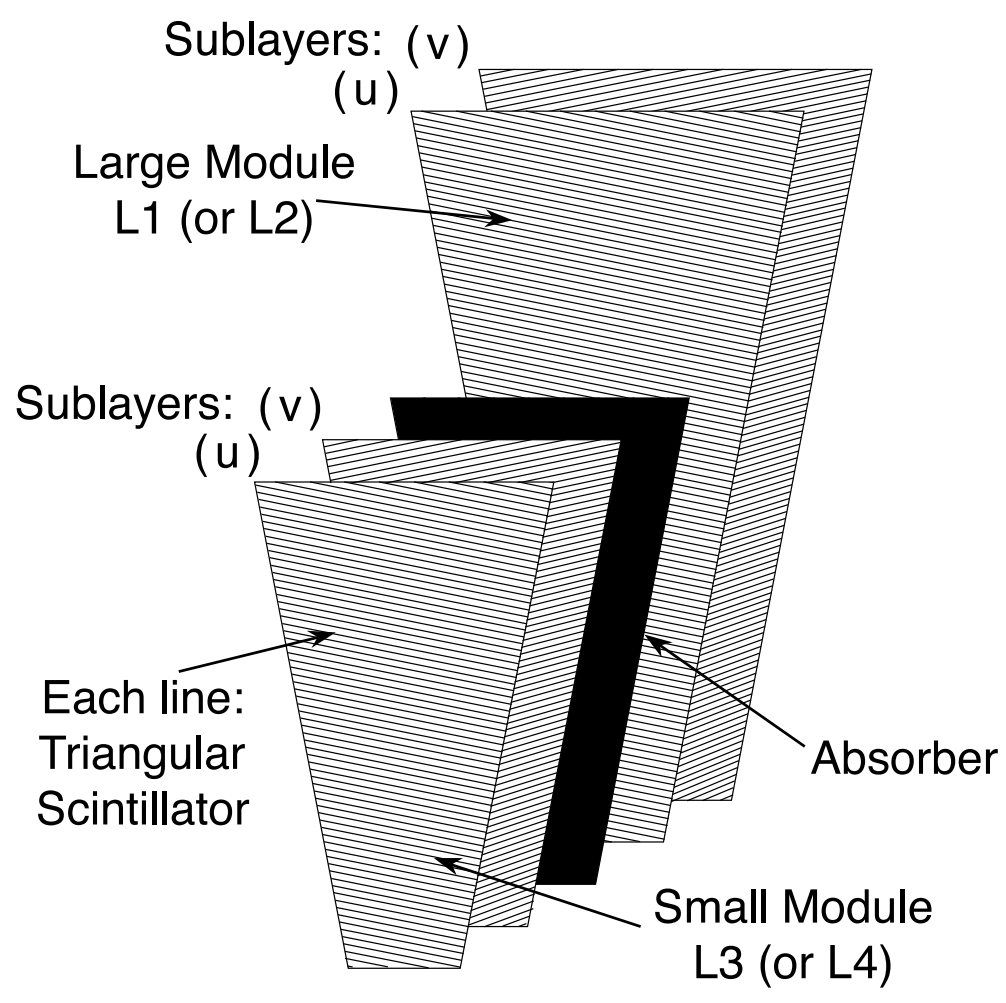

Figure 2.8: Exploded view of MIP and shower layer modules, separated by two radiation lengths of lead.

layer covers $1.65<|\eta|<2.5$, while the shower layer covers $1.6<|\eta|<2.5$.

Discriminator hits from both the preshowers are used in the trigger. The stereo hits from the CPS are used only at Level 2, while the axial CPS hits and all of the forward preshower hits are also used at Level 1.

\subsubsection{The Solenoid}

To enable the measurement of charged particle momenta, a $2 \mathrm{~T}$ solenoid is installed between the CFT and the CPS. The magnetic field is held constant to about $5 \%$ within the inner tracking volume. To keep the field uniform at the ends of this volume, the current density is increased by use of a thinner conductor. The solenoid is constructed with superconducting material that is cooled by liquid helium. The magnet is $2.73 \mathrm{~m}$ long and has an exterior diameter of $1.42 \mathrm{~m}$. The interior diameter is $1.067 \mathrm{~m}$. The full solenoid assembly is approximately 0.87 radiation lengths thick. When active, the 


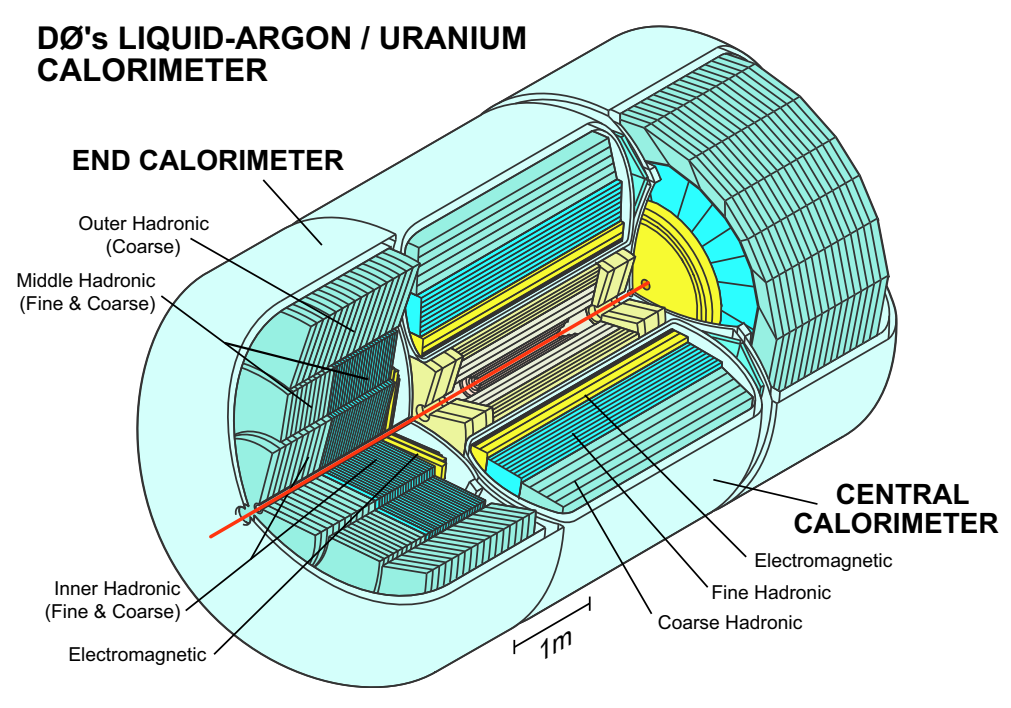

Figure 2.9: The D $\varnothing$ calorimeter.

energy stored in the magnetic field is 5.3 MJ.

\subsubsection{The Calorimeter}

The D $\varnothing$ calorimeter, shown in Figure 2.9, provides energy measurement of most long-lived particles, and enables for the efficient identification of jets, electrons, and photons. It consists of a central cryostat (CC), covering $|\eta|<1.1$, and two endcap cryostats (EC), covering $1.3<|\eta|<4$. Two intercryostat detectors, consisting of scintillating tiles that are read out by photomultiplier tubes, give additional coverage in the gaps between the cryostats. The cryogenic and electrical services for the solenoid as well as the cabling for the inner tracking system are routed between the $\mathrm{CC}$ and the ECs.

The calorimeter is composed of cells containing liquid argon, grounded absorber plates (see Table 2.1) and high voltage pads. A typical cell is shown in Figure 2.10. A particle traversing the calorimeter will interact with absorber, producing a shower of secondary particles. These secondary particles ionize the liquid argon and the resulting charge is collected at the high voltage pads, giving a measurement of the energy deposited in that cell. The low energy neutrons liberated by nuclear breakup (caused by hadronic showers) induces fission in the depeleted uranium, which compensates for the lower visible energy in such showers. The electromagnetic and hadronic responses of the calorimter are further equalized by the choices of the absorber composition 


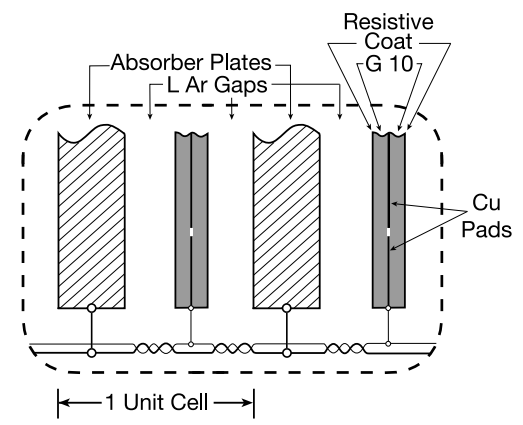

Figure 2.10: Schematic view of a typical calorimter unit cell. The signal boards are the copper pads with resistive coats.

\begin{tabular}{|c|c|c|c|}
\hline Layer type & EM & Fine Had. & Coarse Had. \\
\hline \multicolumn{4}{|c|}{ Central } \\
\hline Absorber & Uranium & $\mathrm{Ur}(2 \% \mathrm{Nb})$ & Copper \\
\hline Layers & 4 & 3 & 1 \\
\hline EM radiation lengths & $2.0,2.0,6.8,9.8$ & & \\
\hline Nucl. interaction lengths & 0.76 total & $1.3,1.0,0.9$ & 3.2 \\
\hline \multicolumn{4}{|c|}{ EndCaps } \\
\hline Absorber & Uranium & $\mathrm{Ur}(2 \% \mathrm{Nb})$ & Stainless Steel \\
\hline Layers & 4 & 4 & 1 \\
\hline EM radiation lengths & $2.0,2.6,7.9,9.3$ & & \\
\hline Nucl. interaction lengths & 0.95 total & $1.1(0.9)$ each & $4.1(4.4)$ \\
\hline
\end{tabular}

Table 2.1: Details of the calorimter cell composition. The radiation and interaction lengths are listed by layer. In the case of the EC the nuclear interaction lengths are quoted separately for the Inner Hadronic and Middle Hadronic sections (the latter being the parenthetical figures).

and thickness.

The calorimeter cells are arranged in four electromagnetic layers, three fine hadronic layers (four in the ECs) and one coarse hadronic layer. The absorbers in the electromagnetic layers are made of depleted uranium. The fine hadronic absorbers are composed of a uranium-niobium alloy and the coarse hadronic absorbers consist of copper (in the CC) and stainless steel (in the ECs). The EC hadronic layers are further divided into the inner, middle and hadronic sections, shown in Figure 2.9.

The calorimeter cells all subtend an area of $0.1 \times 0.1$ in $\eta-\phi$ space, except 


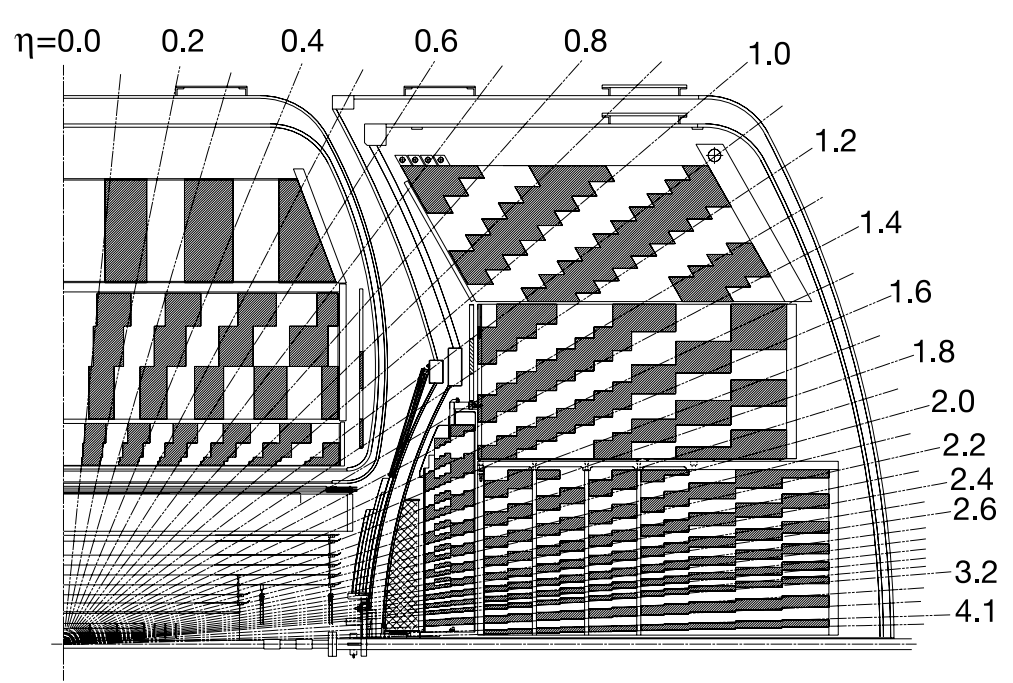

Figure 2.11: Cross-section D calorimeter.

for the third electromagnetic layer, which has twice the segmentation in both dimensions. Details of the cell composition are shown in Table 2.1. The showers from electrons and photons stop in the fourth electromagnetic layer with a maximum in the third layer. The finer segmentation of this layer allows a more precise measurement of the shower position. The cells are aligned in projective towers (visible in Figure 2.11) with $\eta-\phi$ dimensions of $0.2 \times 0.2$. The total energy in these towers is read out for use in the Level 1 and 2 trigger systems.

The drift time of ions in the liquid argon is $\sim 450 \mathrm{~ns}$, while the time between collisions is $396 \mathrm{~ns}$. Therefore the charge collected from previous events piles up in the readout of the current event. This problem is solved by a method called baseline subtraction, in which the charge from the preceding collision is sampled and subtracted from the signal in the event being read out.

\subsubsection{The Muon System}

The D $\varnothing$ muon system is a toroidal spectrometer consisting of drift tubes and scintillating tiles. Exploded views of both are shown in Figures 2.12 and 2.13. The system contains three layers (called A, B and C) and is segmented into one central $(|\eta|<1.1)$ and two forward $(1.1<|\eta|<2.0)$ regions. 


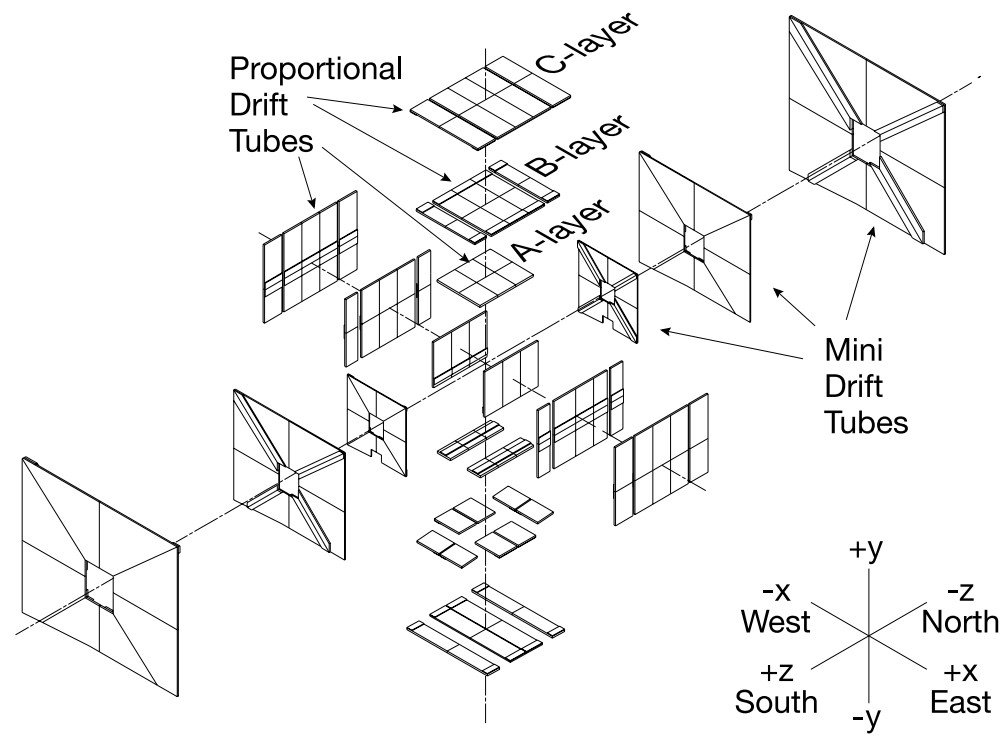

Figure 2.12: Exploded view of the D $\varnothing$ muon drift tubes.

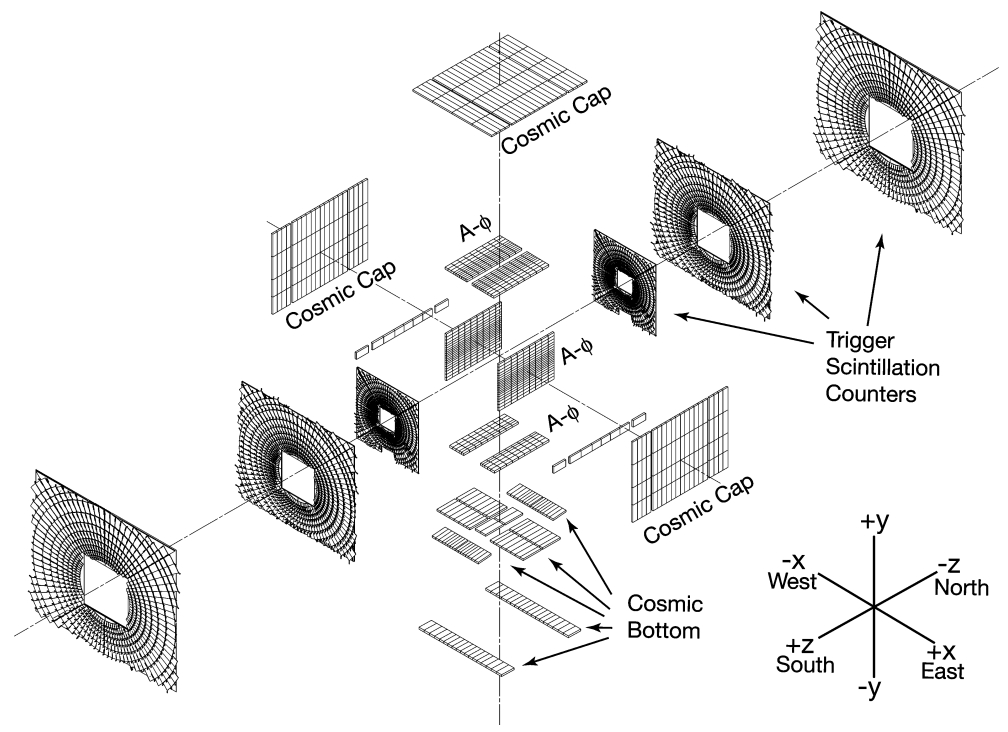

Figure 2.13: Exploded view of the D $\varnothing$ muon scintillators. 
The A layer is directly outside of the calorimeter, and it is surrounded by a toroidal magnet with an iron yoke. The iron yoke suppresses hadronic backgrounds which punch through the calorimeter and stops muons with $p_{T} \lesssim$ $3 \mathrm{GeV} / c$. Additional shielding in the very forward regions, shown in Figure 2.3, reduces backgrounds in the forward muon system arising from stray particles associated with the colliding beams. Outside of the toroid are the B and $\mathrm{C}$ layers. Muons passing through the toroid are deflected by the 1.8 Tesla magnetic field providing an measurement of their momentum independent of the central tracking system. A hole in the bottom A layer is neccessary for the structual support of the calorimeter. This prevents triggers for downward going muons (specifically, those with $|\eta|<1.0$ and $4.25<\phi<5.15$ ). It is possible to reconstruct muons in this region, but only with substantially reduced efficiency.

The proportional drift tubes (PDTs) used in the central muon system are $254 \times 559 \mathrm{~cm}^{2}$ chambers constructed of extruded aluminum tubes. Each PDT is composed of $10.1 \mathrm{~cm}$ cells and is 24 cells wide and are either three or four cells long. The A layer contain four decks (i.e., sublayers) of PDTs, except at the bottom of the detector where it consists of three decks. The $\mathrm{B}$ and $\mathrm{C}$ layers each consist of three decks of PDTs. Approximately $55 \%$ of the central region is covered by all three layers of PDTs, while the coverage for at least two layers is $90 \%$.

The forward muon system uses three layers of mini drift tubes (MDTs) and scintillators. Each layer of MDTs is divided into octants, visible in Figure 2.12. An octant assembly consists of three (for the B and $\mathrm{C}$ layers) or four (for the A layer) planes of tubes oriented along the toroid field lines. Each tube consists of eight cells, $9.4 \times 9.4 \mathrm{~mm}^{2}$ in cross-section, running the length of the tube.

Light from the scintillation tiles is collected by photomultiplier tubes that provide a fast readout for triggering. The time of the scintillator hit relative to the beam crossing time is also recorded, which is used to reject cosmic rays and backgrounds arising from the decay radioactive materials in the collision hall (e.g., uranium in the calorimeter). The central A layer tiles ( $A \phi$ counters) are 33.25 " long and have widths of 14.46 ", 10.84" or 9.09". The B and C layer tiles (the cosmic cap and bottom counters) are 25" wide and 81.5" - 113" long. All tiles subtend an angle of $4.5^{\circ}$ in $\phi$ and match the trigger sectors of the central track trigger. The forward scintillion tiles also match the CTT $\phi$ segmentation and have an $\eta$ coverage of 0.12 in the innermost nine rows of counters, and 0.7 for the last three rows. 


\subsubsection{The Trigger and Data Acquisition Systems}

Collisions occur at the $\mathrm{D} \varnothing$ detector once every $396 \mathrm{~ns}$, a rate of $2.5 \mathrm{MHz}$. Considerations such as the rate at which data can be recorded on data storage tapes, the cost of such tapes and the amount of time required to reconstruct recorded events dictate that events be saved at a rate of only $50 \mathrm{~Hz}$. To accomplish this goal while retaining with high probability collisions deserving of further study (e.g., events containing W or Z bosons), a three-tiered trigger system is used. The first tier, or Level 1 (L1), is based on custom-made hardware and firmware. It reduces the rate to $\sim 2 \mathrm{kHz}$. The Level 2 (L2) trigger runs simple software algorithms on a series of custom-made single board computers and further reduces the rate to $\sim 1 \mathrm{kHz}$. The Level 3 (L3) trigger system runs more sophisticated algorithms on a dedicated farm of rack-mounted computers. It is tightly integrated with the $\mathrm{D} \varnothing$ data acquisition system (DAQ) and reduces the rate to the required $\sim 50 \mathrm{~Hz}$.

\section{The Level 1 Trigger}

While awaiting a Level 1 trigger decision, event data is buffered in the front end electronics (e.g., in the SVXII-e chips for the tracking and preshower detectors). The data are read out from all subsystems except the SMT with reduced precision, and transmitted to specialized hardware. Algorithms implemented in firmware process the data, calculate trigger terms and send the results to the trigger framework. The trigger framework forms more complictated triggers by using AND operations on the various trigger terms. An event satisfying any of these requirements is accepted and passed to the Level 2 trigger.

The Level 1 calorimeter trigger examines the energy deposited in the projective towers discussed in section 2.3.5 (also referred to as trigger towers). Trigger terms are formed by counting the number of towers with total energy exceeding one of a set of preprogrammed thresholds. For example, one could require for three towers with total energy above $5 \mathrm{GeV}$ or one tower with a total energy of $10 \mathrm{GeV}$. The energy in the electromagnetic layers are compared to a separate set of thresholds.

The CTT, to repeat in brief the discussion in section 2.3.2, searches the CFT for tracks exceeding predefined $p_{T}$ thresholds. Isolated tracks are identified as well as tracks with matching CPS hits. The shower layer of the forward preshower is also searched for clusters with and without matching MIP layer hits. Trigger terms are formed by requiring $u$ and $v$ layer clusters in the same quadrant, but no attempts are made to match them with finer resolution. 
Conceptually, the Level 1 muon trigger functions much like the CTT, comparing hits in the detector to preloaded roads (i.e., possible trajectories for the muons produced in collisions). Backgrounds from cosmic rays and spurious hits due to the radioactive environment are rejected by requiring that the timing of the hits be consistent with muons originating from a beam crossing. It is also possible to match muon hits to tracks from the CTT, providing the only muon $p_{T}$ measurement available at Level 1 .

\section{The Level 2 Trigger}

The reduced precision data available at Level 1 are supplemented with additional data on specialized hardware such as the DFE boards used in the CTT. These results are transmitted to preprocessors installed on single board computers (referred to as L2 $\beta$ boards) where fast reconstruction algorithms generate crude physics objects that are sent on to a global processor, also running on a $L 2 \beta$ board. The global processor correlates the results from the various sub-detectors and applies different predefined trigger requirements, depending on which Level 1 triggers the event satisfied.

The Level 2 calorimeter preprocessors search the calorimeter for seed towers that are used as starting points to build $n \times n$ tower clusters for triggering. These seeds are trigger towers with a transverse energy above some threshold. Jets are reconstructed as $5 \times 5$ clusters with a seed of $E_{T}>2 \mathrm{GeV}$. Electromagnetic objects, such as electrons and photons, are built from seeds with $E_{T}>1 \mathrm{GeV}$ in the EM layers of the calorimeter and consist of $3 \times 3$ clusters. An event $\not_{T}$ is also calculated.

On receiving a L1 accept, the DFE boards used in the L1CTT transmit a $p_{T}$ sorted list of tracks to the Level 2 CTT preprocessors and the silicon track trigger (STT). The sorting permits the use of faster algorithms, and minimizes the impact of any truncation of the list that may be necessary. This track list has a much finer $p_{T}$ resolution than that used in the Level 1 decision. The SMT data coming out of the sequencers are duplicated and sent to the STT as well. The STT uses the tracks from the CTT as seeds to find tracks in the $\mathrm{SMT}$. These tracks have impact parameter and refined $p_{T}$ measurements. The impact parameter measurements may be used for triggering on the displaced vertices found in $b$-jets. The resulting track lists are transmitted to the, L2CTT which refines the track $\phi$ and projects the track location into the calorimeter. An isolation is also calculated, based on the occupancy of the CFT near the track in question.

The stereo layer discriminator hits from the central preshower are supplemented with the axial hits used in the Level 1 trigger to form three-dimensional 
clusters. Likewise, the stereo hits from the FPS are also combined. The results from both preshowers are transmitted to the global L2 processor for correlation with the information from other detector components.

The muon candidates from Level 1 are refined with a calibrated readout and additional precision in the timing information. Track segments from the A layer are combined with those from the $\mathrm{B}$ and $\mathrm{C}$ layers to provide momentum measurements. Muon $\eta$ and $\phi$ coordinates are provided, and a quality value based on the number of hits is assigned.

\section{The Level 3 Trigger and DAQ}

Prior to a L2 accept, the detector data are buffered in VME readout crates outside of the collision hall. When a positive trigger decision is receieved, a single board computer (SBC) in each crate collects the data from the buffers. The trigger framework assigns the event to a node from the Level 3 farm and directs each SBC to transmit its data to that node using gigabit ethernet cables. After the node has collected all of the event fragments from the readout crates, it runs a software reconstruction program optimized for speed on the full event. Events are selectively reconstructed based on which L1 and L2 trigger requirements have been satisfied; a final trigger decision is made based on the results. Accepted events are transmitted to an online host computer, which buffers the event on disk where it can be examined for the purposes of data quality monitoring. Finally, the event is saved on data storage tapes for later reconstruction. 


\section{Chapter 3}

\section{Event Reconstruction}

The raw data recorded by the $\mathrm{D} \emptyset$ data acquisition system consist primarily of digitized pulse-height results from the integration of collected charge, the light yields in scintillators, and the time differences from drift chambers. The DØReco program converts this basic information into useful physics quantities. The program applies calibration and alignment corrections to the data, runs cluster finding algorithms, converts positions from detector-specific to global coordinates and converts the pulse-heights to energy deposition measurements. Afterward. the final state particles and groups of particles (called jets) are reconstructed. Stricter algorithms are subsequently applied to reduce any remaining backgrounds and to apply final calibrations to energy and momentum measurements. Both sets of algorithms are described in this chapter.

During the first stage of the reconstruction process, charged particle tracks are identified by using data from the SMT and CFT. Vertices are then reconstructed from the intersections of these tracks, and the location of the primary collision is measured. This position is used to calculate the coordinates of all identified particles and to determine the missing transverse energy (missing $E_{T}$, or $E_{T}$ ). Electromagnetic objects (i.e., electrons and photons) and jets are found by searching for cones of energy deposited within the calorimeter, while muons are identified by combining tracks with hits in the muon system.

\subsection{Track Reconstruction}

The first step in the track reconstruction procedure is to cluster activated channels in the SMT and the CFT. The energy weighted centroids of the clusters are used as inputs for each of two tracking algorithms that run in parallel and form a pool of candidate tracks. Quality requirements, listed in Section 3.1.3, are applied to this pool to select the final list of tracks. 
The first track finding scheme is the Alternative Algorithm (AA), described in Section 3.1.1 [25]. The AA builds track candidates in the SMT and extrapolates them to the rest of the tracking system. After the initial pool of candidates has been filtered down (using the criteria from Section 3.1.3), the AA scheme is executed a second time to find tracks that originate in the CFT. During this stage, the preliminary vertices found in the track selection are used to reduce combinatoric backgrounds.

The Histogramming Track Finder (HTF) [26], detailed in Section 3.1.2, is the second tracking algorithm. The HTF finds tracks by filling a histogram of track parameter space (i.e., the track curvature and azimuthal angle) with values that are consistent with each hit in the CFT and the SMT. Physical tracks are then identified as local maxima in the histogram.

\subsubsection{The Alternative Algorithm}

The AA track reconstruction technique [25] builds track candidates from segments that have registered least three hits in the silicon microstrip tracker. The first hit on a track may occur in any SMT barrel or F-disk. Track segments are formed by searching for a second hit with $|\Delta \phi|<0.08$ in any silicon layer of greater radius. The third hit may be in any further SMT layer. The projection of these three points onto the $x-y$ plane is fit to a circle. The resulting track hypothesis is accepted if all of the following conditions are met:

- the fit $\chi^{2}$ is less than 16

- the radius of curvature is at least $30 \mathrm{~cm}\left(i . e ., p_{T}>180 \mathrm{MeV}\right)$

- the track circle passes within $2.5 \mathrm{~cm}$ of the beam spot.

The remaining silicon and fiber tracker layers are searched, in order of increasing radius, for hits to associate with the track candidate. The number of layers with missed hits is monitored, with adjustments made for dead or disabled channels. If multiple hits in the same layer match the candidate, then the hypothesis is split and each result considered separately. The process ceases for a given candidate once three contiguous missed hits occur.

Additional hits are associated with the track if the resulting $\chi^{2}$ increases by less than 16 for each new hit. The tracks are then subject to the quality requirements described in Section 3.1.3, as are the tracks that are found by the HTF algorithim. To locate tracks that have few or no hits in the SMT, the CFT is searched for seed hits. The combinatoric background is much worse than in the SMT because the CFT has no segmentation in the $z$-direction. 
This problem is controlled by considering only tracks that are consistent with a vertex that was identified in the track selection stage (see Section 3.1.3). Tracks are subjected to the same quality requirements, whether they were originated in the CFT or the SMT.

\subsubsection{The Histogramming Track Finder}

The Alternative Algorithm searches likely trajectories from a starting point defined by some initial hit. In contrast, the Histogramming Track Finder fills a histogram of the track parameters consistent with each hit [26]. The physical tracks in an event are thus associated with local maxima in the histogram, as shown in Figure 3.1.

The projection of a track onto the $x-y$ plane is charactarized by three parameters: the radius of curvature, $\rho=q B / p_{T}$ (where $q$ and $p_{T}$ are the charge and transverse momentum of the particle, and $B$ is the magnetic field); the impact parameter, $b$; and the azimuthal angle, $\phi_{0}$. For the tracks produced in the $p \bar{p}$ collisions under study, $b \approx 0$. In such cases, each point in coordinate space (the $x-y$ plane) may be transformed into a line in the parameter space (the $\rho-\phi_{0}$ plane). Thus the hits from a given track will be associated with a set of lines in parameter space with a vertex corresponding to the track's direction and curvature.

When the paramter space is discretized in the form of a histogram, the line for each hit becomes a set of bins. The HTF fills these bins, and the vertices are identified as local maxima in the histogram. These vertices are used to define a set of track candidates that are passed, along with the candidates from the AA scheme, to the selection procedure.

\subsubsection{Track Selection}

The pool of hypotheses established by the AA and HTF schemes are filtered by an initial set of requirements that depend on the number and types of missed hits. Three classes of misses are defined:

Inside Misses: Missed hits which that occur between the innermost and outermost hits on the track.

Forward Misses: Missed hits after the outermost hit on the track.

Backward Misses: Missed hits before the innermost hit on the track.

Collectively, forward and backward misses are referred to as outside misses.

To pass the selection, a track must satisfy these initial criteria: 

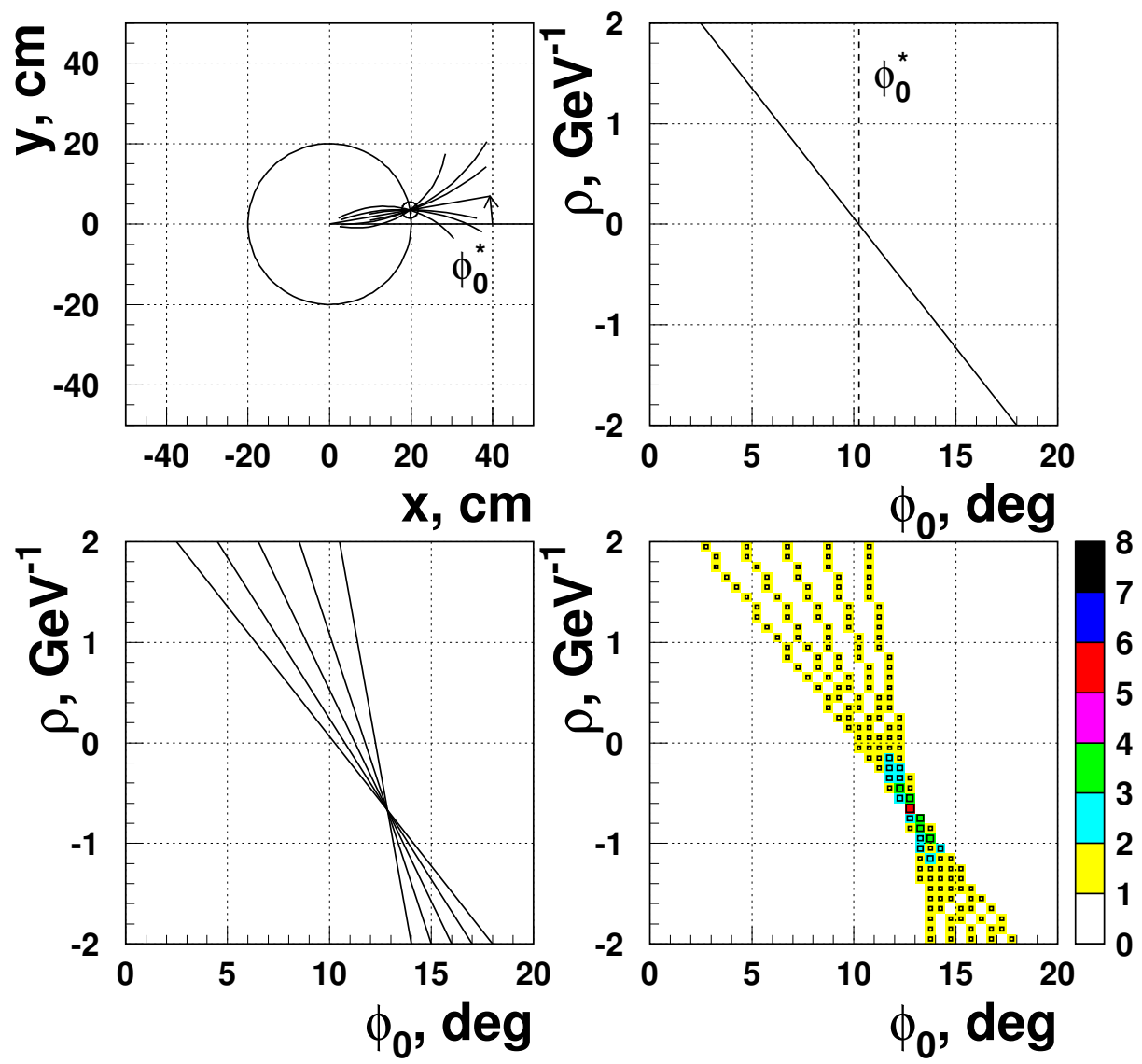

Figure 3.1: Example of track reconstruction with HTF. The top left shows the family of tracks that contain a given hit. The top right shows the representation of these tracks in terms of the curvature $(\rho)$ and azimuthal angle $\left(\phi_{0}\right)$. In the bottom left plot the intersection of the lines for each hit on a track indicate the parameter values for that track. In the bottom right, the lines are used to fill a histogram and the intersection is identified as a local maximum [26]. 
- The track must have at least four hits, with hits in both the axial and stereo layers of the complete tracking system.

- There may not be more than three inside misses.

- The total number of outside misses may not be greater than six.

- The track may have, at most, two inside misses in the SMT.

- The total number of hits must be at least five times the number of misses.

- For tracks with inside misses:

- The total of the inside and forward misses must be less than five.

- The total of the inside and backward misses must not be greater than three.

The remaining track candidates are sorted by the number of hits (in decreasing order), the number of misses (in increasing order) and the fit $\chi^{2}$ (in increasing order). Hypotheses with axial hits that are not shared with entries earlier in the list are accepted:

- $N_{\text {shared }} \leq \frac{2}{3} N_{\text {tot }}$.

- $N_{\text {shared }} \leq N_{\text {tot }} / 5$ and $N_{\text {tot }}-N_{\text {shared }}>3$.

Here, $N_{\text {tot }}$ is the total number of axial hits on the track, while $N_{\text {shared }}$ is the number of such hits that are in common with previous track candidates. This process removes ambiguous solutions for a given trajectory.

The remaining tracks are grouped together into vertices by an algorithm similar to the one described in Section 3.2. Vertices are required to have at least five tracks with $\chi^{2}<36$. The track candidates are re-sorted and filtered as before, except that those consistent with one of the vertices are assumed to have two additional (unshared) hits. The track ordering controls which hits are considered shared; therefore previously accepted hypotheses may be rejected, with a preference for keeping candidates that are associated with a vertex.

\subsection{Vertex Reconstruction}

The vertex reconstruction algorithm [27] locates the collisions that occur in each beam-crossing and selects from them the hard-scatter interaction that 


\begin{tabular}{|c|c|}
\hline Track variable & Requirement \\
\hline$p_{T}$ & $\geq 0.5 \mathrm{GeV} / c$ \\
SMT hits & $\geq 2$ \\
$\frac{D C A}{\sigma_{D C A}}$ & $\leq 100$ \\
\hline
\end{tabular}

Table 3.1: Selection criteria for tracks in the first pass of the vertex reconstruction.

was responsible for triggering the event. Although events with several grazing $p \bar{p}$, minimum bias, collisions are common, the likelihood that more than one collision per beam-crossing will merit further study is negligible. The vertex finding is performed in two passes. In the first pass, candidates are built from tracks that satisfy loose selection requirements. The second pass tightens the track selection and re-fits the vertices by using the remaining tracks. The hardscatter (primary vertex) is then chosen with a probabilistic method based on the associated track momenta.

The loose track requirements imposed during the first pass vertexing are described in Table 3.1. The SMT hit requirement is not applied for simulated data. At this stage, the distance of closest approach (DCA) and its uncertainty $\left(\sigma_{D C A}\right)$ are measured with respect to the detector origin.

The selected tracks are fit to a common vertex position with the Kalman filter algorithm described in Section 3.2.1. If the resulting $\chi^{2}$ is greater than 10, then the track with the largest contribution is removed. This process is repeated until the vertex $\chi^{2}$ is less than 10 . The algorithm then iterates over the excluded tracks to identify additional vertices. In this way, a preliminary list of vertices is generated.

In the second pass of the algorithm, the nominal vertex positions are used to determine the location of the beam spot. The first stage algorithm is applied again, except that the input tracks are required to have $D C A / \sigma_{D C A}<3$, now measured with respect to the beam position.

The vertex associated with the hard-scatter is separated from the grazing collisions by using a likelihood value that is formed from the associated track momenta [28]. For each track associated with a vertex, a probability $P\left(p_{T}\right)$ is assigned:

$$
P\left(p_{T}\right)=\frac{\int_{\log _{10}\left(p_{T}\right)}^{\infty} F(x) d x}{\int_{\log _{10}(0.5)}^{\infty} F(x) d x}
$$

where $F(x)$ is the distribution of $x=\log _{10}\left(p_{T}\right)$ for tracks from simulated 


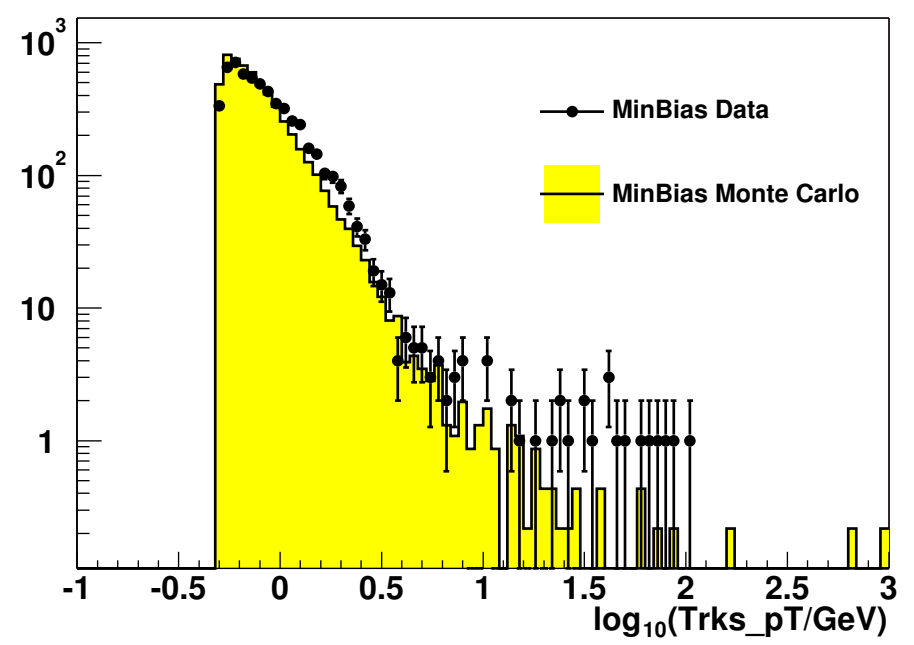

Figure 3.2: Distribution of $\log _{10}\left(p_{T}\right)$ for tracks from minimum bias events. The collider data distribution (points) is taken from a $Z \rightarrow \mu \mu$ sample. The solid histogram shows the distribution for simulated events [27].

minimum bias events, and is shown in Figure 3.2. The product of all associated tracks is calculated and weighted by the track multiplicity to form a vertex minimum bias probability that is independent of the number of associated tracks. The vertex with the lowest such probability is selected as the primary vertex.

\subsubsection{Vertex Fitting}

The goal of the Kalman filter is to identify the vertex position $\vec{x}=(x, y, z)$ and the momentum $\vec{q}_{k}$ of every track $k$ associated with it. If $\mathbf{m}_{k}$ is the vector of parameters for that track (the track $\mathrm{z}, \phi, \cot (\theta)$, impact parameter and curvature), then a measurement vector and its covariance (error) matrix may be defined:

$$
\begin{array}{r}
\vec{d}^{m}=\left(\begin{array}{c}
\vec{m}_{1} \\
\vec{m}_{2}
\end{array}\right) \\
V=\left(\begin{array}{cc}
V_{1} & 0 \\
0 & V_{2}
\end{array}\right)
\end{array}
$$

where $V_{k}$ is the error matrix for a given track. Furthermore, the vector of 
vertex parameters is defined as:

$$
\vec{q}=\left(\begin{array}{c}
\vec{x} \\
\overrightarrow{q_{1}} \\
\overrightarrow{q_{2}}
\end{array}\right)
$$

Finally the function, $\vec{d}(\vec{q})$, defines the track parameters for a given $\vec{q}$. An iterative procedure described in reference [27] is used to minimize:

$$
\chi^{2}=\left(\overrightarrow{d^{m}}-\vec{d}(\vec{q})\right)^{T} V^{-1}\left(\overrightarrow{d^{m}}-\vec{d}(\vec{q})\right)
$$

and find the vertex fit parameters.

\subsection{Jet Reconstruction}

The objective of jet reconstruction is to measure the momenta of the quarks and gluons produced in a collision. Since hadronization processes cause these partons to evolve into sprays of secondary particles (i.e., the jets) before any interactions with the detector, this task presents special challenges.

The necessity of comparison to theoretical predictions demands a jet reconstruction algorithm must yield the same results using hard-scatter partons, final state particles, or detector elements. Furthermore, the procedure should be insensitive to infrared and collinear effects, as shown in Figures 3.3 - 3.5: the reconstructed jets should not change with the addition of soft radiation (i.e., low energy deposits in the detector) or the splitting of energy between collinear particles (or adjacent detector elements) [29]. These problems arise from the use of detector elements (or particles) over some threshold as seeds to define the initial jet candidates (called proto-jets). Seedless algorithms exist that avoid these difficulties; however, they are too computationally intensive for practical use in hadron collider environments.

The jet reconstruction procedure searches the calorimeter for cones of radius $R_{\text {cone }}=0.5$ in Y- $\phi$ space. Cells with energies greater than some threshold are built into towers that are sorted by $p_{T}$. These towers are pre-clustered into narrow cones that are used as seeds to find an initial list of proto-jets. The midpoints between these jet candidates define a second set of seeds for the reconstruction of additional proto-jets. A splitting and merging algorithm removes any shared towers, then quality criteria are applied to form the final jets.

The first step in the reconstruction algorithm is to remove any calorimeter cells that exhibit noisy behavior from the list of inputs. The initial list 

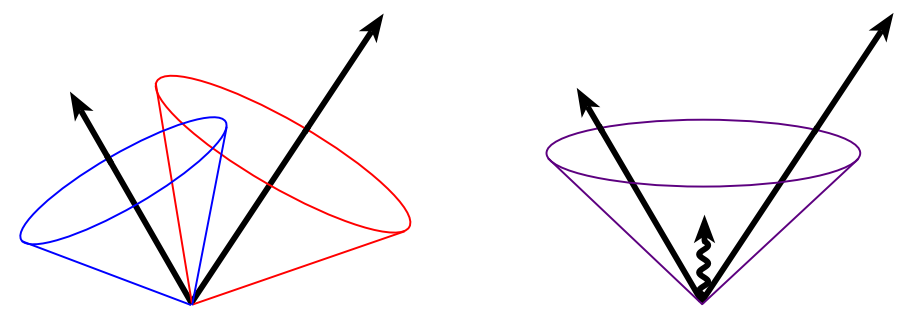

Figure 3.3: Example of infrared sensitivity to soft gluon radiation. On the left, two partons are reconstructed into two jets. On the right, an additional low energy gluon becomes a seed for the reconstruction algorithm that clusters both high energy partons into a single jet.

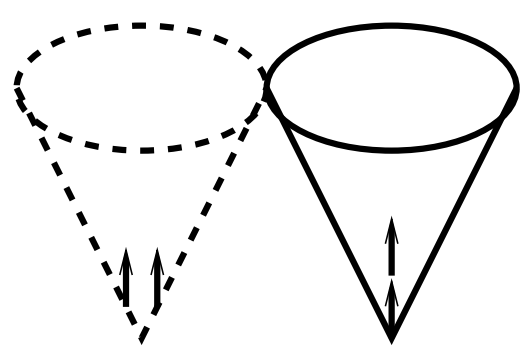

Figure 3.4: Illustration of collinear sensitivity. On the left, no jet is reconstructed because the energy is distributed between two collinear partons (or adjacent detector elements), both below the seed threshold. On the right, the energy is more narrowly distributed and the reconstruction succeeds.

consists only of cells with an energy greater than 2.5 times the width of that cell's pedestal distribution, $\sigma_{\text {ped }}[30]$. The new anomolous deposit algorithm (NADA) calculates the total energy contained in the neighboring cells, excluding those below some threshold, $E_{\text {cell }}^{\text {cut }}[31]$. If the total is less than another threshold, $E_{\text {cube }}^{\text {cut }}$, the cell is identified as noisy and discarded. The values of $E_{\text {cell }}^{\text {cut }}$ and $E_{\text {cube }}^{\text {cut }}$ depend on the cell energy as specified in Reference [32]. Cells with an energy less than $4 \sigma_{\text {ped }}$ are also ignored, unless there is an adjacent cell with an energy greater than $4 \sigma_{\text {ped }}$ [33] [34].

A four-momentum is calculated for every calorimeter tower containing cells that survived the noise suppression algorithms. This is accomplished by summing the four-momenta of every cell $i$ in the tower, with:

$$
\begin{aligned}
E^{i} & =\text { cell energy } \\
p_{x}^{i} & =\left(E^{i} / c\right) \sin \theta^{i} \cos \phi^{i}
\end{aligned}
$$



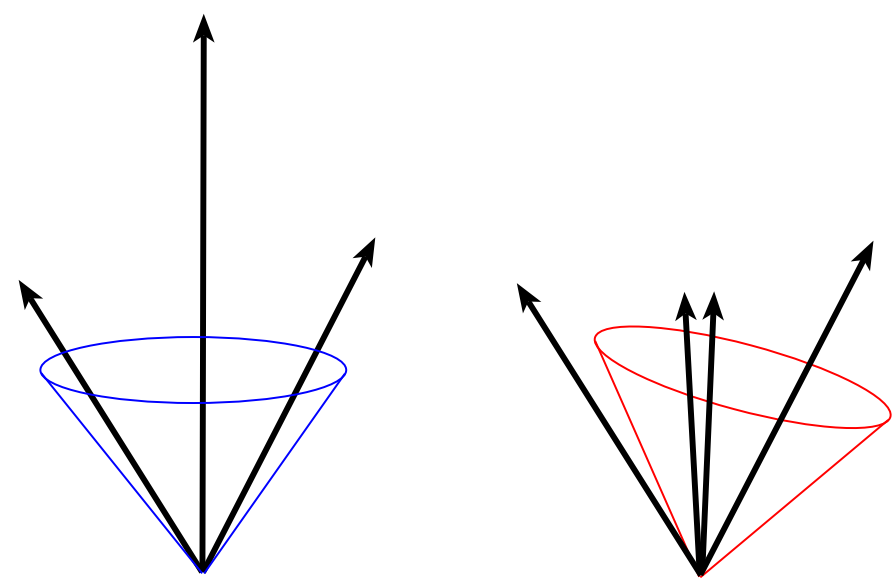

Figure 3.5: Example of another collinear problem. The reconstruction processes particles in order of $p_{T}$, removing them from the list of seeds as they are added to jets. On the right, the energy of the central parton from the left is split. Therefore, the initial seed changes, resulting in a decrease of the total energy clustered into the jet.

$$
\begin{aligned}
& p_{y}^{i}=\left(E^{i} / c\right) \sin \theta^{i} \sin \phi^{i} \\
& p_{z}^{i}=\left(E^{i} / c\right) \cos \theta^{i}
\end{aligned}
$$

where $\left(\theta^{i}, \phi^{i}\right)$ indicate the angular position of the cell with respect to the primary vertex. If the most energetic cell in a tower is within the coarse hadronic layer or near the uninstrumented boundaries between the central and end calorimeters, then it is not counted in this summation, as such cells are especially susceptible to noise.

Pre-clusters are built from seed towers with $p_{T}>0.5 \mathrm{GeV} / c$, in order of decreasing transverse momentum. A tower is added to a pre-cluster if it has $p_{T}>1 \mathrm{MeV} / c$ and is closer than 0.3 , in $\eta-\phi$ space, to the pre-cluster. A tower added to a pre-cluster is removed from the list so that it can belong at most one such object. Only pre-clusters with at least two towers and a total $p_{T}>1 \mathrm{GeV} / c$ are accepted.

The pre-clusters are examined in order of decreasing $p_{T}$. If the distance $\Delta R=\sqrt{\Delta Y^{2}+\Delta \phi^{2}}$ to the nearest proto-jet is greater than $0.25\left(=R_{\text {cone }} / 2\right)$, then the pre-cluster is used as a seed. Proto-jets are built from seeds by defining a cone of radius $\Delta R=R_{\text {cone }}$ around the seed center. All active towers within the cone are added to the proto-jet. Their four-momenta are summed to give a total for the proto-jet; this also makes possible the calculation of the angular coordinates $Y$ and $\phi$ of the jet. If the proto-jet has a $p_{T}$ that is less 
than half of the jet reconstruction threshold $(8 \mathrm{GeV} / c)$, then it is discarded. Otherwise, a new cone centered on the proto-jet $Y$ and $\phi$ is defined, which replaces the previous proto-jet. This process is repeated at most fifty times until the $\Delta R$ between successive iterations is less than 0.001 .

At this stage, the use of seeds causes considerable sensitivity to the infrared and collinear effects already discussed. However, it has been shown that the use of midpoints between proto-jets as an additional set of seeds suppresses these issues [29]. There is still some inefficiency for reconstructing jets with split seeds, as shown in Figure 3.4, but this is negligible for jets with $p_{T}>20 \mathrm{GeV} / c$. Therefore the midpoint between every pair of proto-jets that $R_{\text {cone }}<\Delta R<$ $2 R_{\text {cone }}$ is determined by summing their momenta. An additional set of protojets is reconstructed, using the midpoints as seeds.

The resulting jet candidates often share calorimeter towers. Overlapping jets are merged or split to avoid double counting the energy in such towers. The list of proto-jets is sorted, in order of decreasing transverse momentum. Each element in the list is examined for overlapping proto-jets; the highest $p_{T}$ neighbor (if any) sharing energy with the first jet is considered. If the total transverse momentum of the shared towers is greater than half the $p_{T}$ of the second jet, then the two are merged. Otherwise, the jets are split, and each shared tower is assigned to the closest proto-jet in $Y-\phi$ space. The jet fourmomenta are recalculated and the list is reordered. This process is repeated until there are no overlaps. Jets with $p_{T}<8 \mathrm{GeV} / c$ are rejected.

After the jet reconstruction is completed, a final set of quality criteria are applied:

- The fraction of the jet energy deposited in the electromagnetic layers of the calorimeter must be between 0.05 and 0.95 .

- The fraction of the jet energy deposited in the coarse hadronic layer of the calorimeter must be less than 0.4.

- The ratio of the energies in the two most energetic cells in the jet should be less than 10 .

- No single calorimeter tower may contain more than $90 \%$ of the jet energy.

- The ratio of the total energy in Level 1 trigger towers associated with the jet to the reconstructed energy is calculated (energy in the coarse hadronic layer of the calorimeter is not counted). This ratio must be at least 0.4 (or 0.2 in the inter-cryostat region). 
Distributions of these jet quality variables are shown for real and fake (noise) jets in Figures 3.6 and 3.7. To obtain a control sample of good jets, events with missing transverse energy less than $5 \mathrm{GeV}$ are selected. The jet under examination must have a $\phi$-separation of at least 2.9 from a photon or a jet that satisfies the above quality requirements. Two categories of fake jets are considered: the first type consists of an extra jet in two jet or photon plus jet events with missing $E_{T}>10 \mathrm{GeV}$. The $p_{T}$ difference between the two objects is required to be less than $5 \mathrm{GeV} / c$. The second sample consists of events with at least six reconstructed jets and $H_{T}>20 \mathrm{GeV}$. In both cases, fake jets must have no tracks with $p_{T}>0.5 \mathrm{GeV}$ and $\Delta R<0.5$ [35].

\subsubsection{Jet Energy Scale}

Several effects can cause the initial measured energy of a jet, calculated as a summation over its constituent cells, to differ from the original particle kinematics. The calorimeter has different repsonses for the various species of particles that typically compose a jet (dominantly protons, neutrons, photons, pions and kaons). Moreover, energy is lost in the cracks between calorimeter modules. These effects are modeled by the response function $\mathcal{R}_{\text {jet }}$.

The jet cone will not always encompass all of the consitutent particles. Furthermore, some particles that are initially contained within the cone may bend out of it, because of the magnetic field in the tracker, or induce showers in the calorimeter outside of the cone. The fraction of energy included in the cone, $\mathcal{R}_{\text {cone }}$, parameterizes these out-of-cone corrections.

Finally, the jet energy is increased by some offset, $E_{0}$, that arises from electronics noise, the decay of the uranium absorbers, additional low energy collision, low energy debris from the hard-scatter under study (i.e., the underlying event) and accumulated energy from previous events.

The jet energy scale adjusts the initial measurement, $E_{\text {raw }}$, for all of these corrections. Reference [36] describes the correction procedure in detail. The energy of the original particle jet, $E_{\text {particle }}$, is given by:

$$
E_{\text {particle }}=\frac{E_{\text {raw }}-E_{0}(\eta)}{\mathcal{R}_{\text {jet }}\left(E_{\text {raw }}, \eta\right) \mathcal{R}_{\text {cone }}\left(E_{\text {raw }}, \eta\right)}
$$

where $\mathcal{R}_{\text {jet }}$ and $\mathcal{R}_{\text {cone }}$ are functions of the jet pseudorapidity and energy. The offset energy also depends on the jet $\eta$ but is independent of its energy. In principle, all of these corrections vary with luminosity. This dependence is not currently modeled, but, instead, it is included in the systematic uncertainty on the jet energy scale. 

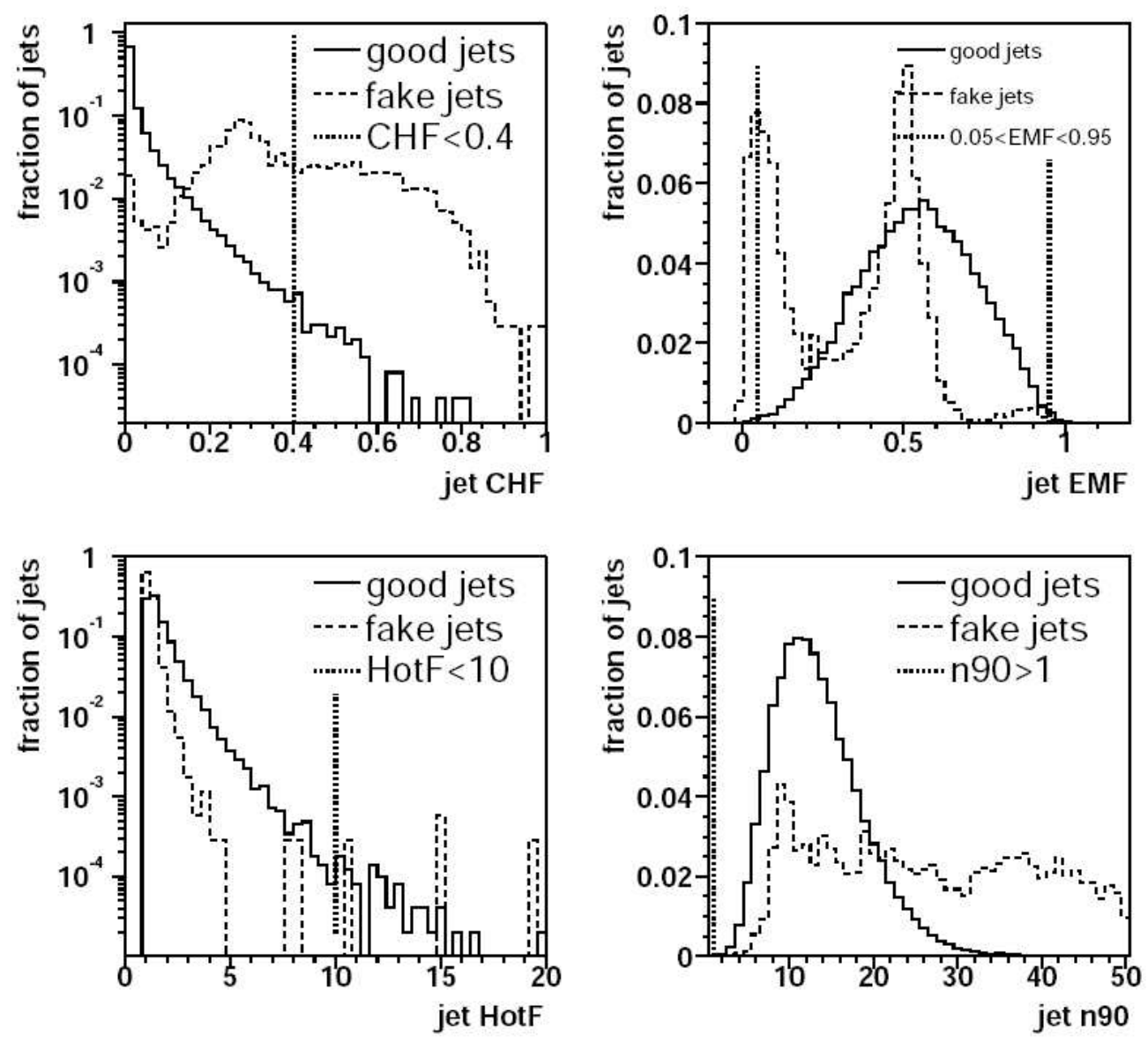

Figure 3.6: Distributions of jet quality variables for both good and fake jets in the central calorimeter. The upper left plot shows the fraction of the jet energy contained in the coarse hadronic layers. The top right plot shows the energy fraction in the electromagnetic portion of the calorimeter. The lower left histogram shows the energy ratio of the two most energetic cells in the jet. The bottom right graph shows the number of calorimeter towers that contain at least $90 \%$ of the jet energy [35]. 


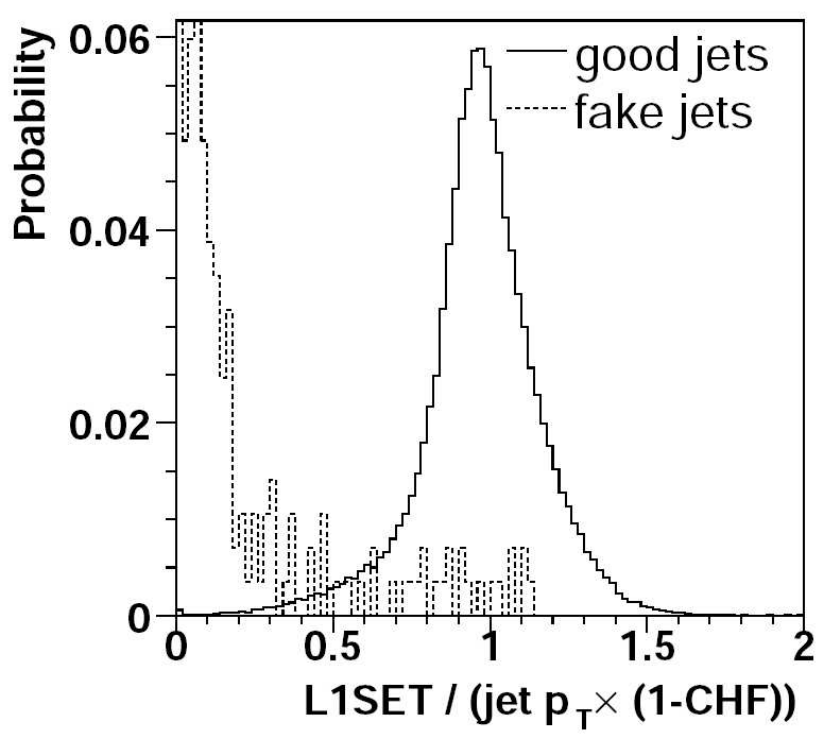

Figure 3.7: Distribution of the ratio of the Level 1 trigger energy in a jet to the transverse momentum after removing the coarse hadronic energy [35].

The offset correction is derived by measuring the calorimeter energy density for events that have satsified a minimum bias trigger (i.e., hits in the luminosity counters mounted on the calorimeter endcaps). The response correction, $\mathcal{R}_{\text {jet }}$, is measured in events with a photon opposite a jet in $\phi$. The calorimeter response to electrons and photons may be measured from mass resonances, such as the $Z$ and the $J / \psi$. After applying this calibration, the missing $E_{T}$ in $\gamma$-jet events is used to determine $\mathcal{R}_{\text {jet }}$ : since $\gamma$-jet events rarely contain high energy neutrinos, any apparent momentum imbalance is dominated by mismeasurement of the jet energy. The out-of-cone correction is derived by examining the decrease in the energy density oustide of the cone of a reconstructed jet.

These correction factors will differ between the collider and simulated data samples because of imperfect detector modelling in the simulation. Therefore, the calibration process is repeated using simulated samples that correspond to the data sets that are used to determine the jet energy scale on collider data. The final correction factors for real and simulated events are shown in Figures 3.8 and 3.9, respectively. 

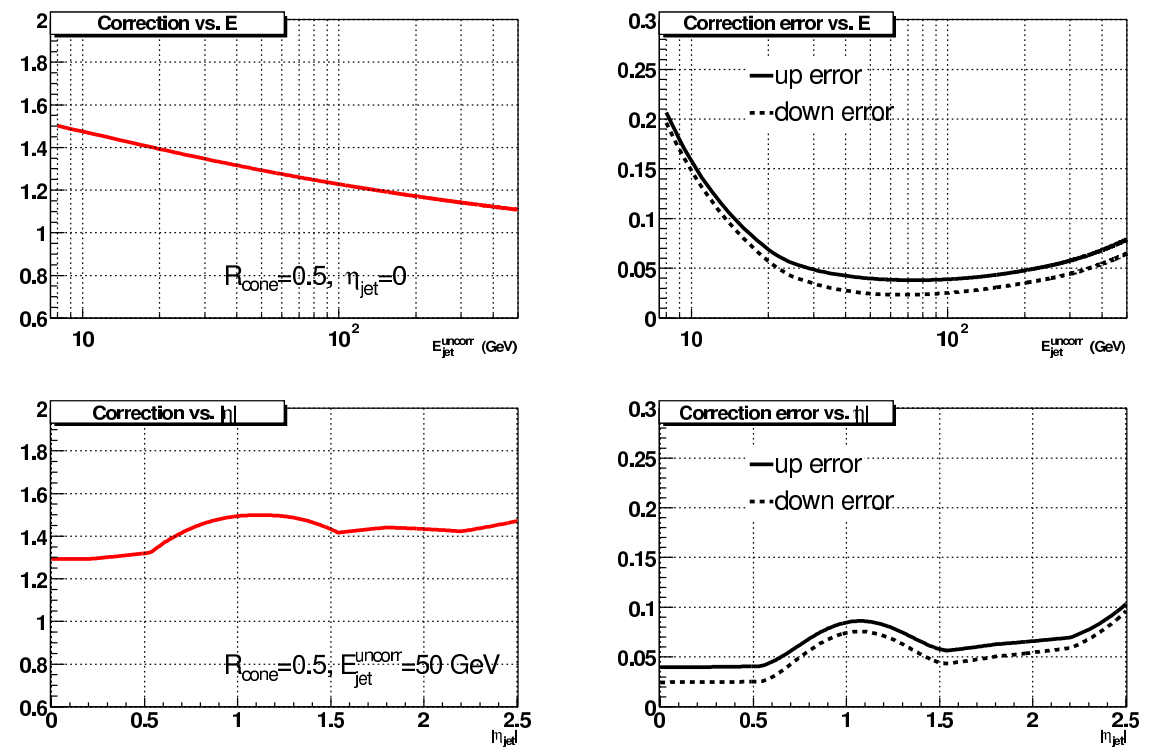

Figure 3.8: Jet energy scale correction factor (left) and uncertainty (right) for collider data as a function of jet energy (top) and pseudorapidity (bottom) [36]. 

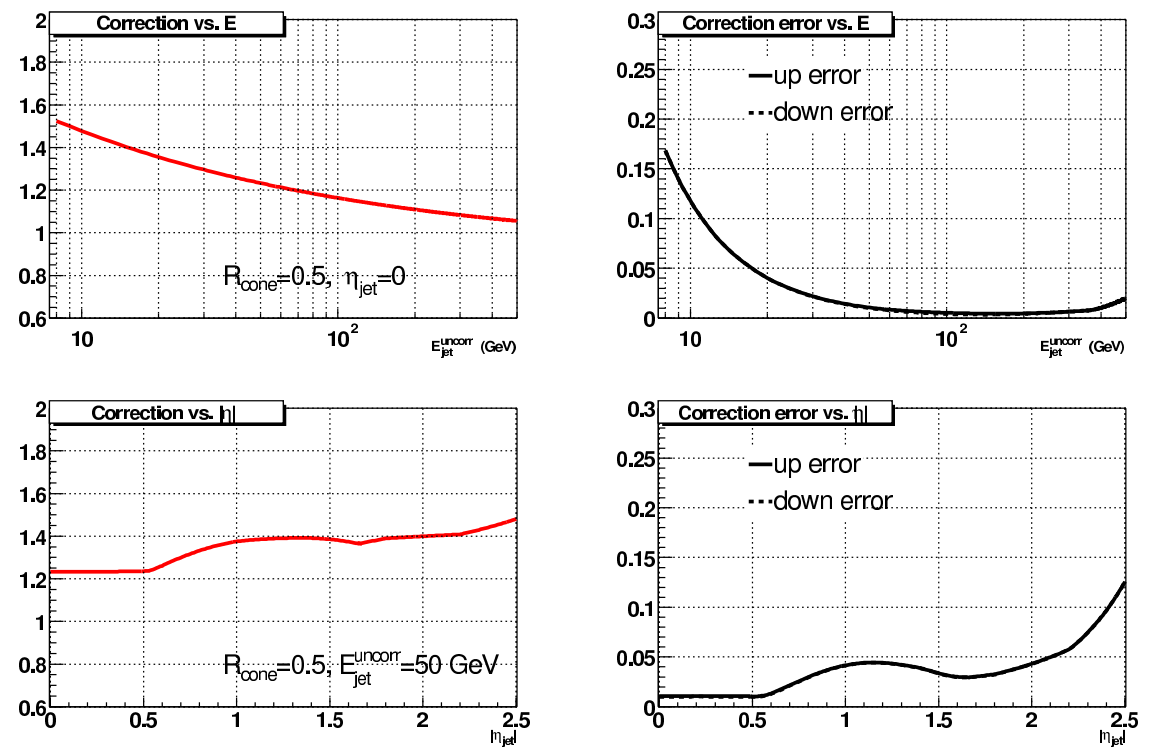

Figure 3.9: Jet energy scale correction factor (left) and uncertainty (right) for simulated events as a function of jet energy (top) and pseudorapidity (bottom) [36]. 


\subsection{Muon Reconstruction}

Muons are reconstructed by independently associating hits in the drift chambers into segments in each of the (A, B and C) muon system layers. Hits in the scintillator paddles are added to the segments [37]. Segments that are consistent with a common trajectory are merged into tracks. These local muons are then combined with the central tracks identified in the SMT and the CFT to form global muons. A final set of requirements is imposed to supress any remaining backgrounds.

The segment reconstruction begins by considering only PDT and MDT hits that are consistent in time with a beam crossing. This criterion rejects backgrounds from the radioactive environment and (anti-) protons scattering off of the beam pipe or the Tevatron magnets. The hits are mapped into a coordinate space that is oriented with the $z$-axis parallel to the drift chamber wires, which are contained in the $y-z$-plane. The timing of a hit, as shown in Figure 3.10, constrains its location to a circle surrounding the wire. All possible pairs of hits within a chamber are formed, provided they are not on the same drift circle and are separated by less than $20 \mathrm{~cm}$ in the $y$-direction. Additionally the two hits may not be in the same plane of wires unless they are consistent with a track passing between adajcent wires (for examples, see Figure 3.10).

Pairs are combined into segments of three or more hits and fit to a straight line. Every permutation is allowed, including the combination of multiple pairs into a single segment. The segments are sorted, first by the number of hits and then by the fit $\chi^{2}$; only the first four segments are used. Nearby scintillator hits in the same layer are associated with the segment. Since each segment is wholly contained within one layer of the muon system, the segments are matched and re-fit again into local tracks [38]; although the fit is not required to converge. The toroidal magnet provides a local $p_{T}$ measurement for muons with segments in both the $\mathrm{A}$ and $\mathrm{B}$ or $\mathrm{C}$ layers. If the fit does not converge, then an estimate of the muon $p_{T}$ is obtained by using the bending angle between segments. The resolution of the transverse momentum measurement is limited at low energies $(\sim 6 \mathrm{GeV})$ by multiple scattering in the iron of the magnet, and at high energies $(\sim 10 \mathrm{GeV})$ by the spatial resolution of the drift chambers and scintillators [39].

If the local fit converges, the resulting measurements of the muon momentum and the A layer position are transformed into the five standard tracking parameters (see Section 3.2.1) and propagated back to the point of closest approach with respect to the primary vertex. Central tracks with $p_{T}>1 \mathrm{GeV} / c$, and angular separations $\Delta \phi<1$ and $\Delta \theta<1$ are considered for matching 


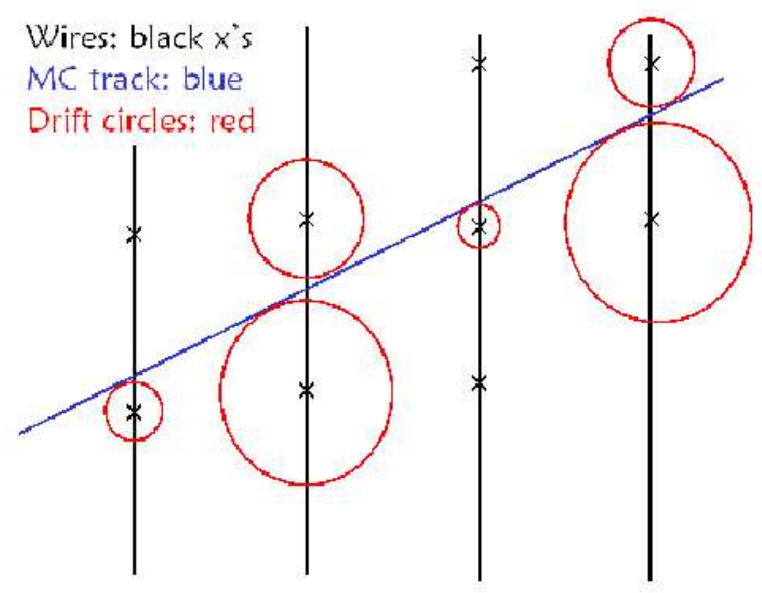

Figure 3.10: Example of a muon traversing a drift chamber. Hits are constrained by their timing information to lie on circles (shown in red) around the wires with signals.

to the muon. A new set of parameters, $P_{f}$, is calculated as an average of the central track and local muon parameters, $P_{t r k}$ and $P_{l o c}$, weighted by their uncertainties:

$$
P_{f}=\left(E_{\text {trk }}^{-1}+E_{l o c}^{-1}\right)^{-1}\left(E_{t r k}^{-1} P_{t r k}+E_{l o c}^{-1} P_{l o c}\right)
$$

where $E_{t r k}$ and $E_{l o c}$ are the central and local track covariance matrices. The central track with the best combination:

$$
\chi^{2}=\left(P_{t r k}-P_{l o c}\right)^{T}\left(E_{t r k}+E_{l o c}\right)^{-1}\left(P_{t r k}-P_{l o c}\right)
$$

is selected as the match [40]. When the local muon fit does not converge, tracks are propagated to the A layer and the closest in $\theta-\phi$ space is chosen, provided it satisfies the pre-selection criteria used for the $\chi^{2}$ matching. In this dissertation, the muon kinematic variables are those of the central track, even if the local fit converges.

A muon thus found is assigned a category, nseg, depending on whether it contains $\mathrm{A}, \mathrm{B}$ or $\mathrm{C}$ layer segments and the presence of a matching track as shown in Table 3.2 [41]. From this stage on, the B and C layers are always considered together. The quality is also defined as being either tight, medium or loose based on the muon nseg and the number of hits in the muon system [42].

Tight muons must meet the following criteria: 


\begin{tabular}{|c|c|c|}
\hline$n s e g$ & Segment requirements & Track match \\
\hline+3 & A and BC layers & yes \\
+2 & BC layers & yes \\
+1 & A layer & yes \\
-1 & A layer & no \\
-2 & BC layers & no \\
-3 & A and BC layers & no \\
\hline
\end{tabular}

Table 3.2: Definition of the reconstructed muon categories, nseg. Categories are defined exclusively so that (for example) an $n s e g=+3$ muon is considered to satisfy neither $n s e g=+1$ or $n s e g=-3$.

- $|n s e g|=3$.

- The local fit must converge.

- At least two hits in the A layer drift chambers (wire hits).

- At least one A layer scintillator hit.

- At least three wire hits in the BC layers.

- At least one hit in the BC layer scintillators.

The requirements for loose and medium muons vary according to the value of nseg. Medium muons with $\mid$ nseg $\mid=3$ must satisfy:

- At least two hits in the A layer drift chambers.

- At least one A layer scintillator hit.

- At least two wire hits in the BC layers .

- At least one hit in the BC layer scintillators is required, except for muons in the central muon system with less than four BC PDT hits.

An $\mid$ nseg $\mid=3$ loose muon is permitted to fail any one of the medium requirements just listed. Muons with $n s e g=-1$ and $n s e g=-2$ are rejected (i.e., they are required to have a track match). Those with $n s e g=+1,+2$ are loose provided meet the following criteria:

- $n \operatorname{seg}=2$ : 
- At least one BC layer scintillator hit.

- At least two BC layer wire hits.

- $n \operatorname{seg}=1$ :

- At least one A layer scintillator hit.

- At least two A layer wire hits.

Such muons are considered medium if they are also in the bottom of the central muon system, where there is reduced coverage. The probability for low momentum muons to penetrate the toroid may be estimated from simulations; if this probablility is sufficiently small, an $n s e g=1$ loose muon is promoted to medium quality [42].

Only medium muons with a central track satisfying the follwing criteria are used in this analysis:

- To reject muons arising from cosmic rays, the average time of the $\mathrm{A}$ layer scintillator hits, $t_{A}$, and the BC layer scintillator hits, $t_{B C}$, must be within $10 \mathrm{~ns}$ and $15 \mathrm{~ns}$ of the beam crossing, respectively.

- The muonic decays of low energy kaons and other hadrons will cause a kink in the central track, inducing a mismeasurement of the track momentum. To supress the background from such in-flight decays to apparently high- $p_{T}$ muons, the local momentum should be at least 8 $\mathrm{GeV} / c$.

- The central track should be well measured and consistent with the primary vertex. This requirement further suppresses the cosmic and inflight decay backgrounds. In particular, the track should:

- have at least 14 CFT and 4 SMT hits,

- have a $\chi^{2}$ per degree of freedom less than 4 ,

- pass within $1 \mathrm{~cm}$ in $\mathrm{z}$ and $0.02 \mathrm{~cm}$ in the $\mathrm{x}-\mathrm{y}$ plane of the primary vertex.

- The muon should have a transverse momentum of at least $4 \mathrm{GeV} / c$, as measured from the central track.

The backgrounds to muons from the decay of $W$ and $Z$ bosons are primarily associated with hadronic activity near the reconstructed muon. Conversely, any lepton from an electroweak boson decay is typically well separated from any other particle in the event. Therefore, an isolation requirement is a powerful method to identify such events. A muon is considered isolated if: 
- It is separated by a distance $\Delta R=\sqrt{\Delta \eta^{2}+\Delta \phi^{2}}>0.5$ from the nearest jet.

- The energy deposited in the calorimeter inside of a 0.4 cone but outside of a 0.1 cone of $\eta-\phi$ around the muon is less than $2.5 \mathrm{GeV}$.

\subsection{Missing $E_{T}$ Calculation}

Neutrinos only participate in the weak and gravitational forces; thus, they do not interact with the detector and cannot be directly observed. However, it is possible to infer the presence of a high energy neutrino from the observation of a significant transverse momentum imbalance in a collision. The missing transverse energy (missing $E_{T}$ or $\mathbb{E}_{T}$ ) is defined as the negative of the vector sum of all the transverse energy in the event. The summation is performed over calorimeter cells surviving the hot-cell supression described in Section 3.3, neglecting cells in the coarse hadronic layers, unless they are contained in good reconstructed jets [33]. The momentum of good, though not neccessarily isolated, muons is also included.

$$
\begin{aligned}
& E_{x}=-\sum_{\text {cells } \neq C H} E_{i} \sin \theta_{i} \cos \phi_{i}-\sum_{\text {jets }} c p_{x}^{\text {jet }} f_{C H}^{j e t}-\sum_{\text {muons }} c p_{x}^{\mu} \\
& \mathscr{E}_{y}=-\sum_{\text {cells } \neq C H} E_{i} \sin \theta_{i} \sin \phi_{i}-\sum_{\text {jets }} c p_{y}^{\text {jet }} f_{C H}^{j e t}-\sum_{\text {muons }} c p_{y}^{\mu} \\
& \mathscr{E}_{T}=\left|\vec{E}_{T}\right|=\sqrt{\mathscr{E}_{x}^{2}+\mathbb{E}_{y}^{2}}
\end{aligned}
$$

where $E_{i}, \theta_{i}$ and $\phi_{i}$ are the energy and angular coordinates, with respect to the primary vertex, of the $i^{t h}$ cell. The $x$ and $y$ components of a reconstructed jet or muon momentum are given by $p_{x}^{\text {jet }}$ and $p_{y}^{j e t}$ or $p_{x}^{\mu}$ and $p_{y}^{\mu}$, respectively. The fraction of the energy of a jet in the $\mathrm{CH}$ layers is $f_{C H}^{j e t}$. Although the jet energy scale is not applied to the $\mathbb{E}_{T}$, it is corrected for the energy scale variation between real and simulated data. In Monte Carlo events:

$$
\begin{aligned}
& \mathbb{E}_{x}{ }^{\mathrm{corr}}=\mathbb{E}_{x}-\sum_{j e t s} p_{x}^{\text {raw }}\left(\frac{C_{\text {data }}}{C_{m c}}-1\right) \\
& \mathbb{E}_{y}{ }^{\mathrm{corr}}=\mathbb{E}_{y}-\sum_{j e t s} p_{y}^{\text {raw }}\left(\frac{C_{d a t a}}{C_{m c}}-1\right)
\end{aligned}
$$



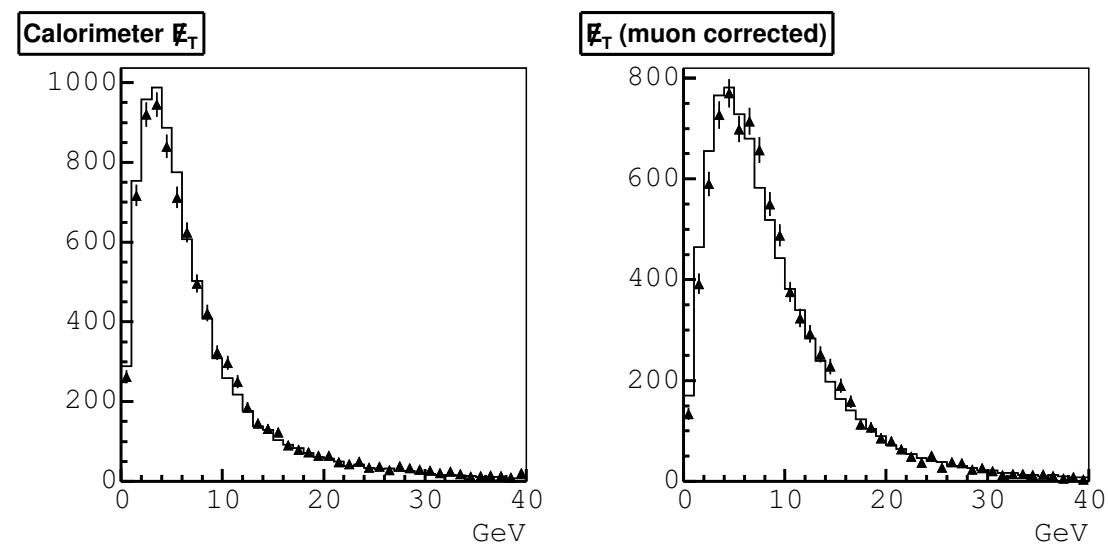

Figure 3.11: The missing transverse energy in $Z \rightarrow \mu \mu$ events before (left) and after (right) the correction for muon momenta. The points indicate collider data while the solid line shows simulated events.

where $\left(p_{x}^{\text {raw }}, p_{y}^{\text {raw }}\right)$ represent the uncorrected transverse momentum of a jet and $C_{\text {data }}\left(C_{m c}\right)$ is the energy scale correction factor for a jet from real (simulated) data. The missing $E_{T}$ from $Z \rightarrow \mu \mu$ events is shown in Figure 3.11.

\subsection{Electron and Photon Reconstruction}

Electrons ${ }^{1}$ and photons, collectively referred to as electromagnetic particles exhibit similar behavior in the calorimeter: they deposit almost all of their energy in a narrow shower in the electromagnetic layers. Electrons have only a minor impact on the analysis presented in this dissertation, and photons are not used at all; thus, their reconstruction is only described briefly.

The electromagnetic reconstruction process is similar to the pre-clustering algorithm used in the first step of the jet reconstruction. Seed towers of 500 $\mathrm{MeV}$ are used to identify cones with a radius of 0.4 in $\eta-p h i$ space. The calorimeter shower is required to be narrow and mostly contained in the electromagnetic layers. A shower shape $\chi^{2}$ (the H-matrix) is used to control backrounds from jets [43]. An isolation fraction, $f_{i s o}$, is defined in terms of

\footnotetext{
${ }^{1}$ For the purposes of this dissertation the distinction between particles and antiparticles is unimportant. Unless explicitly noted, whenever a particle is discussed, the charge conjugate equivalent is also implied. Thus positrons are also included in this categorization.
} 
the electromagnetic energy contained in cones of 0.2 and 0.4 in $\eta-\phi$ space, referred to as $E(0.2)$ and $E(0.4)$, around the electron axis:

$$
f_{\text {iso }} \equiv \frac{E(0.4)-E(0.2)}{E(0.2)}
$$

Since photons do not generally leave any signatures in the tracking system, the presence of a track match distinguishes electrons from photons. A likelihood based on the matched track as well as any additional nearby tracks provides additional suppression of hadronic backgrounds and improves the electronphoton distinction [44].

Electrons are required to satisfy:

- The H-matrix $\chi^{2}$ should be less than 75 .

- When the H-matrix $\chi^{2}$ is calculated without use of the shower shape along the $z$-axis, it should be less than thirty.

- The isolation fraction $f_{\text {iso }}$ should be less than 0.15 .

- The energy deposited in the electromagnetic layers of the calorimeter should be at least $90 \%$ of the total.

- The electron must be matched to a central track. 


\section{Chapter 4}

\section{Event Simulation}

Detailed event simulations are often used to compare the experimental data collected at the Tevatron to theoretical predictions. Event generators such as PYTHIA and Monte Carlo programs such as MCFM model the processes under consideration (i.e., the hard-scatter and prompt decays) [45], [46]. Additional software specific to each experiment is used to describe the interactions of the final state particles with the detector, and other effects such as electronics noise. The result of this process is a simulated raw data set that is processed by the same reconstruction program (see Chapter 3) used on collider data. Imperfect modeling of the detector causes the simulated data to have resolutions and efficiencies that are frequently superior to those observed in the laboratory. Therefore, corrections are applied to the reconstructed physics objects in the simulation.

\subsection{Simulation Software}

The PYTHIA program [45] is a general purpose event generator. It combines calculations of hard-scatter processes $\left(e . g ., q \bar{q}^{\prime} \rightarrow \rho_{T} \rightarrow W \pi_{T} \rightarrow \mu \nu b \bar{b}\right)$ at leading order in pertubation theory with a non-perturbative model of the evolution of final state partons into jets (i.e., the hadronization process). Since the Tevatron is a $p \bar{p}$ collider, it is also neccessary to account for the composite nature of the initial state particles. This is accomplished via an interface to parton distribution functions, which describe the probability to encounter a quark or gluon of a particular flavor and momentum inside a proton. In this dissertation, the CTEQ5L, leading order, distribution function is used, although others are available [47].

PYTHIA does not implement calculations of all the physics processes relevant to this dissertation. Furthermore, it is a leading order event genera- 
tor, and, thus, does not correctly model events with high jet multiplicities. Because such events usually involve the production of high energy gluons, a proper description relies on calculations at higer orders of perturbation theory. To address these issues, the COMPHEP [48] and ALPGEN [49] event generators are used. COMPHEP provides the capability of calculating the tree-level Feynman diagrams (i.e., diagrams with no internal loops) for arbitrary initial and final states. ALPGEN is a next-to-leading order, though still tree-level, event generator. Therefore, it improves on description from PYTHIA of events with multiple final state partons. Both COMPHEP and ALPGEN utilize the PYTHIA hadronization libraries and the CTEQ5L parton distribution functions.

The boundaries between the perturbative radiation of hard gluons modeled by ALPGEN and the softer partons emitted in the hadronization described by PYTHIA is somewhat ambiguous. Therefore, care must be taken to avoid double counting multi-jet events: a low jet multiplicity PYTHIA sample will contain a few events that have many jets and are thus also described by a high multiplicity ALPGEN sample. The MLM matching scheme is used to avoid this danger [50].

As an illustrative example, this analysis makes use of an inclusive $W$ sample generated with PYTHIA and a $W$ plus two parton $(W j j)$ sample from ALPGEN. The partons that are generated after the PYTHIA showering, but before the formation of hadrons, are clustered into jets with a $k_{T}$ algorithm, which is an alternative to the cone algorithm used in the event reconstruction). Events in the inclusive $W$ sample with at least two jets are discarded. Likewise, events in the ALPGEN sample with fewer than two jets are also discarded.

PYTHIA and COMPHEP, in addition to being event generators are also Monte Carlo programs that can be used to evaluate the total cross-section integrals. However, next to leading order programs, such as MCFM [46], provide more accurate results for implemented processes.

Once an event has been generated, it is neccessary to simulate the response of the DØ detector. This is accomplished by the DØGstar and DØSim programs. The GEANT libraries provide a description of high energy particle interactions with matter; these are used by DØGstar to determine the deposition of energy in the various detector components, including material in uninstrumented regions. DØSim simulates the digitization process, the electronics noise, and overlays minimum bias events. Such events may be obtained from collider data or separately simulated. For all of the simulated samples used herein, the numer of overlaid minimum bias interactions to overlay for a given event was chosen from a Poisson distribution with a mean of 0.8 . 


\subsection{Simulation Corrections}

Even if the event generator provides a good simulation of the underlying physics, the complete simulation may not accurately reproduce the data. In such cases, the problem may be traced to an imperfect model of the detector. Therefore, it is convenient to develop empirically parameterized corrections to the simulations that are applied at the analysis stage. Broadly, these corrections may be divided into two categories: the simulation may have different energy and momentum resolutions from the data; it may also have a different reconstruction efficiency (i.e., the probability to reconstruct a given physics object from the raw data). In both cases, the simulation normally has finer resolutions and higher efficiencies than collider data.

\subsubsection{Resolution Corrections}

The spatial resolution of the detector is generally modeled well and not in need of corrections. In this dissertation, the primitive kinematic variables used are the muon and jet momenta, and the missing transverse energy. Resolution corrections are applied by adding a random number from a gaussian distribution to the measurement; this process is called smearing. The width and mean of the distribution are obtained by comparing collider data and simulations for some control sample (the mean is normally zero). All changes are propagated to the relavent quantities (e.g., the mass variables computed from jet momenta, or the missing transverse energy from all other momentum measurements).

The muon momentum resolution may be studied in $Z \rightarrow \mu \mu$ events. Muon momenta are obtained from their associated tracks. Thus, muons inherit the track momentum resolution, which is gaussian in $q / p_{T}$, where $q$ is the track charge and $p_{T}$ is its transverse momentum. A smearing gaussian centered at zero with a width of $0.0022 \mathrm{c} / \mathrm{GeV}$ reproduces the width of the $\mathrm{Z}$ peak in collider data [51].

Since jet momenta are measured from the calorimeter, the $p_{T}$ resolution $\sigma_{p_{T}}$ may be parameterized as

$$
\sigma_{p_{T}}^{2}=N^{2}+S^{2} p_{T}+C^{2} p_{T}^{2}
$$

where $N, S$ and $C$ represent the contributions from electronics noise, statistical fluctuations in the jet shower evolution, and calibration errors, respectively [24]. The resolution is studied in events with a jet recoiling in $\phi$ against a photon (for low energies) or another jet (for $p_{T}>\sim 50 \mathrm{GeV} / c$ ) from simulated and collider data sets. These are the same samples used to generate the jet quality 


\begin{tabular}{|c|c|c|c|c|c|c|}
\hline & \multicolumn{3}{|c|}{ Simulated Data } & \multicolumn{3}{c|}{ Collider Data } \\
\hline$\left|\eta_{\text {det }}\right|$ range & $\mathrm{N}$ & $\mathrm{S}$ & $\mathrm{C}$ & $\mathrm{N}$ & $\mathrm{S}$ & $\mathrm{C}$ \\
\hline $0.0-0.5$ & 4.26 & 0.658 & 0.0436 & 5.05 & 0.753 & 0.0893 \\
$0.5-1.0$ & 4.61 & 0.621 & 0.0578 & 0 & 1.20 & 0.0870 \\
$1.0-1.5$ & 3.08 & 0.816 & 0.0729 & 2.24 & 0.924 & 0.135 \\
$1.5-2.0$ & 4.83 & 0 & 0.0735 & 6.42 & 0 & 0.0974 \\
\hline
\end{tabular}

Table 4.1: Parameterization of jet momentum resolutions for collider and simulated data [35].

variable distributions discussed in Section 3.3. In the absence of neutrinos, the true transverse momenta of the jet-jet or jet-photon system should be zero; thus, the energy resolution may be measured from the width of the observed momentum asymmetery distribution in such events [35]. The parameters are measured separately for collider and simulated data, and in several bins of detector $\eta$, as shown in Table 4.1. For a given simulated jet, the collider data and simulated resolutions $\sigma_{p_{T}}^{\text {data }}$ and $\sigma_{p_{T}}^{\text {sim }}$ are computed. The transverse momentum is then smeared with a gaussian of width $\sigma=\sqrt{\sigma_{p_{T}}^{\text {data }^{2}}-\sigma_{p_{T}}^{\text {sim }}}$.

The reproduction of the missing transverse energy was also studied in a $Z \rightarrow \mu \mu$ sample. Because such events have no neutrinos, any observed $\mathbb{F}_{T}$ is primarily due to energy mismeasurements. The missing $E_{T}$ components $\mathbb{E}_{u}$ and $E_{v}$ that are, respectively, parallel and perpendicular to the transverse momentum of the $Z\left(p_{T}^{Z}\right)$, are determined. The muons are normally back to back in $\phi$, so $p_{T}^{Z}$ is expected to be perpendicular to the muon momenta. Therefore, $\mathbb{E}_{u}$ is dominated by the calorimeter resolution, the energy from the underlying event, and any minimum bias collisions. However, mismeasurements of the muon momenta are the primary contribution to $\not_{v}$. Shown in Figure 4.1 are the $\mathbb{E}_{u}$ and $\mathbb{E}_{v}$ distributions for collider and simulated data. The distributions are in good agreement, and no smearing is required for the missing transverse energy measurement.

\subsubsection{Efficiency Scale Factors}

In addition to smearing the measured energies and momenta of reconstructed physics objects, it is also neccessary to compensate for reconstruction and identification efficiencies that are larger in the simulation than in the recorded data. The ratio of the two is a scale-factor that is a function of momentum and location. In this dissertation, the scale-factors are used in a 

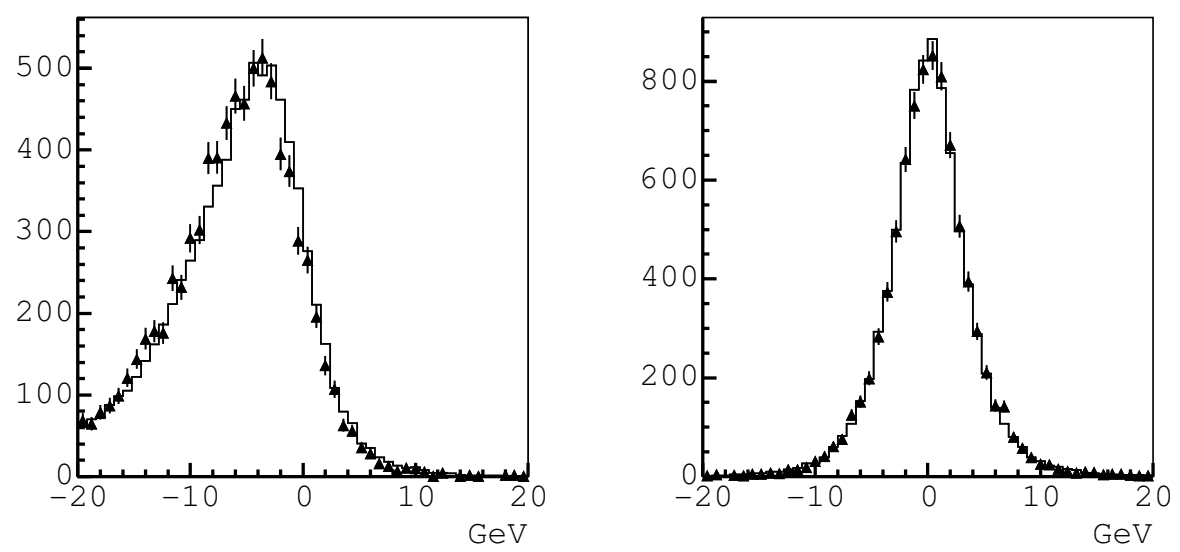

Figure 4.1: Distributions of $E_{u}$ (left), the component of the missing $E_{T}$ parallel to the $Z$ transverse momentum and $E_{v}$ (right), the perpendicular component. Recorded collider data, obtained from $Z \rightarrow \mu \mu$ events, is shown with points, while the solid histograms represent simulated events, normalized by area to the data.

random removal algorithm: for each jet,a random number between zero and one is generated. If that random number is greater than the scale-factor for that jet, then the jet is removed from the event. The scale-factors for primary vertices and jets are discussed in this section. No scale-factor is neccessary for muons because of the normalization procedure described in Section 6.2.1.

In events with no jets, a scale-factor of 0.954 was used for primary vertex reconstruction. This number was obtained by comparing the fractions of $W \rightarrow$ $\mu \nu$ plus 0 jet events with no primary vertices in recorded and simulated data. In events with jets no correction is required [51], [52].

The scale-factor for jet reconstruction was determined by running a jet finding algorithm on the reconstructed tracks [53]. The efficiency for reconstructing jets in the calorimeter was then measured in events with a photon recoiling against a track jet (see Section 5.1. The scale-factor $(S F)$ was parameterized as an error function:

$$
S F=\frac{p_{2}}{2} \operatorname{erf}\left(\frac{p_{T}-p_{0}}{p_{1} \sqrt{p_{T}}}\right)
$$

where $p_{i=0,1,2}$ are parameters measured in three $\eta$ regions that roughly correspond to the central and endcap calorimeters as well as the inter-cryostat region. The parameter values are reported in Table 4.2. 


\begin{tabular}{|c|c|c|c|}
\hline$|\eta|$ region & $p_{0}$ & $p_{1}$ & $p_{2}$ \\
\hline$<0.8$ & $9.8 \pm 2.7$ & $3.38 \pm 0.73$ & $1.0105 \pm 0.0015$ \\
$0.8-1.5$ & $15.0 \pm 2.3$ & $1.96 \pm 0.74$ & $0.9271 \pm 0.0024$ \\
$>1.5$ & $13.5 \pm 3.3$ & $2.2 \pm 1.0$ & $0.9828 \pm 0.0022$ \\
\hline
\end{tabular}

Table 4.2: Parameters used to determine the jet reconstruction scale-factor. Only uncertainties from the fitting process are shown [53]. 


\section{Chapter 5}

\section{Heavy Quark Identification}

The technipions searched for in this dissertation decay primarily via the channels $\pi_{T}^{0} \rightarrow b \bar{b}$ and $\pi_{T}^{ \pm} \rightarrow b \bar{c}$, as implied by equation 1.28. Identification of jets containing the heavy $b$ - or $c$-quarks is a useful tool in suppressing the large backgrounds from sources without any heavy flavor component. One example of such a source is continuum $\mathrm{W}(\rightarrow \mu \nu)$ plus light flavor jet production, which is the dominant background for this analysis.

Hadrons containing heavy quarks have lifetimes of roughly one picosecond; those with a $b$-quark live somewhat longer than those with a $c$-quark, due to a suppression factor from the quark mixing matrix. At the energies typical of the Tevatron, these hadrons will travel hundreds of micrometers before decaying. Jets with such particles may be identified through the reconstruction of a decay vertex that is sufficiently far from the primary interaction. Alternatively, the presence of a large number of tracks inconsistent with the primary interaction may be used to infer the presence of heavy quarks in a jet.

The latter approach is the one used by the Jet Lifetime Impact Parameter (JLIP) algorithm for the search presented here [54]. It constructs a probability, $\mathcal{P}_{\text {jet }}$ (also the called jet lifetime probability), that all of the tracks in a jet are consistent with the primary vertex. The jet is considered to be $b$-tagged if that probability is less than $0.3 \%$, although operating points with tighter or looser requirements may be chosen, based on the demands of the particular analysis. The algorithm is more efficient for identifying jets with $b-(\sim 55 \%)$ than $c$-quarks $(\sim 15 \%)$, due to the difference in their lifetimes.

The detailed modeling of tracks in jets is a difficult task, giving rise to large systematic uncertainties. To reduce these systematics and more accurately reproduce the actual $b$-tagging efficiencies, the JLIP tagger does not process simulated data considered for the Technicolor search. Instead, a tag rate function (TRF) is used to determine the probability that a jet of a given flavor will satisfy the tagging requirement. The jet flavor is determined by searching 
the record of generated partons for $b$ - or $c$-quarks within the jet cone. The tagging probability is used to calculate a weight for the contribution of each event to the total expected yield. The evaluation of the TRFs is discussed in Section 5.4.

\subsection{Track Jets and Taggability}

All heavy flavor identification algorithms rely on the use of tracking information; therefore, the presence of tracks associated with a jet is a prerequisite for tagging it. ${ }^{1}$ This requirement is enforced through the use of track jets. Track jets are reconstructed from tracks with at least one SMT hit and $p_{T}>0.5 \mathrm{GeV} / c$. Tracks are also required to pass within $0.2 \mathrm{~cm}$ of the primary vertex in the $x-y$ plane and $0.4 \mathrm{~cm}$ along the $z$ axis. A cone algorithm similar to the one described in Section 3.3, with $R_{\text {cone }}=0.5$, is used to cluster such tracks into jets [54]. At least two tracks are required, with one having a transverse momentum greater than $1 \mathrm{GeV} / c$. A calorimeter jet that is separated from a track jet by a distance of less than 0.5 in the $\eta-\phi$ plane is considered taggable.

It is necessary to correct for the difference in the efficiency of the taggability requirement between collider and simulated data. A scale-factor equal to $0.949-0.020 \eta^{2}$ is derived from a sample of jets recoiling against a $Z$ decaying to two muons [51]. This correction is applied by use of the random removal procedure described in Section 4.2.2.

\section{$5.2 V^{0}$ Rejection}

A potentially significant background to $b$ - and $c$-jet identification arises from light hadron decays and $\gamma \rightarrow e^{+} e^{-}$conversions, collectively referred to as $V^{0}$ particles. To understand this issue, a jet-triggered sample of high energy, taggable jets with $|\eta|<1.2$ was considered [54]. Invariant mass distributions of $p \pi$ and $\pi^{+} \pi^{-}$pairs, reconstructed from tracks with $p_{T}>1 \mathrm{GeV} / c$ and passing within $0.15 \mathrm{~cm}$ of the primary vertex (in the $x-y$ plane), are shown in Figure 5.1. Approximately $6-8 \%$ of such jets have a $V^{0}$ candidate, but this number rises to $20-25 \%$ for jets with a lifetime tag: $\mathcal{P}_{\text {jet }}<0.1 \%$. Tracks belonging to such candidates are removed from further consideration, thus reducing the

\footnotetext{
${ }^{1}$ In passing, it is noted that such a criterion also supresses the effects of calorimeter noise.
} 

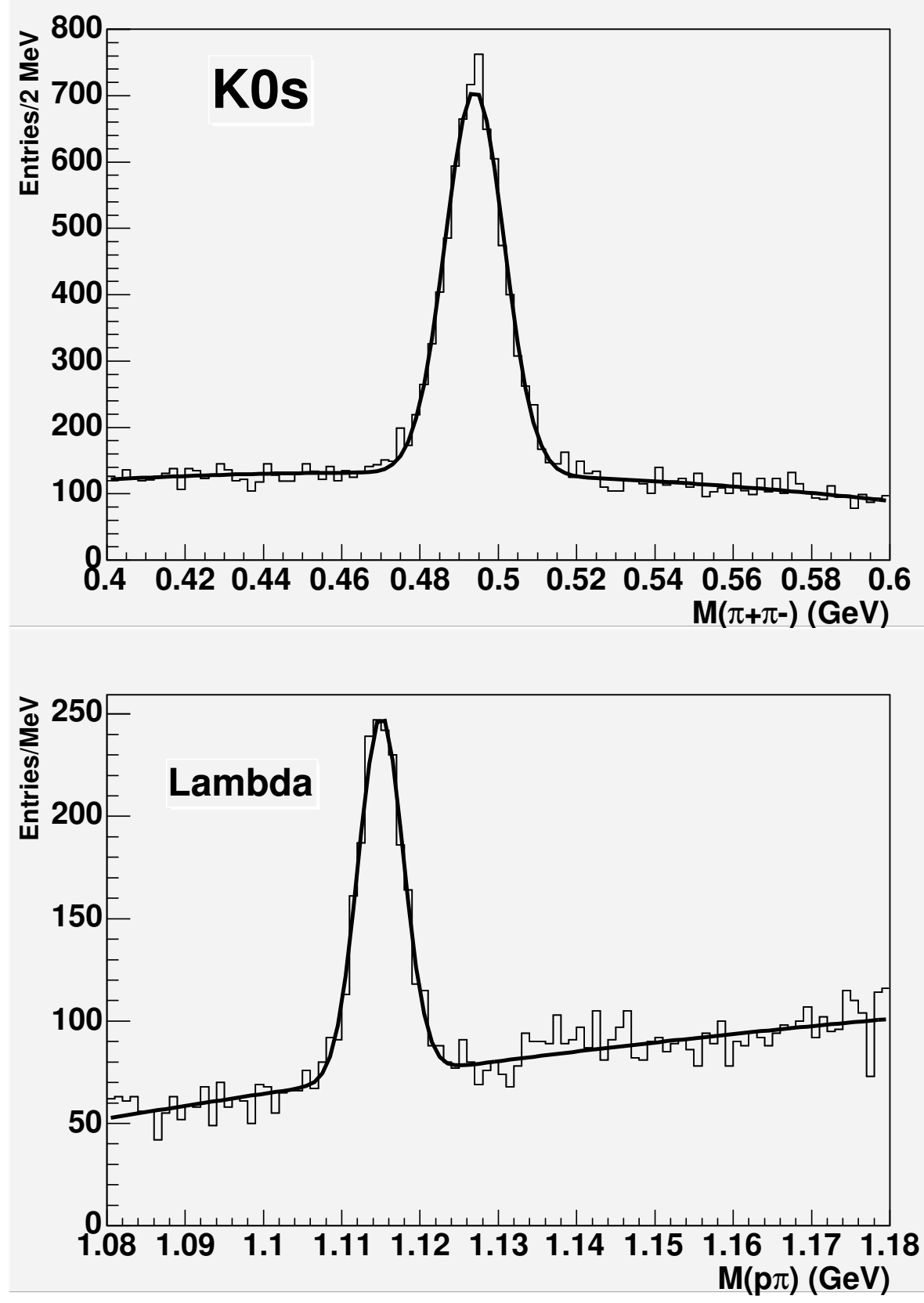

Figure 5.1: Invariant mass distributions of $\pi^{+} \pi^{-}$(top) and $\mathrm{p} \pi^{-}$(bottom) systems reconstructed from jet-triggered data [54]. The distributions are fit to a sum of a gaussian and polynomial function. 
tagging rate for jets without a heavy quark by $10 \%$ while leaving the efficiency for $b$-jets unchanged.

\subsection{The JLIP Tagging Algorithm}

The jet lifetime probability is constructed from the impact parameter (IP) of individual tracks: the distance to the point of closest approach between the track and the primary vertex. If this point is on the same side of the vertex as the jet, then the IP is taken to be positive. Otherwise, it is defined to be negative.

Tracks from the decay of a $b$ - or $c$-flavored hadron will tend to have a large positive IP. The principal background is from tracks where the IP has been mismeasured. Since the distribution for tracks from the primary vertex is approximately symmetric about zero, this background may be quantified by considering tracks with a negative impact parameter.

The impact parameter significance, $\mathcal{S}_{\mathcal{I P}}$, may be defined by dividing the IP by its uncertainty, $\sigma_{I P}$ :

$$
\mathcal{S}_{\mathcal{I P}} \equiv \frac{I P}{\sigma_{I P}}
$$

The probability distribution of $\mathcal{S}_{\mathcal{I P}}$ (shown in Figure 5.2 for tracks of various qualities), $\mathcal{R}_{\mathcal{I P}}\left(\mathcal{S}_{\mathcal{I P}}\right)$, may be integrated to determine the probability, $\mathcal{P}_{\text {trk }}$, that a track is consistent with the primary vertex:

$$
\mathcal{P}_{\text {trk }}\left(\mathcal{S}_{\mathcal{I P}}\right) \equiv \frac{\int_{-50}^{-\left|\mathcal{S}_{\mathcal{I P}}\right|} \mathcal{R}_{\mathcal{I P}}(s) d s}{\int_{-50}^{0} \mathcal{R}_{\mathcal{I P}}(s) d s}
$$

Distributions of $\mathcal{P}_{t r k}$ from jet-triggered and simulated data are shown in Figure 5.3. Note that tracks from heavy quark jets have a peak near zero. The peak is present, but much smaller for light quark jets. A quantity, $\mathcal{P}_{\text {jet }}^{+}$, calculated from all tracks with positive IPs that gives the probability that they originated from the primary vertex:

$$
\mathcal{P}_{j e t}^{+} \equiv \Pi^{+} \times \sum_{j=0}^{N_{t r k}^{+}-1} \frac{\left(-\log \Pi^{+}\right)^{j}}{j !}
$$

with

$$
\Pi^{+} \equiv \prod_{i=1}^{N_{t r k}^{+}} \mathcal{P}_{t r k}
$$



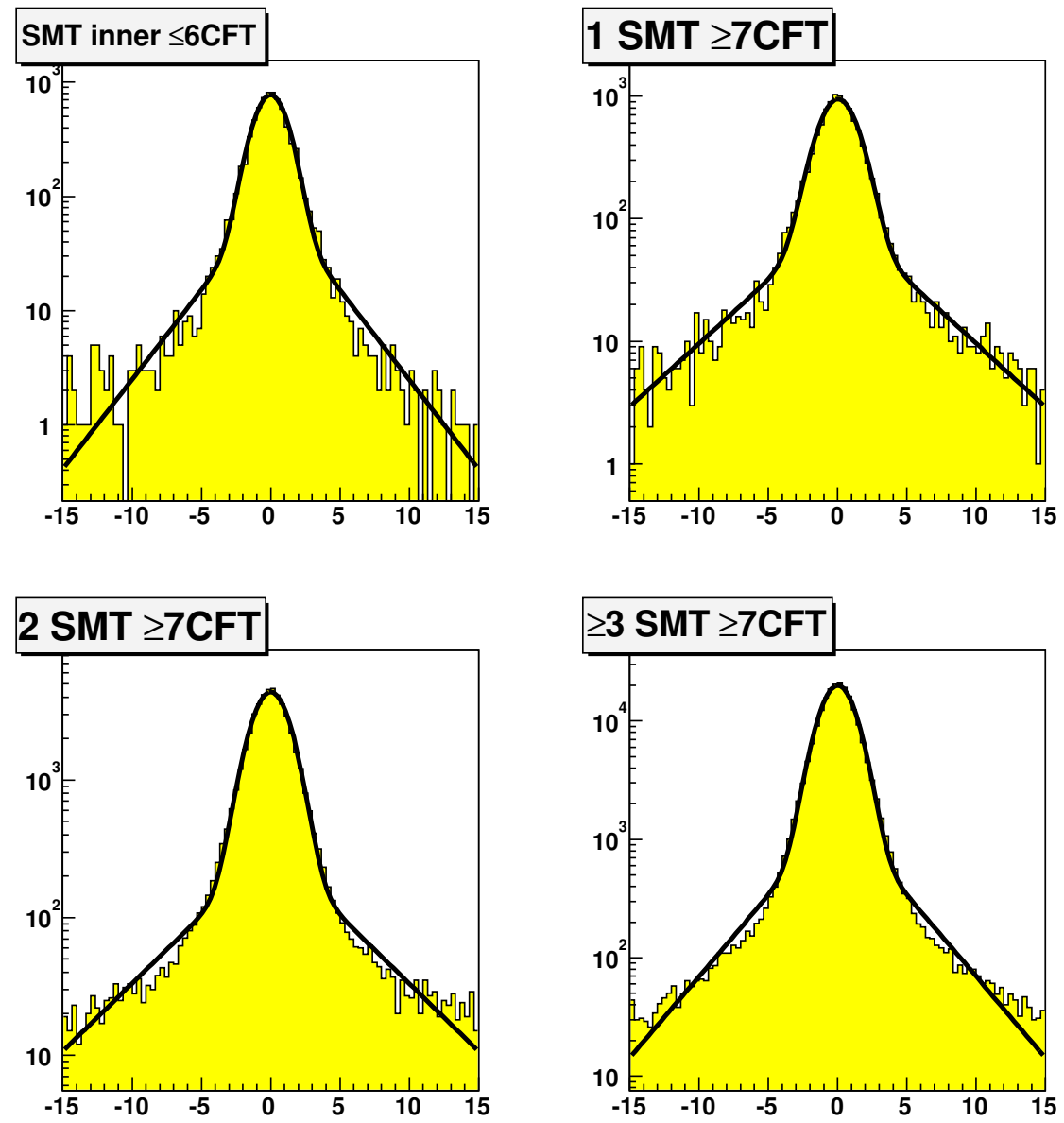

Figure 5.2: Distributions of the impact parameter significance for tracks with different numbers of SMT and CFT hits. Distributions are fit to the sum of a gaussian (to describe the peak) and an exponetial (to model the tails). The fits are all good out to $\pm 5 \sigma$, encompassing the vast majority of the tracks [55]. 
where $N_{t r k}^{+}$is the number of positive IP tracks.

A similar quantity, $\mathcal{P}_{\text {jet }}^{-}$, is determined from tracks with a negative IP. Distributions of these variables are plotted in Figure 5.4. A jet is considered $b$-tagged if it satisfies $\mathcal{P}_{\text {jet }} \equiv \mathcal{P}_{\text {jet }}^{+}<0.3 \%$.

\subsection{The JLIP Tag Rate Functions}

The performance of the JLIP algorithm may be stated in terms of the efficiencies to tag $b$-, $c$ - or light flavor jets (the TRFs). These efficiencies may be functions of the candidate jet momentum and pseudorapidity. The mistag rate for falsely tagging jets without a heavy quark is measured in data with inputs from the simulation to correct for the true flavor composition of a jet. The $b$ - and $c$-tagging rates were measured in simulated events. A correction $\left(S F_{b}\right)$ was derived by comparing the $b$-tagging efficiency to collider data [55]. All TRFs and corrections are assumed to be functions of jet transverse energy and pseudorapdity.

\subsubsection{Evaluation of the Mistag Rate}

The light flavor mistag rate is evaluated with negatively tagged jets: those jets satisfying $\mathcal{P}_{\text {jet }}^{-}<0.3 \%$ (tagging with $\mathcal{P}_{\text {jet }}^{+}$is also called positive tagging). The probability, $\epsilon_{\text {data }}^{-}$, in events recorded with a jet trigger, to negatively tag a jet provides an approximation of the mistag rate, $\epsilon_{\text {light }}$. Two multiplicative corrections, $F_{h f}$ and $F_{l l}$, are derived from a simulated multi-jet sample [55].

The jet-triggered data will have some heavy quark content that could bias the mistag rate. The first correction factor, $F_{h f}$, is the ratio of the negative tag rates for light flavor jets to the rate for all jets, as measured in the simulated sample.

The second correction factor is necessary because of the presence of longlived hadrons in jets. These hadrons cause the positive and negative tagging rates to differ. The ratio, $F_{l l}$, of the two efficiencies is applied to compensate for this effect.

Both $F_{h f}$ and $F_{l l}$ are determined as functions of jet $E_{T}$ and $\eta$. The fully corrected mistag rate, $\epsilon_{\text {light }}=\epsilon_{\text {data }}^{-} \times F_{h f} \times F_{l l}$, is shown in Figure 5.5. Note that rates are shown for several operating points, including to the $\mathcal{P}_{\text {jet }}<0.3 \%$ criterion used in this dissertation.

The energy and pseudorapidity dependence of the mistag rate was assumed to factorize to the following function: 


\section{track significance probability}
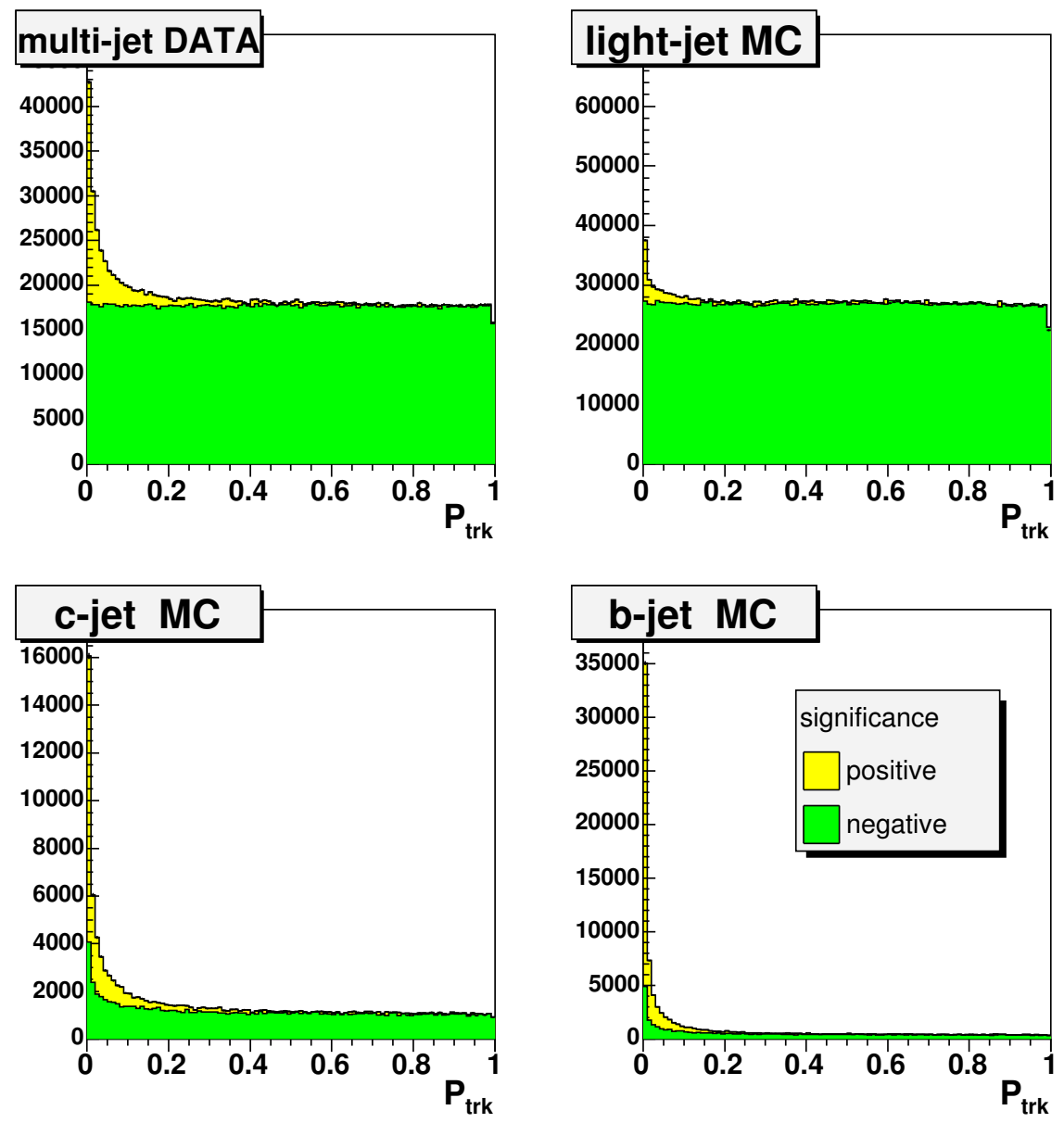

Figure 5.3: Distributions of $\mathcal{P}_{\text {trk }}$, for positive (yellow, lighter) and negative (green, darker) impact parameter tracks. The top left displays the distribution for jet-triggered data. Simulated events for light flavor jets (top right), as well as jets with $c$ - (bottom left) and $b$-quarks (bottom right) are also shown [54]. 

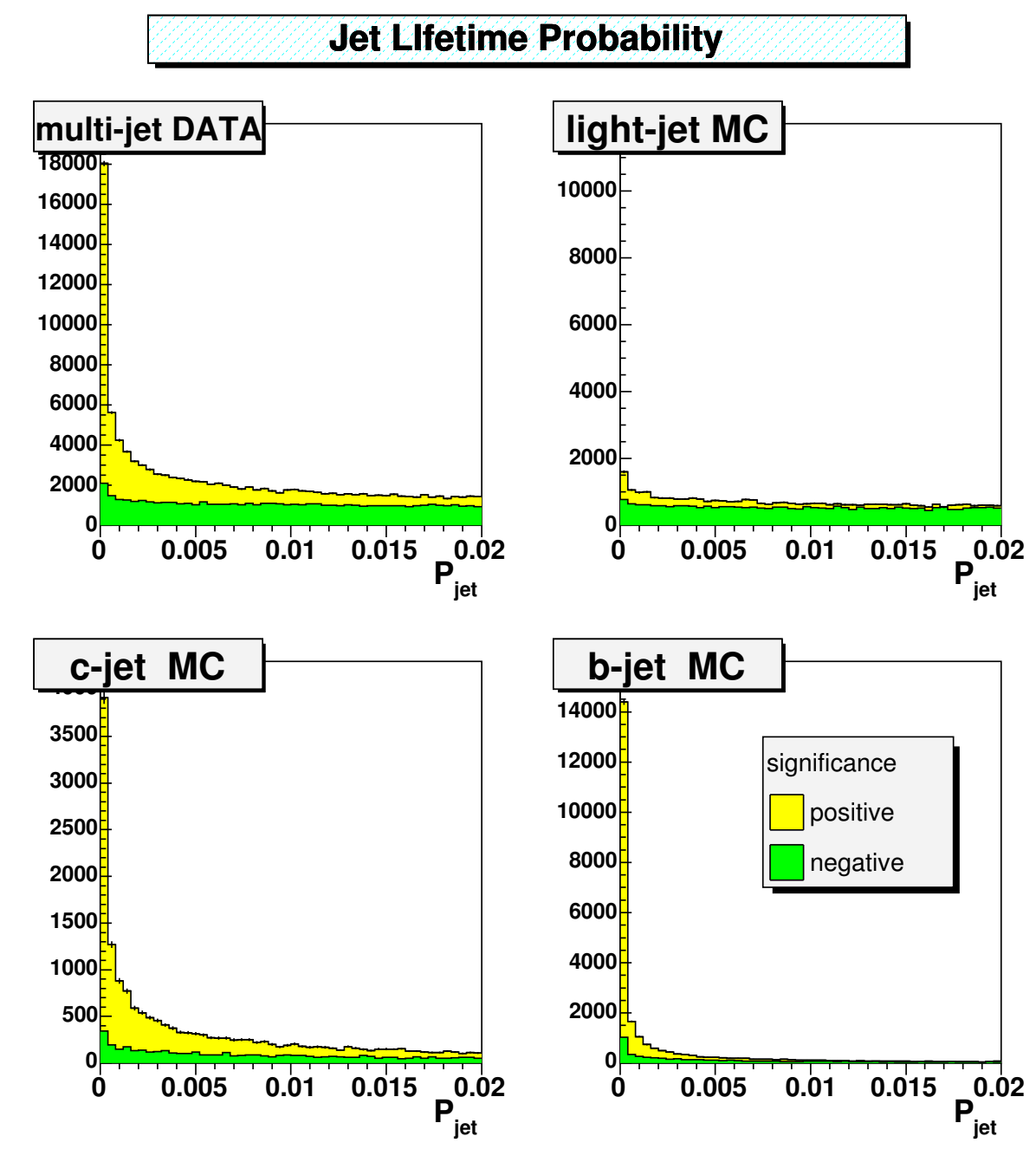

Figure 5.4: Distributions of $\mathcal{P}_{\text {jet }}^{+}$(yellow, lighter) and $\mathcal{P}_{\text {jet }}^{-}$(green, darker), for data recorded with a jet trigger (top left), simulated light flavor jets (top right), $c$-jets (bottom left), and $b$-jets (bottom right) [54]. 

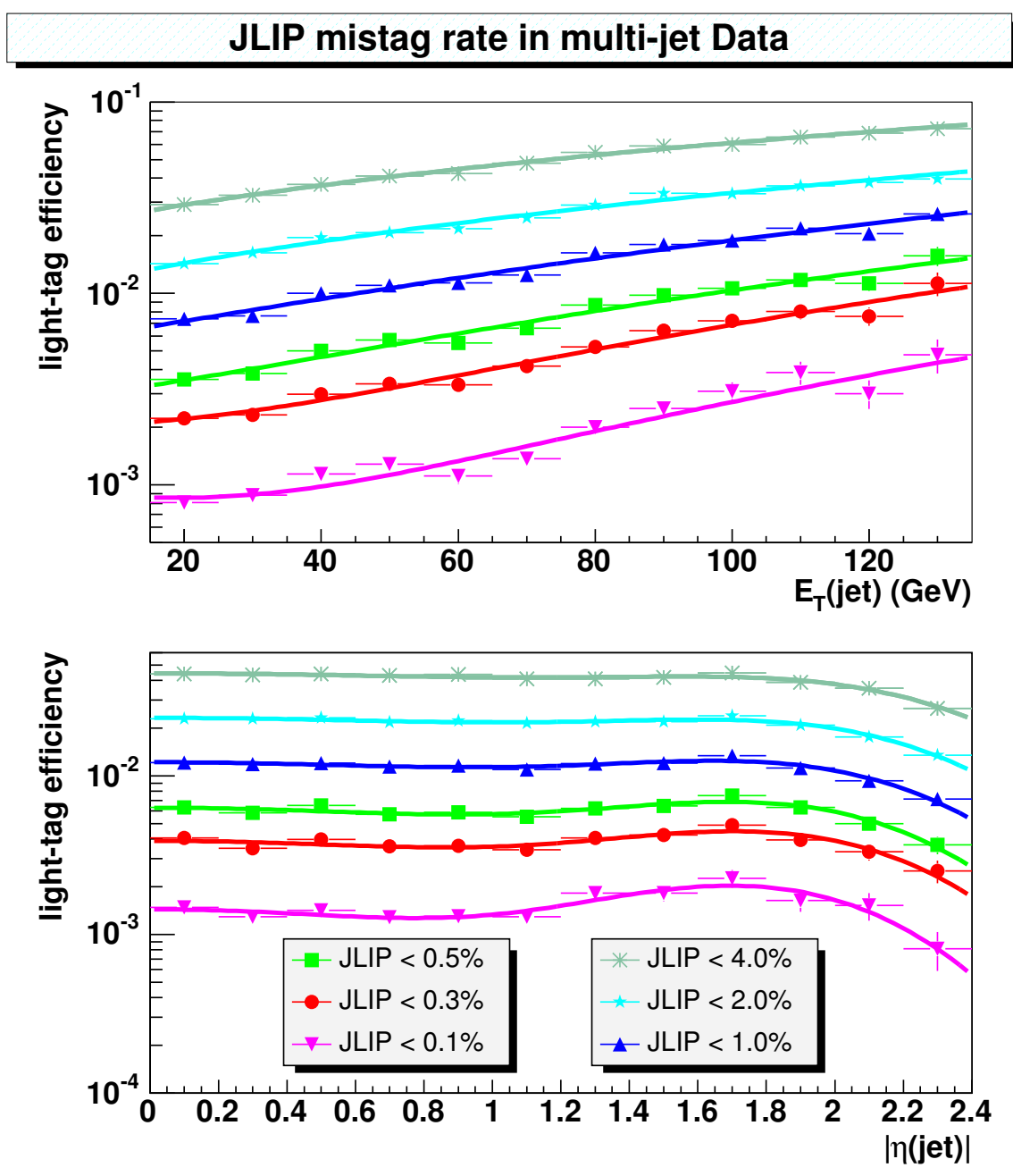

Figure 5.5: Light flavor tag rates, as a function of jet transverse energy (top) and pseudorapidity (bottom) [55]. 


\begin{tabular}{|l|l|}
\hline Parameter & Value \\
\hline$p_{0}$ & $5.54_{-0.61}^{+0.65} \times 10^{-4}$ \\
$p_{1}$ & $-0.998 \pm 0.042$ \\
$p_{2}$ & $4.00_{-0.14}^{+0.12}$ \\
$p_{3}$ & $11.60_{-0.68}^{+0.63}$ \\
$p_{4}\left(\mathrm{GeV}^{-1}\right)$ & $-1.3_{-2.3}^{+2.9} \times 10^{-3}$ \\
$p_{5}\left(\mathrm{GeV}^{-2}\right)$ & $2.44_{-0.22}^{+0.18} \times 10^{-4}$ \\
\hline
\end{tabular}

Table 5.1: Fit parameter values, with full systemic uncertainties, for the light flavor tag rate function [56].

$$
\epsilon_{\text {light }}=p_{0} \exp \left(-\frac{\eta^{4}}{p_{3}}\right)\left(\eta^{4}+p_{1} \eta^{2}+p_{2}\right)\left(1+p_{4} E_{T}+p_{5} E_{T}^{2}\right)
$$

The parameter values were determined by a fit to the data (the functional form is purely empirical) and are listed in Table 5.1 [56]. As a cross-check, the analysis was repeated on jets from events satisfying an electromagnetic trigger. The final tag rate function was taken to be the average of the two fits, with the difference included as a systematic uncertainty. Uncertainties were also calculated for the flavor composition of the simulated multi-jet sample and the factorization assumption. The full, average, systematic uncertainty for the mistag rate is $9.3 \%$ [55].

\subsubsection{Determination of the Tagging Efficiencies}

The efficiencies to tag $b$ - and $c$-jets, as measured from simulated data, are plotted in Figure 5.6. To compensate for differences between the simulation the actual collider data, a scale factor, $S F_{b}$, is derived for the $b$-tagging efficiency. This scale factor is applied to both the $b$ - and $c$-tagging efficiencies to determine the appropriate TRFs [55].

The determination of $S F_{b}$ requires evaluating of the $b$-tagging efficiency from collider data. This is accomplished by considering the effects of two different tagging algorithms on two samples with differing signal (in this case $b$-jet) and background (charm and light jet) compositions [57]. The application of all tagger combinations yields a system of eight linear equations with eight unknowns, which include the tagging efficiencies. Extraction of the efficiencies is then a matter of solving the equations. 

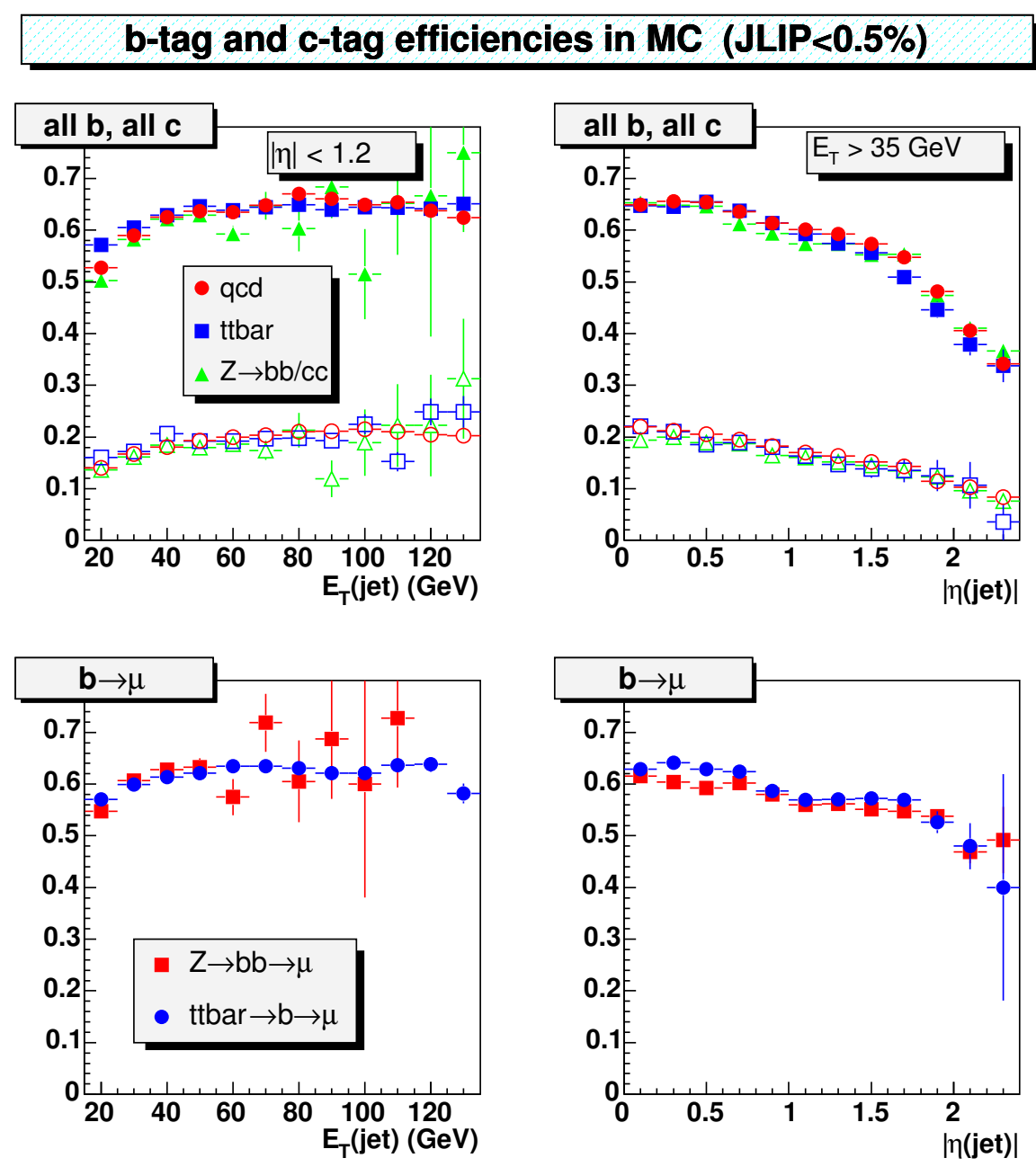

Figure 5.6: Tagging efficiencies for $b$ - (solid points) and $c$-jets (open points) measured in simulated data for various processes. The top plots show the results for all heavy flavor jets, while the bottom plots are restricted to $b$-jets with a muon in the decay chain [55]. 
The first sample consists of jets with an associated loose muon $\left(p_{T}^{\mu}>4 \mathrm{GeV} / c\right.$, $\Delta R(\mu$, jet $)<0.5)$ enriched in heavy flavor content from semileptonic $B$ decays. A subset of these jets, forming the second sample, recoil against an additional jet that has been tagged with $\mathcal{P}_{\text {jet }}<1 \%$. The first tagger requires that the component of the muon momentum, $p_{T}^{r e l}$, transverse to the associated jet axis, be greater than $0.7 \mathrm{GeV} / c$. Since the $p_{T}^{r e l}$ distribution for light flavor and $c$-jets is the same [57], they also have the same tagging efficiencies. To extract the desired $b$-tagging rate, the second tagger must be the JLIP algorithm, with a probability requirement of $0.3 \%$.

The number of events in the first and second samples are given by $n$ and $p$, respectively. Superscripts are used to indicate the tagging algorithm (or combination) applied. Thus, $n^{J L I P}$ is the total number of jets in the first (muon-in-jet) sample, while $p^{J L I P, \mu}$ gives the number of jets in the second (opposite-tag) sample tagged by both the JLIP and $p_{T}^{r e l}$ methods. Subscripts are used to indicate flavor composition, and $\epsilon_{Y}^{X}$ gives the proability to tag a jet. For example, $n_{b}$ represents the number of $b$-jets in the first sample, and $\epsilon_{c l}^{J L I P}$ indicates the (flavor-averaged) efficiency for tagging light or charm jets. The eight unknowns are the four flavor contributions $\left(n_{b}, n_{c l}, p_{b}, p_{c l}\right)$ and the four efficiencies $\left(\epsilon_{b}^{J L I P}, \epsilon_{c l}^{J L I P}, \epsilon_{b}^{\mu}, \epsilon_{c l}^{\mu}\right)$.

With these definitions, the equations to be solved are:

$$
\begin{aligned}
n & =n_{b}+n_{c l} \\
p & =p_{b}+p_{c l} \\
n^{\mu} & =\epsilon_{b}^{\mu} n_{b}+\epsilon_{c l}^{\mu} n_{c l} \\
p^{\mu} & =\epsilon_{b}^{\mu} p_{b}+\epsilon_{c l}^{\mu} p_{c l} \\
n^{J L I P} & =\epsilon_{b}^{J L I P} n_{b}+\epsilon_{c l}^{J L I P} n_{c l} \\
p^{J L I P} & =\beta \epsilon_{b}^{J L I P} p_{b}+\alpha \epsilon_{c l}^{J L I P} p_{c l} \\
n^{\mu, J L I P} & =\kappa_{b} \epsilon_{b}^{\mu} \epsilon_{b}^{J L I P} n_{b}+\kappa_{c l} \epsilon_{c l}^{\mu} \epsilon_{c l}^{J L I P} n_{c l} \\
p^{\mu, J L I P} & =\kappa_{b} \beta \epsilon_{b}^{\mu} \epsilon_{b}^{J L I P} p_{b}+\kappa_{c l} \alpha \epsilon_{c l}^{\mu} \epsilon_{c l}^{J L I P} p_{c l}
\end{aligned}
$$

where $\alpha, \beta, \kappa_{b}$ and $\kappa_{c l}$ are parameters that describe the validity of certain assumptions. When the assumptions are true, the parameters are equal to one:

- The charm to light flavor ratio is the same in both samples. If this is not the case it is corrected for by $\alpha$. No correction is needed when only the the $p_{T}^{r e l}$ method is applied, because it tags charm and light jets with equal probability. 
- The JLIP tagging efficiency is the same in both samples. The accuracy of this assumption is measured by $\beta$.

- The tagging rates for the two algorithms are assumed to be uncorrelated. Any correlation is described by $\kappa_{b}$ and $\kappa_{c l}$ for the appropriate jet flavors.

These assumptions are validated in the simulation. The parameter $\alpha$ is set to unity and varied between 0.2 and 1.8. This yields a (relative) systematic of $0.3 \%$ on the tagging efficiencies. The $\beta$ factor is observed to have a constant value of $1.016 \pm 0.003$ (stat). The correlation $\kappa_{b}$ has a slight linear dependence on jet energy, ranging from 1.01 for $E_{T}=20 \mathrm{GeV}$ to 0.94 at $E_{T}=80 \mathrm{GeV}$. The statistical uncertainly on the slope is used to assign a systematic of \pm 0.004 to the tag rate. The light and charm jet correlation factor was found to be flat: $k_{c l}=0.90 \pm 0.05$.

The scale factor, $S F_{b}$, is obtained by dividing the $b$-tagging efficiency measured from collider data by the efficiency measured in the simulation. The TRFs for $b$ - and $c$-jets, shown in Figures 5.7 and 5.8, are obtained by multipying the simulated rate by the scale factor; in the case of $b$-jets, this is identical to using the rate measured from data. The rates for both flavors are separately fit to the function:

$$
\epsilon_{\text {heavy }}=\exp \left(-\frac{\eta^{4}}{p_{3}}\right) p_{0}\left(\eta^{4}+p_{1} \eta^{2}+p_{2}\right)\left(1-p_{4} \exp \left(-\frac{1}{2}\left(\frac{E_{T}-p_{5}}{p_{6}}\right)^{2}\right)\right)
$$

where the results from the fit are shown in Table 5.2. Systematic uncertainties include: variations in the $\alpha, \beta, \kappa_{b}$ and $\kappa_{c l}$ parameters; the $E_{T}$ and $\eta$ factorization hypothesis for the TRFs; and the variation of the measured tagging efficiencies among the simulated samples. The $p_{T}^{r e l}$ requirement was also varied from $0.5 \mathrm{GeV} / c$ to $0.9 \mathrm{GeV} / c$. The average uncertainties for the $b$ - and $c$-jet TRFs are $2.9 \%$ and $3.4 \%$, respectively. For $S F_{b}$, the value is $1.7 \%$. 

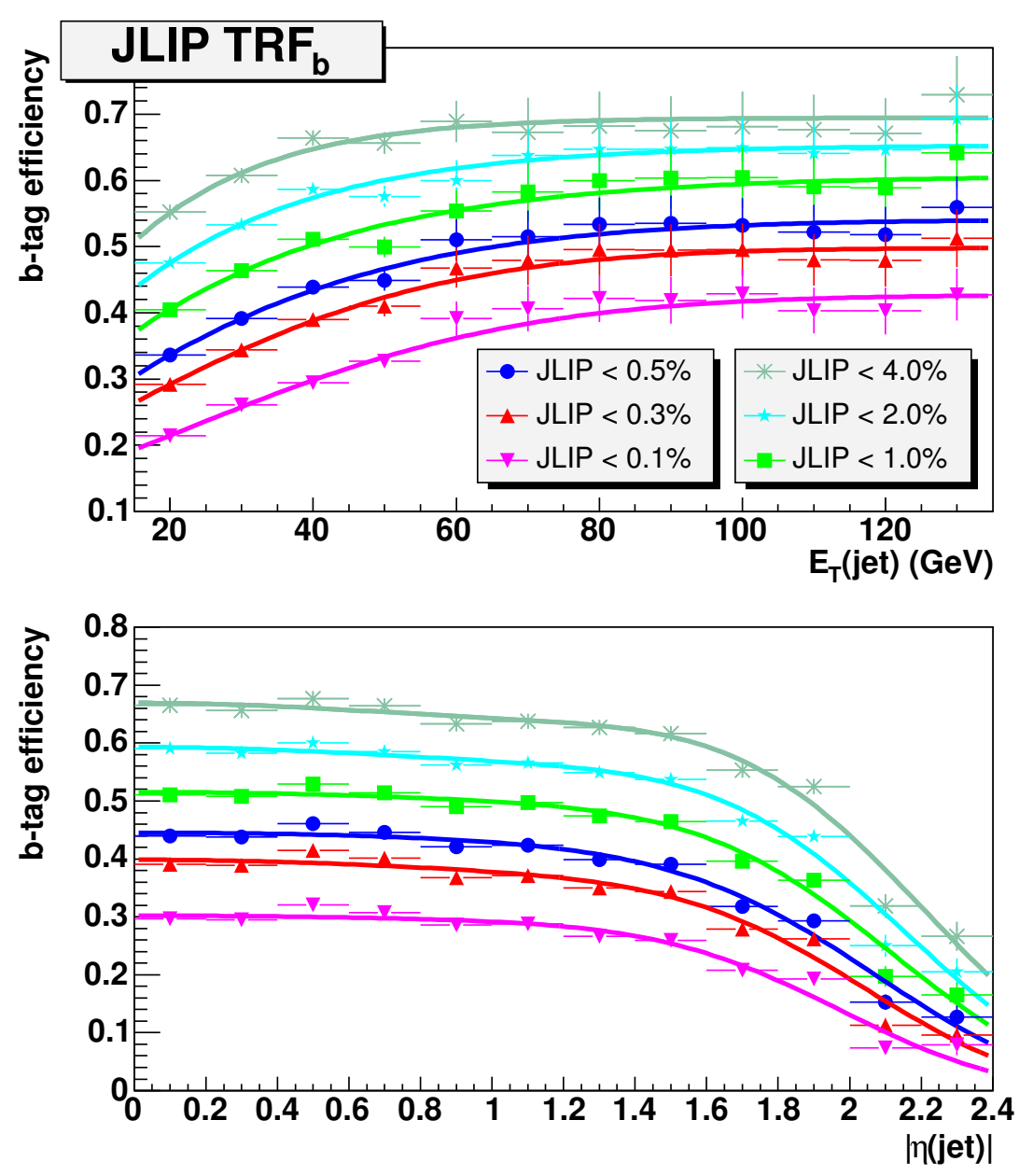

Figure 5.7: Final $b$-jet tag rate functions for various JLIP requirements [55]. 

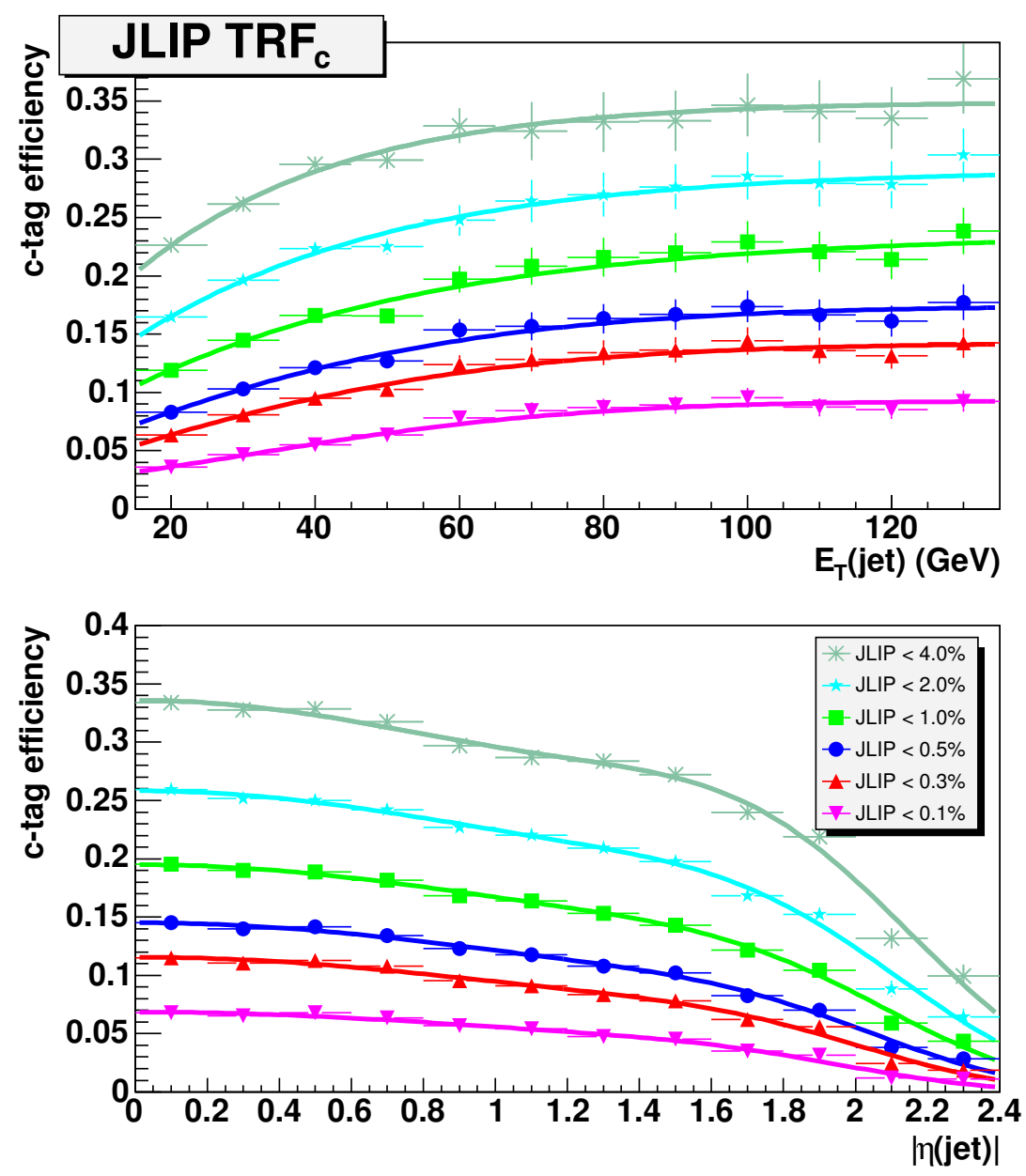

Figure 5.8: Corrected $c$-jet TRFs for different JLIP operating points [55]. 


\begin{tabular}{|l|ll|}
\hline & \multicolumn{2}{|c|}{ Value } \\
\hline Parameter & $b$-jets & $c$-jets \\
\hline$p_{0}$ & $5.80_{-0.38}^{+0.25} \times 10^{-2}$ & $2.336_{-0.039}^{+0.018} \times 10^{-2}$ \\
$p_{1}$ & $-0.578_{-0.058}^{+0.075}$ & $-1.617_{-0.070}^{+0.061}$ \\
$p_{2}$ & $9.6_{-1.4}^{+1.9}$ & $7.59_{-0.80}^{+0.93}$ \\
$p_{3}$ & $10.00_{-0.83}^{+0.98}$ & $8.67_{-0.64}^{+0.71}$ \\
$p_{4}$ & $0.835_{-0.015}^{+0.060}$ & $1.46_{0.21}^{+-0.11}$ \\
$p_{5}(\mathrm{GeV})$ & -33.333412 & -65.098503 \\
$p_{6}(\mathrm{GeV})$ & $45.1_{-5.3}^{+4.5}$ & $61.2_{-5.9}^{+5.2}$ \\
\hline
\end{tabular}

Table 5.2: Fit parameter values, with full systematic uncertainties for the the $b$ - and $c$-jet tag rate functions. The uncertainties on $p_{5}$ are approximately $\pm 1 \times 10^{-6}$ for both flavors [56]. 


\section{Chapter 6}

\section{Event Selection}

The final states for the Technicolor channels considered contain a muon and a muon-neutrino from the decay of a $W$, as well as two jets from technipion decays (each containing a $b$ - or $c$-quark, depending on the $\pi_{T}$ charge state). Events consistent with this final state are identified. To further enhance the sensitivity to the signal, optimized criteria on topological variables (most notably the invariant mass of the dijet system as well as that of the $\mu \nu b j$ system) are used to supress background contributions. Before discussing these selection requirements, it is neccessary to describe the recorded and simulated data sets analyzed.

\subsection{Data Samples}

The collider data set analyzed consists of an integrated luminosity of 291 $\mathrm{pb}^{-1}$, recorded between April 2002 and August 2004 with one of the following single muon triggers:

MU_W_L2M5_TRK10: A muon track must be found by the Level 1 trigger using only the scintillation counters within the CFT acceptance $\left(\left|\eta_{\text {det }}\right|<1.5\right)$. At Level 2 , a medium quality muon with a $p_{T}>5 \mathrm{GeV} / c$ (as measured by the toroid) must be reconstructed. Finally, the Level 3 system must find a central track of $p_{T}>10 \mathrm{GeV} / c$.

MUW_W_L2M3_TRK10: This trigger requires a Level 1 muon with both scintillator and drift chamber hits. The Level 2 muon is required to have a transverse momentum of $3 \mathrm{GeV} / c$. Otherwise, this is identical to the MU_W_L2M5_TRK10.

MUW_A_L2M3_TRK10: The trigger is the same as MU_W_L2M3_TRK10, 
except that Level 1 muons outside of the CFT acceptance are used as well.

It should be noted that the Level 3 tracking algorithm requires a hit in one of the outer two layers of the fiber tracker. Thus there is always an implicit trigger requirement of $|\eta|<1.5$ on the muon (although this may be satisfied by any track in the event). Of the events passing the trigger and data quality requirements, $2,081,825$ have a loose quality muon of $p_{T}>20 \mathrm{GeV} / c$ with a central track match. This is the data set used for the Technicolor search.

Signal samples were generated with PYTHIA 6.224 [13], [45]. The CTEQ5L parton distribution functions were used [47]. A grid of twenty hypothetical $\rho_{T}-\pi_{T}$ mass combinations were generated, shown in Figure 6.1, along with the associated cross sections. Table 6.1 summarizes the same information.

Backgrounds to the $\rho_{T} \rightarrow W \pi_{T} \rightarrow \mu \nu b \bar{b} / b \bar{c}$ production includes processes which mimic the $\mu \nu b j$ final state topology (including dimuon processes, in which one muon is not reconstructed); processes that contain a muon from vector boson production, but no $b$-flavored jets (or only charm jets); and non$W / Z$ muons that arise from hadronic decays or the misreconstruction of jet activity (both are sometimes referred to as QCD or instrumental backgrounds).

Estimates of the background expectations and distributions were obtained from simulated data samples, listed (with the sample sizes) in Table 6.2. Inteference from virtual photons was considered for all samples containing a $Z$ execpt the $W Z$ and $Z Z$ channels. For the samples generated with ALPGEN or COMPHEP, PYTHIA was used to simulate the showering and hadronization process. All other samples were generated with PYTHIA. The calculation of the cross sections, with uncertainties, is discussed in Section 6.1.1.

Some requirements were imposed on the generated particles to increase the number of events which survive to the final sample. In the weak boson plus jets (including the $b \bar{b}$ samples), muons were required to have $|\eta|<10$, with a dimuon mass, where applicable, between $50 \mathrm{GeV} / c^{2}$ and $200 \mathrm{GeV} / c^{2}$. Criteria were also imposed on jets reconstructed from partons: $p_{T}>8 \mathrm{GeV} / c$, $|\eta|<3$, with jets separated by $\Delta R>0.4$. In the $W \rightarrow \mu \nu / \tau \nu$ and $Z \rightarrow \mu \mu / \tau \tau$ samples, the leptons were limited to the region $|\eta|<4.2$ [51]. Finally, samples were generated for each of the $Z \rightarrow \mu \mu$ and $Z \rightarrow \tau \tau$ channels with two allowed ranges of the dilepton mass $\left(15-60 \mathrm{GeV} / c^{2}\right.$ and $\left.60-130 \mathrm{GeV} / c^{2}\right)$. Note that prior to the application MLM matching scheme [50], any number of jets was allowed in the $W$ and $Z$ samples.

To good approximation, the kinematic distributions for the $W Z \rightarrow \mu \nu c \bar{c} / j j$ and $Z Z \rightarrow \mu \nu c \bar{c} / j j$ channels are the same as those in the existing diboson samples. Therefore, the corresponding acceptances are estimated by re- 

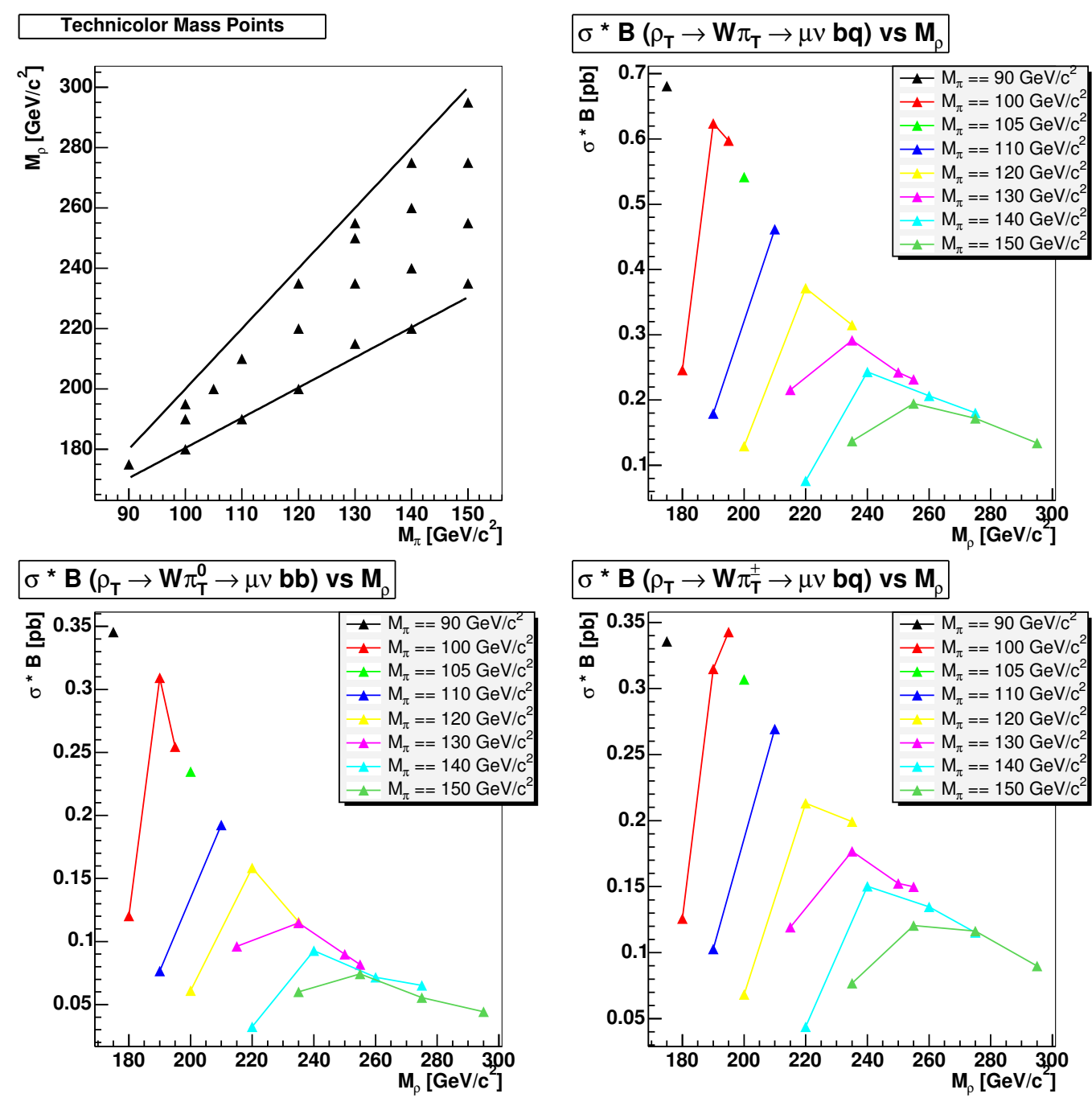

Figure 6.1: Grid of generated Technicolor mass hypotheses (top left) and cross sections times branching ratios summed over all charge states (top right) and for the individual the $\rho_{T}^{0} \rightarrow W^{ \pm} \pi_{T}^{0}$ (bottom left) $\rho_{T}^{0} \rightarrow W^{ \pm} \pi_{T}^{\mp}$ (bottom right) states. All cross sections are for $M_{V}=100 \mathrm{GeV}$. The upper line in the mass grid indicates the threshold above which $\rho_{T} \rightarrow \pi_{T} \pi_{T}$ becomes kinematically allowed (and preferred). Below the lower line, $M_{\rho}<M_{W}+M_{\pi}$, the $\rho_{T} \rightarrow W \pi_{T}$ decay channel is suppressed. 


\begin{tabular}{|rr|rr|rr|}
\hline & & \multicolumn{2}{|c|}{$\rho_{T} \rightarrow W \pi_{T}^{0} \rightarrow \mu \nu b b$} & $\rho_{T} \rightarrow W \pi_{T}^{ \pm} \rightarrow \mu \nu b c$ \\
\hline$M_{\rho}\left(\mathrm{GeV} / c^{2}\right)$ & $M_{\pi}\left(\mathrm{GeV} / c^{2}\right)$ & $\sigma \times B(p b)$ & Events & $\sigma \times B(p b)$ & Events \\
\hline 175 & 90 & 0.35 & 15,000 & 0.34 & 15,000 \\
180 & 100 & 0.12 & 15,000 & 0.13 & 15,000 \\
190 & 100 & 0.31 & 21,000 & 0.31 & 20,000 \\
190 & 110 & 0.08 & 15,000 & 0.10 & 15,000 \\
195 & 100 & 0.25 & 15,000 & 0.34 & 15,000 \\
200 & 105 & 0.23 & 31,250 & 0.31 & 21,000 \\
200 & 120 & 0.06 & 15,000 & 0.07 & 15,000 \\
210 & 110 & 0.19 & 26,250 & 0.27 & 35,500 \\
215 & 130 & 0.10 & 15,000 & 0.12 & 15,000 \\
220 & 120 & 0.16 & 15,000 & 0.21 & 15,000 \\
220 & 140 & 0.03 & 15,000 & 0.04 & 15,000 \\
235 & 120 & 0.12 & 15,000 & 0.20 & 15,000 \\
235 & 130 & 0.11 & 15,000 & 0.18 & 15,000 \\
235 & 150 & 0.06 & 15,000 & 0.08 & 15,000 \\
240 & 140 & 0.09 & 15,000 & 0.15 & 15,000 \\
250 & 130 & 0.09 & 20,250 & 0.15 & 32,000 \\
255 & 130 & 0.08 & 15,000 & 0.15 & 15,000 \\
255 & 150 & 0.07 & 15,000 & 0.12 & 15,000 \\
260 & 140 & 0.07 & 15,000 & 0.13 & 15,000 \\
275 & 140 & 0.06 & 15,000 & 0.12 & 15,000 \\
\hline
\end{tabular}

Table 6.1: Cross sections times branching fractions and total events in generated Technicolor samples, separated by charge state. Cross sections are for $M_{V}=100 \mathrm{GeV}$. 


\begin{tabular}{|l|ll|}
\hline Process & $\sigma \times B(p b)$ & Events \\
\hline$W \rightarrow \mu \nu$ & $2684 \pm 134$ & $1,065,750$ \\
$W \rightarrow \tau \nu$ & $2684 \pm 134$ & 880,792 \\
$Z / \gamma^{*} \rightarrow \mu \mu(60-130)$ & $246 \pm 12$ & 506,250 \\
$Z / \gamma^{*} \rightarrow \tau \tau(60-130)$ & $246 \pm 12$ & 403,000 \\
$Z \rightarrow \mu \mu(15-60)$ & $528 \pm 26$ & 312,750 \\
$Z \rightarrow \tau \tau(15-60)$ & $528 \pm 26$ & 503,250 \\
$W j j \rightarrow \mu \nu j j$ & $407 \pm 65$ & 188,000 \\
$Z j j \rightarrow \mu \mu j j$ & $42.9 \pm 6.9$ & 188,000 \\
$W W \rightarrow l \nu j j$ & $5.80 \pm 0.58$ & 23,000 \\
$W Z \rightarrow j j l l$ & $0.236 \pm 0.024$ & 25,000 \\
$W Z \rightarrow \mu \nu b b$ & $0.054 \pm 0.005$ & 39,500 \\
$Z Z \rightarrow \mu \mu b b$ & $0.019 \pm 0.002$ & 46,000 \\
$t \bar{t} \rightarrow l \nu j j b \bar{b}$ & $2.94 \pm 0.53$ & 45,750 \\
$t \bar{t} \rightarrow l \nu l \nu b \bar{b}$ & $0.70 \pm 0.13$ & 48,500 \\
$q t b \rightarrow q \mu \nu b \bar{b}$ & $0.258 \pm 0.039$ & 15,500 \\
$W^{*} \rightarrow t b \rightarrow \mu \nu b \bar{b}$ & $0.115 \pm 0.018$ & 29,000 \\
$W b b \rightarrow \mu \nu b b$ & $2.57 \pm 0.36$ & 84,000 \\
$Z b b \rightarrow \mu \mu b b$ & $0.469 \pm 0.075$ & 96,500 \\
\hline
\end{tabular}

Table 6.2: Simulated background samples. The $Z / \gamma^{*}$ channels are split into two dilepton mass ranges $\left(15 \mathrm{GeV} / c^{2}<M_{l l}<60 \mathrm{GeV} / c^{2}\right.$ and $\left.60 \mathrm{GeV} / c^{2}<M_{l l}<130 \mathrm{GeV} / c^{2}\right)$. 


\begin{tabular}{lll}
\hline Process & $\sigma \times B(p b)$ & Analyzed sample \\
\hline$W Z \rightarrow \mu \nu j j$ & $0.154 \pm 0.015$ & $W Z \rightarrow \mu \nu b \bar{b}$ \\
$W Z \rightarrow \mu \nu c \bar{c}$ & $0.0418 \pm 0.0042$ & $W Z \rightarrow \mu \nu b \bar{b}$ \\
$Z Z \rightarrow \mu \mu j j$ & $0.0541 \pm 0.0054$ & $W Z \rightarrow \mu \mu b \bar{b}$ \\
$Z Z \rightarrow \mu \mu c \bar{c}$ & $0.0147 \pm 0.0015$ & $W Z \rightarrow \mu \mu b \bar{b}$ \\
\hline
\end{tabular}

Table 6.3: Diboson backgrounds without a separate simulated sample. Also shown are the cross sections times branching fractions and the actual samples analyzed.

analyzing the $W Z \rightarrow \mu \nu b \bar{b}$ and $Z Z \rightarrow \mu \nu b \bar{b}$ while treating $b$-jets as charm or light jets when evaluating the tag rate functions. ${ }^{1}$ Table 6.3 lists the cross sections and actual sample analyzed for these channels.

\subsubsection{Simulated Cross Sections}

Signal cross sections were calculated at leading order with the same version of PYTHIA used in the event generation. Next to leading order (NLO) corrections were approximated through use of a K-factor, that multiplies the leading order value:

$$
K=1+\frac{\alpha_{S}}{\pi} \frac{2}{3}\left(1+\frac{4}{3} \pi^{2}\right)
$$

The K-factor depends on the momentum transfer, $q^{2}$, of the collision through the QCD interaction strength, $\alpha_{S}$. However, for $q^{2} \approx M_{\rho}^{2}, K$ may be considered to have a constant value of 1.3 [43].

Most background cross sections are measured with MCFM [46], [58]. Conservatively, the systematic uncertainities for all background cross sections were assumed to be $100 \%$ correlated. Uncertainties of $5 \%$ are assigned for inclusive $W$ and $Z$ production, based on the size of the next to next to leading order (NNLO) corrections to the theoretical prediction [59].

The diboson cross sections were estimated by multiplying the $W Z \rightarrow \mu \nu e e$ and $Z Z \rightarrow \mu \mu e e$ figures from MCFM [60] by the PDG ratio of branching

\footnotetext{
${ }^{1}$ Because of problems during the generation phase, no samples were available for these channels. Hence the contributions for these channels (expected to be small) were estimated from existing samples. A $10 \%$ systematic was assigned to the results, to allow for differing kinematic acceptances. This was estimated by examining small $W Z \rightarrow \mu \nu c \bar{c} / j j$ that were available.
} 
fractions: $B(Z \rightarrow b \bar{b}) / B(Z \rightarrow e e), B(Z \rightarrow c \bar{c}) / B(Z \rightarrow e e)$, etc [2]. The $W W$ cross sections were taken from Reference [61]. An uncertainty for each is determined to be $10 \%$ from variations of the cross sections with the changes in the renormalization scale.

The $W / Z j j$ cross sections were determined by demanding that accepted cross section for requiring two parton jets with $p_{T}>25 \mathrm{GeV} / c$ and $|\eta|<2.5$ agrees with the MCFM prediction for the same region of phase space. This was done because PYTHIA may not describe the showering as well as MCFM, an effect that could lead to differing jet momentum distributions. This phase space matching technique forces the correct cross section for events likely to pass the selection criteria [51]. Uncertainties of $16 \%$ were assigned, based on scale variations.

Likewise, the $W b \bar{b}$ and $Z b \bar{b}$ cross sections were also evaluated by phase space matching with MCFM. The matching region was more tightly constrained, however, also requiring a dijet mass of at least $70 \mathrm{GeV} / c^{2}$. MCFM tends to overestimate the cross section for low jet-jet masses because of a massless $b$-quark approximation [51]. The assigned uncertainties were $14 \%$ for $W b \bar{b}$ production and $16 \%$ for the $Z b \bar{b}$ channel, again taken from scale variations.

Finally the top production cross sections were assumed to be $6.77 \pm 0.42$ $\mathrm{pb}$ for $t \bar{t}[62] ; 1.98 \pm 0.13 \mathrm{pb}$ for $q t b$ and $0.88 \pm 0.13 \mathrm{pb}$ for $W^{*} \rightarrow t b$ [63]. A top mass of $175 \mathrm{GeV} / c^{2}$ was assumed. Including uncertainties in $m_{t}$, the total uncertainties for these cross sections are $18 \%, 15 \%$ and $16 \%$ respectively. Finally, a branching fraction of 0.13041 was used for $W \rightarrow \mu \nu$ in the single-top channels. ${ }^{2}$

\subsection{Selection of the $W b j \rightarrow \mu \nu b j$ Event Sample}

Events are selected that are consistent with $W \rightarrow \mu \nu$ plus heavy flavor jets production:

- There must be at least one primary vertex with $|z|<60 \mathrm{~cm}$ (i.e., in the SMT acceptance) and be associated with at least three tracks.

- The event must have an isolated medium quality muon matched to a central track with $p_{T}>20 \mathrm{GeV} / \mathrm{c}$.

- The missing $E_{T}$ in the event must be at least $20 \mathrm{GeV}$.

\footnotetext{
${ }^{2}$ This differs from the usual rate $B(W \rightarrow \mu \nu)=0.1049$ because it includes the rate for leptonic $\tau$ decays.
} 
- The transverse mass, $M_{T}$, of the muon and the missing $E_{T}$ must be greater than $30 \mathrm{GeV} / c^{2}$.

- The event must have at least two jets with $p_{T}>20 \mathrm{GeV} / c,|\eta|<2.5$.

- At least one of the two leading jets must be tagged with the JLIP algo$\operatorname{rithm}\left(\mathcal{P}_{\text {jet }}<0.3 \%\right)$.

- Events with a third jet are rejected if it is taggable and has $p_{T}>$ $15 \mathrm{GeV} / c$.

- Events with an electron or an additional muon with $p_{T}>10 \mathrm{GeV} / c$ are rejected.

Details of the object identification requirements were presented in Chapters 3 and 5. The veto on the presence of additional jets or leptons reduces the contributions from the $t \bar{t}$ and $Z$ channels. The transverse mass of the muonmissing $E_{T}$ system is defined as:

$$
M_{T} \equiv \sqrt{2 p_{T}^{\mu} E_{T}(1-\cos \Delta \phi)}
$$

where $p_{T}^{\mu}$ is the transverse momentum of the muon, and $\Delta \phi$ is the azimuthal opening angle between the muon and the missing $E_{T}$. Veto muons must be of medium quality with a central track match and be separated from any jet by a distance, $\Delta R>0.5$. The strict quality requirements detailed in Section 3.4 are applied to the primary muon, but not to the veto muon.

As discussed in Chapter 5, the JLIP tagger is not explicitly run on simulated events. Instead the flavor of a jet, $i$, is determined by matching the generated partons to the reconstructed jets. The appropriate TRF for the jet flavor is evaluated, giving a tagging probability $\epsilon_{i}$. The probability to tag at least one of the two leading jets $(i=1,2)$ is determined:

$$
w=1-\left(1-\epsilon_{1}\right)\left(1-\epsilon_{2}\right)
$$

and used as a weight to fill histograms and calculate the total event yields. Tables 6.4 and 6.5 indicate how the event yields vary as each selection requirement is applied in turn. Also shown are the results of the $L_{T}$ and dijet $p_{T}$ requirements discussed in Section 6.4.

Figures $6.2-6.4$ show the properties of the muon-missing $E_{T}$ system as they evolve with the selection requirements. ${ }^{3}$ The transverse mass distri-

\footnotetext{
${ }^{3}$ Note that unless otherwise specified, all Technicolor distributions shown are for the mass combination $M_{\rho}=200 \mathrm{GeV} / c^{2}, M_{\pi}=105 \mathrm{GeV} / c^{2}$, with $M_{V}=100 \mathrm{GeV}$.
} 

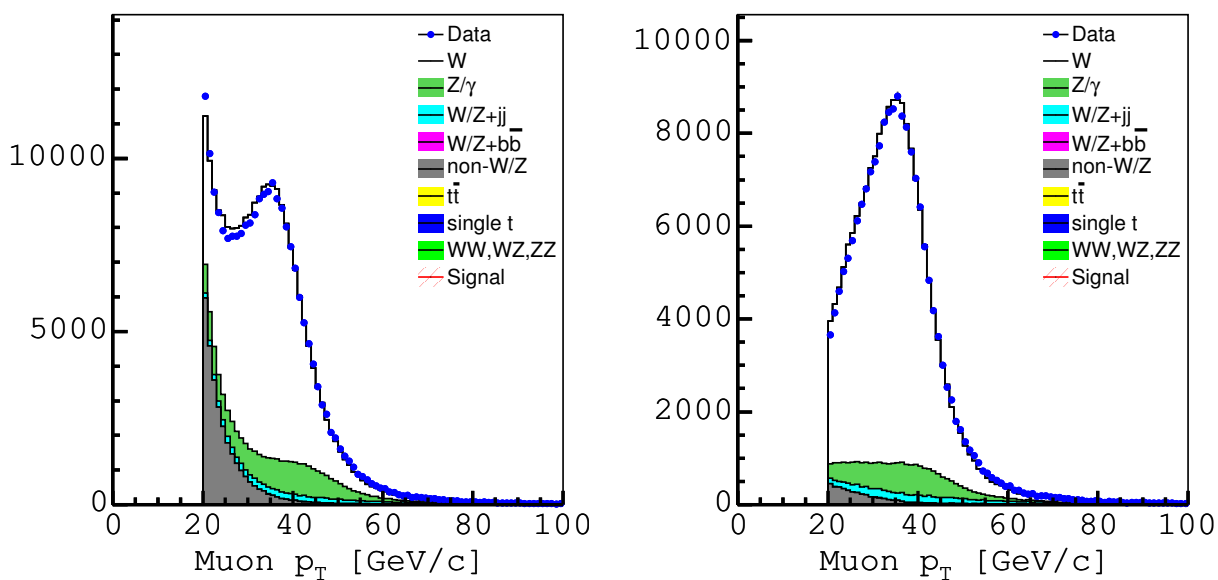

Figure 6.2: Muon $p_{T}$ spectra. The left plot shows the transverse momentum of the leading muon after requiring an isolated muon satisfying all of muon quality criteria and $p_{T}>20 \mathrm{GeV} / c$. The right plot shows the distribution after the $\mathbb{E}_{T}$ requirement has been imposed as well..

bution after the $W$ plus one jet selection exhibits a significant disagreement between data and background expectations on the low mass shoulder of the peak. Because the non- $W / Z$ background peaks in this region, it is hypothesized that the discrepancy is due to an overestimation of this contribution, probably through the use of an incorrect control sample. The issue has resisted all investigations. However, the same distribution at later stages is well behaved (within the available statistics) and the only other anomaly (see Figure 6.8) appears to be a statistical fluctuation. Therefore it is believed to have a negligible impact on the analysis. Nonetheless, it is partly because of this problem that a 100\% systematic uncertainty is assigned to the instrumental backgrounds in Section 6.3.

The jet multiplicity distribution is shown in Figure 6.5. Properties of the leading two jets, and of the dijet system are shown in Figures 6.6 - 6.8. It has been observed that the dijet invariant mass spectrum exhibits a significant deficit near $90 \mathrm{GeV} / c^{2}$. This feature has been investigated and cross-checked with a Standard Model Higgs search in the $W H \rightarrow \mu \nu b \bar{b}$ channel. All evidence strongly indicates that the deficit is a statistical fluctation. In particular, most of the events in the problematic bin are lost to the strict muon quality requirements. 


\begin{tabular}{|l|lllllll|}
\hline sample & $W$ sel. & Dijet sel. & $b$-tag & $3^{\text {rd }}$ jet & $2^{\text {nd }}$ lep & $L_{T}$ & $p_{T}^{j j}$ \\
\hline \hline$W \rightarrow \mu \nu$ & 150000 & 150 & 3.4 & 3.3 & 3.3 & 3.3 & 3.3 \\
$W \rightarrow \tau \nu$ & 4700 & 3.1 & 0.080 & 0.080 & 0.080 & 0.080 & 0.080 \\
$Z / \gamma^{*} \rightarrow \mu \mu(60-130)$ & 16000 & 32 & 0.41 & 0.34 & 0.30 & 0.30 & 0.29 \\
$Z / \gamma^{*} \rightarrow \tau \tau(60-130)$ & 440 & 7.7 & 0.050 & 0.040 & 0.040 & 0.030 & 0.030 \\
$Z / \gamma^{*} \rightarrow \mu \mu(60-130)$ & 710 & 1.7 & 0.010 & 0.010 & 0 & 0 & 0 \\
$Z / \gamma^{*} \rightarrow \tau \tau(60-130)$ & 1.5 & & & & & & \\
$W j j \rightarrow \mu \nu j j$ & 5700 & 2300 & 48 & 35 & 35 & 34 & 29 \\
$Z j j \rightarrow \mu \mu j j$ & 800 & 340 & 7.3 & 5.3 & 3.0 & 2.9 & 2.2 \\
$W W \rightarrow l \nu j j$ & 130 & 61 & 2.7 & 2.4 & 2.4 & 2.4 & 2.0 \\
$W Z \rightarrow \mu \nu j j$ & 9.1 & 4.5 & 0.030 & 0.020 & 0.020 & 0.020 & 0.020 \\
$W Z \rightarrow j j l l$ & 7.2 & 3.5 & 0.14 & 0.13 & 0.070 & 0.070 & 0.050 \\
$W Z \rightarrow \mu \nu c \bar{c}$ & 2.5 & 1.2 & 0.17 & 0.14 & 0.14 & 0.13 & 0.11 \\
$W Z \rightarrow \mu \nu b \bar{b}$ & 3.2 & 1.6 & 0.79 & 0.62 & 0.62 & 0.61 & 0.52 \\
$Z Z \rightarrow \mu \mu j j$ & 4.1 & 2.2 & 0.010 & 0.010 & 0.010 & 0.010 & 0 \\
$Z Z \rightarrow \mu \mu c \bar{c}$ & 1.1 & 0.59 & 0.090 & 0.070 & 0.040 & 0.040 & 0.030 \\
$Z Z \rightarrow \mu \mu b \bar{b}$ & 1.4 & 0.76 & 0.39 & 0.31 & 0.17 & 0.17 & 0.14 \\
$t \bar{t} \rightarrow l \nu j j b \bar{b}$ & 61 & 59 & 29 & 5.6 & 5.3 & 5.3 & 3.2 \\
$t \bar{t} \rightarrow l \nu l \nu b \bar{b}$ & 28 & 21 & 13 & 9.7 & 6.8 & 6.8 & 4.3 \\
$q t b \rightarrow q \mu \nu b \bar{b}$ & 13 & 8.7 & 3.5 & 2.2 & 2.2 & 2.2 & 1.8 \\
$W * \rightarrow t b \rightarrow \mu \nu b \bar{b}$ & 6.1 & 3.9 & 2.4 & 1.9 & 1.9 & 1.8 & 1.4 \\
$W b \bar{b} \rightarrow \mu \nu b \bar{b}$ & 55 & 19 & 9.7 & 8.0 & 8.0 & 7.9 & 6.8 \\
$Z b \bar{b} \rightarrow \mu \mu b \bar{b}$ & 14 & 5.4 & 2.8 & 2.2 & 1.2 & 1.2 & 1.0 \\
non-W/Z & 3900 & 280 & 15 & 11 & 9.9 & 6.8 & 5.8 \\
\hline Total Background & 180000 & 3300 & 140 & 88 & 80 & 76 & 62 \\
\hline Data & 183548 & 2995 & 118 & 73 & 65 & 64 & 52 \\
\hline \hline
\end{tabular}

Table 6.4: Evolution, in collider data and Standard Model expectations, of event yields with the initial selection requirements. Empty fields indicate cases where no events in the simulated sample survive. Uncertainties are suppressed for brevity. 


\begin{tabular}{|ll|lllllll|}
\hline$M_{\rho}\left(\mathrm{GeV} / c^{2}\right)$ & $M_{\pi}\left(\mathrm{GeV} / c^{2}\right)$ & $W$ sel. & Dijet sel. & $b$-tag & $3^{\text {rd }}$ jet & $2^{\text {nd }}$ lep & $L_{T}$ & $p_{T}^{j j}$ \\
\hline \hline 175 & 90 & 40 & 20 & 8.9 & 7.4 & 7.4 & 7.2 & 7.1 \\
180 & 100 & 13 & 7.5 & 3.3 & 2.7 & 2.7 & 2.5 & 2.5 \\
190 & 100 & 38 & 22 & 9.7 & 7.8 & 7.8 & 7.6 & 7.6 \\
190 & 110 & 9.3 & 6.0 & 2.7 & 2.1 & 2.1 & 2.0 & 2.0 \\
195 & 100 & 37 & 21 & 9.2 & 7.4 & 7.3 & 7.2 & 7.1 \\
200 & 105 & 33 & 20 & 9.0 & 7.3 & 7.3 & 7.1 & 7.1 \\
200 & 120 & 7.0 & 4.8 & 2.2 & 1.8 & 1.8 & 1.7 & 1.6 \\
210 & 110 & 28 & 18 & 7.9 & 6.2 & 6.1 & 6.0 & 5.9 \\
215 & 130 & 13 & 9.2 & 4.4 & 3.4 & 3.4 & 3.3 & 3.3 \\
220 & 120 & 23 & 16 & 7.2 & 5.6 & 5.6 & 5.4 & 5.4 \\
220 & 140 & 4.2 & 3.2 & 1.5 & 1.1 & 1.1 & 1.1 & 1.1 \\
235 & 120 & 19 & 13 & 5.9 & 4.5 & 4.5 & 4.5 & 4.3 \\
235 & 130 & 18 & 13 & 6.0 & 4.6 & 4.6 & 4.5 & 4.5 \\
235 & 150 & 8.1 & 6.3 & 3.1 & 2.3 & 2.3 & 2.2 & 2.2 \\
240 & 140 & 15 & 11 & 5.2 & 3.9 & 3.9 & 3.8 & 3.8 \\
250 & 130 & 15 & 11 & 5.0 & 3.8 & 3.8 & 3.8 & 3.6 \\
255 & 130 & 14 & 10 & 4.8 & 3.6 & 3.6 & 3.5 & 3.3 \\
255 & 150 & 12 & 9.3 & 4.5 & 3.3 & 3.3 & 3.2 & 3.2 \\
260 & 140 & 13 & 9.5 & 4.5 & 3.3 & 3.3 & 3.3 & 3.1 \\
275 & 150 & 10 & 8.1 & 3.9 & 2.8 & 2.8 & 2.8 & 2.6 \\
\hline
\end{tabular}

Table 6.5: Evolution of event yields with the initial selection requirements for all twenty Technicolor mass hypotheses, for $M_{V}=100 \mathrm{GeV}$. 

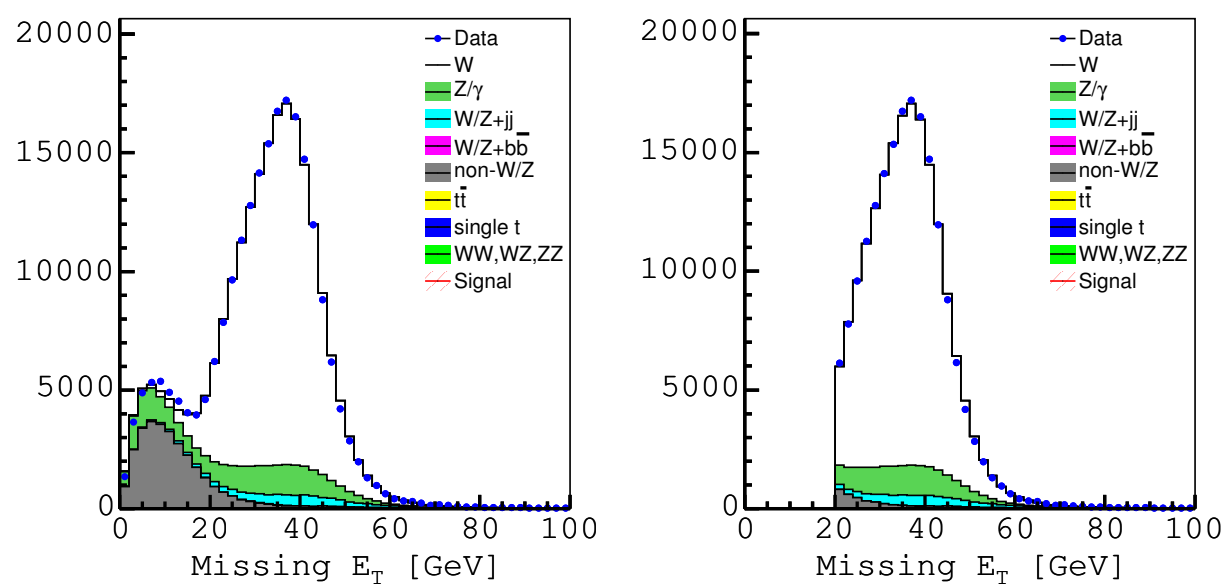

Figure 6.3: Missing $E_{T}$ distributions after muon (left) and after the full W selection criteria (right).

\subsubsection{Normalization Procedure}

The recorded luminosity is not used in this analysis. This is done to remove the contribution of systematic unertainties from the luminosity measurement, the trigger efficiency, and the initial selection requirements. Instead an effective luminosity, $\mathcal{L}^{\prime}$, is calculated by forcing the expected event yield at an early stage of the selection to agree with the collider data.

After imposing the trigger, primary vertex, muon, $\mathbb{E}_{T}$, and transverse mass selection requirements, the number of events, $N_{\text {data }}^{W}$ (observed in data) is

$$
N_{\text {data }}^{W}=\mathcal{L} \sum_{i} \epsilon_{i}^{W, \text { data }} \sigma_{i}
$$

where $\mathcal{L}$ is the recorded luminosity, $\epsilon_{i}^{W, d a t a}$ is the efficiencies for the $i$ 'th process to pass the $W$ selection (and trigger) requirements, and $\sigma_{i}$ is the cross section for that process. The expected number of events, $N_{m c}^{W}$, is

$$
N_{m c}^{W}=\mathcal{L}^{\prime} \sum_{i} \epsilon_{i}^{W, m c} \sigma_{i}
$$

Here, $\epsilon_{i}^{W, m c}$ is the efficiency, measured in simulated data, for selecting events from a particular physics process. The effective luminosity, $\mathcal{L}^{\prime}$, is determined by demanding that $N_{m c}^{W}=N_{W}^{d a t a}$. Therefore 

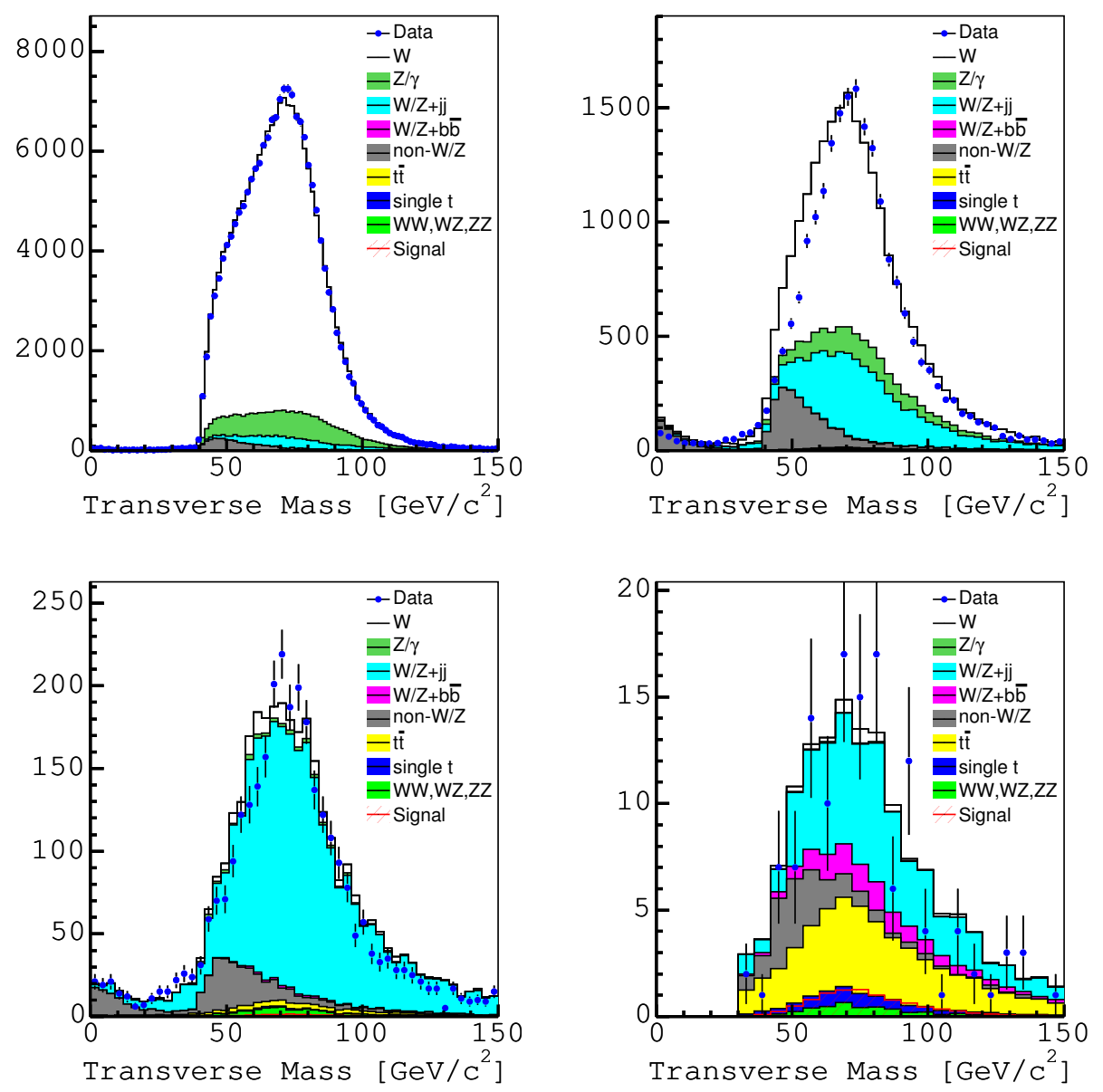

Figure 6.4: Transverse mass of the muon and missing $E_{T}$ after the muon and $E_{T}$ requirements (top left), the additional requirement of at least one (top right) or two (bottom left) jets and the requirement of at least one JLIP tag (bottom right). The Technicolor mass mass combination shown is $M_{\rho}=200 \mathrm{GeV} / c^{2}, M_{\pi}=105 \mathrm{GeV} / c^{2}$, with $M_{V}=100 \mathrm{GeV}$. 

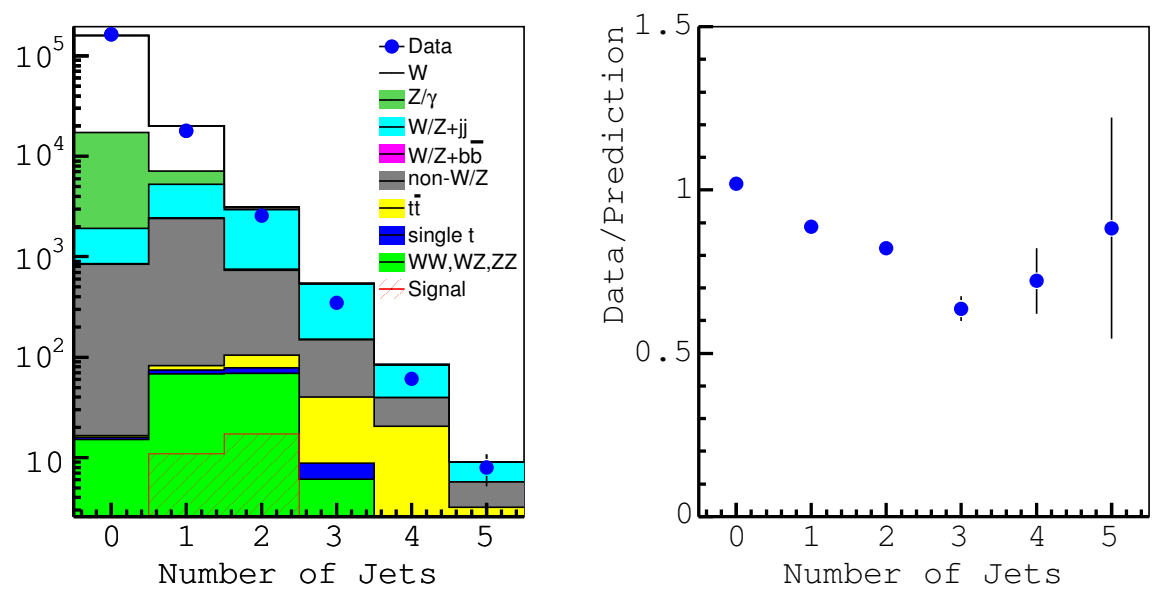

Figure 6.5: Number of jets (left) with $p_{T}>20 \mathrm{GeV} / c$ after the $W$ selection requirements. The right plot gives the ratio of the collider data to the Standard Model prediction. Uncertainties are statistical only

$$
\mathcal{L}^{\prime}=\frac{N_{\text {data }}^{W}}{\sum_{i} \epsilon_{i}^{W, m c} \sigma_{i}}
$$

which differs from the true luminosity by the factor $\sum_{i} \epsilon_{i}^{W, m c} \sigma_{i} / \sum_{i} \epsilon_{i}^{W, \text { data }} \sigma_{i}$, which folds in the trigger efficiencies (not measured for the simulated data) and any scale factors between collider data and simulation for the $W$ selection criteria. After some subsequent set of requirements $\alpha$, the number of expected events for some physics process $j$ is then

$$
N_{j}=\left(\mathcal{L}^{\prime} \epsilon_{j}^{W, m c} \sigma_{i}\right) \epsilon_{j}^{\alpha, M C}
$$

The relative efficiency $\epsilon_{j}^{\alpha, M C}$ of these criteria for each process, $j$, is measured, as is $\epsilon_{i}^{W, M C}$, from the simulation.

This method yields a figure of $201 \pm 10 \mathrm{pb}^{-1}$. As long as the effects absorbed into the effective luminosity do not bias the measurement of $\epsilon_{j}^{\alpha, M C}$, their associated uncertainties do not contribute to the uncertainty of $N_{j}$. Specifically, because the muon trigger efficiency and reconstruction scale factors do not vary with transverse momentum or psuedorapidity, the corresponding systematic uncertainties cancel [51]. Thus, knowledge of $\mathcal{L}^{\prime}$ is primarily limited by the $5 \%$ uncertainty on the inclusive $W$ cross section [59]. 

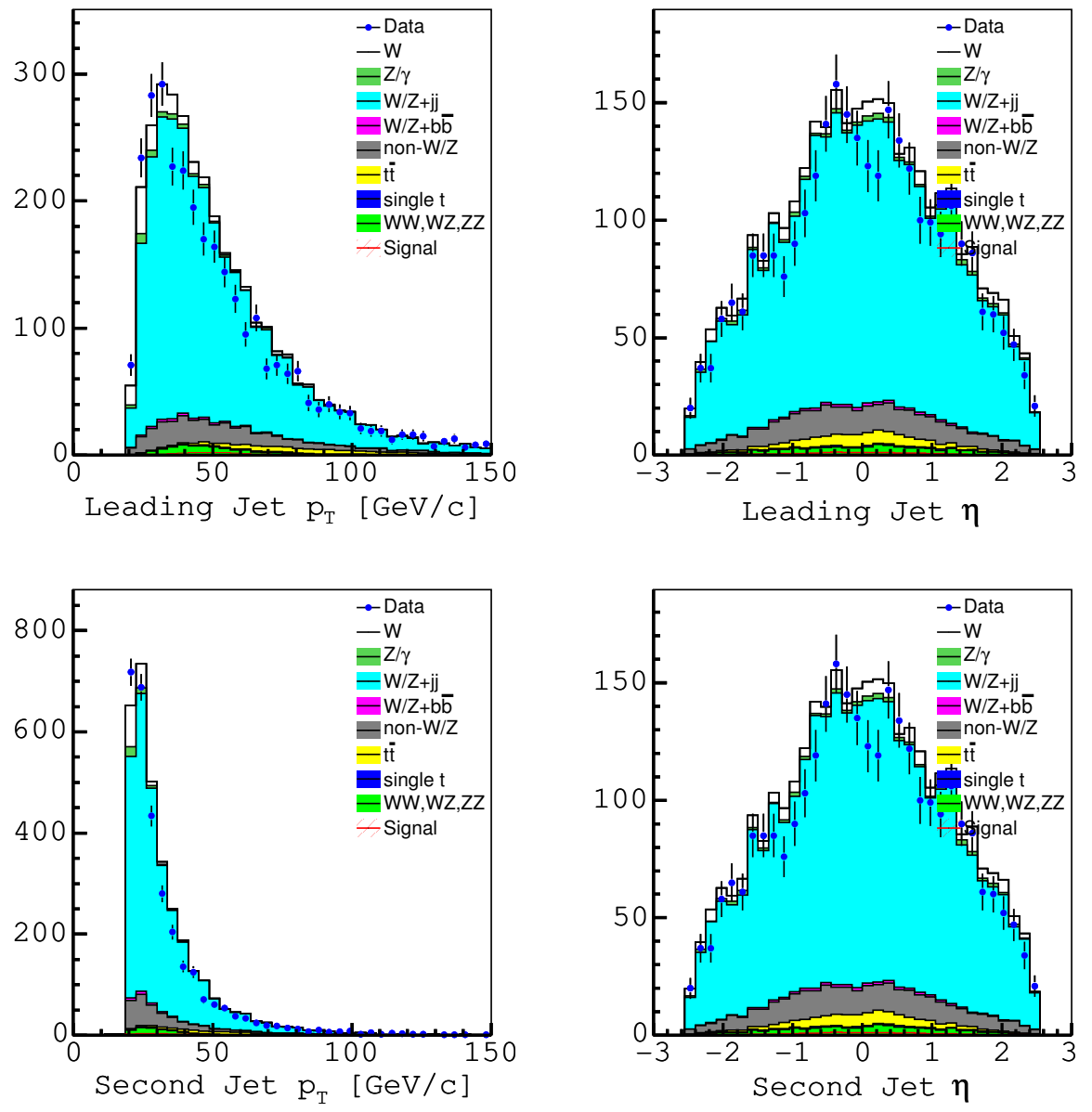

Figure 6.6: Transverse momentum and pseudorapidiy distributions for the two highest energy jets after the $W j j$ selection. No tagging requirement, jet or lepton vetos are yet applied. 

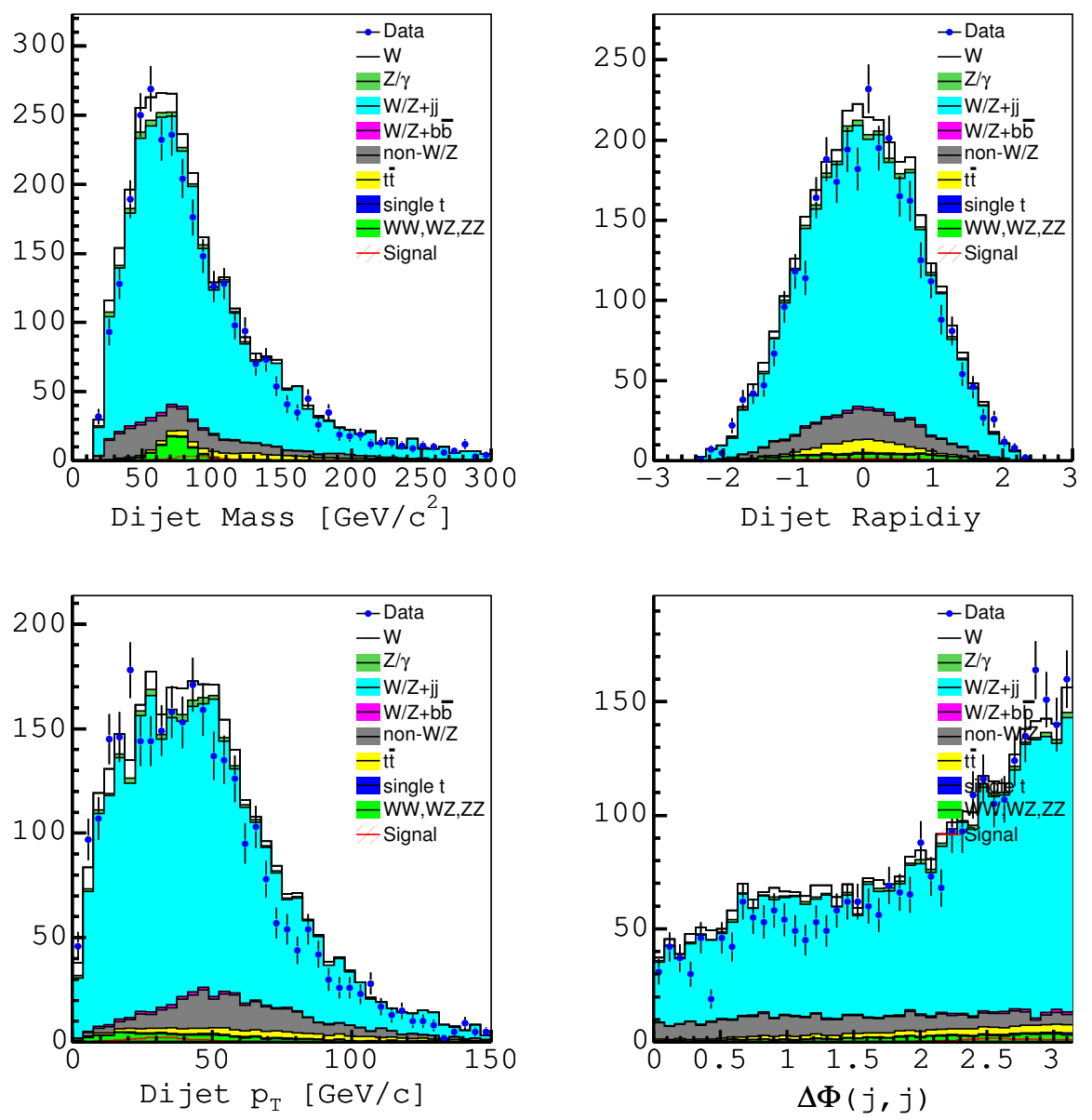

Figure 6.7: Invariant mass (top left), true rapidity (top right) transverse momentum (bottom left) and azimuthal opening angle (bottom right) of the dijet system. Distributions are at the $W j j$ selection stage. 

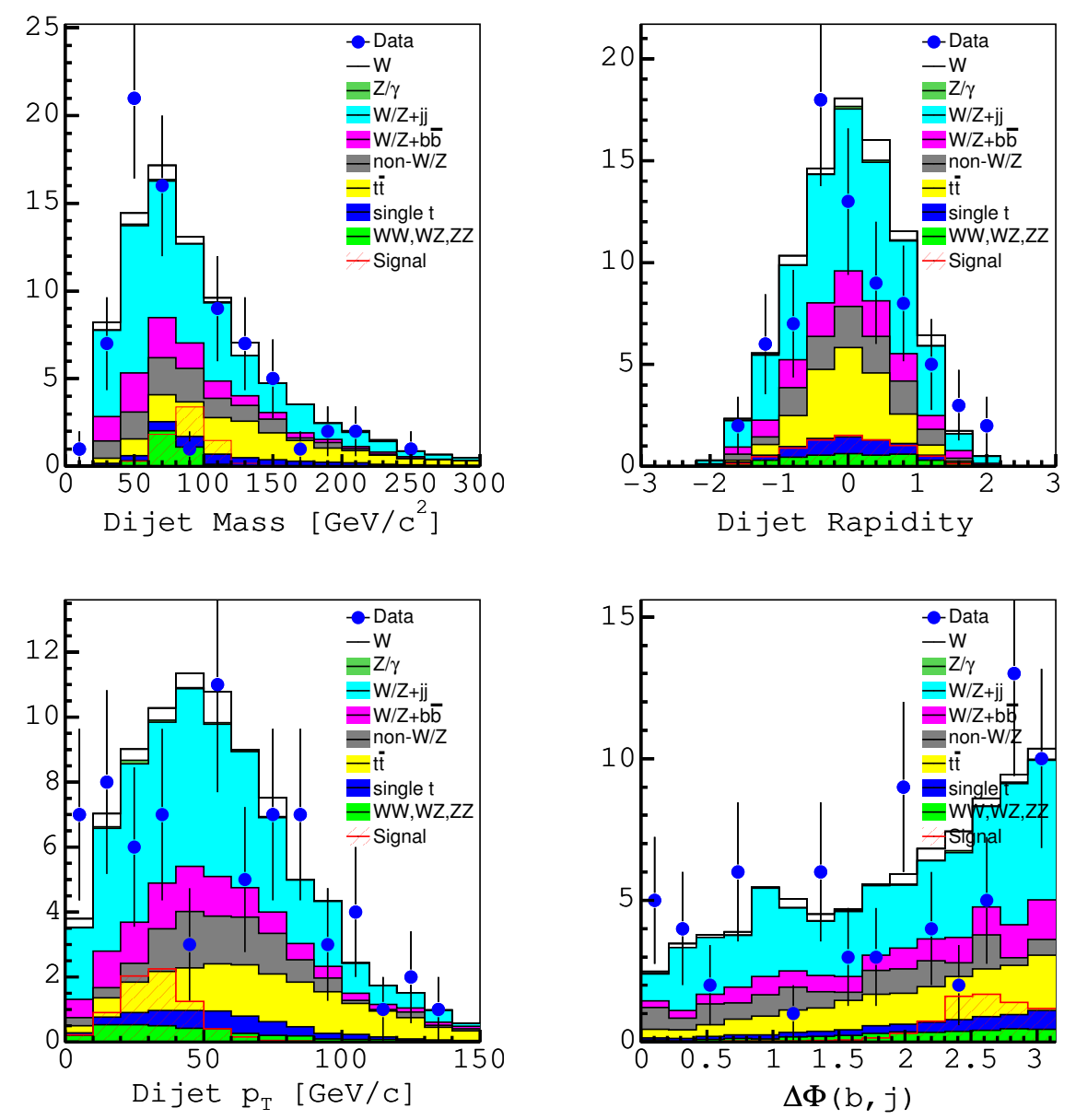

Figure 6.8: Properties of the dijet system, as in Figure 6.7 but after the $b$ tagging, third jet veto and second lepton veto requirements are applied. 


\subsection{Estimation of the Instrumental Background}

Most of the backgrounds considered in this analysis are characterized by isolated muons from real $W$ or $Z$ bosons. But there are additional bakgrounds from other sources. These include real muons from hadronic decays; jets that punch through the calorimeter to the muon system; and charged particles other than muons that occasionally traverse the detector (sail thorugh) without significant showering.

These effects are measured from collider data by first imposing all selection requirements except the muon isolation (which is a powerful tool for removing such backgrounds). The numbers of events in this sample passing and failing the isolation requirement ( $N_{\text {pass }}$ and $N_{\text {fail }}$, respectively) can expressed in terms of the signal, $S$, and the background, $B$, contributions to the parent sample: ${ }^{4}$

$$
\begin{gathered}
N_{\text {pass }}=\epsilon S+f B \\
N_{\text {fail }}=(1-\epsilon) S+(1-f) B
\end{gathered}
$$

where $\epsilon$ is the efficiency for the isolation requirement on signal muons and $f$ is the probability (or fake rate) for a background muon to be falsely isolated. The background contribution after the isolation requirement may therefore be written as

$$
f B=f \frac{\epsilon N_{\text {fail }}-(1-\epsilon) N_{\text {pass }}}{\epsilon-f}
$$

In the case of a variable efficiency or fake rate, the background may be evaluated on an event by event basis and the results summed to give a total estimate of the instrumental background.

To measure $\epsilon$, dimuon events in which both muons satisfy the local and central track criteria detailed in Section 3.4 are selected. The dimuon mass, is further required to be between $75 \mathrm{GeV} / c^{2}$ and $105 \mathrm{GeV} / c^{2}$, giving a rather pure sample of $Z \rightarrow \mu \mu$ decays. The fraction of dimuon events in which exactly one muon is isolated is $2 \epsilon(1-\epsilon)$, and the fraction in which both are isolated is $\epsilon^{2}$. The isolation efficiency is measured in both classes of events. The value of $\epsilon$ is observed to vary with the number of jets, as shown in Table 6.6.

The fake rate is estimated by selecting events with exactly one muon and a missing $E_{T}$ of at most $5 \mathrm{GeV}$. The probability for such muons to be isolated is observed to differ depending on the topology of the event. If no jets are

\footnotetext{
${ }^{4}$ In this case, signal refers to events with muons from $W$ or $Z$ decays, while background indicates only instrumental backgrounds.
} 


\begin{tabular}{llll}
\hline Number of jets & $\geq 0$ & $\geq 1$ & $\geq 2$ \\
\hline single isolated & $0.957 \pm 0.002$ & $0.89 \pm 0.01$ & $0.89 \pm 0.03$ \\
double isolated & $0.956 \pm 0.002$ & $0.89 \pm 0.01$ & $0.89 \pm 0.03$ \\
\hline
\end{tabular}

Table 6.6: Per-muon isolation efficiencies for muons from $\mathrm{Z}$ events as a function of the number of jets in the event. The first row gives the efficiency as measured from events where exactly one muon is isolated. The second row gives the efficiency as measured from events in which both muons are isolated. Note that the two rows are consistent with each other.

required, $f$ is observed to be $0.179 \pm 0.002$. In events with at least one jet, a value of $0.141 \pm 0.002$ is seen. When a second jet is required the fake rate is $0.080_{-0.004}^{+0.005}$. When at least one jet is required to be $b$-tagged and a third jet veto is applied the fake rate is measured to be $0.06 \pm 0.01$ and $0.05 \pm 0.01$ respectively.

During the analysis, the values of $\epsilon$ and $f$ are chosen to match the level of selection at each stage of the analysis. It is not expected that applying any $b$-tagging should change the isolation efficiency and the sample size is too small to make a measurement to confirm this. Therefore $\epsilon$ is assumed not to vary at the later stages of the analysis.

It is unclear whether the exclusive single muon, low $\mathbb{E}_{T}$ sample correctly models the hadronic decay, punch through and sail through backgrounds. It may not contain these contributions in the correct proportions (in particular the sail through component). In turn, this may bias the measurement of $f$. However, it is also unclear how to select an appropriate control sample. Therefore, instead of using the statistical uncertainties on $\epsilon$ and $f$, a systematic uncertainty of $100 \%$ is assigned to the total instrumental background. This also accounts for the discrepancy in the transverse mass distribution at the $W j$ selection stage (the top right plot in Figure 6.4).

\subsection{Final Event Selection}

After the selection requirements described in Section 6.2, the Standard Model expectation is approximately eighty events, whereas at most eight signal events are expected (depending on the assumed masses). Thus, additional selection criteria are needed to improve the sensitivity to Technicolor processes. Five variables were used as discriminants.

The $\rho_{T}$ and $\pi_{T}$ resonances may be exploited by searching for peaks in 


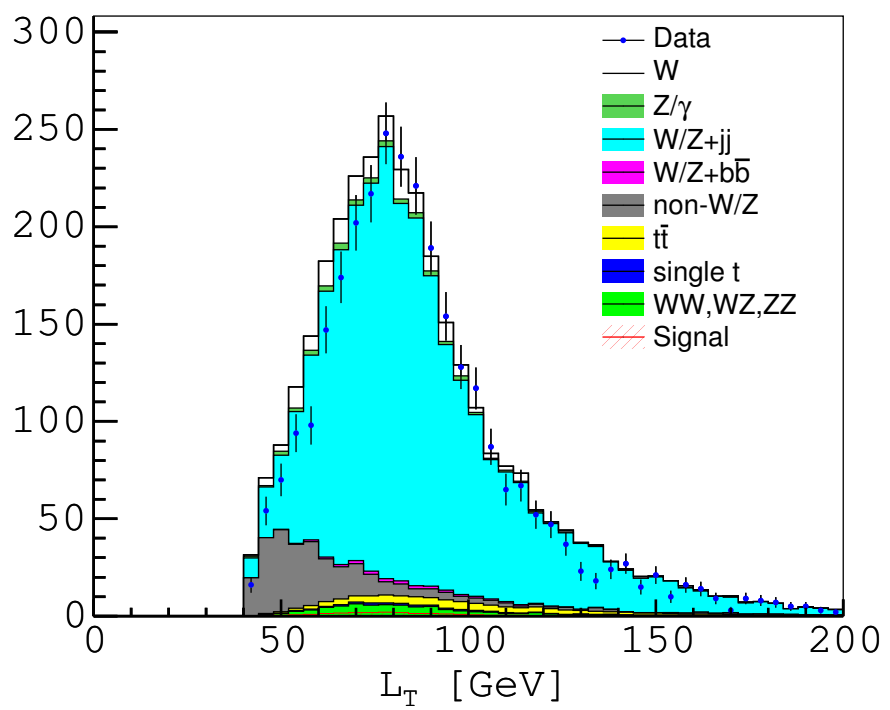

Figure 6.9: Distribution of $L_{T}$ at the $W j j$ selection stage.

the distributions of the dijet mass $\left(m_{j j}\right)$ and mass of the $W$ plus dijet system $\left(m_{W j j}\right.$, also called the four-body mass). This is done by requiring that each mass variable fall within some range (window), that varies with the assumed values of $M_{\pi}$ and $M_{\rho}$. Evaluation of $m_{W j j}$ requires knowledge of the $z$-component of the neutrino momentum, $p_{z}^{\nu}$. Since this cannot, in general, be measured in hadronic collisions, it is estimated by requiring that the muonneutrino system have an invariant mass equal to $M_{W}$. This yields a quadratic equation in $p_{z}^{\nu}$ and the solution with the smaller magnitude is chosen. There is no solution when the measured transverse mass $M_{T}$ is greater than the true $W$ mass. In this case the $\mathscr{E}_{T}$ is rescaled for the purposes of the calculation so that $M_{T}=M_{W}$.

Since heavy particles such as the $\pi_{T}$ tend to be produced at rest, their decay products are normally back to back in the $x-y$ plane. As a result, the azimuthal opening angle between the two jets, $\Delta \phi(j, j)$ may be used to reject backgrounds from non-resonant production of jets. Likewise the total transverse momentum of the dijet system, $p_{T}^{j j}$ will also be small. Finally, the scalar sum, $L_{T}$, of the muon transverse momentum and the missing $E_{T}$ may be used to further suppress instrumental backgrounds by taking advantage of correlations between the two variables. The $L_{T}$ distribution at the $W j j$ selection stage is shown in Figure 6.9. 
Optimal selection criteria were determined for each mass hypothesis by adjusting the requirements on each of the five variables and evaluating the resulting signal $(S)$ and background $(B)$ expectations. As a figure of merit, a modified signal significance, $\mathcal{S}$, is used:

$$
\mathcal{S}=\frac{S}{\sqrt{B+f_{\text {sys }}^{2} B^{2}}}
$$

where $f_{\text {sys }}=30 \%$ is an estimate of the relative systematic uncertainty on the background, obtained from previous iterations on the analysis. The significance thus accounts for the precision with which the background is known, as well as the intrinsic $\mathrm{P}$ oisson width of the estimate, $\sqrt{B}$. It has been found that while inclusion of this term can alter the results significantly (i.e., the choice of the optimal criteria), the sensitivity to the exact value of $f_{\text {sys }}$ is small.

\subsubsection{Optimized Rejection of the Non- $W / Z$ Backgrounds}

In practice, the power of the $L_{T}$ and $p_{T}^{j j}$ variables lies in the rejection of the non- $W / Z$ backgrounds. The method for estimating these contributions requires analysis of events which fail the muon isolation criterion.

The requirements (cuts) on $L_{T}$ and $p_{T}^{j j}$ are optimized separately from the other cuts. This is done by saving the distributions of these variables (after the initial selection detailed in Section 6.2) in two-dimensional histograms. If $h_{s}\left(L_{T}, p_{T}^{j j}\right)$ is the signal histogram and $h_{b}$ is the background histogram, then the expectations for $L_{T}>L_{T}^{\min }$ and $p_{T}^{j j}<p_{T}^{j j, \text { max }}$ may be evaluated as integrals over the distributions:

$$
\begin{aligned}
& S=\int_{L_{T}^{\min }}^{\infty} \int_{0}^{p_{T}^{j j, \max }} h_{s}\left(L_{T}, p_{T}^{j j}\right) d L_{T} d p_{T}^{j j} \\
& B=\int_{L_{T}^{\min }}^{\infty} \int_{0}^{p_{T}^{j j, \max }} h_{b}\left(L_{T}, p_{T}^{j j}\right) d L_{T} d p_{T}^{j j}
\end{aligned}
$$

Strictly, these integrals over distributions are in fact discrete summations over the histograms, but the notation is more cumbersome and obscures the underlying method. Figure 6.10 shows the joint $L_{T}-p_{T}^{j j}$ distributions for background and signal (for $M_{\rho}=200 \mathrm{GeV} / c^{2}, M_{\pi}=105 \mathrm{GeV} / c^{2}$ with $M_{V}=100 \mathrm{GeV}$ ). Optimal cuts are selected by identfiying the values of $L_{T}^{\min }$ and $p_{T}^{j j, \max }$ that maximize $\mathcal{S}$. The optimization is done separately for each mass point and the loosest cut for each variable is chosen, which may not arise from the same 


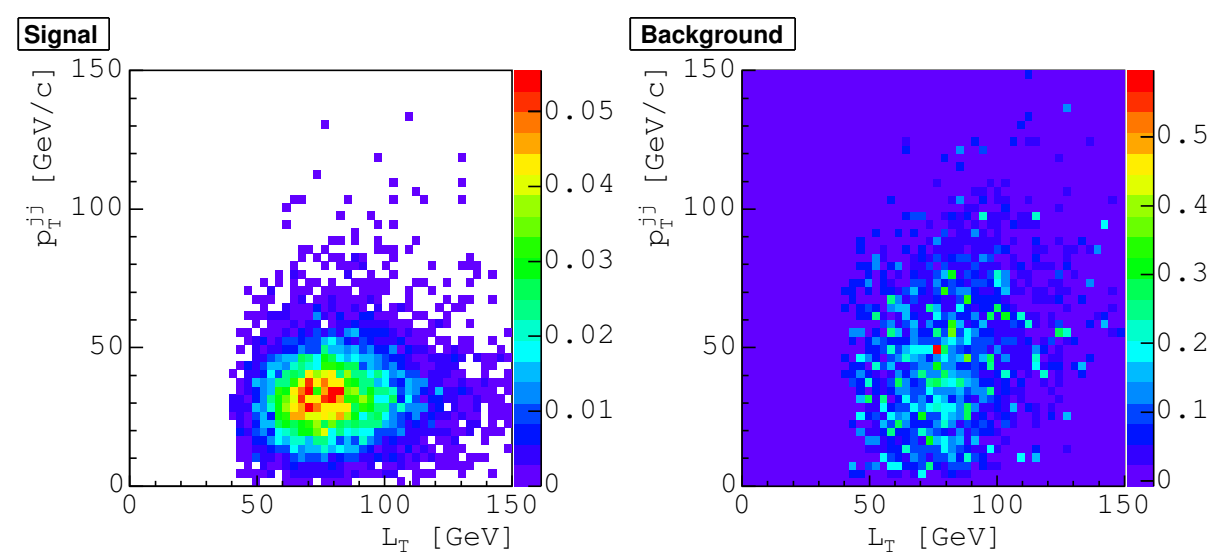

Figure 6.10: Distribution of $p_{T}^{j j}$ versus $L_{T}$ for signal (left) and background (right).

point. Since this procedure involves only a loop over all histogram bins, the computing resources required are very small. However the finite bin width also limits the precison with which optimal cuts can be chosen. This method yields the criteria:

$$
\begin{array}{r}
L_{T}>50 \mathrm{GeV} \\
p_{T}^{j j}<85 \mathrm{GeV} / c
\end{array}
$$

\subsubsection{The Random Grid Search Technique}

Cuts on the remaining variables $\left(m_{j j}, m_{W j j}\right.$ and $\left.\Delta \phi(j, j)\right)$ are chosen separately for each mass hypothesis with a random grid search technique. Each event in the signal samples that passes the initial selection criteria described in Section 6.2 is used to define a cut-point: $m_{j j}^{\min }, m_{j j}^{\max }, m_{W j j}^{\min }, m_{W j j}^{\max }$ and $\Delta \phi(j, j)^{\text {min }}$. The value of $\Delta \phi(j, j)^{\text {min }}$ is the dijet azimuthal opening angle. For the dijet mass requirements, the center of the selection range was set to $m_{j j}$. The full width of the range is chosen to be two standard deviations of the invariant mass distribution (after the initial selection). The same method was used for obtaining the $m_{W j j}$ window.

The signal and background expectations are then calculated for each cutpoint by requiring: 


$$
\begin{array}{r}
m_{j j}^{\min }<m_{j j}<m_{j j}^{\max } \\
m_{W j j}^{\min }<m_{W j j}<m_{W j j}^{\max } \\
\Delta \phi(j, j)>\Delta \phi(j, j)^{\min }
\end{array}
$$

The non- $W / Z$ background yield is not estimated, as the total contribution is small. The cut-point that predicts the largest value of $\mathcal{S}$ is chosen. The result is then refined by varying each threshold by a total of $\pm 20 \%$ in $2 \%$ steps and evaluating $\mathcal{S}$. Each side of the mass windows is varied separately. If a new maximum is found, it is used instead, before refining the next requirement. Figures showing how the significance and selection criteria change with this refinement procedure are contained in Appendix A. The selection requirements chosen, as a function of $M_{\rho}$ and $M_{\pi}$ are shown in Figure 6.11 and Table 6.7. Appendix B summarizes the evolution of signal and background event yields with the application of the final criteria.

As a cross-check, the fractional change in $\mathcal{S}$ after removing the requirement(s) on each variable, but keeping all the others was evaluated. Large values of this marginal signifcance (shown in Figure 6.12) indicate that a selection criterion contributes sensitivity to the search, while small values suggest that the requirement needlessly complicates the analysis. Negative values further imply that the loss of statistical precision actually degrades the sensitivity. Appendix $\mathrm{C}$ shows the distributions of each discriminant variable with the requirement on it removed.

Figure 6.13 shows the final signal and background predictions with full systematic uncertainties, as a function of assumed mass. Also shown are the event yields in collider data. The results, evaluation of the systematic uncertainties and the technique for extracting limits on Technicolor production cross sections are discussed in Chapter 7. 


\begin{tabular}{|cc|ccccc|}
\hline$M_{\pi}$ & $M_{\rho}$ & $M_{j j}^{\min }$ & $M_{j j}^{\max }$ & $M_{W j j}^{\min }$ & $M_{W j j}^{\max }$ & $\Delta \phi(j, j)^{\min }$ \\
\hline 90 & 175 & 64 & 99 & 143 & 182 & 2.52 \\
100 & 180 & 71 & 109 & 153 & 183 & 2.52 \\
100 & 190 & 70 & 108 & 152 & 184 & 2.23 \\
110 & 190 & 79 & 121 & 157 & 220 & 2.58 \\
100 & 195 & 70 & 108 & 153 & 221 & 2.22 \\
105 & 200 & 70 & 109 & 156 & 220 & 2.25 \\
120 & 200 & 91 & 126 & 169 & 206 & 2.54 \\
110 & 210 & 86 & 115 & 170 & 250 & 2.05 \\
130 & 215 & 89 & 142 & 167 & 220 & 2.56 \\
120 & 220 & 91 & 129 & 176 & 237 & 2.09 \\
140 & 220 & 102 & 155 & 179 & 246 & 2.57 \\
120 & 235 & 83 & 123 & 190 & 361 & 1.82 \\
130 & 235 & 97 & 141 & 182 & 250 & 1.93 \\
150 & 235 & 115 & 156 & 195 & 267 & 2.65 \\
140 & 240 & 106 & 142 & 191 & 264 & 2.25 \\
130 & 250 & 98 & 136 & 200 & 365 & 1.78 \\
130 & 255 & 94 & 140 & 205 & 404 & 1.75 \\
150 & 255 & 108 & 158 & 202 & 304 & 2.24 \\
140 & 260 & 107 & 151 & 211 & 396 & 1.88 \\
150 & 275 & 107 & 159 & 217 & 419 & 1.69 \\
\hline
\end{tabular}

Table 6.7: Optimzed selection requirements by assumed Technicolor mass. All masses are in $\mathrm{GeV} / c^{2}$. 

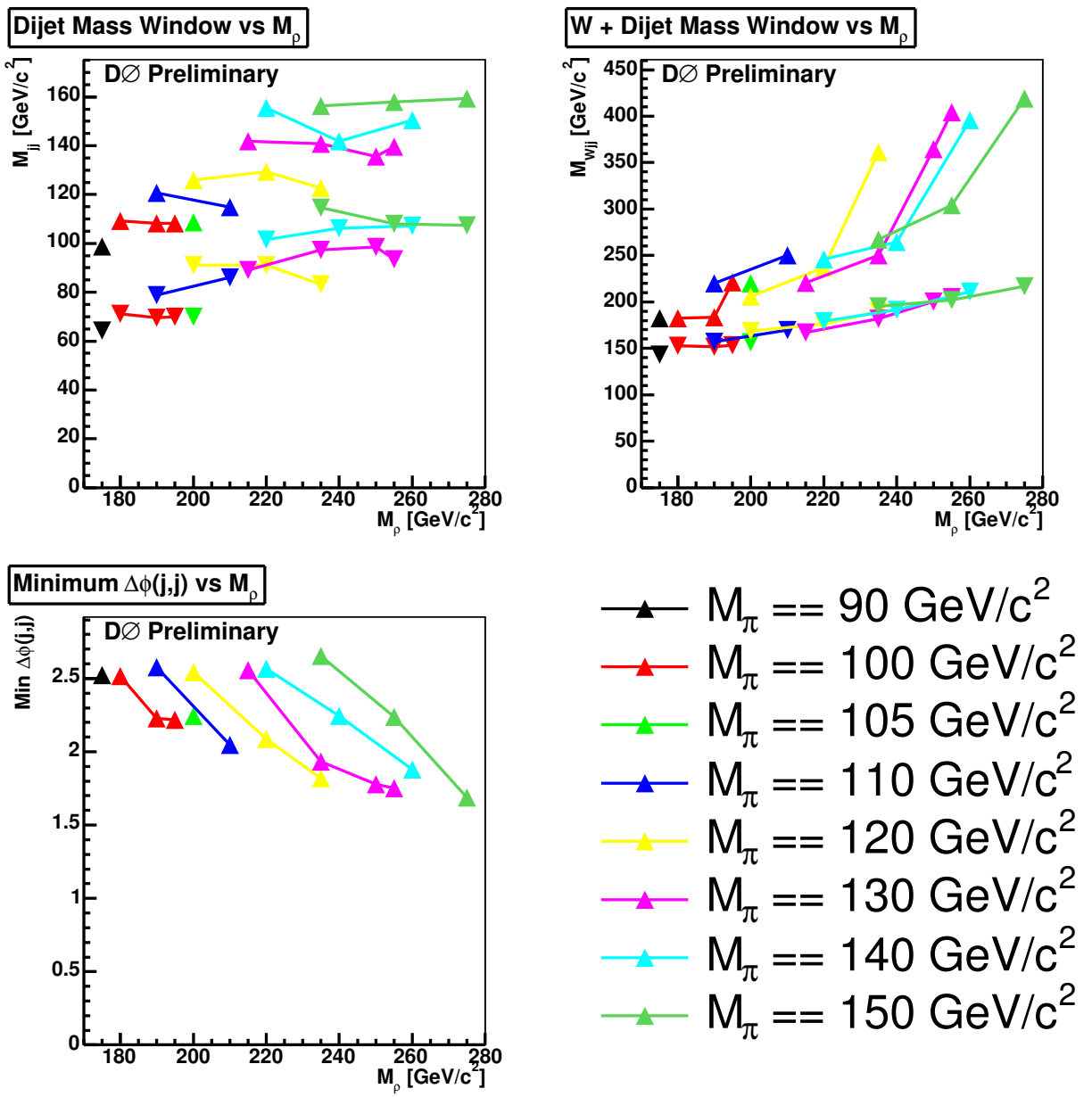

Figure 6.11: Optimized selection requirements on $m_{j j}$ (top left), $m_{W j j}$ (top right) and $\Delta \phi(j, j)$ (bottom left) as a function of the assumed $M_{\rho}$ and $M_{\pi}$. 
Dijet Mass Window

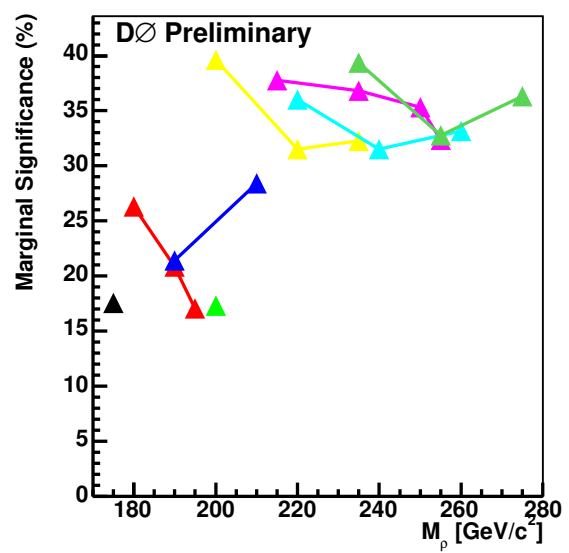

Minimum $\Delta \phi(\mathrm{j}, \mathrm{j})$

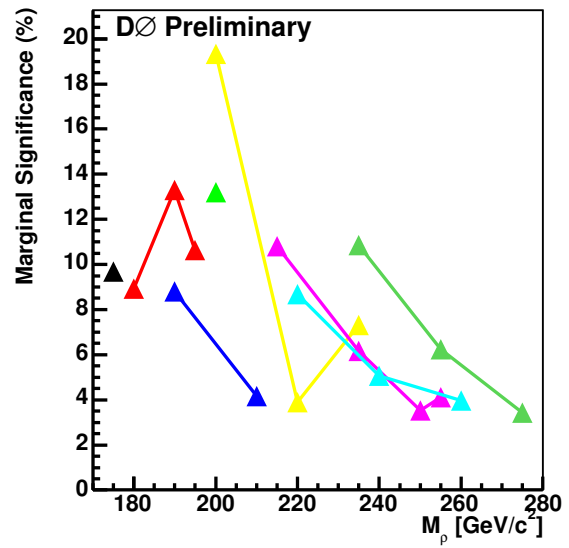

W + Dijet Mass Window

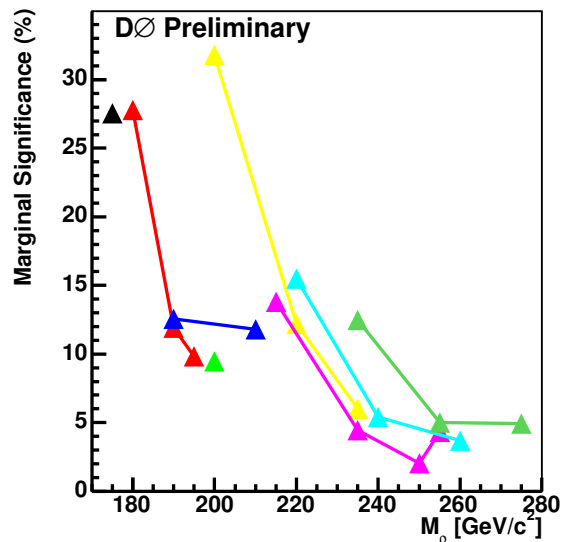

$\longrightarrow \mathrm{M}_{\pi}==90 \mathrm{GeV} / \mathrm{c}^{2}$

$\longrightarrow \mathrm{M}_{\pi}==100 \mathrm{GeV} / \mathrm{c}^{2}$

$\because \mathrm{M}_{\pi}==105 \mathrm{GeV} / \mathrm{c}^{2}$

$\rightarrow \mathrm{M}_{\pi}==110 \mathrm{GeV} / \mathrm{c}^{2}$ $\mathrm{M}_{\pi}==120 \mathrm{GeV} / \mathrm{c}^{2}$

$\because \mathrm{M}_{\pi}==130 \mathrm{GeV} / \mathrm{c}^{2}$

$\therefore \mathrm{M}_{\pi}==140 \mathrm{GeV} / \mathrm{c}^{2}$

$\rightarrow M_{\pi}==150 \mathrm{GeV} / \mathrm{c}^{2}$

Figure 6.12: Fractional change in signal significance when requirements on the dijet mass (top left), $W j j$ mass (top right) or dijet azimuthal opening angle (bootom left) are removed. 

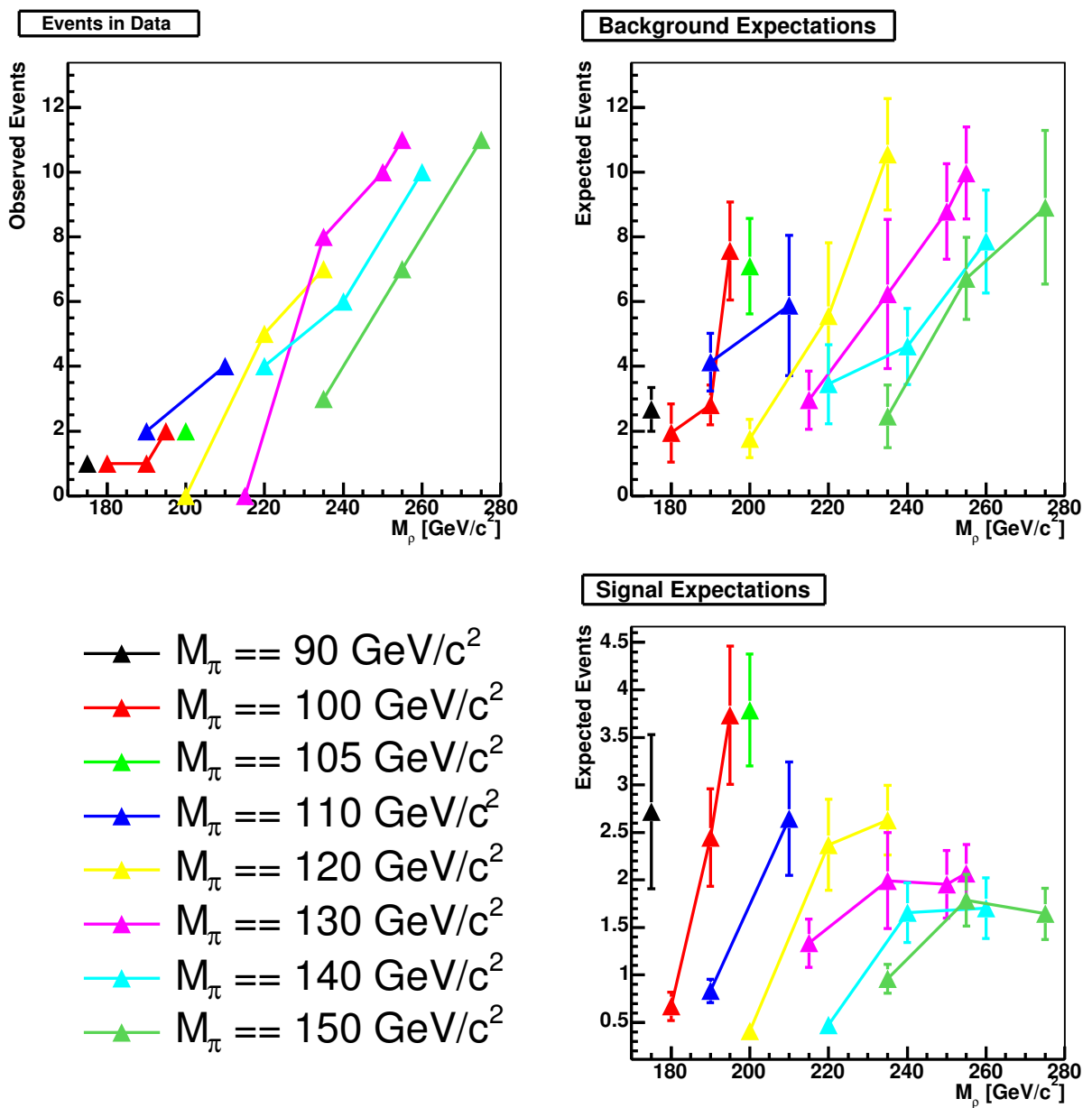

Figure 6.13: Final event yields from collider data (top left) the Standard Model (top right) and Technicolor (bottom right). The error bars indicate the full systematic uncertainties, but not the statistical widths of the predictions. 


\section{Chapter 7}

\section{Analysis and Results}

Before any definitive statement can be made regarding the validity of Technicolor, it is neccesssary to evaluate the systematic uncertainties on the Standard Model expectations as well as those on the signal predictions. These are summarized in Section 7.1. No significant excess over the expected background is observed. Therefore, a Bayesian technique, described in Section 7.2 is used to extract limits on the Technicolor production cross sections. The results are presented in Section 7.3.

\subsection{Systematic Uncertainties}

Table 7.1 and Appendix D summarize the systematic uncertainties on the signal and background predictions. The sources of uncertainty accounted for are:

The non- $W / Z$ background estimate: As discussed in Section 6.3, a $100 \%$ systematic uncertainty has been assigned to the prediction of the instrumental backgrounds.

Simulated cross sections: Uncertainties have been assigned to the background cross sections based on variations of the renormalization scale; the size of NNLO corrections; and uncertainty in the mass of the top quark (see Section 6.1.1).

Jet energy scale: The uncertainties in the jet energy calibration are propagated by varying the correction factors (shown in Figures 3.8 and 3.9) applied to the simulated data and rerunning the event selection. The correction factors are varied by the sum, in quadrature, of uncertainties for the collider data and the simulated data. 


\begin{tabular}{|l|lllllll|}
\hline sample & $W$ sel. & Dijet sel. & $b$-tag & $3^{\text {rd }}$ jet & $2^{\text {nd }}$ lep & $L_{T}$ & $p_{T}^{j j}$ \\
\hline \hline Non- $W / Z$ BG Estimate & 3900 & 280 & 15 & 11 & 9.9 & 6.8 & 5.8 \\
Cross sections & 9700 & 440 & 9.5 & 7.0 & 6.6 & 6.5 & 5.5 \\
MC statistics & 250 & 33 & 0.85 & 0.67 & 0.66 & 0.66 & 0.60 \\
Diboson kinematics & 1.7 & 0.85 & 0.030 & 0.024 & 0.021 & 0.020 & 0.016 \\
Jet energy scale & 0 & -464.96 & -10.49 & -6.3 & -6.4 & -6.4 & -5.0 \\
$b / c$-jet TRF's & 0 & 0 & 7.4 & 4.3 & 3.9 & 3.8 & 3.0 \\
Mistag TRF's & 0 & 0 & -2.1 & -1.5 & -1.4 & -1.4 & -1.2 \\
Jet smearing & 0 & 61 & 0.46 & -0.87 & -1.1 & -0.97 & -1.1 \\
Track smearing & 0 & -22.67 & -0.30 & -0.90 & -0.93 & -1.4 & -0.89 \\
PV scale factor & 0 & 120 & 6.2 & 3.9 & 3.5 & 3.5 & 2.8 \\
Jet scale factor & 0 & -244.71 & -6.6 & -4.0 & -3.7 & -3.8 & -5.0 \\
Taggability scale factor & 0 & 7.9 & -6.8 & -1.6 & -1.4 & -1.7 & -2.8 \\
\hline Total BG prediction & 180000 & 3300 & 140 & 88 & 80 & 76 & 62 \\
Total BG error & 10000 & 750 & 25 & 16 & 15 & 13 & 12 \\
\hline
\end{tabular}

Table 7.1: Evolution of the systematic uncertainties on the backround with initial selection requirements.

Tagging efficiencies: The impact of uncertainties in the $b$-tagging TRFs is measured by varying these efficiencies by their uncertainties, as described in Section 5.4, and repeating the selection process. The $b$ - and $c$-jet TRFs were assumed to be correlated, and varied together. The mistag rate was varied independently.

Detector Modeling: Corrections for the detector modeling include the efficiency scale factors and momentum smearing discussed in Chapter 4, as well as the taggability efficiency from Section 5.1. Uncertainties for each of these effects were estimated by repetition of the event selection with the correction removed.

In addition there is a 5\% uncertainty assigned to the effective luminosity, based on that of the $W$ cross section [59]. Note that the treatment of the non- $W / Z$ backgrounds and the detector modeling effects is quite conservative. A more realistic treatment would involve variation of the parameterizations with their uncertainties and is an avenue for future improvement of this analysis. In the case of the instrumental backgrounds, this would require a better understanding of the estimation technique and the control samples.

The limit setting method takes as an input only the signal acceptance, not the Technicolor cross sections. While the cross sections are used in determining 
the weighted average of the acceptances for the different charge states, the associated uncertainties are correlated, and therefore cancel. Thus, it is not necessary evaluate the impact of uncertainties in the signal cross sections.

\subsection{Limit Setting Technique}

A Bayesian model is used to obtain upper limits on Technicolor production cross sections [64], [65], [66]. In this approach a probability density $p(\sigma \mid n, I)$ for the signal cross section, $\sigma$, is constructed, given that $n$ events were observed in the collider data. Measurements of the luminosity, signal efficiency times acceptance and expected background are indicated by $I(\mathcal{L}, \epsilon$ and $B$, respectively).

An upper limit, $\sigma_{U L}$, on the cross section is obtained with some confidence level $\beta$ (in this case $\beta=95 \%$ ) when: ${ }^{1}$

$$
\beta=\int_{0}^{\sigma_{U L}} p(\sigma \mid n, I) d \sigma
$$

The task, then, is to construct this posterior probability density $p(\sigma \mid n, I)$. According to Bayes' theorem this may be obtained from the model $(M(n \mid \sigma, \mathcal{L}, \epsilon, B))$ and prior $(p(\sigma, \mathcal{L}, \epsilon, B \mid I))$ distributions:

$$
p(\sigma, \mathcal{L}, \epsilon, B \mid n, I)=\frac{M(n \mid \sigma, \mathcal{L}, \epsilon, B) p(\sigma, \mathcal{L}, \epsilon, B \mid I)}{\int d \sigma \int d \mathcal{L} \int d \epsilon \int d B M(n \mid \sigma, \mathcal{L}, \epsilon, B) p(\sigma, \mathcal{L}, \epsilon, B \mid I)}
$$

The model distribution is the likelihood to observe $n$ events in the data if the theory being considered is correct. It is, therefore, a Poisson with mean $B+\mathcal{L} \epsilon \sigma:$

$$
M(n \mid \sigma, \mathcal{L}, \epsilon, B, I)=\frac{(B+\mathcal{L} \epsilon \sigma)^{n} \exp (B+\mathcal{L} \epsilon \sigma)}{n !}
$$

The prior distribution describes the direct knowledge of $\sigma, \mathcal{L}, \epsilon$ and $B$. Since $I$ constrains the signal cross section only through equation 7.2 , this factorizes as $p(\sigma) p(\mathcal{L}, \epsilon, B \mid I)$. Lacking any direct information regarding $\sigma, p(\sigma)$ (thus referred to as an uninformative prior) is taken to have flat over the interval $\left[0, \sigma_{\max }\right]$ and zero elsewhere (with $\sigma_{\max }$ chosen to be sufficiently large that this is a good assumption).

\footnotetext{
${ }^{1}$ Provided, of course, that the data are consistent with the expected background.
} 


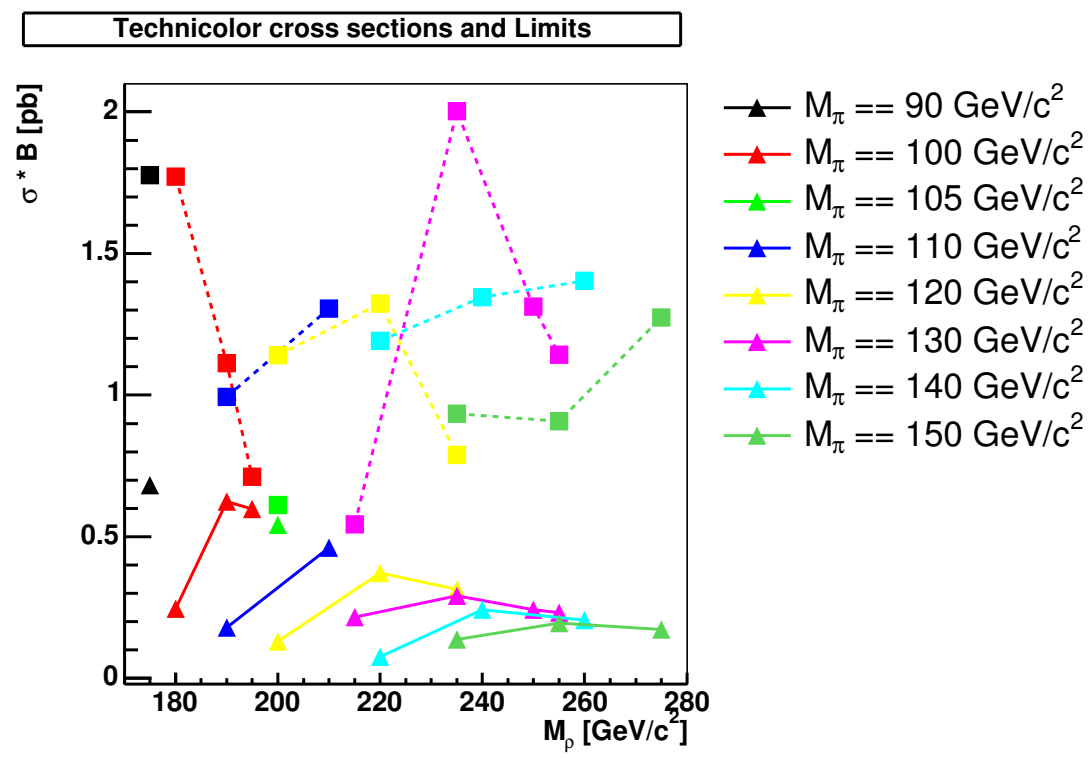

Figure 7.1: Production cross sections (triangles with solid lines) and limits (squares with dashed lines) for $\rho_{T} \rightarrow W \pi_{T} \rightarrow \mu \nu b \bar{b} / b \bar{c}$ with $M_{V}=100 \mathrm{GeV}$.

Because of the direct measurements, with uncertainties of the remaining parameters, $p(\sigma) p(\mathcal{L}, \epsilon, B \mid I)$ may be expressed as a multivariate gaussian. Equation 7.2 may then be integrated over $\mathcal{L}, \epsilon$ and $B$ to obtain $p(\sigma \mid n)$. This, in turn, allows evaluation of the integral from equation 7.1 , which may be solved to obtain a $95 \%$ confidence level upper limit on the signal cross section.

\subsection{Limits on Technicolor Production}

The event yields are consistent with Standard Model expectations. When both the systematic uncertainties and the Poisson widths of the predictions are included, at most a $0.65 \sigma$ excess (for $M_{\rho}=140 \mathrm{GeV} / c^{2}, M_{\pi}=260 \mathrm{GeV} / c^{2}$ ) or a $1.7 \sigma$ deficit (for $M_{\rho}=195 \mathrm{GeV} / c^{2}, M_{\pi}=100 \mathrm{GeV} / c^{2}$ ). Therefore, 95\% confidence level upper limits are set on the Technicolor production cross sections. Table 7.2 summarizes the results for each mass hypothesis, assuming a value of $100 \mathrm{GeV}$ for the scale parameter, $M_{V}$. The limits and production cross sections are plotted in Figure 7.1. None of the considered mass points are ruled out for this choice of $M_{V}$.

However, it is possible to reinterpret the results for the choice $M_{V}=500 \mathrm{GeV}$. While the overall cross sections do depend on $M_{V}$, the kinematic distributions 


\begin{tabular}{|ll|lll|ll|}
\hline$M_{\pi}\left(\mathrm{GeV} / c^{2}\right)$ & $M_{\rho}\left(\mathrm{GeV} / c^{2}\right)$ & $N_{\text {obs }}$ & Background & Acceptance & $\sigma \times B(p b)$ & limit $(p b)$ \\
\hline 90 & 175 & 1 & $2.7 \pm 0.68$ & $2.0 \pm 0.59$ & 0.68 & 1.8 \\
100 & 180 & 1 & $1.9 \pm 0.90$ & $1.4 \pm 0.30$ & 0.25 & 1.8 \\
100 & 190 & 1 & $2.8 \pm 0.62$ & $2.0 \pm 0.41$ & 0.62 & 1.1 \\
110 & 190 & 2 & $4.1 \pm 0.89$ & $2.3 \pm 0.33$ & 0.18 & 0.99 \\
100 & 195 & 2 & $7.6 \pm 1.5$ & $3.1 \pm 0.61$ & 0.60 & 0.71 \\
105 & 200 & 2 & $7.1 \pm 1.5$ & $3.5 \pm 0.54$ & 0.54 & 0.61 \\
120 & 200 & 0 & $1.8 \pm 0.60$ & $1.6 \pm 0.36$ & 0.13 & 1.1 \\
110 & 210 & 4 & $5.9 \pm 2.2$ & $2.9 \pm 0.64$ & 0.46 & 1.3 \\
130 & 215 & 0 & $3.0 \pm 0.90$ & $3.1 \pm 0.59$ & 0.22 & 0.54 \\
120 & 220 & 5 & $5.6 \pm 2.3$ & $3.2 \pm 0.64$ & 0.37 & 1.3 \\
140 & 220 & 4 & $3.5 \pm 1.2$ & $3.1 \pm 0.54$ & 0.076 & 1.2 \\
120 & 235 & 7 & $11 . \pm 1.7$ & $4.2 \pm 0.58$ & 0.31 & 0.79 \\
130 & 235 & 8 & $6.2 \pm 2.3$ & $3.4 \pm 0.86$ & 0.29 & 2.0 \\
150 & 235 & 3 & $2.5 \pm 0.97$ & $3.5 \pm 0.55$ & 0.14 & 0.93 \\
140 & 240 & 6 & $4.6 \pm 1.2$ & $3.4 \pm 0.64$ & 0.24 & 1.3 \\
130 & 250 & 10 & $8.8 \pm 1.5$ & $4.0 \pm 0.73$ & 0.24 & 1.3 \\
130 & 255 & 11 & $10 . \pm 1.4$ & $4.4 \pm 0.65$ & 0.23 & 1.1 \\
150 & 255 & 7 & $6.7 \pm 1.3$ & $4.6 \pm 0.69$ & 0.19 & 0.91 \\
140 & 260 & 10 & $7.9 \pm 1.6$ & $4.1 \pm 0.77$ & 0.21 & 1.4 \\
150 & 275 & 11 & $8.9 \pm 2.4$ & $4.8 \pm 0.78$ & 0.17 & 1.3 \\
\hline
\end{tabular}

Table 7.2: Results of the analysis for various technicolor masses for $M_{V}=100 \mathrm{GeV}$. Shown for each mass point are the number of events observed in data, the background expectation, the signal acceptance, the total signal cross section times branching fraction and the $95 \%$ confidence limit on the cross section. 


\begin{tabular}{|ll|lll|ll|}
\hline$M_{\pi}\left(\mathrm{GeV} / c^{2}\right)$ & $M_{\rho}\left(\mathrm{GeV} / c^{2}\right)$ & $N_{o b s}$ & Background & Acceptance & $\sigma \times B(p b)$ & limit $(p b)$ \\
\hline 90 & 175 & 1 & $2.7 \pm 0.68$ & $2.0 \pm 0.60 \%$ & 1.1 & 1.8 \\
100 & 180 & 1 & $1.9 \pm 0.90$ & $1.3 \pm 0.31 \%$ & 0.37 & 1.8 \\
100 & 190 & 1 & $2.8 \pm 0.62$ & $2.0 \pm 0.41 \%$ & 0.94 & 1.1 \\
110 & 190 & 2 & $4.1 \pm 0.89$ & $2.3 \pm 0.35 \%$ & 0.24 & 0.98 \\
100 & 195 & 2 & $7.6 \pm 1.5$ & $3.1 \pm 0.61 \%$ & 0.95 & 0.72 \\
105 & 200 & 2 & $7.1 \pm 1.5$ & $3.5 \pm 0.54 \%$ & 0.84 & 0.61 \\
120 & 200 & 0 & $1.8 \pm 0.60$ & $1.5 \pm 0.39 \%$ & 0.15 & 1.3 \\
110 & 210 & 4 & $5.9 \pm 2.2$ & $2.9 \pm 0.65 \%$ & 0.70 & 1.3 \\
130 & 215 & 0 & $3.0 \pm 0.90$ & $3.0 \pm 0.57 \%$ & 0.26 & 0.55 \\
120 & 220 & 5 & $5.6 \pm 2.3$ & $3.1 \pm 0.63 \%$ & 0.54 & 1.3 \\
140 & 220 & 4 & $3.5 \pm 1.2$ & $3.0 \pm 0.57 \%$ & 0.070 & 1.3 \\
120 & 235 & 7 & $11 . \pm 1.7$ & $4.1 \pm 0.57 \%$ & 0.47 & 0.79 \\
130 & 235 & 8 & $6.2 \pm 2.3$ & $3.4 \pm 0.86 \%$ & 0.43 & 2.0 \\
150 & 235 & 3 & $2.5 \pm 0.97$ & $3.5 \pm 0.57 \%$ & 0.14 & 0.96 \\
140 & 240 & 6 & $4.6 \pm 1.2$ & $3.4 \pm 0.65 \%$ & 0.35 & 1.3 \\
130 & 250 & 10 & $8.8 \pm 1.5$ & $4.0 \pm 0.71 \%$ & 0.37 & 1.3 \\
130 & 255 & 11 & $10 . \pm 1.4$ & $4.4 \pm 0.65 \%$ & 0.35 & 1.1 \\
150 & 255 & 7 & $6.7 \pm 1.3$ & $4.5 \pm 0.67 \%$ & 0.29 & 0.92 \\
140 & 260 & 10 & $7.9 \pm 1.6$ & $4.1 \pm 0.77 \%$ & 0.32 & 1.4 \\
150 & 275 & 11 & $8.9 \pm 2.4$ & $4.7 \pm 0.76 \%$ & 0.25 & 1.3 \\
\hline
\end{tabular}

Table 7.3: Results of the analysis for various technicolor masses for $M_{V}=500 \mathrm{GeV}$. Shown for each mass point are the number of events observed in data, the background expectation, the signal acceptance, the total signal cross section times branching fraction and the $95 \%$ confidence limit on the cross section.

are unchanged. The relative cross sections for the different charge states also depend on $M_{V}$ so the weighted average of the signal acceptance will also vary. Therefore, it is only necessary to apply the limit calculator with the new cross sections and reweighted efficiencies. The results for $M_{V}=500 \mathrm{GeV}$ are summarized in Table 7.3 and Figure 7.2. In this case it is possible to exclude the mass points $M_{\rho}=195 \mathrm{GeV} / c^{2}, M_{\pi}=100 \mathrm{GeV} / c^{2}$ and $M_{\rho}=200 \mathrm{GeV} / c^{2}$, $M_{\pi}=105 \mathrm{GeV} / c^{2}$. 


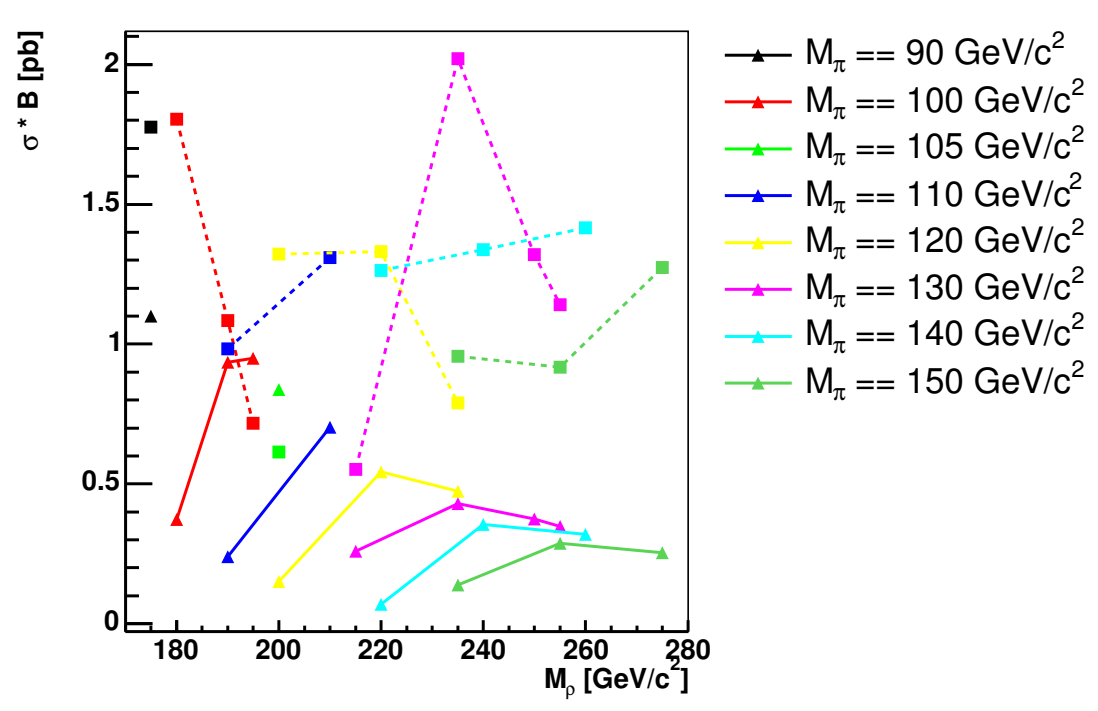

Figure 7.2: Production cross sections (triangles with solid lines) and limits (squares with dashed lines) for $\rho_{T} \rightarrow W \pi_{T} \rightarrow \mu \nu b \bar{b} / b \bar{c}$ with $M_{V}=500 \mathrm{GeV}$. 


\section{Chapter 8}

\section{Conclusions and Outlook}

A search for the production of technirho mesons, in the decay channel $\rho_{T} \rightarrow W \pi_{T} \rightarrow \mu \nu b \bar{b} / b \bar{c}$, has been conducted at $\mathrm{D} \varnothing$. The data are consistent with the expectations for Standard Model backgrounds. Therefore upper limits on the cross sections for twenty hypothetical combinations of the $\rho_{T}$ and $\pi_{T}$ masses have been set.

These limits may be interpreted to rule out, at the $95 \%$ confidence level, the mass combinations $M_{\rho}=195 \mathrm{GeV} / c^{2}, M_{\pi}=100 \mathrm{GeV} / c^{2}$ and $M_{\rho}=200 \mathrm{GeV} / c^{2}$, $M_{\pi}=105 \mathrm{GeV} / c^{2}$, when the scale parameter $M_{V}$ is assumed to be $500 \mathrm{GeV}$. No mass hypotheses are ruled our, given $M_{V}=100 \mathrm{GeV}$.

It should be noted, however, that the future prospects for this search are good. The limits are generally within an order of magnitude of the expected cross sections, and in some cases differ by less than a factor of two. Improved analysis techniques, combined with much larger data sets promise definitive answers regarding the validity of Technicolor.

\subsection{Other Recent Technicolor Searches}

In parallel to the analysis presented herein, a similar search has been conducted in the $W \pi_{T} \rightarrow e \nu b \bar{b} / b \bar{c}$ channel [18], [43]. The electron plus jets result used a $388 \mathrm{pb}^{-1}$ data set, also recorded by $\mathrm{D} \emptyset$. A simple cut-based analysis was used.

The grid of assumed masses was finer, and extended to a lower values than the set used for this analysis. Nevertheless, the two results have several points in common, and they are compared, for $M_{V}=100 \mathrm{GeV}$, in Table 8.1. With this choice of $M_{V}$, technipion masses from $95 \mathrm{GeV} / c^{2}$ to $96 \mathrm{GeV} / c^{2}$ were ruled out, assuming a technirho mass of $190 \mathrm{GeV} / c^{2}$. Also excluded is the region $M_{\rho}=200 \mathrm{GeV} / c^{2}, M_{\pi}=100 \mathrm{GeV} / c^{2}-105 \mathrm{GeV} / c^{2}$. 


\begin{tabular}{|cc|cc|}
\hline$M_{\pi}\left(\mathrm{GeV} / c^{2}\right)$ & $M_{\rho}\left(\mathrm{GeV} / c^{2}\right)$ & $\mu(\mathrm{pb})$ & $\mathrm{e}(\mathrm{pb})$ \\
\hline 90 & 175 & 1.8 & 1.4 \\
100 & 180 & 1.8 & 0.99 \\
100 & 190 & 1.1 & 0.86 \\
100 & 195 & 0.71 & 0.64 \\
105 & 200 & 0.61 & 0.6 \\
110 & 210 & 1.3 & 0.73 \\
130 & 215 & 0.54 & 0.66 \\
120 & 220 & 1.3 & 0.93 \\
\hline
\end{tabular}

Table 8.1: Comparison of the cross section limits between the electron and muon channels for $M_{V}=100 \mathrm{GeV}$ [18].

\begin{tabular}{cc}
\hline$M_{\rho}\left(\mathrm{GeV} / c^{2}\right)$ & $M_{\pi}\left(\mathrm{GeV} / c^{2}\right)$ Excluded at $95 \% \mathrm{CL}$ \\
\hline 180 & $89.7-90.4$ \\
185 & $92.6-97.1$ \\
190 & $91.8-102.2$ \\
195 & $96.7-107.4$ \\
200 & $96.8-111.6$ \\
205 & $99.0-114.9$ \\
210 & $101.9-115$ \\
215 & $107.2-115$ \\
\hline
\end{tabular}

Table 8.2: Exclusion region at $M_{V}=500 \mathrm{GeV}$ for the electron plus jets Technicolor search [18].

In the case of $M_{V}=500 \mathrm{GeV}$, the search in the electron channel is able to exclude a region of the $M_{\rho}-M_{\pi}$ plane (see Table 8.2). The greater sensitivity of the electron channel is primarily a result of the larger data set and a higher trigger trigger efficiency.

At the time of this writing, an improved version of the electron search has just been completed [67]. The data set analyzed is the same as the analysis just discussed. However, the event selection was the minimum required to identify the e $\nu b j$ sample. Topological variables (but not the dijet and $W$ plus dijet masses) were fed to three separate neural networks.

Each of these networks was trained to reject a certain class of backgrounds: continuum $W$ plus light and charm jet production; $W b \bar{b}$ production; and events containing a top quark. The outputs of these three networks was then fed to 
a fourth network. The output of this fourth network was used to define an event weight, for the purposes of filling a joint $m_{j j}-m_{W j j}$ distribution.

The Standard Model, signal and collider data distributions were compared, using a binned, maximum likelihood method, and 95\% CL upper limits were extracted. As shown in Figure 8.1, this approach expands the excluded region, but still allows some masses that the original analysis rules out.

\subsection{Prospects for Improvement}

In the near future, there is great promise for establishing the validity of Technicolor, or of refuting it. This dissertation has presented a test of this model using a $291 \mathrm{pb}^{-1}$ data set, while the corresponding analysis in the electron channel makes use of $388 \mathrm{pb}^{-1}$. However, with the recent completion of the Tevatron Run IIa, the D $\varnothing$ detector has recorded an integrated luminosity of $\sim 1.2 \mathrm{fb}^{-1}$. The CDF experiment has collected a sample of comparable size.

Therefore, simply extending these analyses to already existing data sets offers a relatively straightforward improvement in sensitivity. The current experimental reach may be further enhanced by combination of the muon and electron results. The CDF collaboration does not presently have a Technicolor search (notwithstanding their Run I result, which uses an old model). However, if they produce one, a combined Tevatron result should be pursued.

As Run IIb commences, a total luminosity of $4-8 \mathrm{fb}^{-1}$ per experiment is expected. This will be recorded with improved trigger and tracking systems that promise greater efficiencies (or better rejections).

Furthemore, the search techniques can also be improved. Only single muon triggers were used, but $\mathrm{D} \varnothing$ has recorded data with muon plus jet triggers as well. These gain rejection through the requirement of a jet, and may thus relax the muon trigger requirements, and extend the geometrical acceptance.

The electron channel has shown substantial improvement through the use of multivariate analysis techniques (neural networks and the comparison of distribution shapes). This approach should provide a similar benefit to the muon channel.

In addition to the JLIP tagging algorithm, heavy flavor jets may be identified through the reconstruction of a secondary vertices or muons that arise from the decay of hadrons that contain a $b$ - or $c$-quark. D $\varnothing$ is currently exploring the possibility of using a neural network to combine the results of all three approaches to produce a single tagger with a better performance.

The latest versions of the Standard Model $W H \rightarrow l \nu b \bar{b}$ search at D $\varnothing$ have extended their reach by creating orthogonal samples with a single, tight $b$-tag 


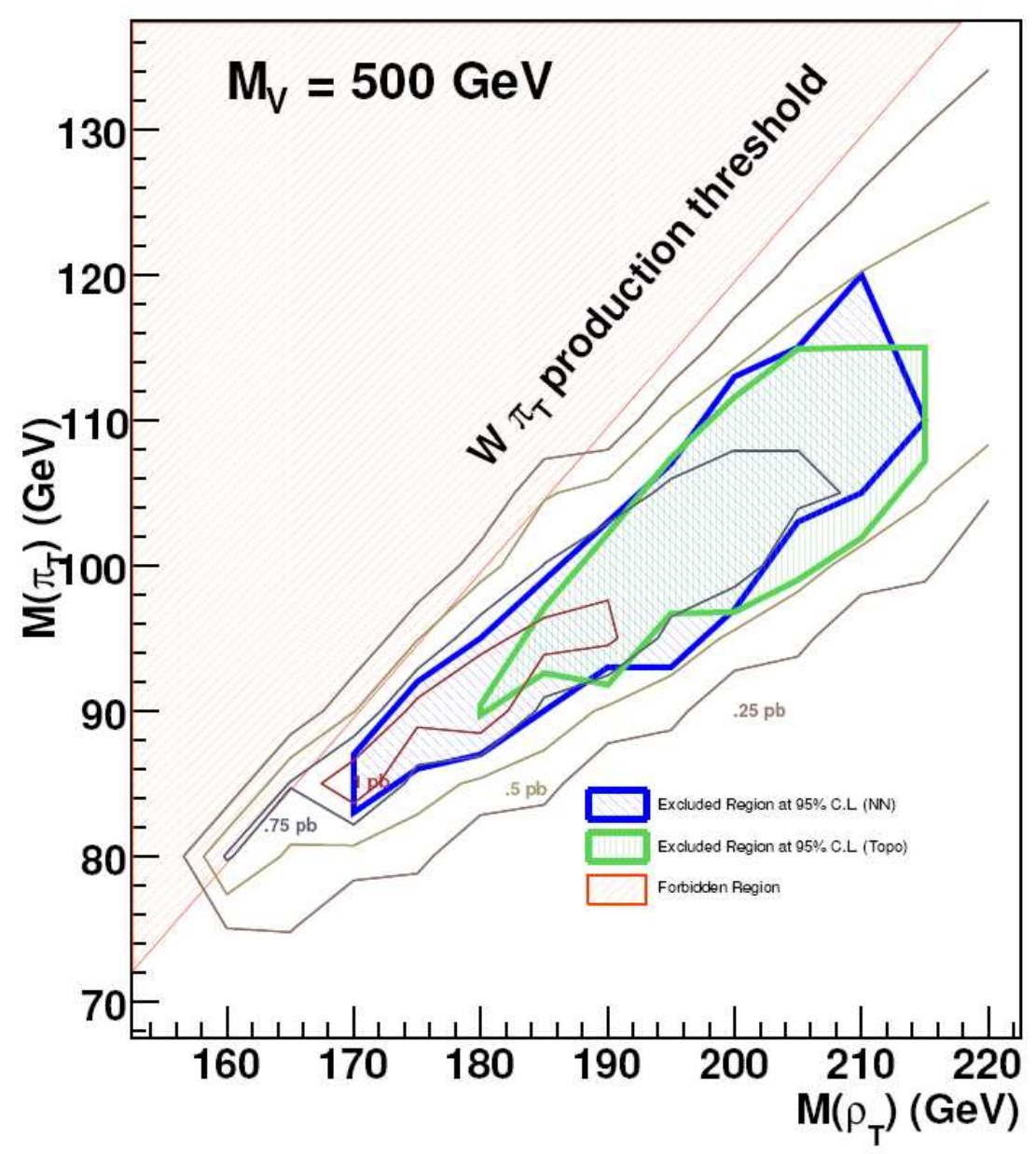

Figure 8.1: Excluded region for the $W \pi_{T} \rightarrow e \nu b j$ search using neural nets. The blue curve outlines the 95\% CL excluded region, while the green curve shows the corresponding region for the cut-based analysis on the same data $\operatorname{set}[67]$. 
or two loose $b$-tags [68]. This approach should benefit $W \pi_{T}$ searches as well.

Finally, it has been noted that this analysis takes a conservative approach in evaluating several systematic uncertainties. In the present, low statistics, sample, the improvement offered by a more realistic treatment is relatively small. However, as the data set size increases, the effect of the systematics will become more important.

\subsection{Further Ahead}

The Large Hadron Collider is expected to begin operations in roughly one year. While it will certainly take some time to commission and calibrate the detectors, the experimental program holds great promise. If the Tevatron does not provide a definitive test of Technicolor, the LHC certainly will. In either case, new insights into the nature of electroweak symmetry breaking may be expected soon. 


\section{Appendix A}

\section{Optimization Refinement Figures}

Presented here are figures showing the variation of the projected significance, $S / \sqrt{B+(0.3 B)^{2}}$ with cut value in the neighborhood of the initial RGS maxmimum, indicated by the vertical red lines. The location of the optimal selection requirements are shown with the blue lines. 

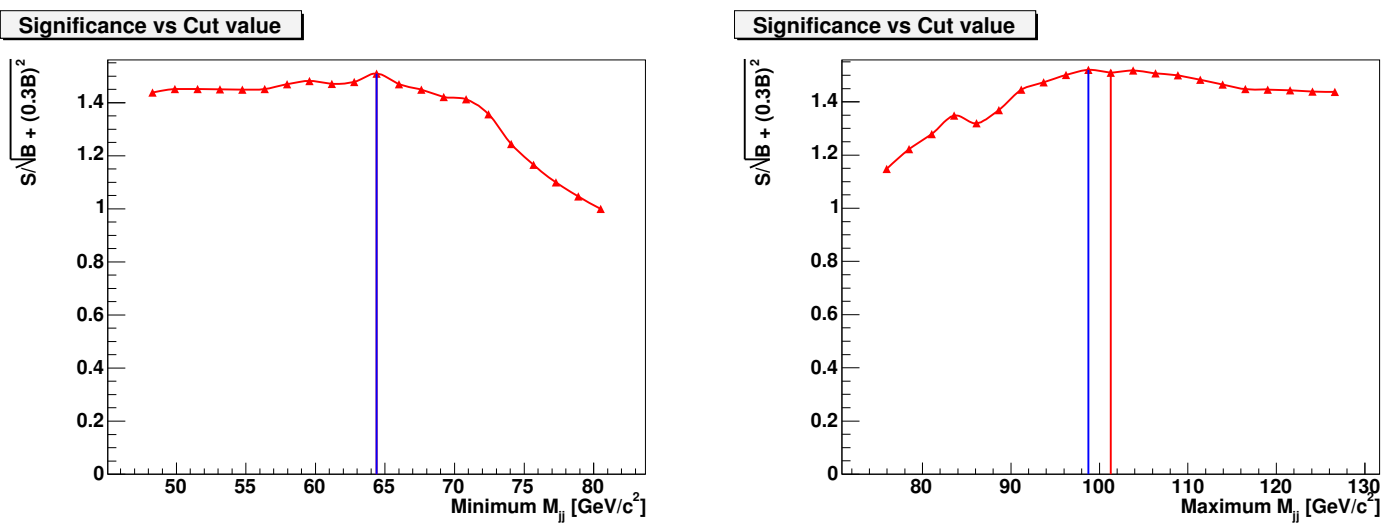

Significance vs Cut value

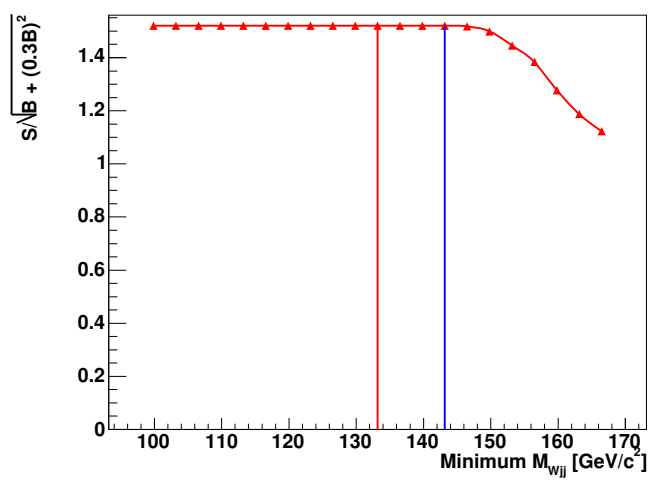

Significance vs Cut value

Significance vs Cut value
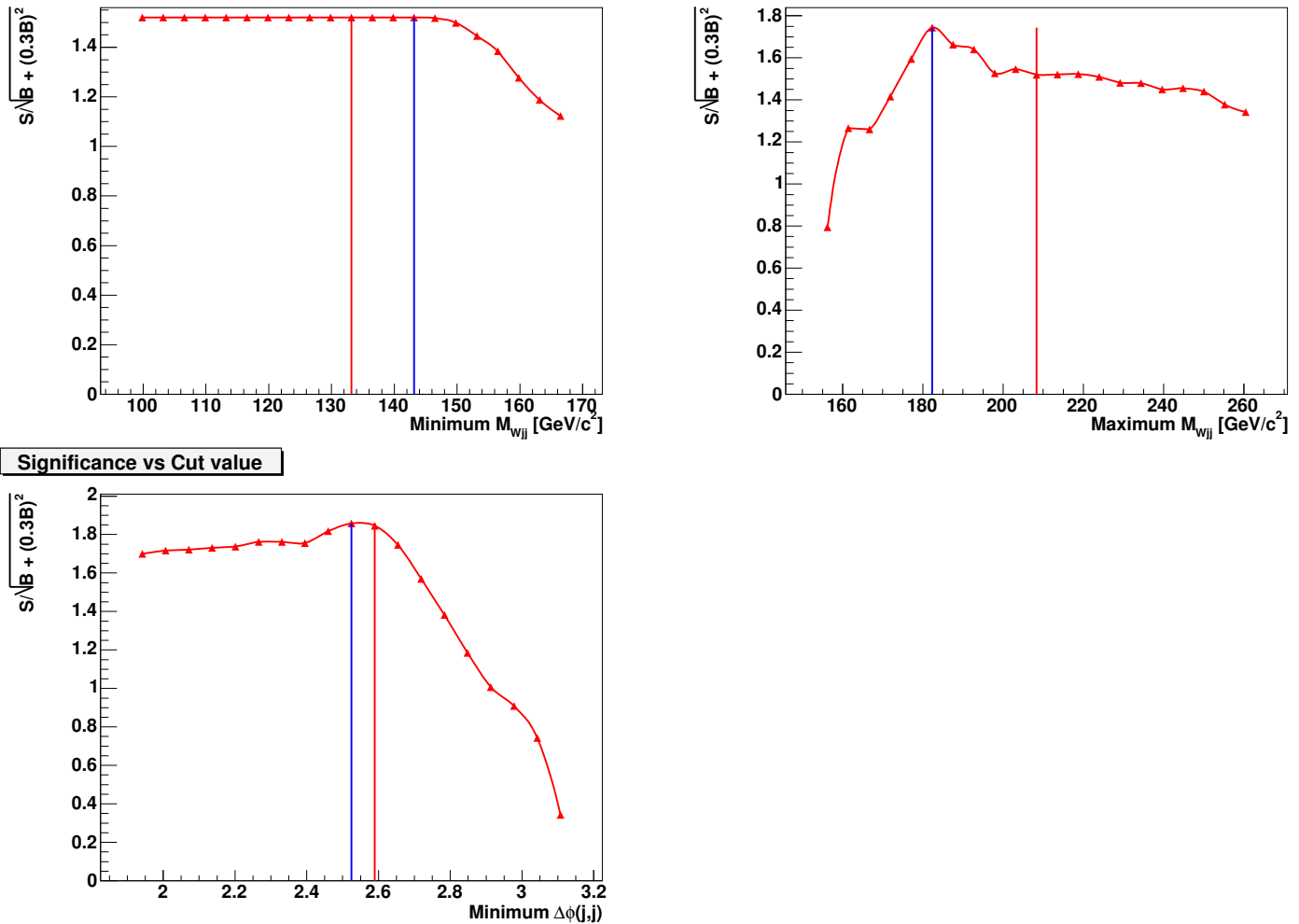

Figure A.1: Results of varying the selection cuts for $M_{\rho}=175 \mathrm{GeV} / c^{2}, M_{\pi}=$ $90 \mathrm{GeV} / c^{2}$. The vertical red line indicates the postion of the initial grid search maximum. The blue line indicates the new maximum after examining the area around the initial maximum. 


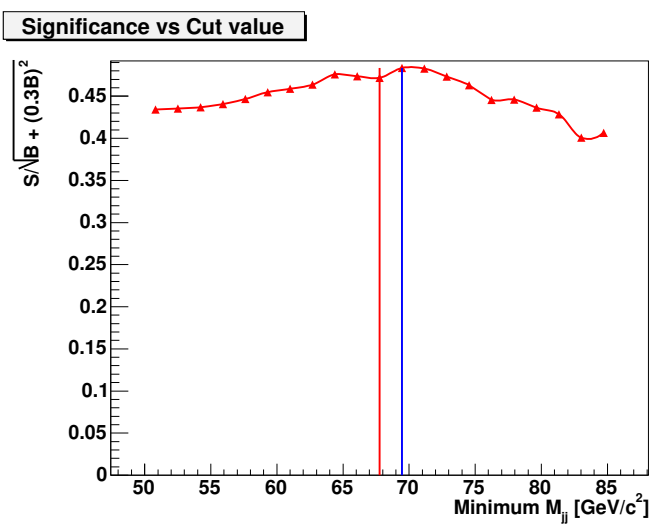

\section{Significance vs Cut value}

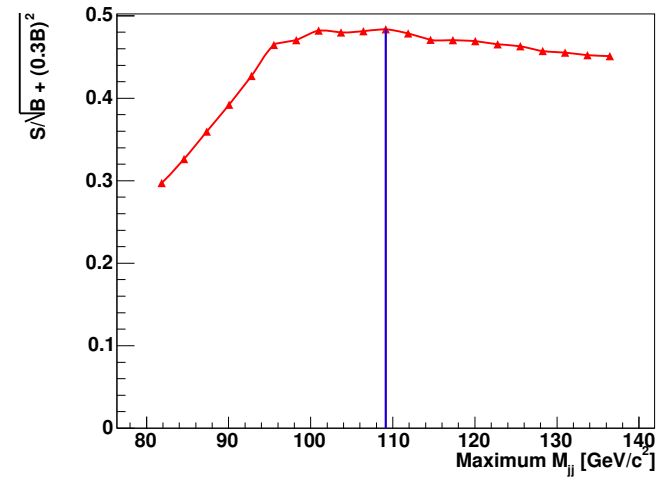

Significance vs Cut value

Significance vs Cut value

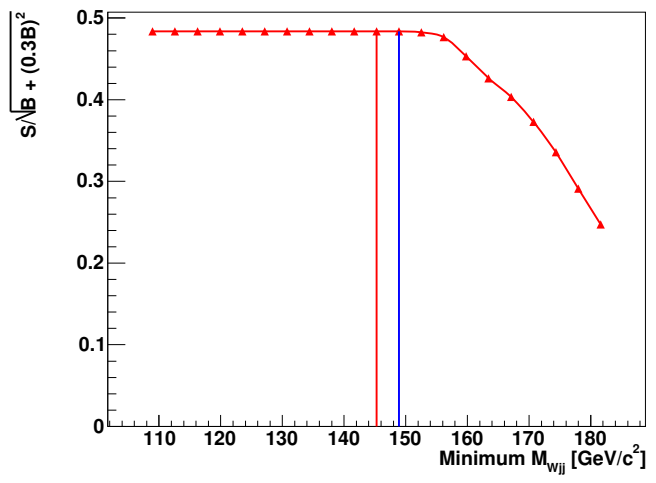

Significance vs Cut value
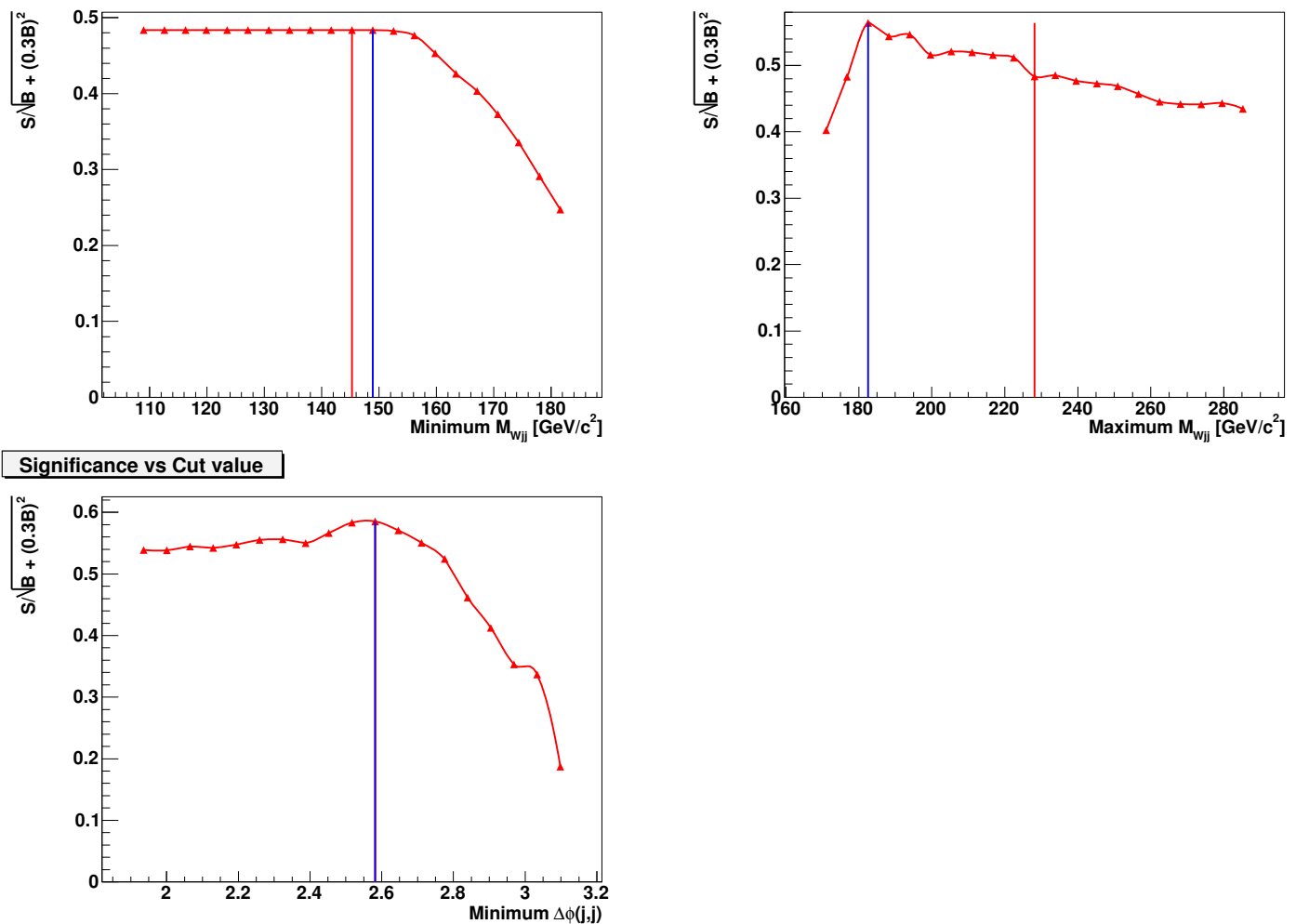

Figure A.2: Results of varying the selection cuts for $M_{\rho}=180 \mathrm{GeV} / c^{2}, M_{\pi}=$ $100 \mathrm{GeV} / c^{2}$. The vertical red line indicates the postion of the initial grid search maximum. The blue line indicates the new maximum after examining the area around the initial maximum. 

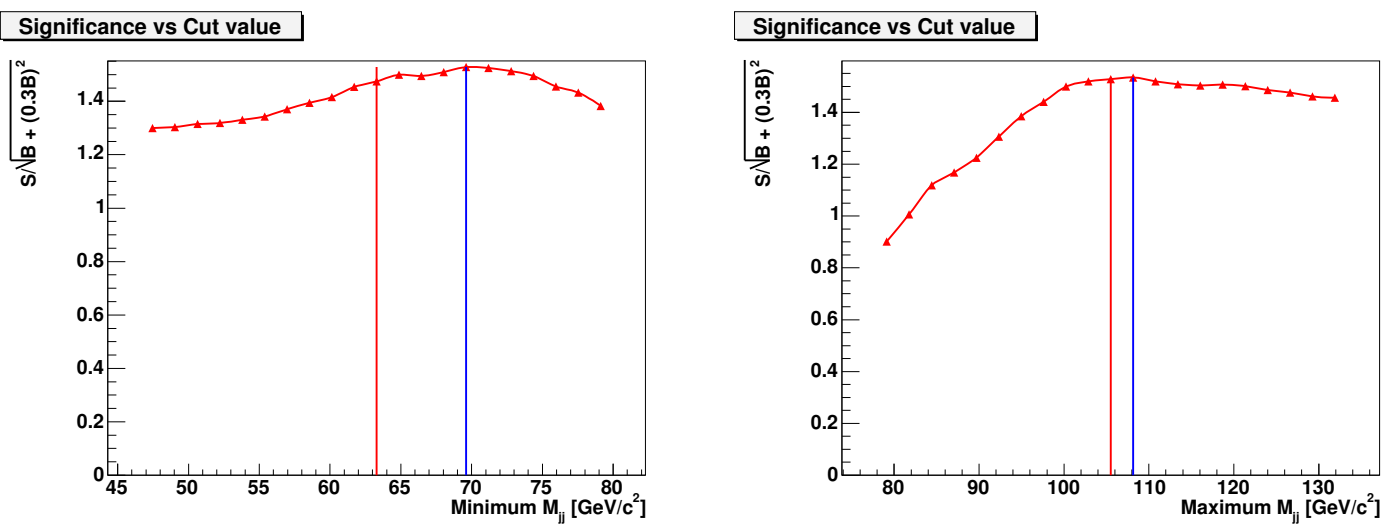

Significance vs Cut value

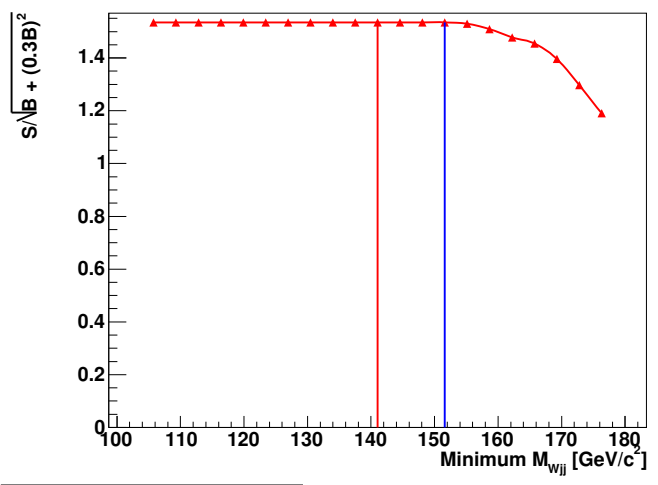

Significance vs Cut value

Significance vs Cut value
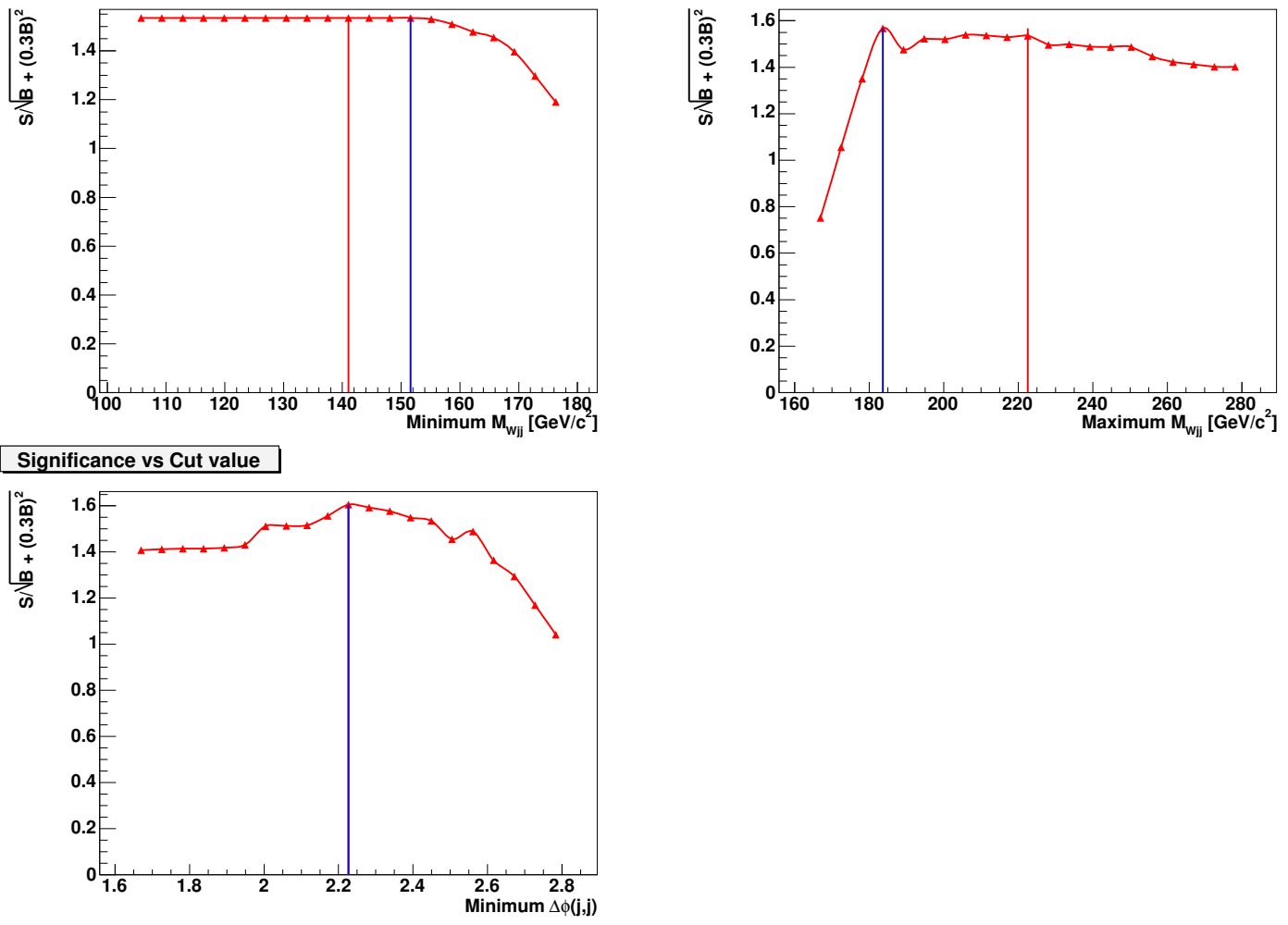

Figure A.3: Results of varying the selection cuts for $M_{\rho}=190 \mathrm{GeV} / c^{2}, M_{\pi}=$ $100 \mathrm{GeV} / c^{2}$. The vertical red line indicates the postion of the initial grid search maximum. The blue line indicates the new maximum after examining the area around the initial maximum. 

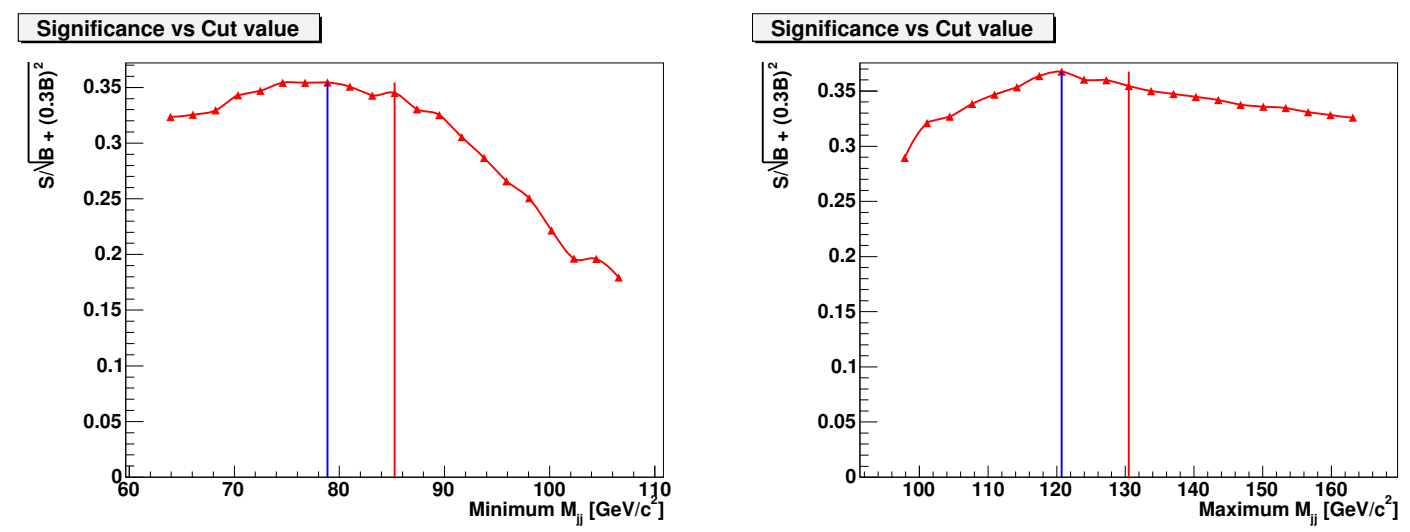

Significance vs Cut value

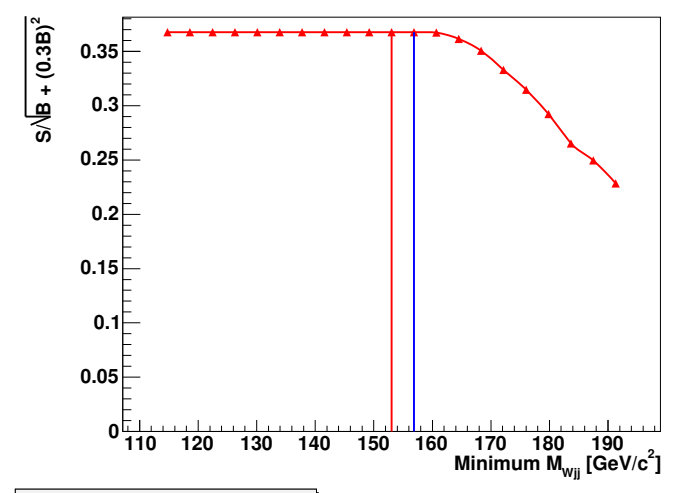

Significance vs Cut value

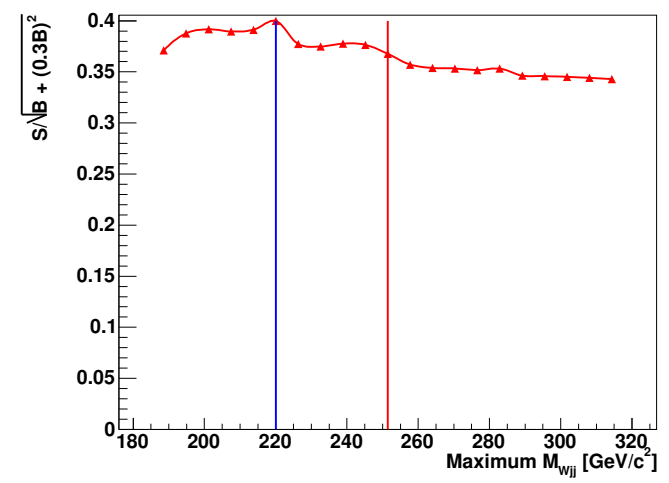

Significance vs Cut value

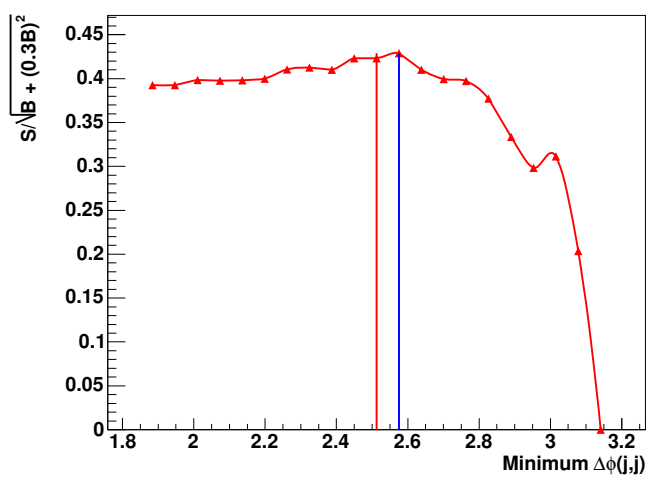

Figure A.4: Results of varying the selection cuts for $M_{\rho}=190 \mathrm{GeV} / c^{2}, M_{\pi}=$ $110 \mathrm{GeV} / c^{2}$. The vertical red line indicates the postion of the initial grid search maximum. The blue line indicates the new maximum after examining the area around the initial maximum. 

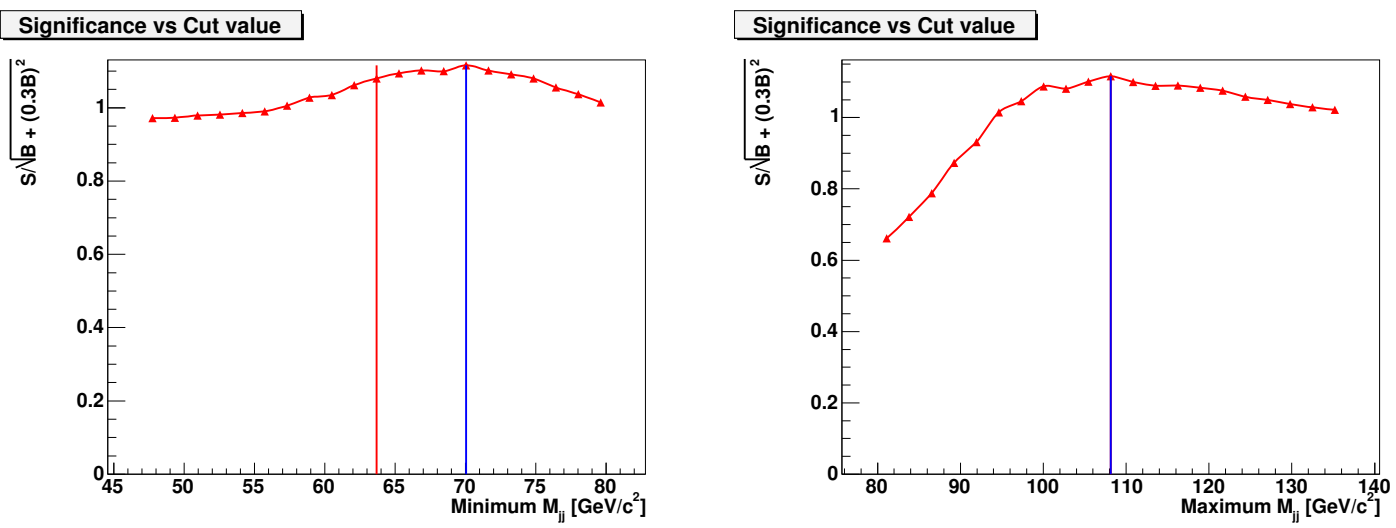

Significance vs Cut value

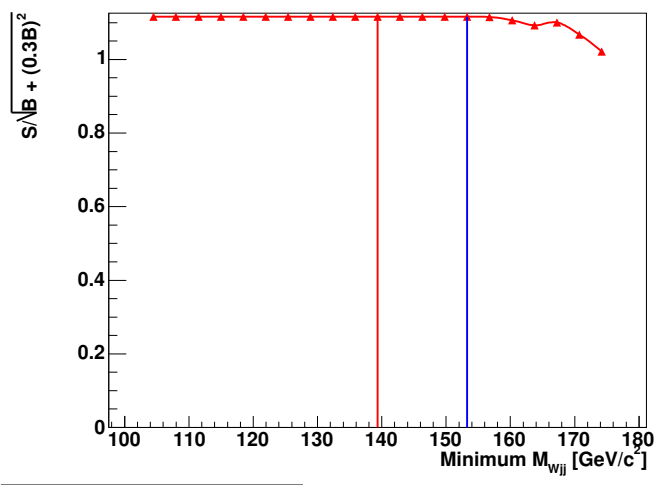

Significance vs Cut value

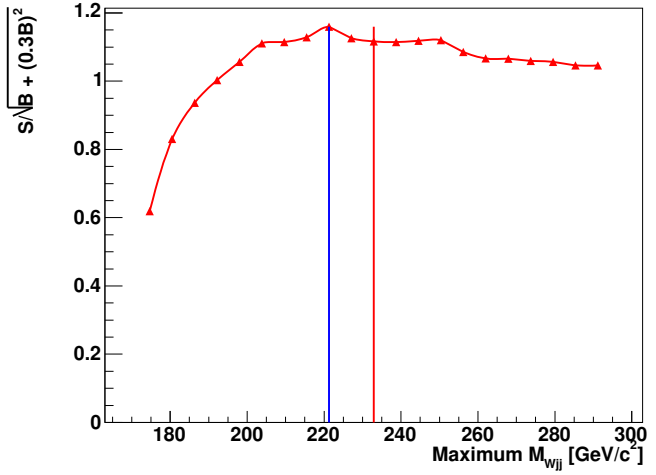

Significance vs Cut value

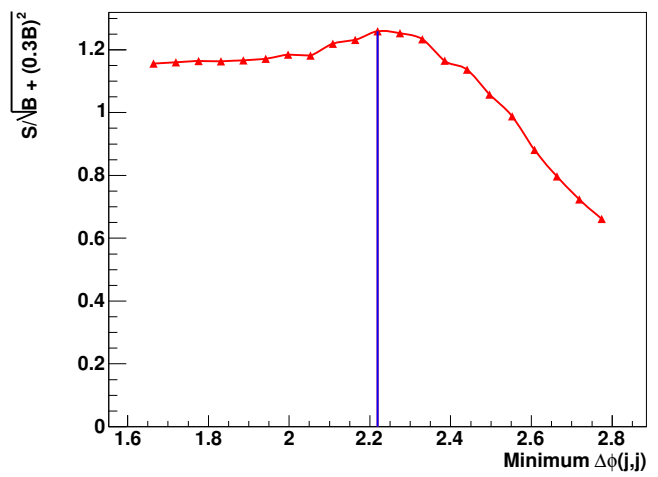

Figure A.5: Results of varying the selection cuts for $M_{\rho}=195 \mathrm{GeV} / c^{2}, M_{\pi}=$ $100 \mathrm{GeV} / c^{2}$. The vertical red line indicates the postion of the initial grid search maximum. The blue line indicates the new maximum after examining the area around the initial maximum. 

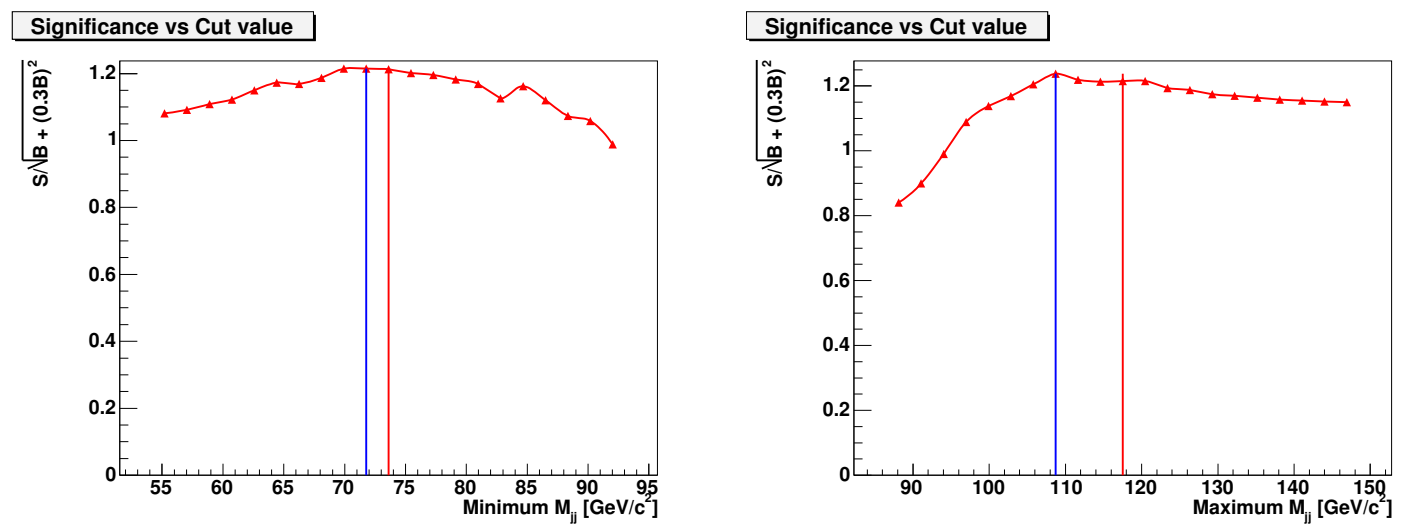

Significance vs Cut value

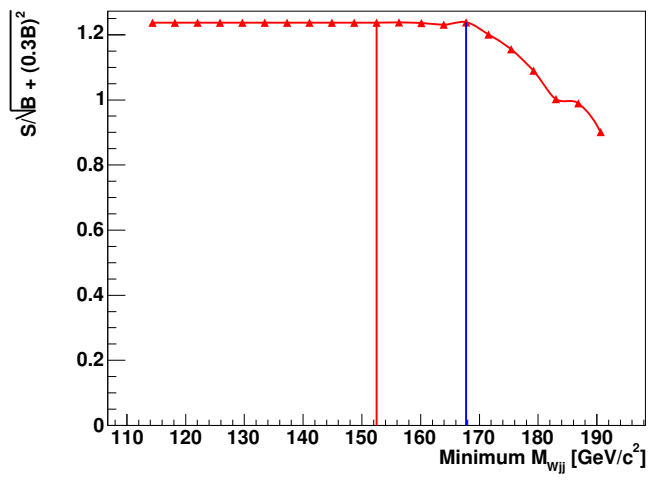

Significance vs Cut value

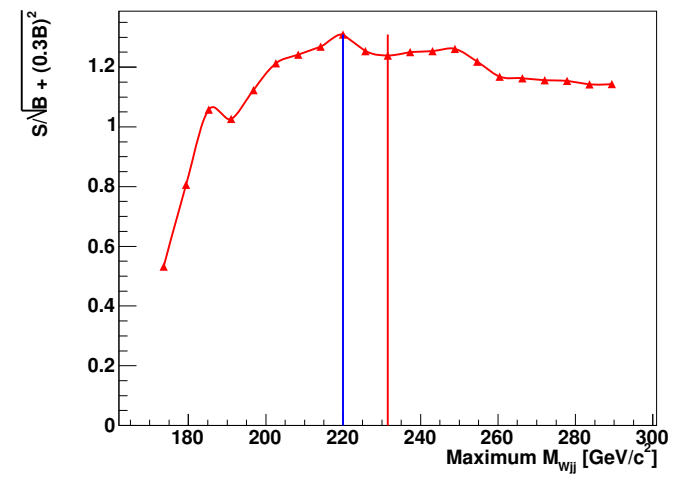

Significance vs Cut value

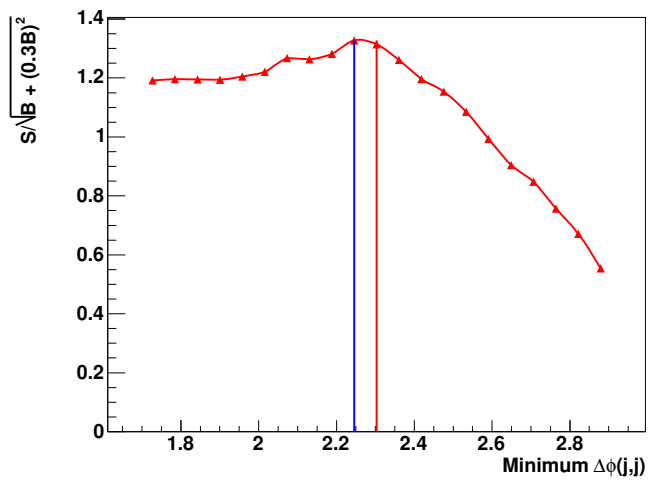

Figure A.6: Results of varying the selection cuts for $M_{\rho}=200 \mathrm{GeV} / c^{2}, M_{\pi}=$ $105 \mathrm{GeV} / c^{2}$. The vertical red line indicates the postion of the initial grid search maximum. The blue line indicates the new maximum after examining the area around the initial maximum. 


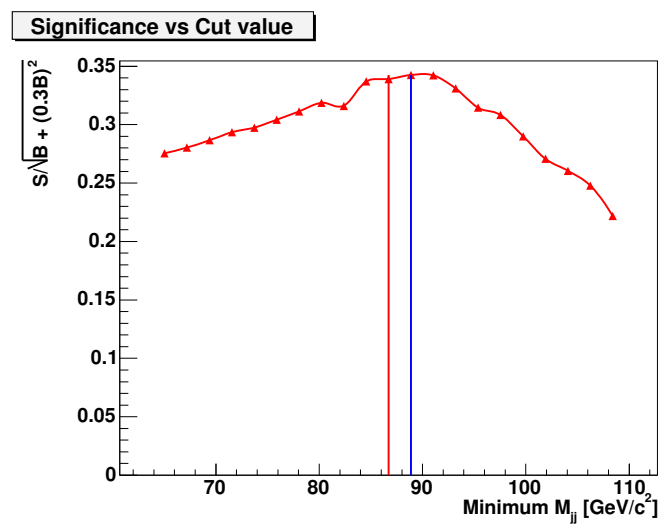

\section{Significance vs Cut value}

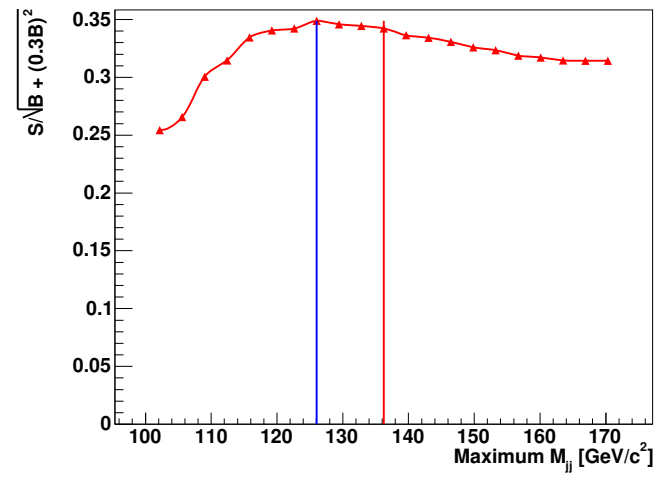

Significance vs Cut value

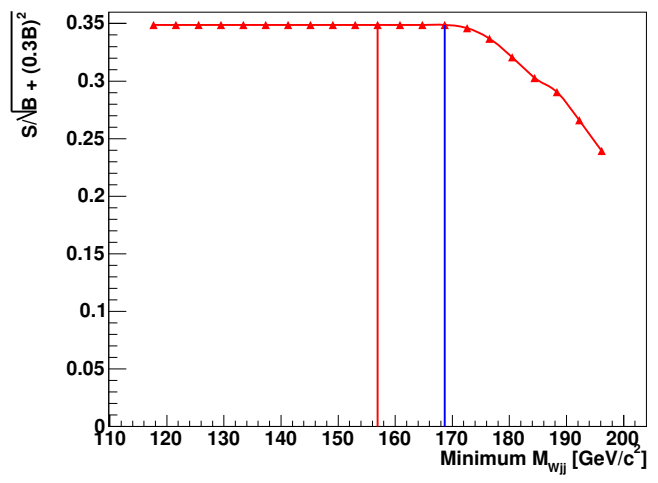

Significance vs Cut value

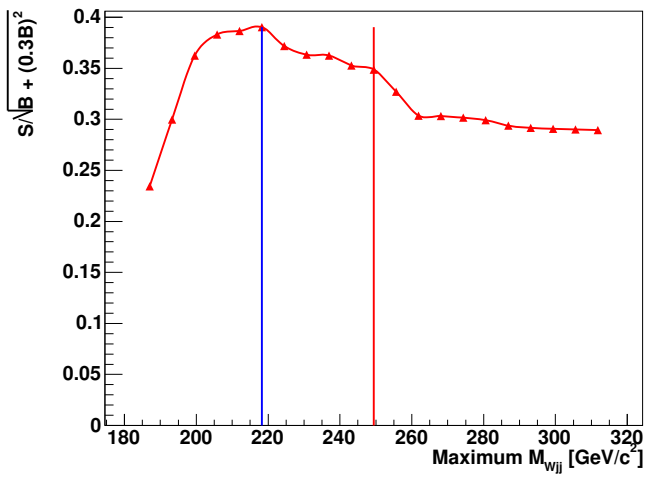

Significance vs Cut value

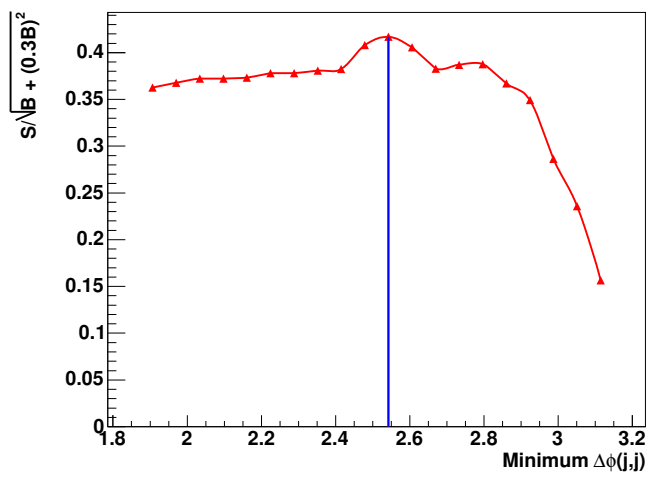

Figure A.7: Results of varying the selection cuts for $M_{\rho}=200 \mathrm{GeV} / c^{2}, M_{\pi}=$ $120 \mathrm{GeV} / c^{2}$. The vertical red line indicates the postion of the initial grid search maximum. The blue line indicates the new maximum after examining the area around the initial maximum. 

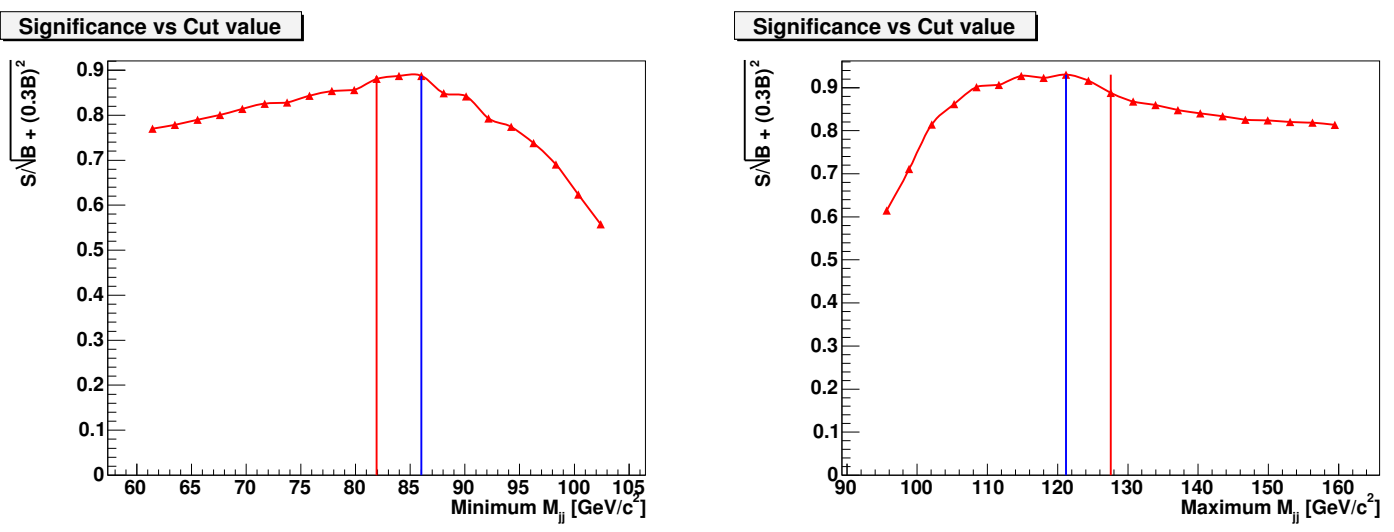

Significance vs Cut value

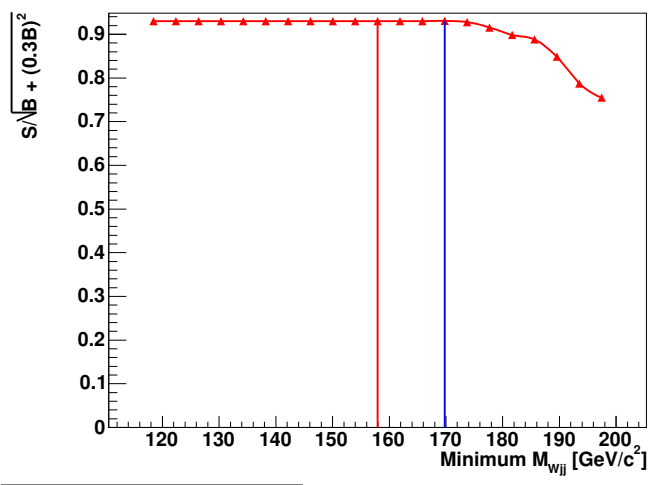

Significance vs Cut value

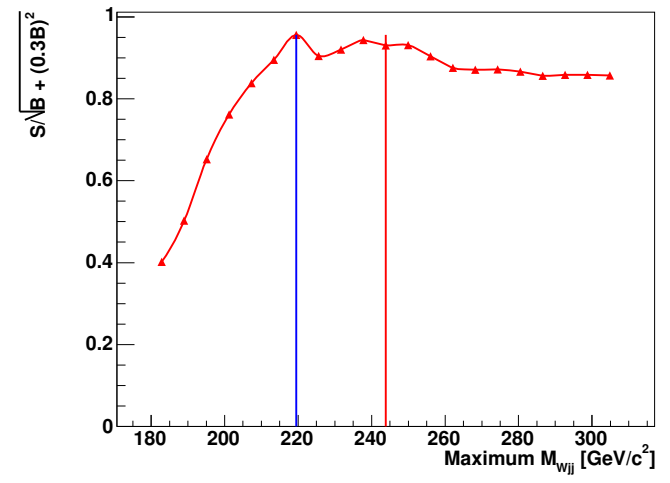

Significance vs Cut value

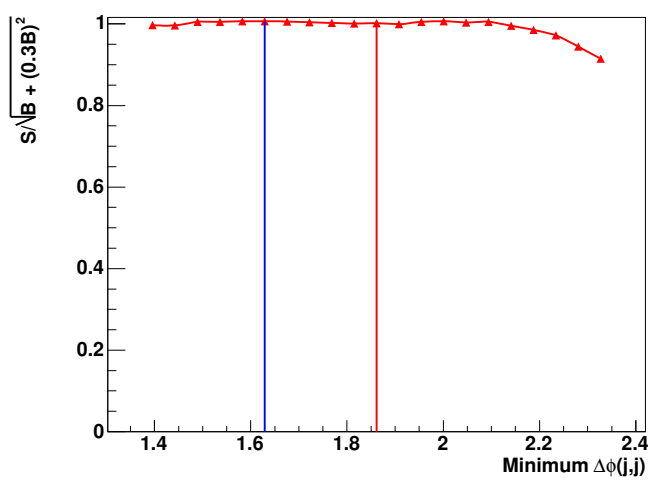

Figure A.8: Results of varying the selection cuts for $M_{\rho}=210 \mathrm{GeV} / c^{2}, M_{\pi}=$ $110 \mathrm{GeV} / c^{2}$. The vertical red line indicates the postion of the initial grid search maximum. The blue line indicates the new maximum after examining the area around the initial maximum. 

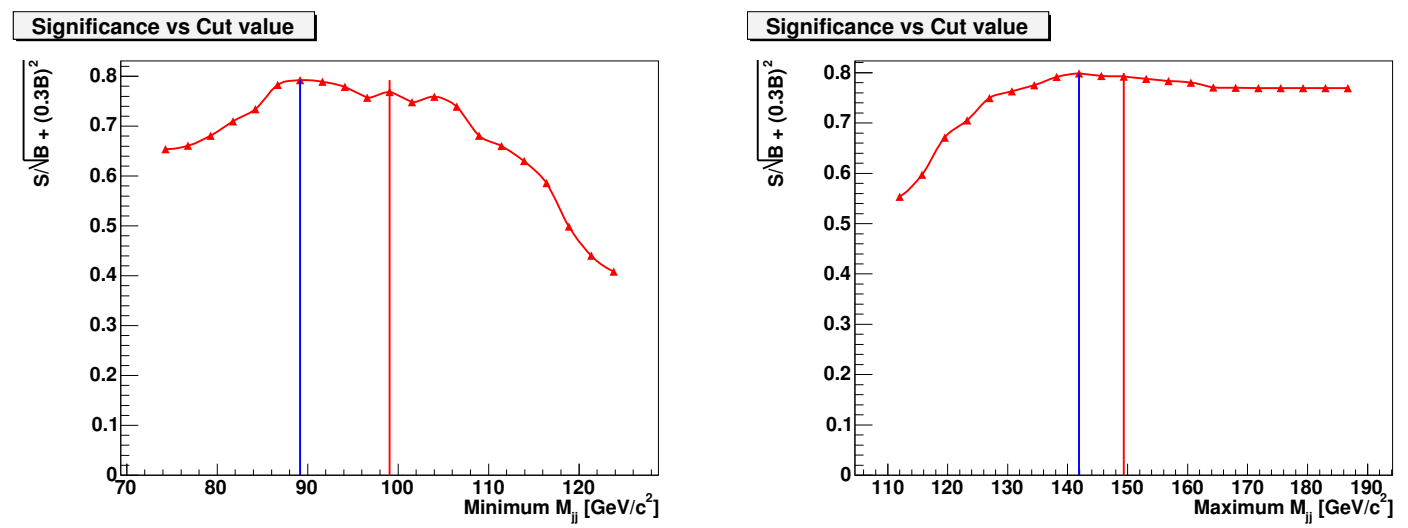

Significance vs Cut value

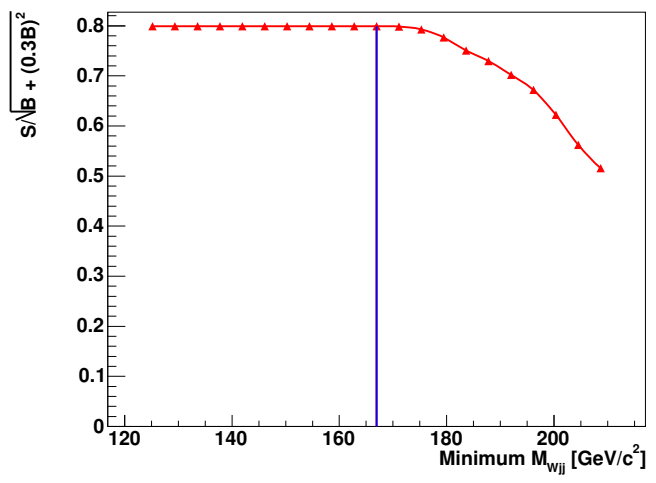

Significance vs Cut value

Significance vs Cut value
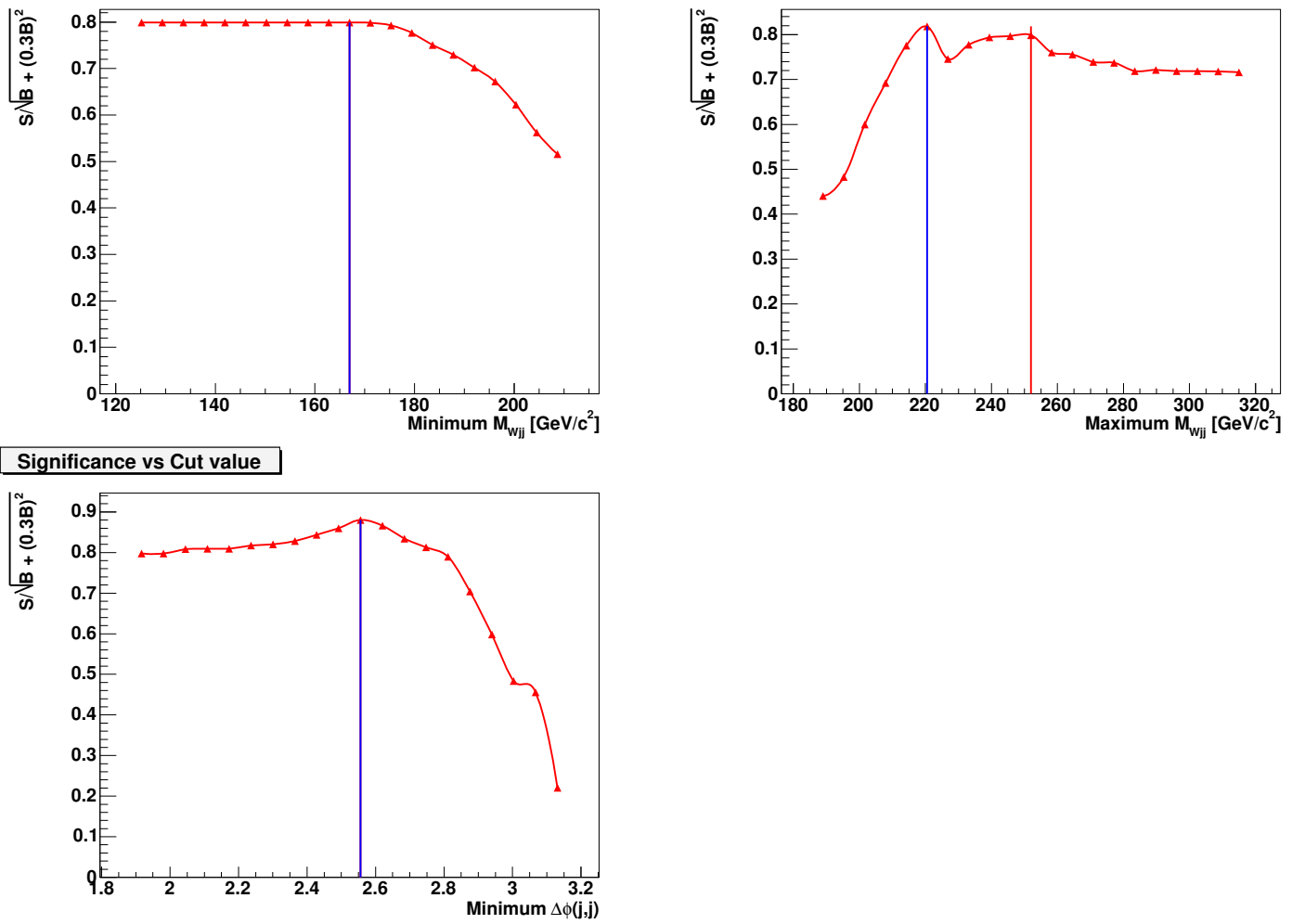

Figure A.9: Results of varying the selection cuts for $M_{\rho}=215 \mathrm{GeV} / c^{2}, M_{\pi}=$ $130 \mathrm{GeV} / c^{2}$. The vertical red line indicates the postion of the initial grid search maximum. The blue line indicates the new maximum after examining the area around the initial maximum. 

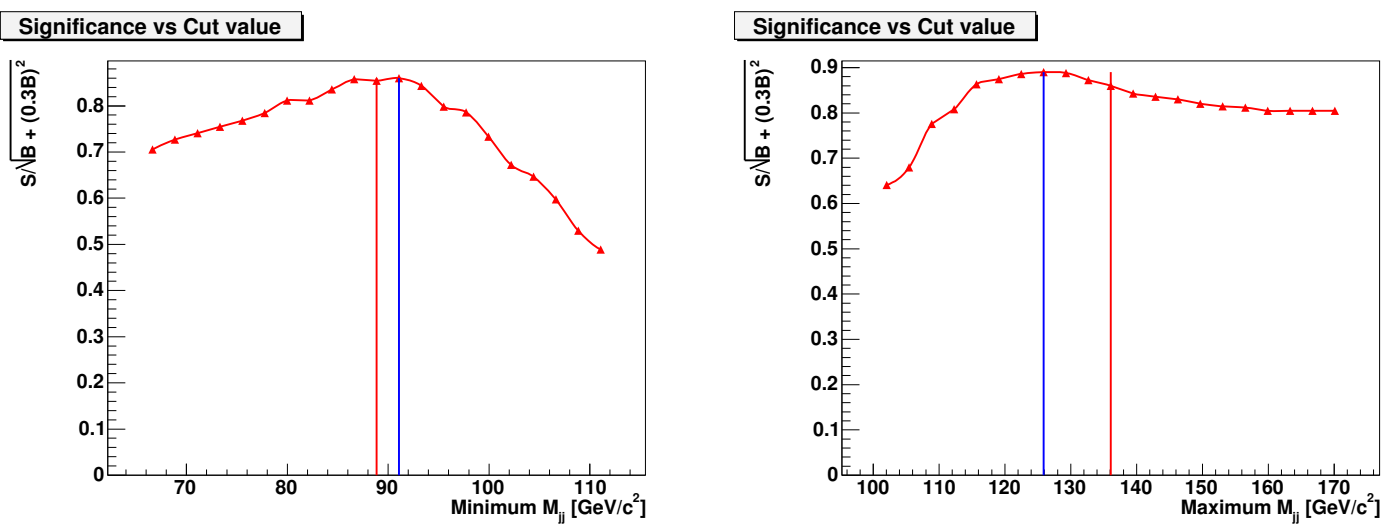

Significance vs Cut value

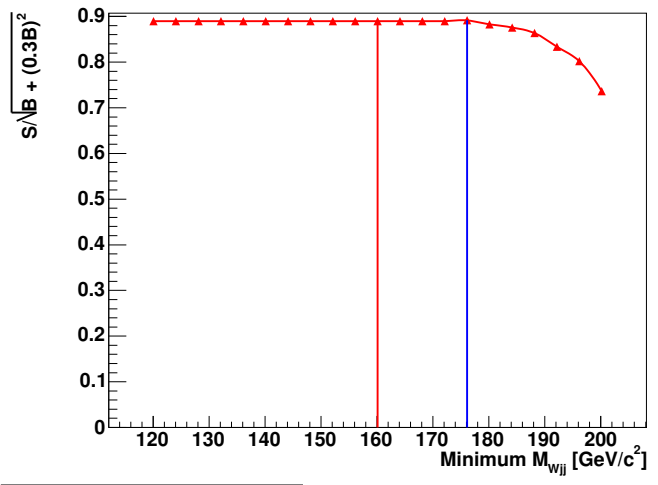

Significance vs Cut value

Significance vs Cut value
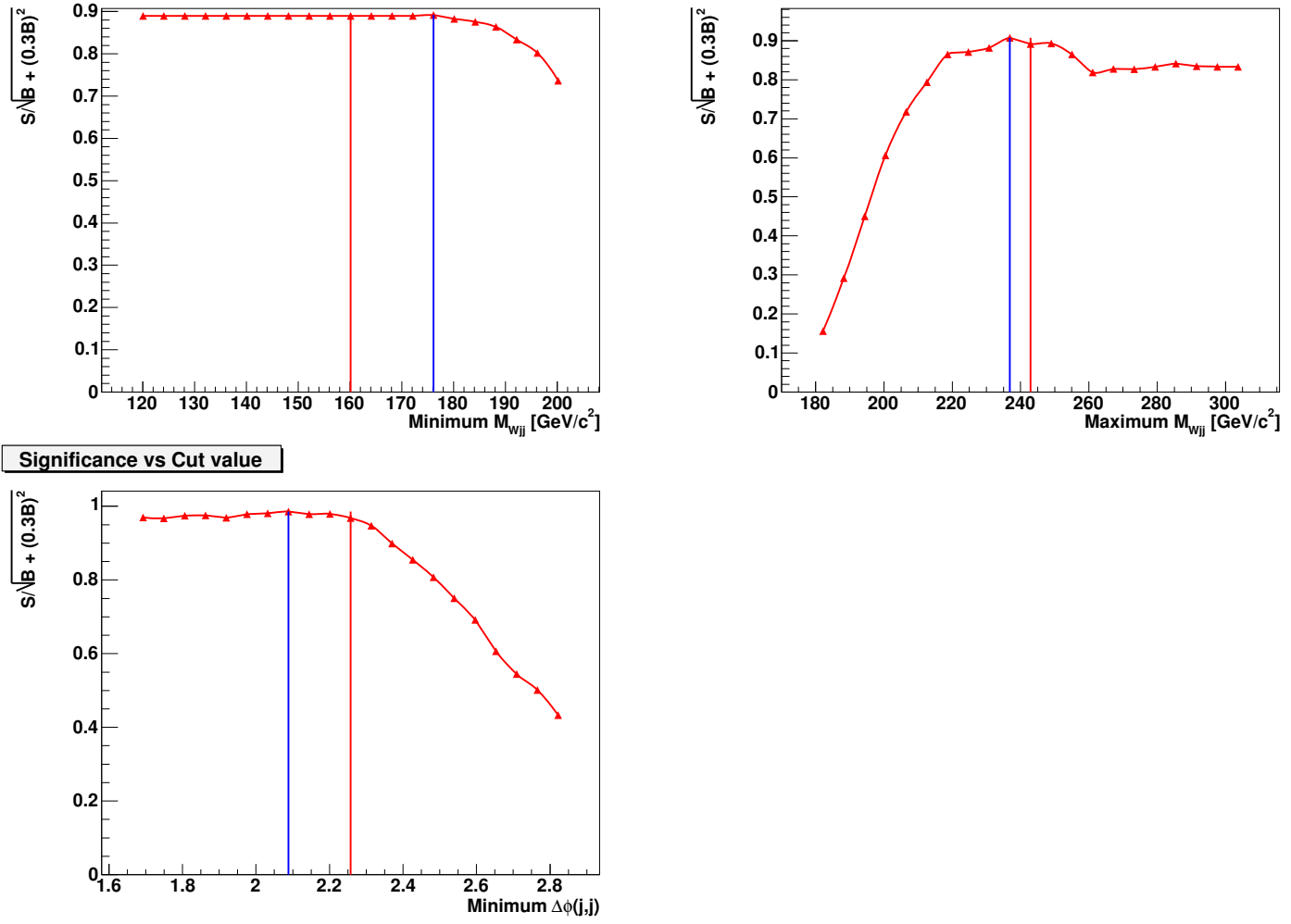

Figure A.10: Results of varying the selection cuts for $M_{\rho}=220 \mathrm{GeV} / c^{2}$, $M_{\pi}=120 \mathrm{GeV} / c^{2}$. The vertical red line indicates the postion of the initial grid search maximum. The blue line indicates the new maximum after examining the area around the initial maximum. 

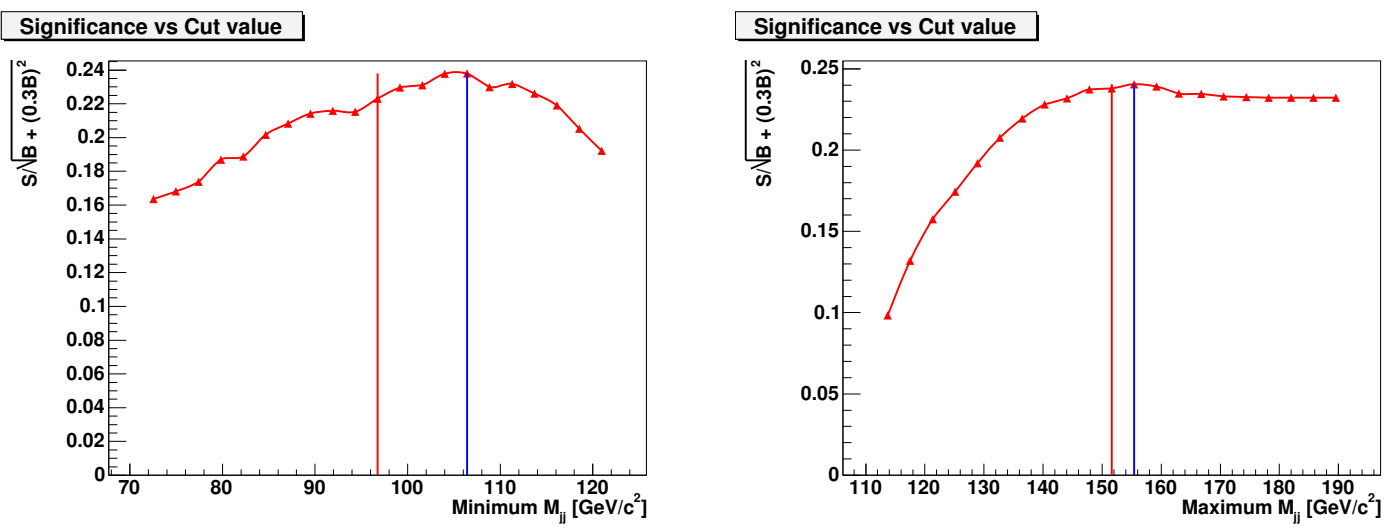

Significance vs Cut value

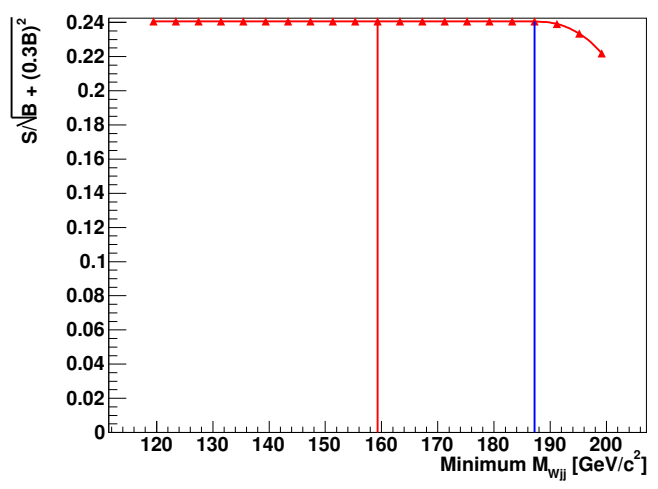

Significance vs Cut value

Significance vs Cut value
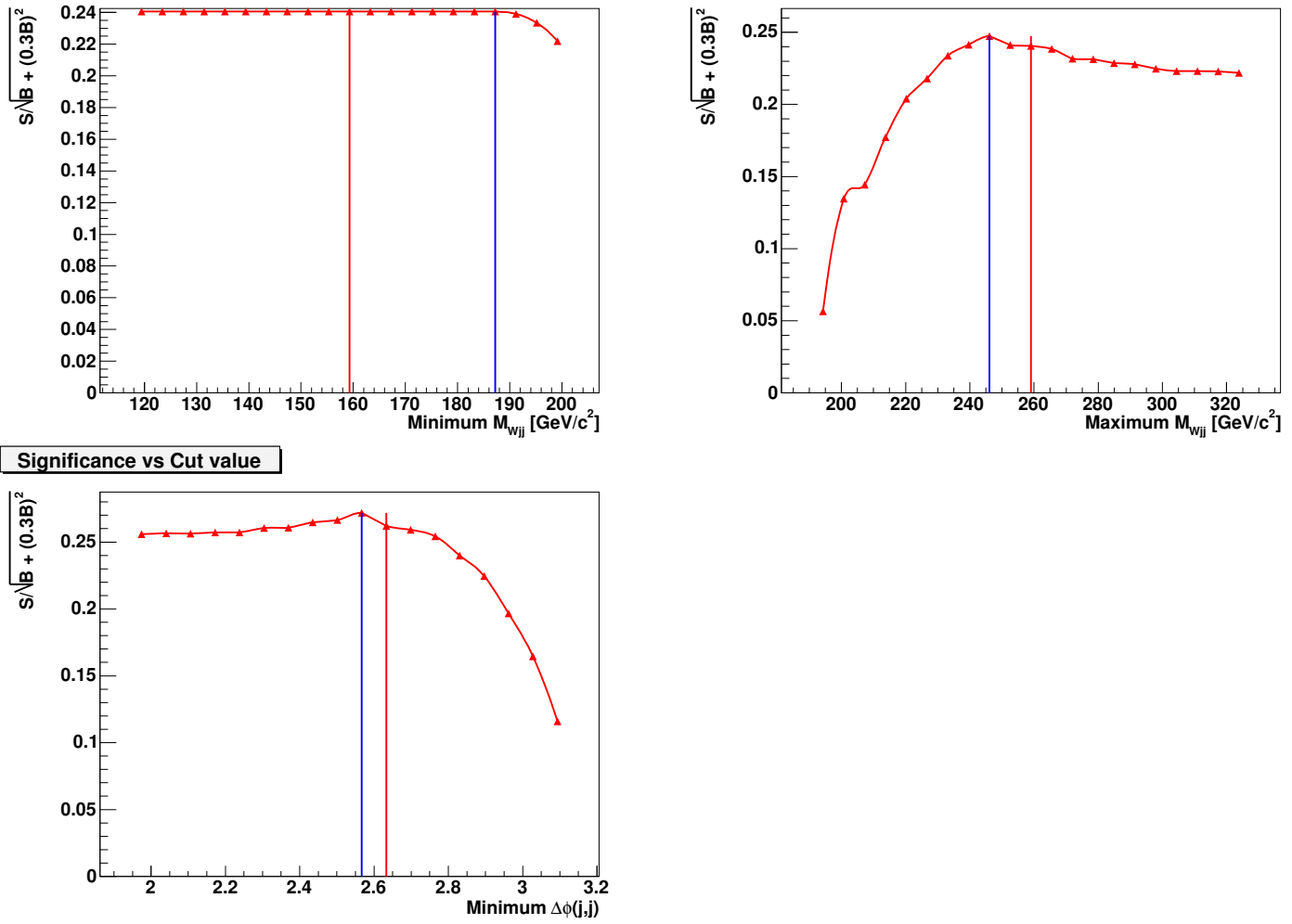

Figure A.11: Results of varying the selection cuts for $M_{\rho}=220 \mathrm{GeV} / c^{2}$, $M_{\pi}=140 \mathrm{GeV} / c^{2}$. The vertical red line indicates the postion of the initial grid search maximum. The blue line indicates the new maximum after examining the area around the initial maximum. 

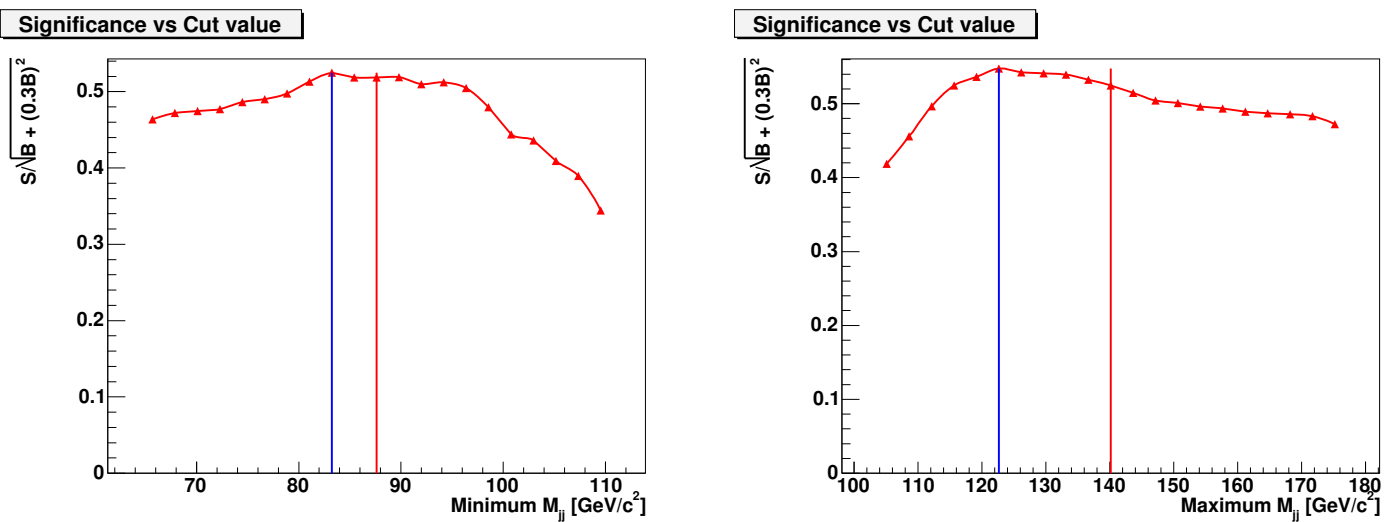

Significance vs Cut value

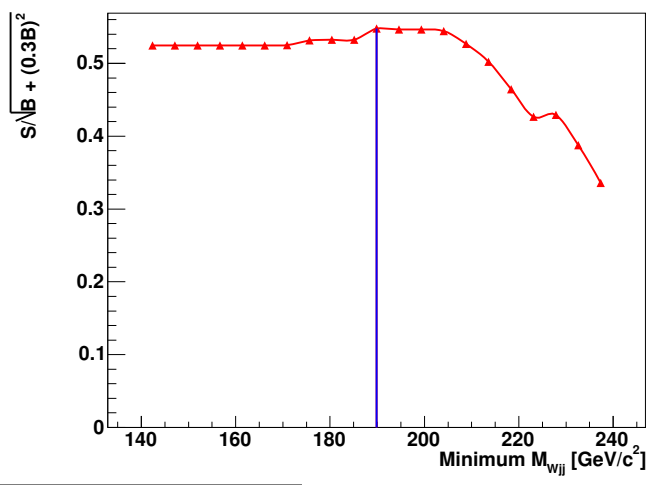

Significance vs Cut value

Significance vs Cut value
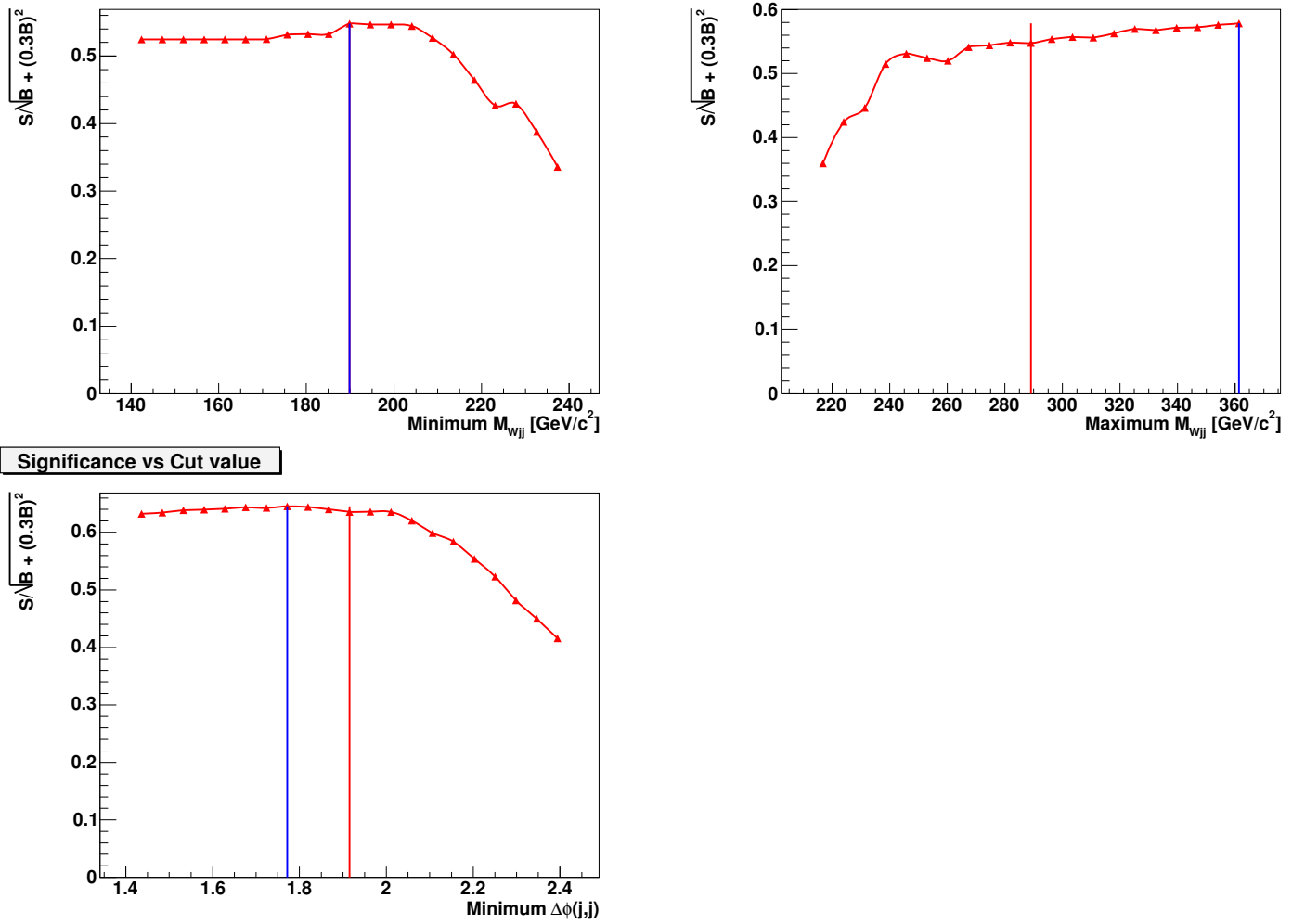

Figure A.12: Results of varying the selection cuts for $M_{\rho}=235 \mathrm{GeV} / c^{2}$, $M_{\pi}=120 \mathrm{GeV} / c^{2}$. The vertical red line indicates the postion of the initial grid search maximum. The blue line indicates the new maximum after examining the area around the initial maximum. 

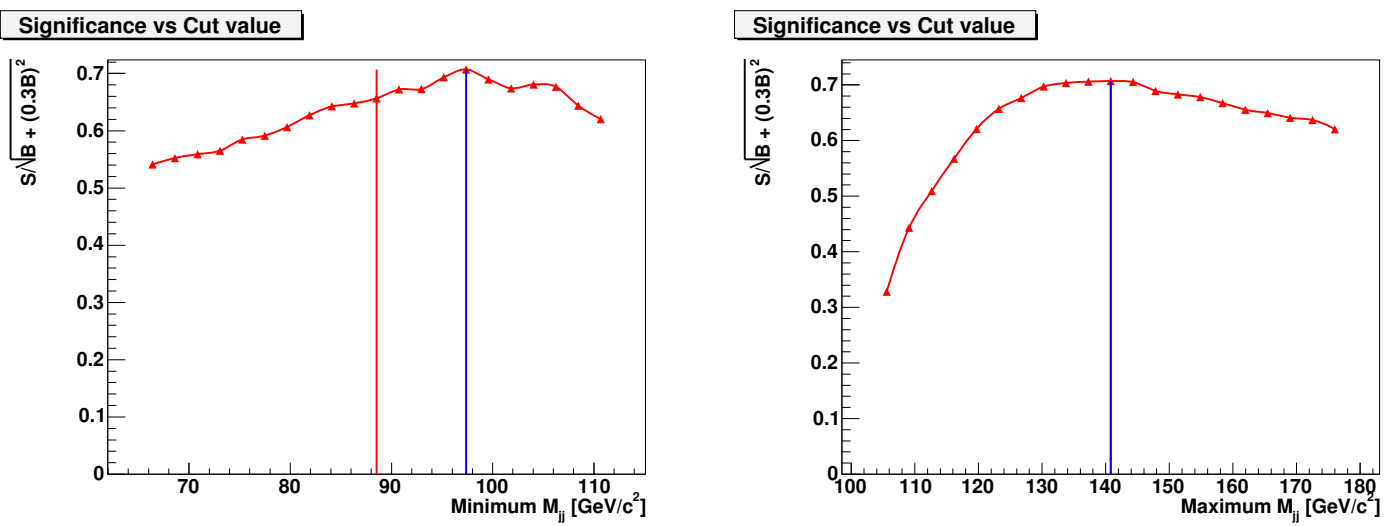

Significance vs Cut value

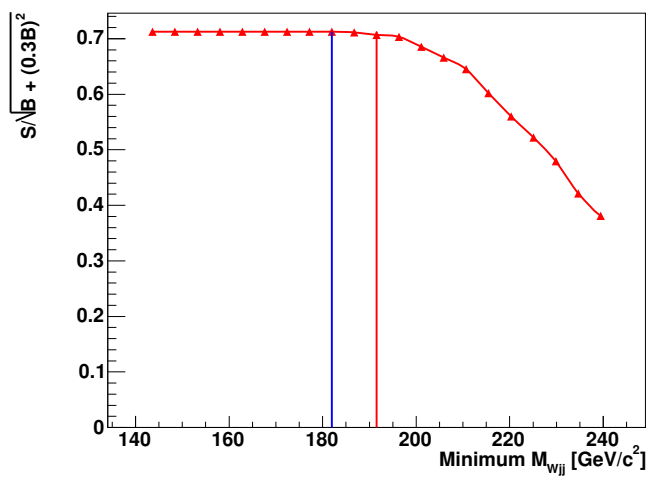

Significance vs Cut value

Significance vs Cut value
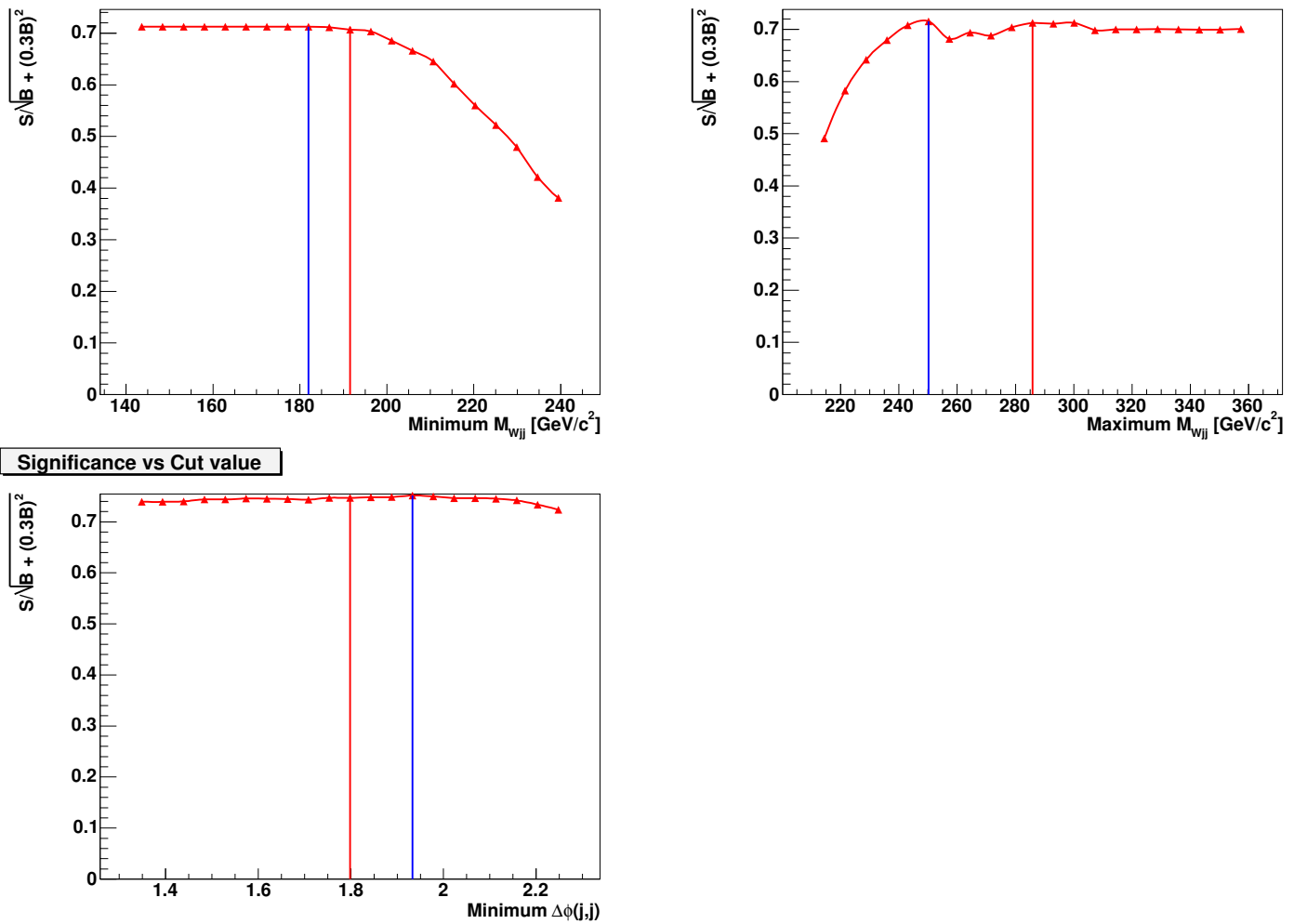

Figure A.13: Results of varying the selection cuts for $M_{\rho}=235 \mathrm{GeV} / c^{2}$, $M_{\pi}=130 \mathrm{GeV} / c^{2}$. The vertical red line indicates the postion of the initial grid search maximum. The blue line indicates the new maximum after examining the area around the initial maximum. 

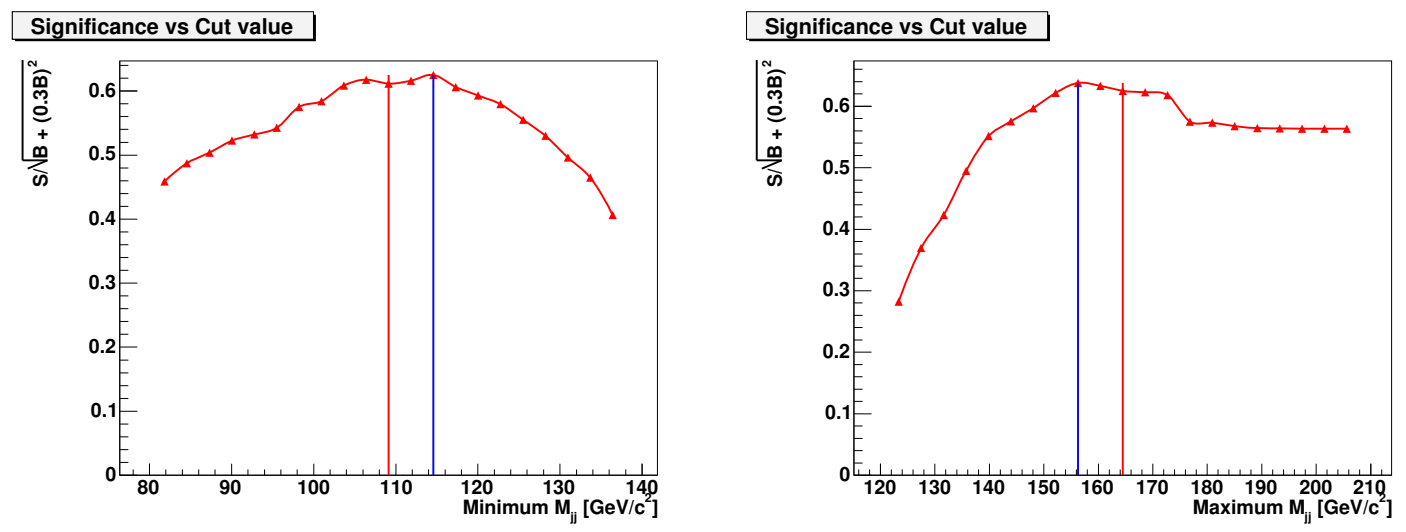

Significance vs Cut value

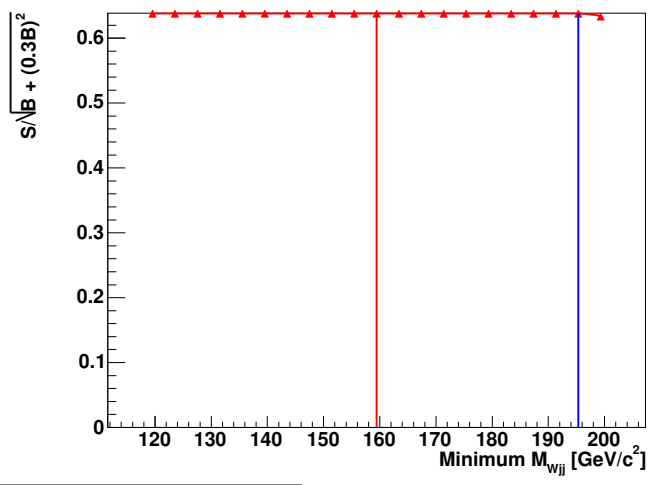

Significance vs Cut value

Significance vs Cut value
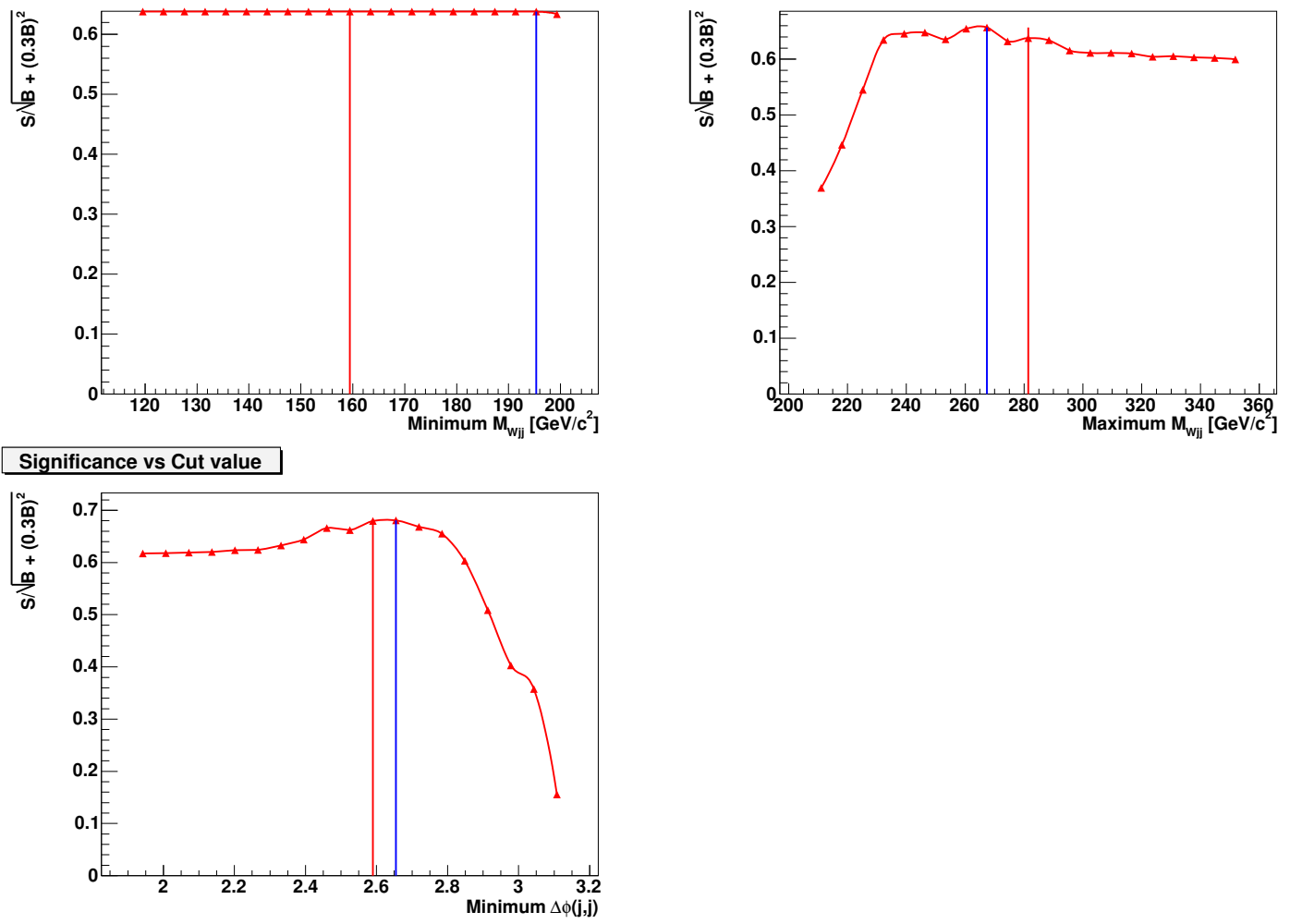

Figure A.14: Results of varying the selection cuts for $M_{\rho}=235 \mathrm{GeV} / c^{2}$, $M_{\pi}=150 \mathrm{GeV} / c^{2}$. The vertical red line indicates the postion of the initial grid search maximum. The blue line indicates the new maximum after examining the area around the initial maximum. 

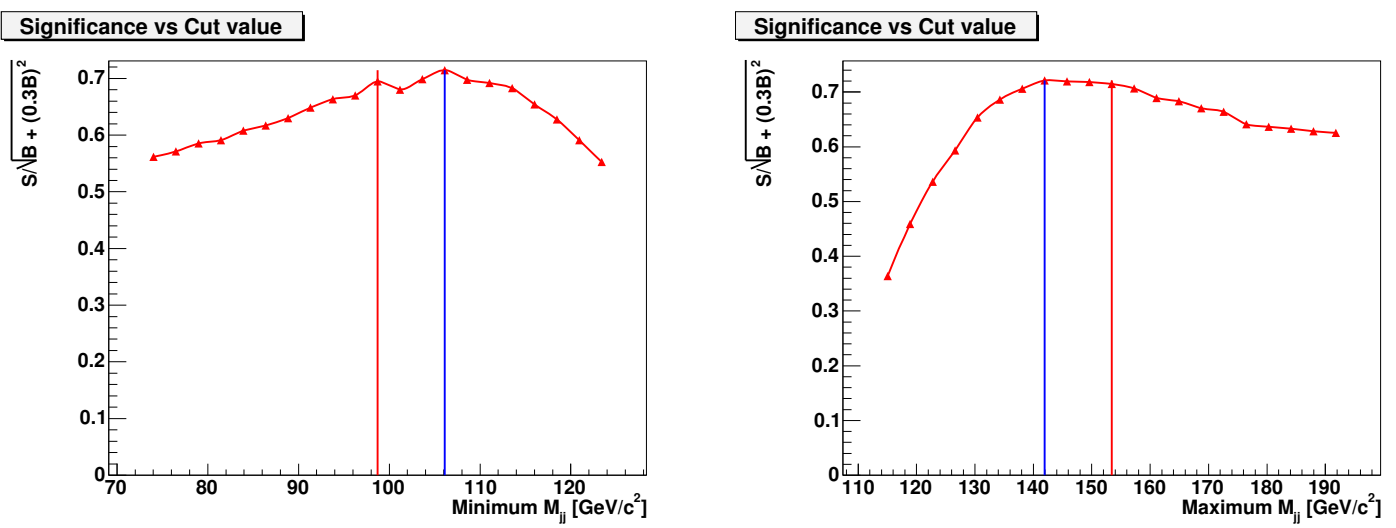

Significance vs Cut value

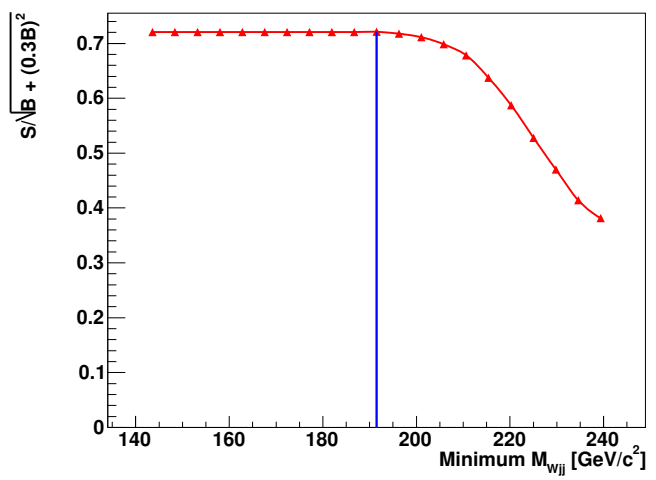

Significance vs Cut value

Significance vs Cut value
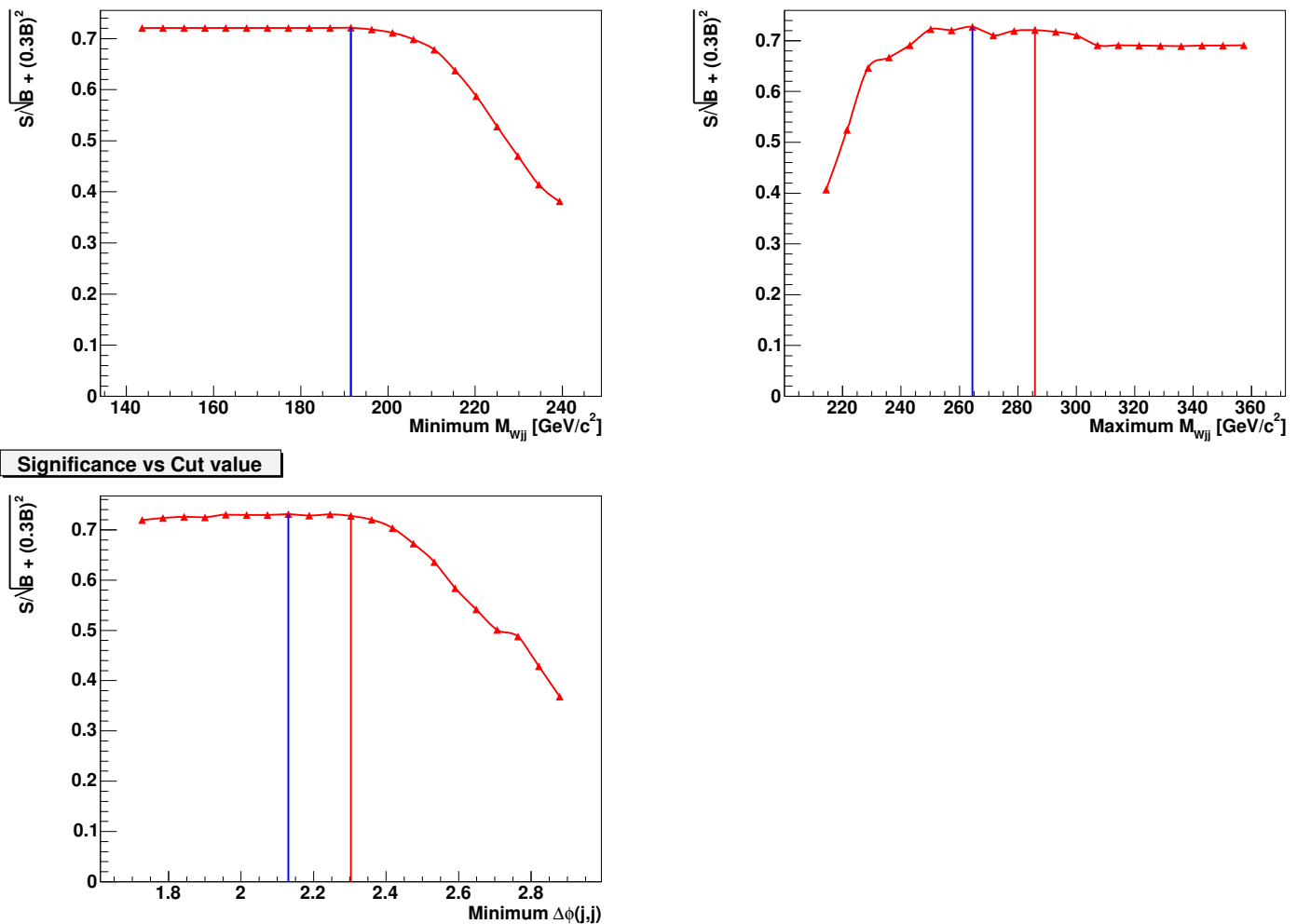

Figure A.15: Results of varying the selection cuts for $M_{\rho}=240 \mathrm{GeV} / c^{2}$, $M_{\pi}=140 \mathrm{GeV} / c^{2}$. The vertical red line indicates the postion of the initial grid search maximum. The blue line indicates the new maximum after examining the area around the initial maximum. 

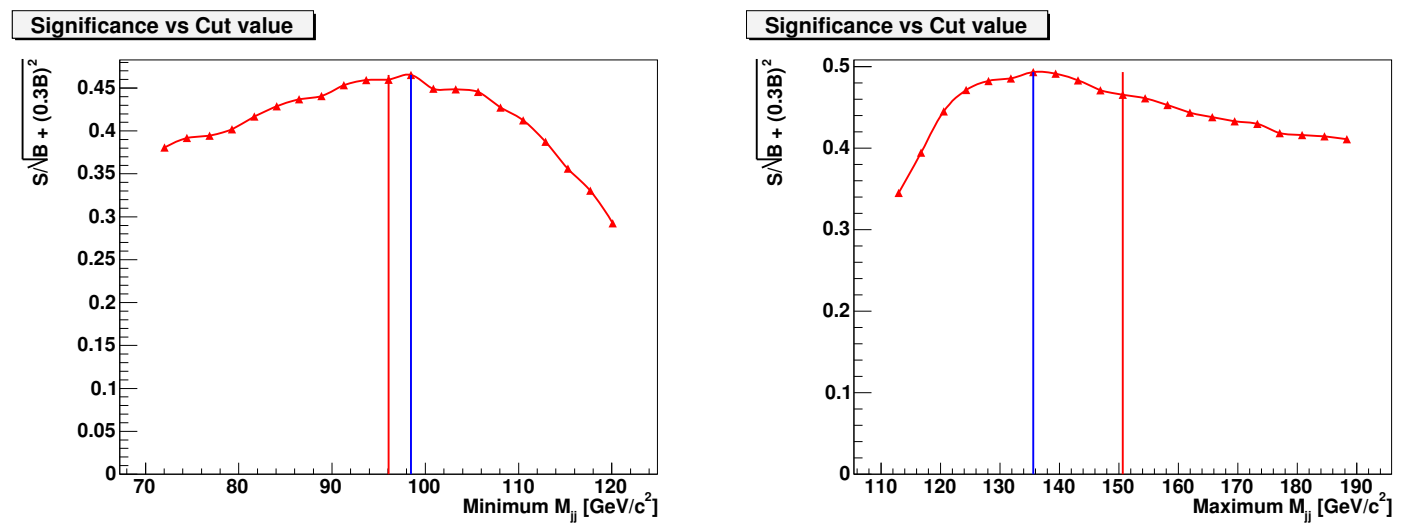

Significance vs Cut value

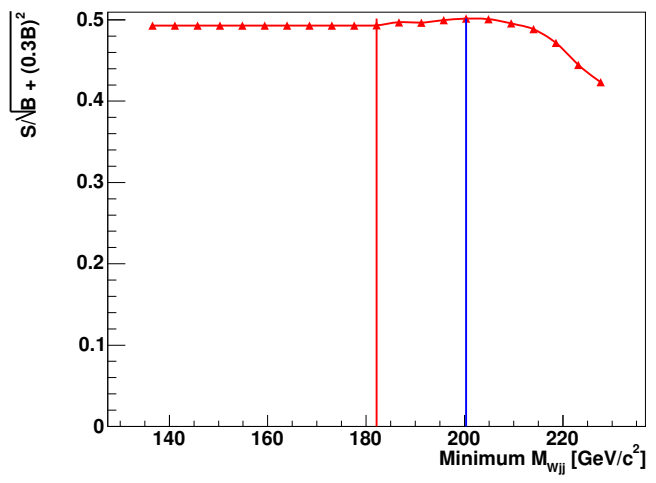

Significance vs Cut value

Significance vs Cut value
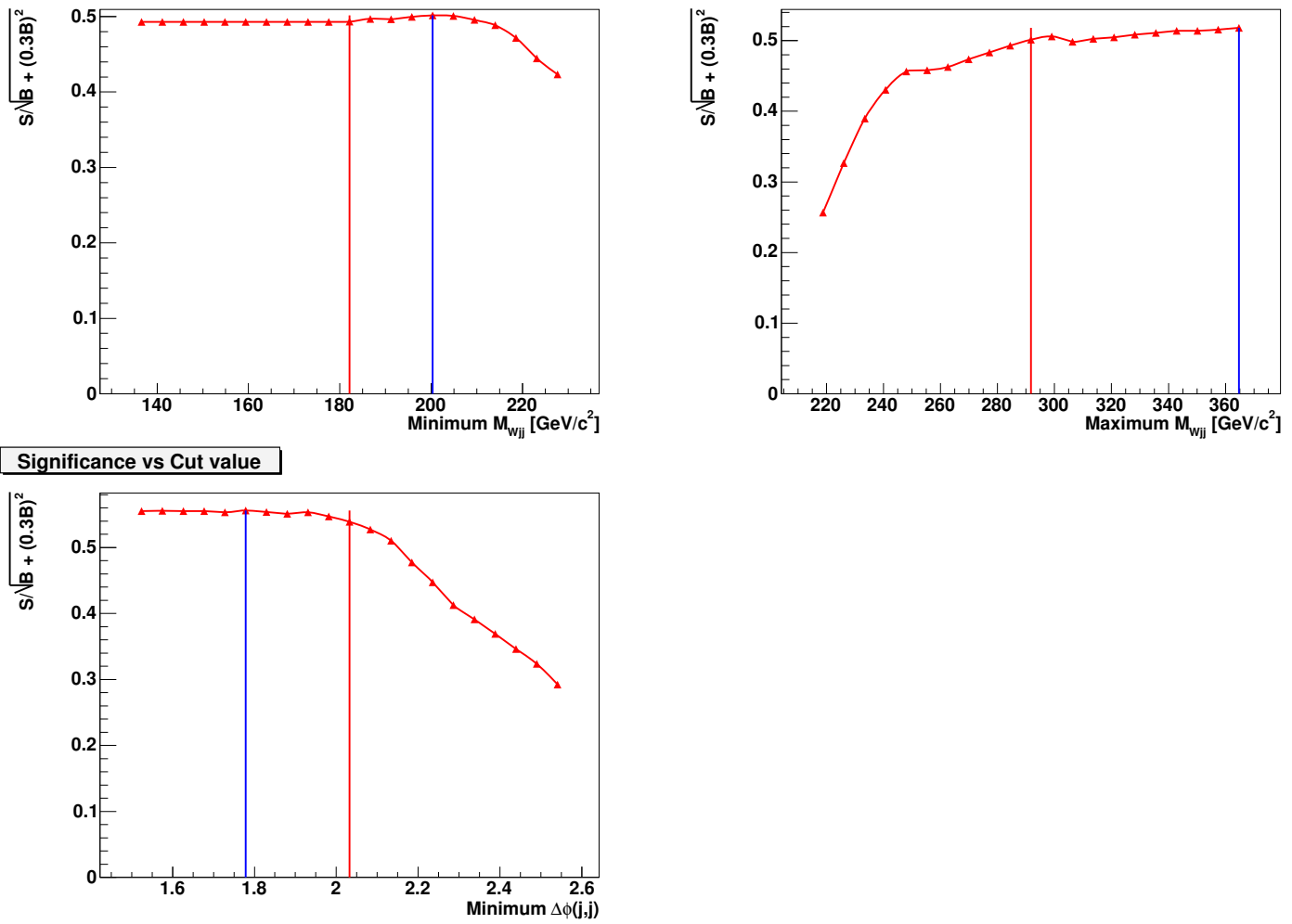

Figure A.16: Results of varying the selection cuts for $M_{\rho}=250 \mathrm{GeV} / c^{2}$, $M_{\pi}=130 \mathrm{GeV} / c^{2}$. The vertical red line indicates the postion of the initial grid search maximum. The blue line indicates the new maximum after examining the area around the initial maximum. 

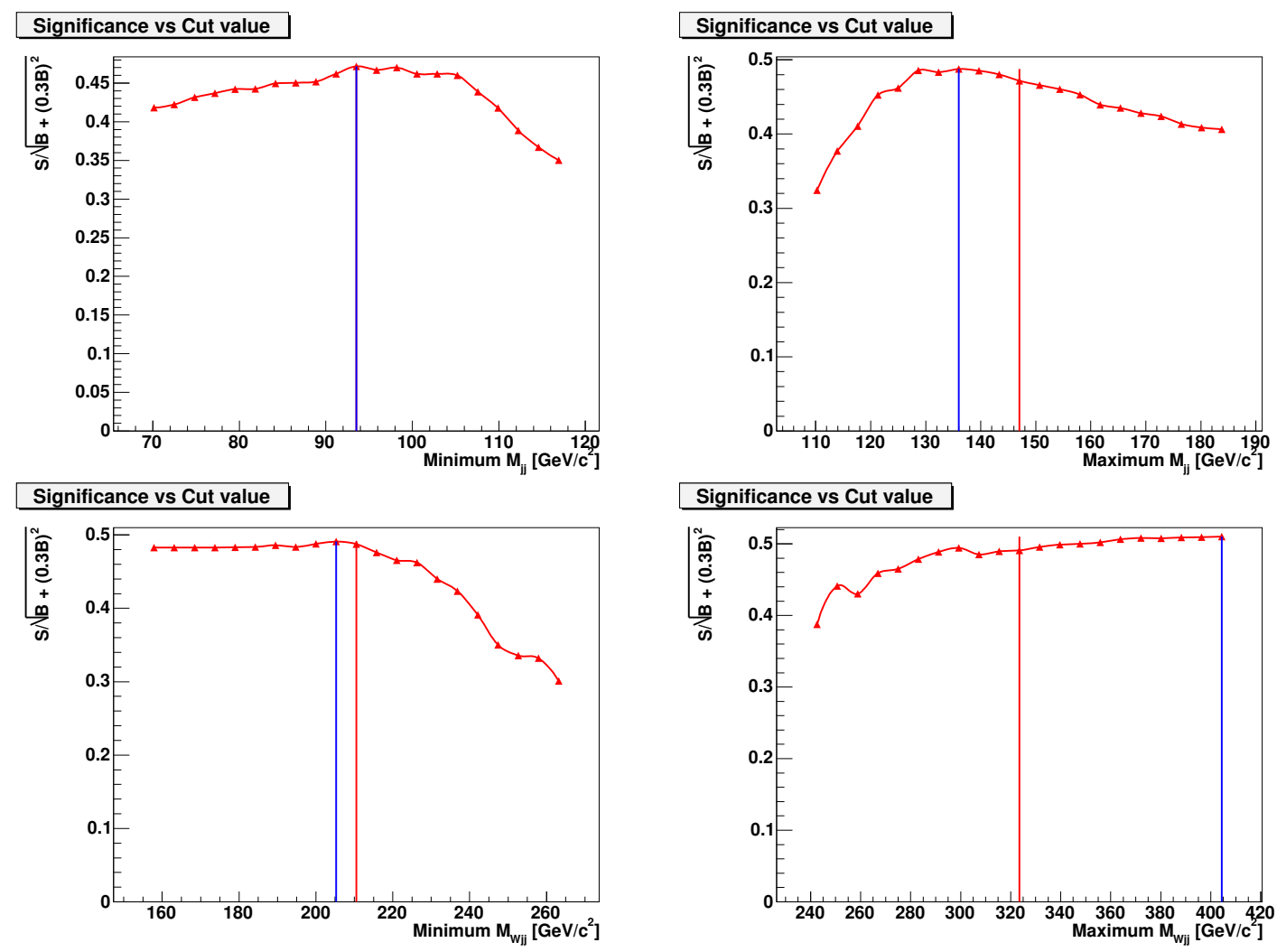

Significance vs Cut value

Significance vs Cut value
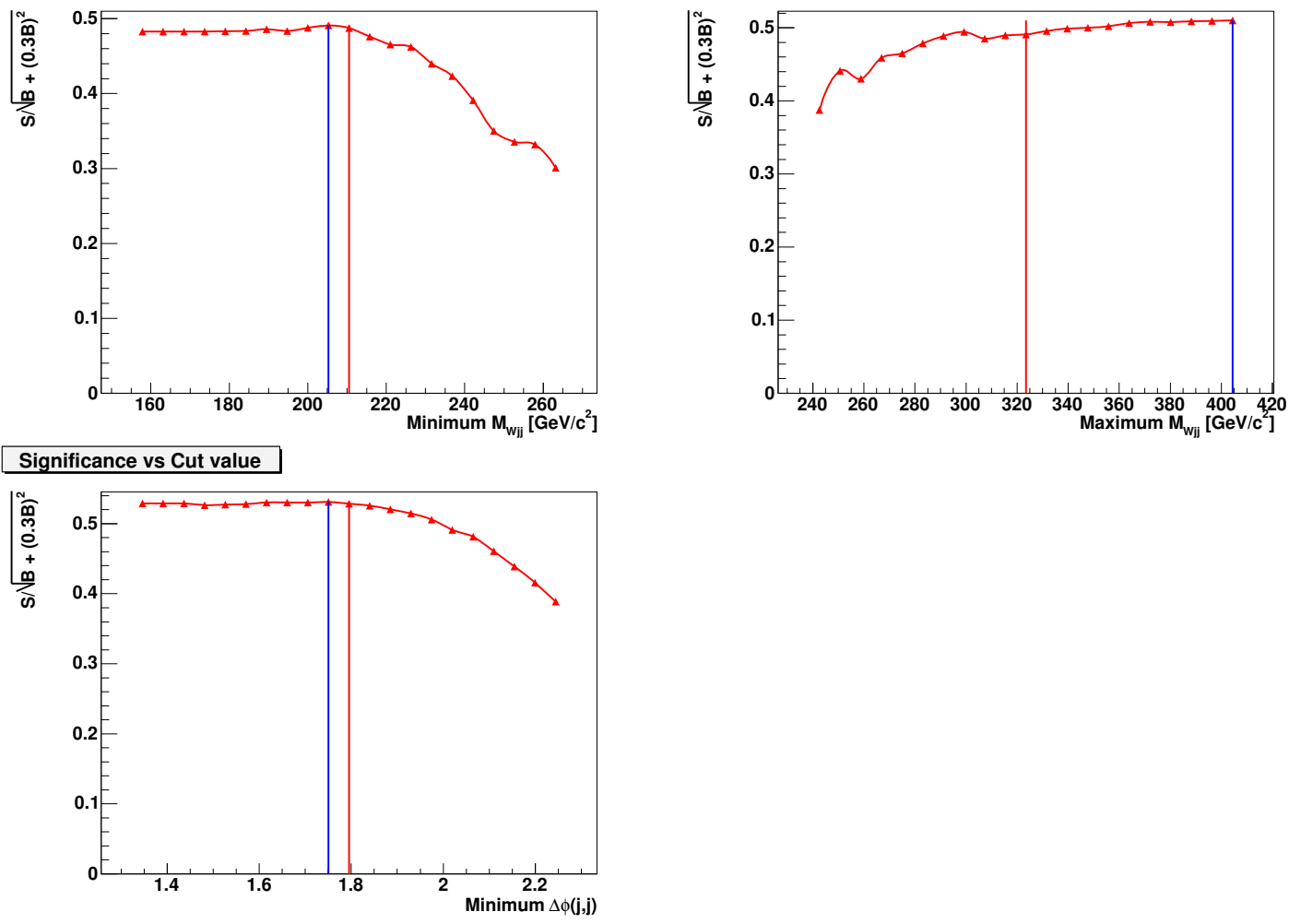

Figure A.17: Results of varying the selection cuts for $M_{\rho}=255 \mathrm{GeV} / c^{2}$, $M_{\pi}=130 \mathrm{GeV} / c^{2}$. The vertical red line indicates the postion of the initial grid search maximum. The blue line indicates the new maximum after examining the area around the initial maximum. 

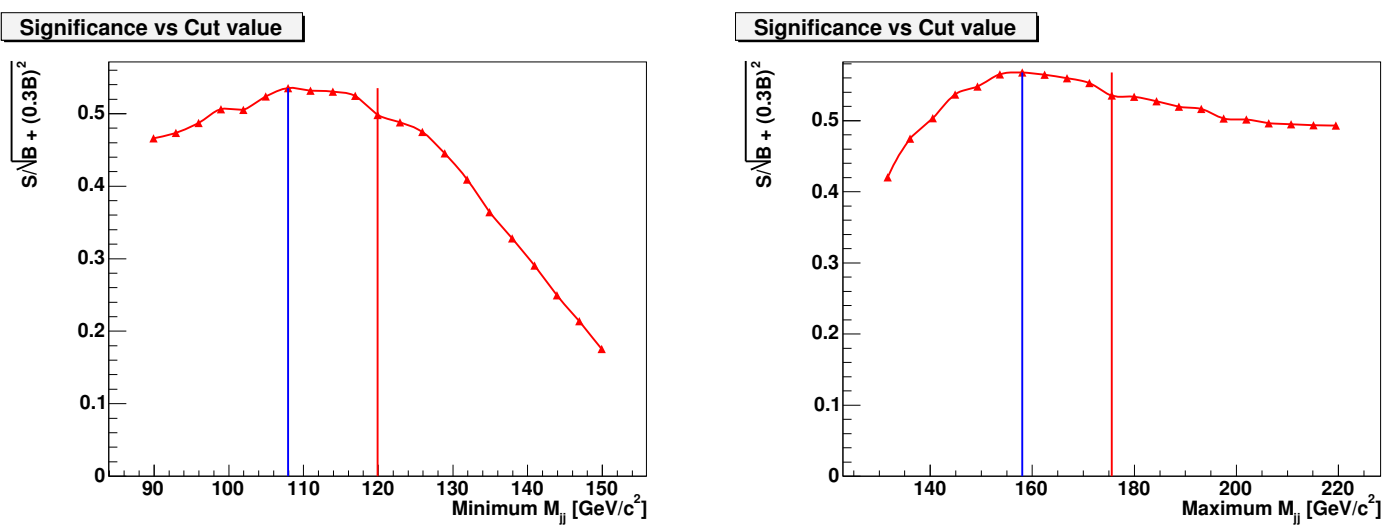

Significance vs Cut value

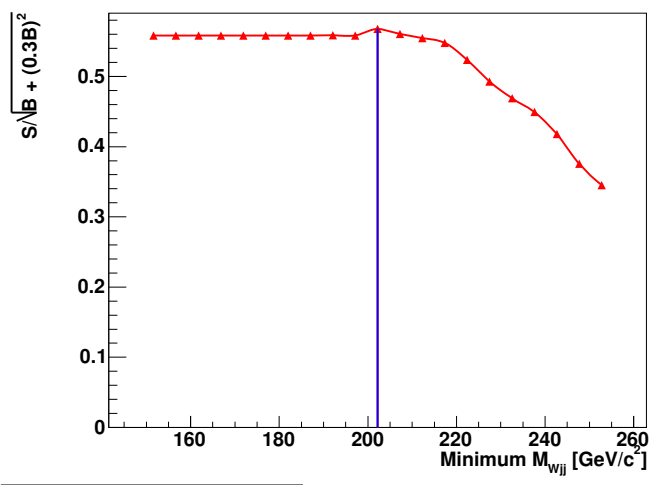

Significance vs Cut value

Significance vs Cut value
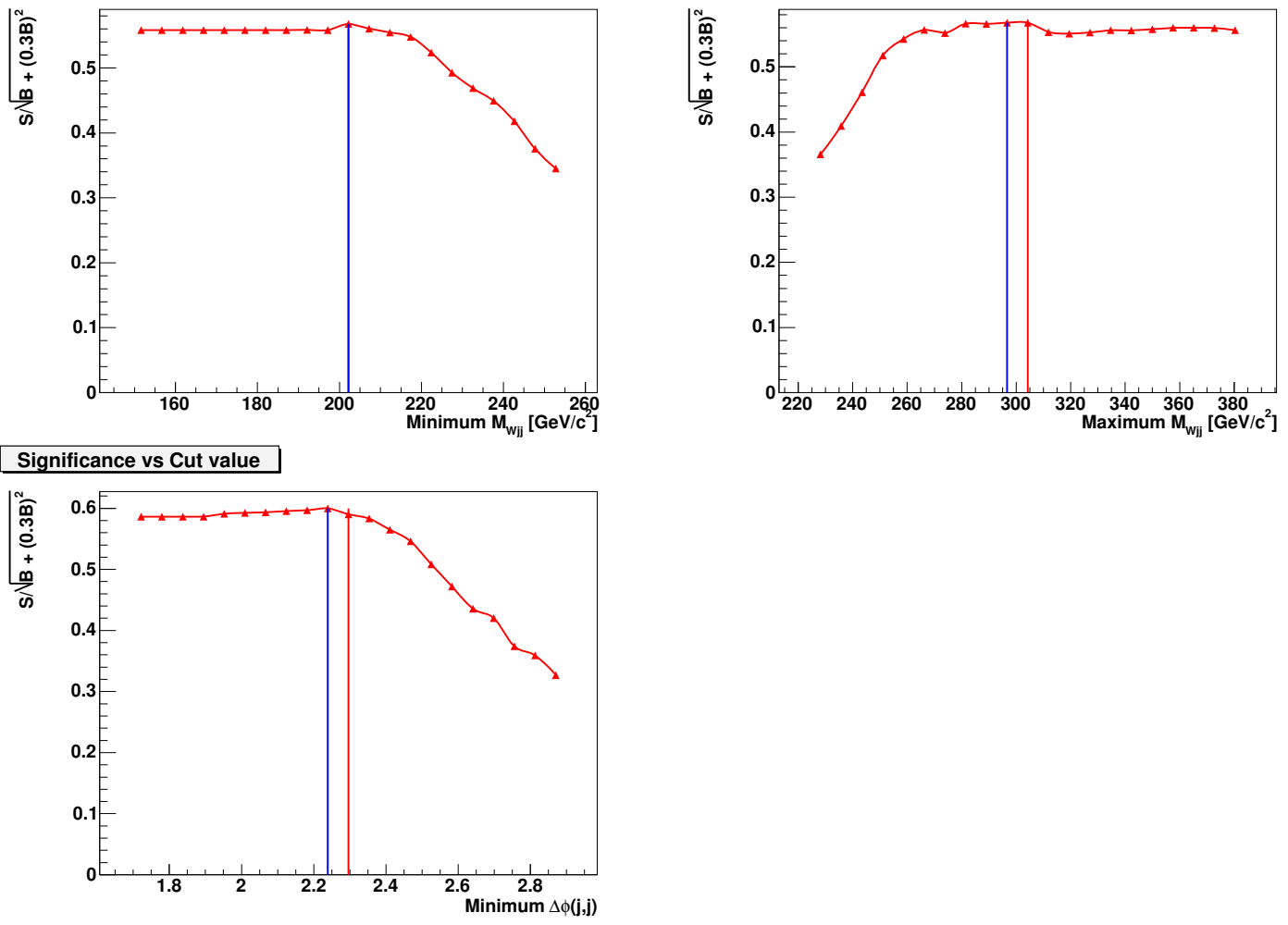

Figure A.18: Results of varying the selection cuts for $M_{\rho}=255 \mathrm{GeV} / c^{2}$, $M_{\pi}=150 \mathrm{GeV} / c^{2}$. The vertical red line indicates the postion of the initial grid search maximum. The blue line indicates the new maximum after examining the area around the initial maximum. 

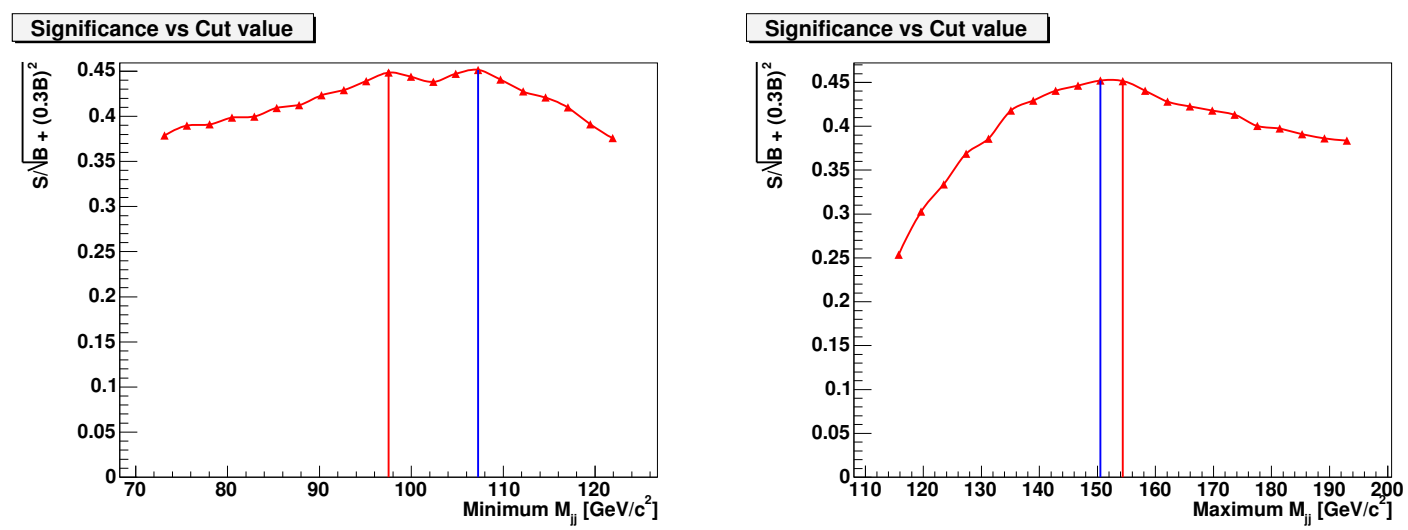

Significance vs Cut value

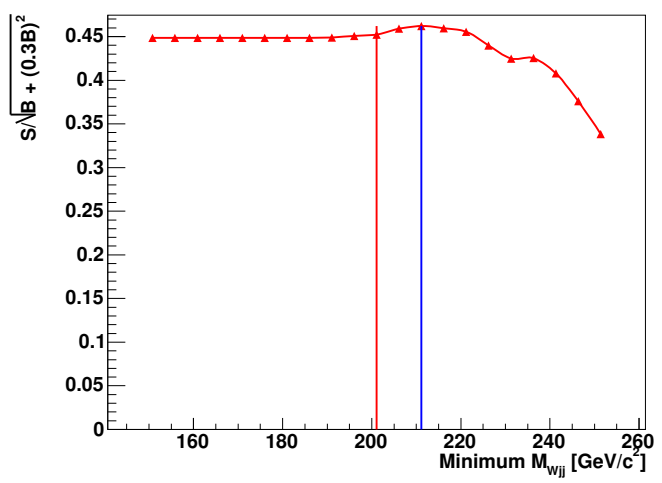

Significance vs Cut value

Significance vs Cut value
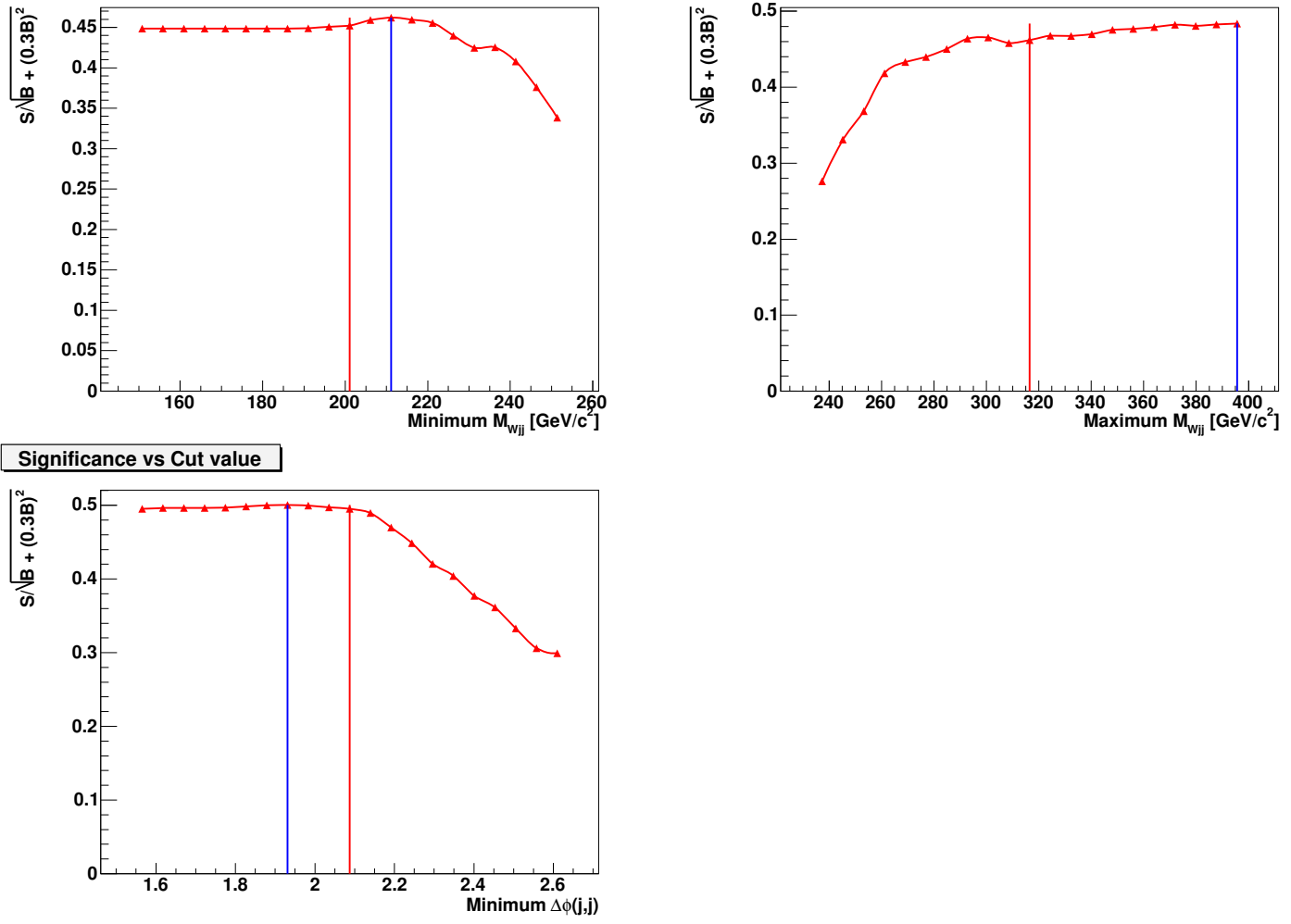

Figure A.19: Results of varying the selection cuts for $M_{\rho}=260 \mathrm{GeV} / c^{2}$, $M_{\pi}=140 \mathrm{GeV} / c^{2}$. The vertical red line indicates the postion of the initial grid search maximum. The blue line indicates the new maximum after examining the area around the initial maximum. 

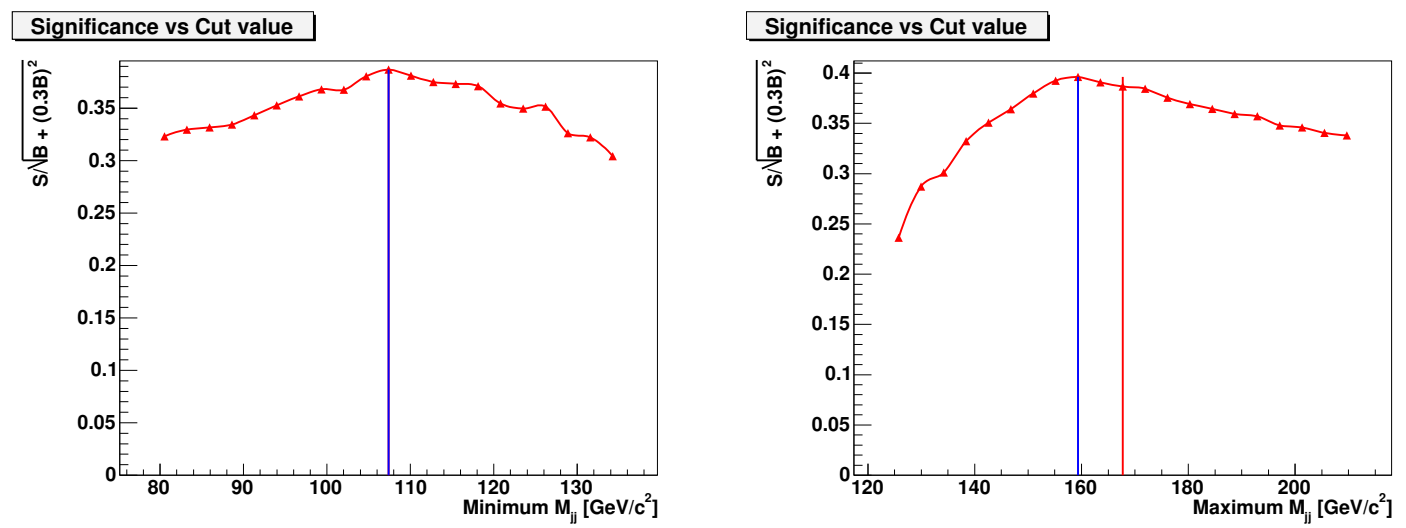

Significance vs Cut value

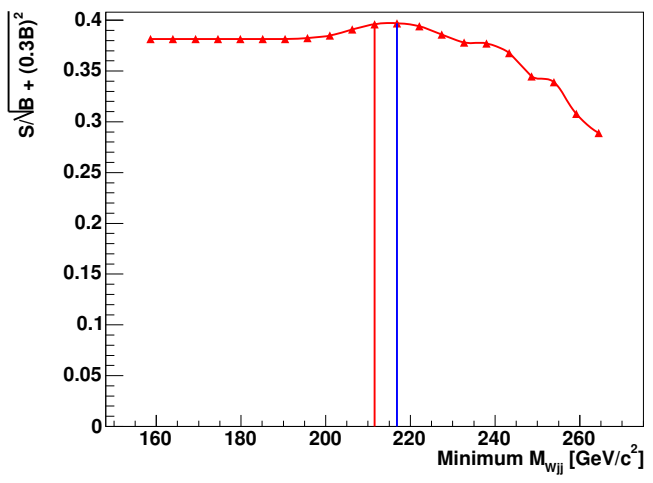

Significance vs Cut value

Significance vs Cut value
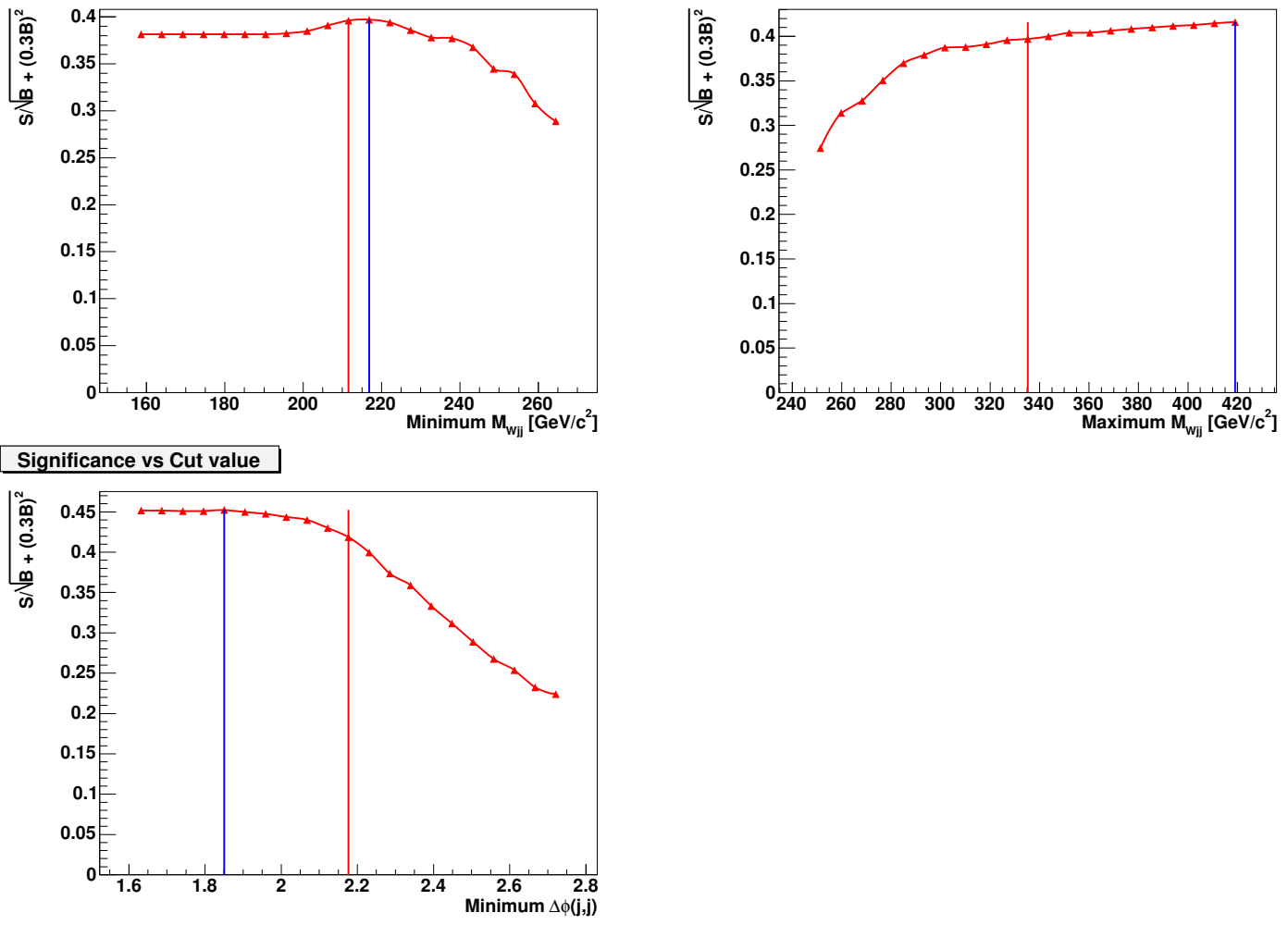

Figure A.20: Results of varying the selection cuts for $M_{\rho}=275 \mathrm{GeV} / c^{2}$, $M_{\pi}=150 \mathrm{GeV} / c^{2}$. The vertical red line indicates the postion of the initial grid search maximum. The blue line indicates the new maximum after examining the area around the initial maximum. 


\section{Appendix B}

\section{Summaries of Event Yields}

Presented here are tables describing how event yields vary with application of requirements on the dijet invariant mass $\left(m_{j j}\right)$, the mass of the $W$ plus dijet system $\left(m_{W j j}\right)$ and the azimuthal opening angle between the two jets $(\Delta \phi(j, j))$. The selection criteria vary with the assumed mass, as shown in Table 6.7 and Figure 6.11. Signal yields assume $M_{V}=100 \mathrm{GeV}$. 


\begin{tabular}{|l|lll|}
\hline sample & $m_{j j}$ & $m_{W j j}$ & $\Delta \phi(j, j)$ \\
\hline \hline$W \rightarrow \mu \nu$ & 0.86 & 0.39 & 0.030 \\
$W \rightarrow \tau \nu$ & 0.070 & 0.070 & 0.070 \\
$Z / \gamma^{*} \rightarrow \mu \mu(60-130)$ & 0.060 & 0.010 & 0 \\
$Z / \gamma^{*} \rightarrow \tau \tau(60-130)$ & 0.010 & 0 & \\
$Z / \gamma^{*} \rightarrow \mu \mu(60-130)$ & 0 & & \\
$Z / \gamma^{*} \rightarrow \tau \tau(60-130)$ & & & \\
$W j j \rightarrow \mu \nu j j$ & 8.4 & 2.1 & 1.4 \\
$Z j j \rightarrow \mu \mu j j$ & 0.79 & 0.16 & 0.11 \\
$W W \rightarrow l \nu j j$ & 1.6 & 0.50 & 0.34 \\
$W Z \rightarrow \mu \nu j j$ & 0.010 & 0 & 0 \\
$W Z \rightarrow j j l l$ & 0.040 & 0.010 & 0.010 \\
$W Z \rightarrow \mu \nu c \bar{c}$ & 0.080 & 0.020 & 0.010 \\
$W Z \rightarrow \mu \nu b \bar{b}$ & 0.36 & 0.090 & 0.050 \\
$Z Z \rightarrow \mu \mu j j$ & 0 & 0 & 0 \\
$Z Z \rightarrow \mu \mu c \bar{c}$ & 0.020 & 0.010 & 0 \\
$Z Z \rightarrow \mu \mu b \bar{b}$ & 0.100 & 0.020 & 0.010 \\
$t \bar{t} \rightarrow l \nu j j b \bar{b}$ & 0.44 & 0.070 & 0.040 \\
$t \bar{t} \rightarrow l \nu l \nu b \bar{b}$ & 0.89 & 0.100 & 0.040 \\
$q t b \rightarrow q \mu \nu b \bar{b}$ & 0.45 & 0.060 & 0.030 \\
$W^{*} \rightarrow t b \rightarrow \mu \nu b \bar{b}$ & 0.34 & 0.060 & 0.030 \\
$W b \bar{b} \rightarrow \mu \nu b \bar{b}$ & 2.2 & 0.65 & 0.42 \\
$Z b \bar{b} \rightarrow \mu \mu b \bar{b}$ & 0.30 & 0.070 & 0.040 \\
non-W/Z & 2.2 & 0.92 & -0.010 \\
\hline Total Background & 19 & 5.4 & 2.7 \\
\hline Data & 13 & 2 & 1 \\
\hline \hline$\rho_{T}^{ \pm} \rightarrow W \pm \pi_{T}^{0} \rightarrow \mu \nu b \bar{b}$ & 3.1 & 2.0 & 1.7 \\
$\rho_{T}^{0} \rightarrow W \pm \pi_{T}^{\mp} \rightarrow \mu \nu b \bar{c}$ & 2.2 & 1.2 & 1.0 \\
\hline Total Signal & 5.3 & 3.2 & 2.7 \\
\hline
\end{tabular}

Table B.1: Evolution of event yields with additional selection requirements at $M_{\rho}=175 \mathrm{GeV} / c^{2}, M_{\pi}=90 \mathrm{GeV} / c^{2}$, and $M_{V}=100 \mathrm{GeV}$. 


\begin{tabular}{|l|lll|}
\hline sample & $m_{j j}$ & $m_{W j j}$ & $\Delta \phi(j, j)$ \\
\hline \hline$W \rightarrow \mu \nu$ & 0.88 & 0.37 & 0.020 \\
$W \rightarrow \tau \nu$ & 0.070 & 0.070 & 0.070 \\
$Z / \gamma^{*} \rightarrow \mu \mu(60-130)$ & 0.070 & 0.010 & 0 \\
$Z / \gamma^{*} \rightarrow \tau \tau(60-130)$ & 0.010 & 0 & \\
$Z / \gamma^{*} \rightarrow \mu \mu(60-130)$ & 0 & & \\
$Z / \gamma^{*} \rightarrow \tau \tau(60-130)$ & & & \\
$W j j \rightarrow \mu \nu j j$ & 8.2 & 1.6 & 1.0 \\
$Z j j \rightarrow \mu \mu j j$ & 0.77 & 0.11 & 0.080 \\
$W W \rightarrow l \nu j j$ & 1.2 & 0.30 & 0.20 \\
$W Z \rightarrow \mu \nu j j$ & 0.010 & 0 & 0 \\
$W Z \rightarrow j j l l$ & 0.030 & 0.010 & 0.010 \\
$W Z \rightarrow \mu \nu c \bar{c}$ & 0.070 & 0.010 & 0.010 \\
$W Z \rightarrow \mu \nu b \bar{b}$ & 0.30 & 0.050 & 0.030 \\
$Z Z \rightarrow \mu \mu j j$ & 0 & 0 & 0 \\
$Z Z \rightarrow \mu \mu c \bar{c}$ & 0.020 & 0 & 0 \\
$Z Z \rightarrow \mu \mu b \bar{b}$ & 0.080 & 0.020 & 0.010 \\
$t \bar{t} \rightarrow l \nu j j b \bar{b}$ & 0.64 & 0.060 & 0.040 \\
$t \bar{t} \rightarrow l \nu l \nu b \bar{b}$ & 1.00 & 0.070 & 0.030 \\
$q t b \rightarrow q \mu \nu b \bar{b}$ & 0.51 & 0.050 & 0.030 \\
$W * \rightarrow t b \rightarrow \mu \nu b \bar{b}$ & 0.39 & 0.040 & 0.020 \\
$W b \bar{b} \rightarrow \mu \nu b \bar{b}$ & 2.0 & 0.46 & 0.32 \\
$Z b \bar{b} \rightarrow \mu \mu b \bar{b}$ & 0.30 & 0.050 & 0.030 \\
non-W/Z & 2.2 & 0.55 & -0.010 \\
\hline Total Background & 19 & 3.9 & 1.9 \\
\hline Data & 10 & 1 & 1 \\
\hline \hline$\rho_{T}^{ \pm} \rightarrow W \pi_{T}^{0} \rightarrow \mu \nu b \bar{b}$ & 1.0 & 0.51 & 0.42 \\
$\rho_{T}^{0} \rightarrow W \pi_{T}^{\mp} \rightarrow \mu \nu b \bar{c}$ & 0.82 & 0.31 & 0.25 \\
\hline Total Signal & 1.8 & 0.82 & 0.67 \\
\hline
\end{tabular}

Table B.2: Evolution of event yields with additional selection requirements at $M_{\rho}=180 \mathrm{GeV} / c^{2}, M_{\pi}=100 \mathrm{GeV} / c^{2}$, and $M_{V}=100 \mathrm{GeV}$. 


\begin{tabular}{|l|lll|}
\hline sample & $m_{j j}$ & $m_{W j j}$ & $\Delta \phi(j, j)$ \\
\hline \hline$W \rightarrow \mu \nu$ & 0.88 & 0.37 & 0.13 \\
$W \rightarrow \tau \nu$ & 0.070 & 0.070 & 0.070 \\
$Z / \gamma^{*} \rightarrow \mu \mu(60-130)$ & 0.090 & 0.010 & 0.010 \\
$Z / \gamma^{*} \rightarrow \tau \tau(60-130)$ & 0.010 & 0 & 0 \\
$Z / \gamma^{*} \rightarrow \mu \mu(60-130)$ & 0 & & \\
$Z / \gamma^{*} \rightarrow \tau \tau(60-130)$ & & & \\
$W j j \rightarrow \mu \nu j j$ & 8.2 & 1.8 & 1.4 \\
$Z j j \rightarrow \mu \mu j j$ & 0.78 & 0.13 & 0.090 \\
$W W \rightarrow l \nu j j$ & 1.4 & 0.36 & 0.30 \\
$W Z \rightarrow \mu \nu j j$ & 0.010 & 0 & 0 \\
$W Z \rightarrow j j l l$ & 0.030 & 0.010 & 0.010 \\
$W Z \rightarrow \mu \nu c \bar{c}$ & 0.070 & 0.010 & 0.010 \\
$W Z \rightarrow \mu \nu b \bar{b}$ & 0.32 & 0.060 & 0.050 \\
$Z Z \rightarrow \mu \mu j j$ & 0 & 0 & 0 \\
$Z Z \rightarrow \mu \mu c \bar{c}$ & 0.020 & 0 & 0 \\
$Z Z \rightarrow \mu \mu b \bar{b}$ & 0.090 & 0.020 & 0.020 \\
$t \bar{t} \rightarrow l \nu j j \bar{b}$ & 0.63 & 0.060 & 0.060 \\
$t \bar{t} \rightarrow l \nu l \nu b \bar{b}$ & 1.0 & 0.090 & 0.060 \\
$q t b \rightarrow q \mu \nu b \bar{b}$ & 0.52 & 0.060 & 0.040 \\
$W * \rightarrow t b \rightarrow \mu \nu b \bar{b}$ & 0.39 & 0.050 & 0.040 \\
$W b \bar{b} \rightarrow \mu \nu b \bar{b}$ & 2.0 & 0.54 & 0.46 \\
$Z b \bar{b} \rightarrow \mu \mu b \bar{b}$ & 0.32 & 0.050 & 0.050 \\
non-W/Z & 2.2 & 0.55 & 0.050 \\
\hline Total Background & 19 & 4.2 & 2.8 \\
\hline Data & 10 & 1 & 1 \\
\hline \hline$\rho_{T}^{ \pm} \rightarrow W \pi_{T}^{0} \rightarrow \mu \nu b \bar{b}$ & 3.3 & 1.5 & 1.4 \\
$\rho_{T}^{0} \rightarrow W \pi_{T}^{\mp} \rightarrow \mu \nu b \bar{c}$ & 2.5 & 1.0 & 1.00 \\
\hline Total Signal & 5.8 & 2.6 & 2.4 \\
\hline
\end{tabular}

Table B.3: Evolution of event yields with additional selection requirements at $M_{\rho}=190 \mathrm{GeV} / c^{2}, M_{\pi}=100 \mathrm{GeV} / c^{2}$, and $M_{V}=100 \mathrm{GeV}$. 


\begin{tabular}{|l|lll|}
\hline sample & $m_{j j}$ & $m_{W j j}$ & $\Delta \phi(j, j)$ \\
\hline \hline$W \rightarrow \mu \nu$ & 0.51 & 0.49 & 0.15 \\
$W \rightarrow \tau \nu$ & 0.070 & 0.070 & 0.070 \\
$Z / \gamma^{*} \rightarrow \mu \mu(60-130)$ & 0.080 & 0.060 & 0.010 \\
$Z / \gamma^{*} \rightarrow \tau \tau(60-130)$ & 0.010 & 0 & 0 \\
$Z / \gamma^{*} \rightarrow \mu \mu(60-130)$ & 0 & 0 & 0 \\
$Z / \gamma^{*} \rightarrow \tau \tau(60-130)$ & & & \\
$W j j \rightarrow \mu \nu j j$ & 8.0 & 4.2 & 2.1 \\
$Z j j \rightarrow \mu \mu j j$ & 0.70 & 0.36 & 0.18 \\
$W W \rightarrow l \nu j j$ & 0.70 & 0.43 & 0.21 \\
$W Z \rightarrow \mu \nu j j$ & 0.010 & 0 & 0 \\
$W Z \rightarrow j j l l$ & 0.020 & 0.010 & 0 \\
$W Z \rightarrow \mu \nu c \bar{c}$ & 0.050 & 0.020 & 0.010 \\
$W Z \rightarrow \mu \nu b \bar{b}$ & 0.21 & 0.100 & 0.050 \\
$Z Z \rightarrow \mu \mu j j$ & 0 & 0 & 0 \\
$Z Z \rightarrow \mu \mu c \bar{c}$ & 0.010 & 0.010 & 0 \\
$Z Z \rightarrow \mu \mu b \bar{b}$ & 0.060 & 0.030 & 0.010 \\
$t \bar{t} \rightarrow l \nu j j \bar{b}$ & 0.74 & 0.28 & 0.090 \\
$t \bar{t} \rightarrow l \nu l \nu b \bar{b}$ & 1.2 & 0.38 & 0.12 \\
$q t b \rightarrow q \mu \nu b \bar{b}$ & 0.55 & 0.28 & 0.12 \\
$W * \rightarrow t b \rightarrow \mu \nu b \bar{b}$ & 0.44 & 0.23 & 0.100 \\
$W b \bar{b} \rightarrow \mu \nu b \bar{b}$ & 1.7 & 0.92 & 0.60 \\
$Z b \bar{b} \rightarrow \mu \mu b \bar{b}$ & 0.28 & 0.13 & 0.060 \\
non-W/Z & 1.9 & 1.4 & 0.17 \\
\hline Total Background & 17 & 9.4 & 4.1 \\
\hline Data & 9 & 4 & 2 \\
\hline \hline$\rho_{T}^{ \pm} \rightarrow W \pi_{T}^{0} \rightarrow \mu \nu b \bar{b}$ & 0.70 & 0.59 & 0.45 \\
$\rho_{T}^{0} \rightarrow W \pi_{T}^{\mp} \rightarrow \mu \nu b \bar{c}$ & 0.76 & 0.54 & 0.38 \\
\hline Total Signal & 1.5 & 1.1 & 0.83 \\
\hline
\end{tabular}

Table B.4: Evolution of event yields with additional selection requirements at $M_{\rho}=190 \mathrm{GeV} / c^{2}, M_{\pi}=110 \mathrm{GeV} / c^{2}$, and $M_{V}=100 \mathrm{GeV}$. 


\begin{tabular}{|l|lll|}
\hline sample & $m_{j j}$ & $m_{W j j}$ & $\Delta \phi(j, j)$ \\
\hline \hline$W \rightarrow \mu \nu$ & 0.88 & 0.82 & 0.41 \\
$W \rightarrow \tau \nu$ & 0.070 & 0.070 & 0.070 \\
$Z / \gamma^{*} \rightarrow \mu \mu(60-130)$ & 0.080 & 0.050 & 0.020 \\
$Z / \gamma^{*} \rightarrow \tau \tau(60-130)$ & 0.010 & 0 & 0 \\
$Z / \gamma^{*} \rightarrow \mu \mu(60-130)$ & 0 & 0 & 0 \\
$Z / \gamma^{*} \rightarrow \tau \tau(60-130)$ & & & \\
$W j j \rightarrow \mu \nu j j$ & 8.2 & 5.2 & 3.5 \\
$Z j j \rightarrow \mu \mu j j$ & 0.78 & 0.48 & 0.29 \\
$W W \rightarrow l \nu j j$ & 1.3 & 0.91 & 0.59 \\
$W Z \rightarrow \mu \nu j j$ & 0.010 & 0.010 & 0 \\
$W Z \rightarrow j j l l$ & 0.030 & 0.020 & 0.010 \\
$W Z \rightarrow \mu \nu c \bar{c}$ & 0.070 & 0.040 & 0.030 \\
$W Z \rightarrow \mu \nu b \bar{b}$ & 0.32 & 0.18 & 0.12 \\
$Z Z \rightarrow \mu \mu j j$ & 0 & 0 & 0 \\
$Z Z \rightarrow \mu \mu c \bar{c}$ & 0.020 & 0.010 & 0.010 \\
$Z Z \rightarrow \mu \mu b \bar{b}$ & 0.090 & 0.050 & 0.040 \\
$t \bar{t} \rightarrow l \nu j j b \bar{b}$ & 0.63 & 0.29 & 0.18 \\
$t \bar{t} \rightarrow l \nu l \nu b \bar{b}$ & 1.0 & 0.42 & 0.20 \\
$q t b \rightarrow q \mu \nu b \bar{b}$ & 0.51 & 0.32 & 0.18 \\
$W^{*} \rightarrow t b \rightarrow \mu \nu b \bar{b}$ & 0.39 & 0.25 & 0.14 \\
$W b \bar{b} \rightarrow \mu \nu b \bar{b}$ & 2.0 & 1.3 & 1.1 \\
$Z b \bar{b} \rightarrow \mu \mu b \bar{b}$ & 0.31 & 0.17 & 0.11 \\
non-W/Z & 2.2 & 1.9 & 0.61 \\
\hline Total Background & 19 & 13 & 7.6 \\
\hline Data & 10 & 3 & 2 \\
\hline \hline$\rho_{T}^{ \pm} \rightarrow W \pi_{T}^{0} \rightarrow \mu \nu b \bar{b}$ & 2.6 & 2.2 & 1.9 \\
$\rho_{T}^{0} \rightarrow W \pi_{T}^{\mp} \rightarrow \mu \nu b \bar{c}$ & 2.8 & 2.1 & 1.8 \\
\hline Total Signal & 5.4 & 4.3 & 3.7 \\
\hline
\end{tabular}

Table B.5: Evolution of event yields with additional selection requirements at $M_{\rho}=195 \mathrm{GeV} / c^{2}, M_{\pi}=100 \mathrm{GeV} / c^{2}$, and $M_{V}=100 \mathrm{GeV}$. 


\begin{tabular}{|l|lll|}
\hline sample & $m_{j j}$ & $m_{W j j}$ & $\Delta \phi(j, j)$ \\
\hline \hline$W \rightarrow \mu \nu$ & 0.88 & 0.82 & 0.41 \\
$W \rightarrow \tau \nu$ & 0.070 & 0.070 & 0.070 \\
$Z / \gamma^{*} \rightarrow \mu \mu(60-130)$ & 0.090 & 0.060 & 0.010 \\
$Z / \gamma^{*} \rightarrow \tau \tau(60-130)$ & 0.010 & 0 & 0 \\
$Z / \gamma^{*} \rightarrow \mu \mu(60-130)$ & 0 & 0 & 0 \\
$Z / \gamma^{*} \rightarrow \tau \tau(60-130)$ & & & \\
$W j j \rightarrow \mu \nu j j$ & 8.4 & 5.2 & 3.2 \\
$Z j j \rightarrow \mu \mu j j$ & 0.78 & 0.48 & 0.29 \\
$W W \rightarrow l \nu j j$ & 1.4 & 0.88 & 0.55 \\
$W Z \rightarrow \mu \nu j j$ & 0.010 & 0 & 0 \\
$W Z \rightarrow j j l l$ & 0.030 & 0.020 & 0.010 \\
$W Z \rightarrow \mu \nu c \bar{c}$ & 0.070 & 0.040 & 0.030 \\
$W Z \rightarrow \mu \nu b \bar{b}$ & 0.32 & 0.18 & 0.12 \\
$Z Z \rightarrow \mu \mu j j$ & 0 & 0 & 0 \\
$Z Z \rightarrow \mu \mu c \bar{c}$ & 0.020 & 0.010 & 0.010 \\
$Z Z \rightarrow \mu \mu b \bar{b}$ & 0.090 & 0.050 & 0.040 \\
$t \bar{t} \rightarrow l \nu j j b \bar{b}$ & 0.64 & 0.29 & 0.18 \\
$t \bar{t} \rightarrow l \nu l \nu b \bar{b}$ & 1.0 & 0.41 & 0.19 \\
$q t b \rightarrow q \mu \nu b \bar{b}$ & 0.52 & 0.31 & 0.17 \\
$W^{*} \rightarrow t b \rightarrow \mu \nu b \bar{b}$ & 0.40 & 0.25 & 0.13 \\
$W b \bar{b} \rightarrow \mu \nu b \bar{b}$ & 2.0 & 1.3 & 1.0 \\
$Z b \bar{b} \rightarrow \mu \mu b \bar{b}$ & 0.32 & 0.17 & 0.11 \\
non-W/Z & 2.2 & 1.8 & 0.54 \\
\hline Total Background & 19 & 12 & 7.1 \\
\hline Data & 10 & 3 & 2 \\
\hline \hline$\rho_{T}^{ \pm} \rightarrow W \pm \pi_{T}^{0} \rightarrow \mu \nu b \bar{b}$ & 2.7 & 2.2 & 2.0 \\
$\rho_{T}^{0} \rightarrow W \pm \pi_{T}^{\mp} \rightarrow \mu \nu b \bar{c}$ & 2.7 & 2.1 & 1.8 \\
\hline Total Signal & 5.4 & 4.3 & 3.8 \\
\hline
\end{tabular}

Table B.6: Evolution of event yields with additional selection requirements at $M_{\rho}=200 \mathrm{GeV} / c^{2}, M_{\pi}=105 \mathrm{GeV} / c^{2}$, and $M_{V}=100 \mathrm{GeV}$. 


\begin{tabular}{|l|lll|}
\hline sample & $m_{j j}$ & $m_{W j j}$ & $\Delta \phi(j, j)$ \\
\hline \hline$W \rightarrow \mu \nu$ & 0.58 & 0.13 & 0.13 \\
$W \rightarrow \tau \nu$ & & & \\
$Z / \gamma^{*} \rightarrow \mu \mu(60-130)$ & 0.070 & 0.030 & 0 \\
$Z / \gamma^{*} \rightarrow \tau \tau(60-130)$ & 0.010 & 0 & \\
$Z / \gamma^{*} \rightarrow \mu \mu(60-130)$ & 0 & 0 & 0 \\
$Z / \gamma^{*} \rightarrow \tau \tau(60-130)$ & & & \\
$W j j \rightarrow \mu \nu j j$ & 5.5 & 1.3 & 0.92 \\
$Z j j \rightarrow \mu \mu j j$ & 0.40 & 0.090 & 0.080 \\
$W W \rightarrow l \nu j j$ & 0.17 & 0.050 & 0.030 \\
$W Z \rightarrow \mu \nu j j$ & 0 & 0 & 0 \\
$W Z \rightarrow j j l l$ & 0 & 0 & 0 \\
$W Z \rightarrow \mu \nu c \bar{c}$ & 0.020 & 0 & 0 \\
$W Z \rightarrow \mu \nu b \bar{b}$ & 0.080 & 0.020 & 0.010 \\
$Z Z \rightarrow \mu \mu j j$ & 0 & 0 & 0 \\
$Z Z \rightarrow \mu \mu c \bar{c}$ & 0 & 0 & 0 \\
$Z Z \rightarrow \mu \mu b \bar{b}$ & 0.020 & 0 & 0 \\
$t \bar{t} \rightarrow l \nu j j b \bar{b}$ & 0.67 & 0.090 & 0.040 \\
$t \bar{t} \rightarrow l \nu l \nu b \bar{b}$ & 1.0 & 0.12 & 0.050 \\
$q t b \rightarrow q \mu \nu b \bar{b}$ & 0.43 & 0.080 & 0.050 \\
$W^{*} \rightarrow t b \rightarrow \mu \nu b \bar{b}$ & 0.35 & 0.070 & 0.050 \\
$W b \bar{b} \rightarrow \mu \nu b \bar{b}$ & 1.2 & 0.24 & 0.19 \\
$Z b \bar{b} \rightarrow \mu \mu b \bar{b}$ & 0.19 & 0.040 & 0.020 \\
non-W/Z & 1.3 & 0.74 & 0.19 \\
\hline Total Background & 12 & 3.0 & 1.8 \\
\hline Data & 8 & 1 & 0 \\
\hline \hline$\rho_{T}^{ \pm} \rightarrow W \pi_{T}^{0} \rightarrow \mu \nu b \bar{b}$ & 0.49 & 0.27 & 0.23 \\
$\rho_{T}^{0} \rightarrow W \pi_{T}^{\mp} \rightarrow \mu \nu b \bar{c}$ & 0.52 & 0.21 & 0.18 \\
\hline Total Signal & 1.0 & 0.48 & 0.41 \\
\hline
\end{tabular}

Table B.7: Evolution of event yields with additional selection requirements at $M_{\rho}=200 \mathrm{GeV} / c^{2}, M_{\pi}=120 \mathrm{GeV} / c^{2}$, and $M_{V}=100 \mathrm{GeV}$. 


\begin{tabular}{|l|lll|}
\hline sample & $m_{j j}$ & $m_{W j j}$ & $\Delta \phi(j, j)$ \\
\hline \hline$W \rightarrow \mu \nu$ & 0.30 & 0.28 & 0.27 \\
$W \rightarrow \tau \nu$ & & & \\
$Z / \gamma^{*} \rightarrow \mu \mu(60-130)$ & 0.060 & 0.040 & 0.010 \\
$Z / \gamma^{*} \rightarrow \tau \tau(60-130)$ & 0.010 & 0 & 0 \\
$Z / \gamma^{*} \rightarrow \mu \mu(60-130)$ & 0 & 0 & 0 \\
$Z / \gamma^{*} \rightarrow \tau \tau(60-130)$ & & & \\
$W j j \rightarrow \mu \nu j j$ & 5.6 & 3.5 & 2.8 \\
$Z j j \rightarrow \mu \mu j j$ & 0.44 & 0.33 & 0.23 \\
$W W \rightarrow l \nu j j$ & 0.30 & 0.21 & 0.16 \\
$W Z \rightarrow \mu \nu j j$ & 0 & 0 & 0 \\
$W Z \rightarrow j j l l$ & 0.010 & 0.010 & 0.010 \\
$W Z \rightarrow \mu \nu c \bar{c}$ & 0.030 & 0.020 & 0.010 \\
$W Z \rightarrow \mu \nu b \bar{b}$ & 0.11 & 0.080 & 0.060 \\
$Z Z \rightarrow \mu \mu j j$ & 0 & 0 & 0 \\
$Z Z \rightarrow \mu \mu c \bar{c}$ & 0.010 & 0 & 0 \\
$Z Z \rightarrow \mu \mu b \bar{b}$ & 0.030 & 0.020 & 0.020 \\
$t \bar{t} \rightarrow l \nu j j b \bar{b}$ & 0.58 & 0.37 & 0.21 \\
$t \bar{t} \rightarrow l \nu l \nu b \bar{b}$ & 0.83 & 0.47 & 0.32 \\
$q t b \rightarrow q \mu \nu b \bar{b}$ & 0.40 & 0.32 & 0.22 \\
$W^{*} \rightarrow t b \rightarrow \mu \nu b \bar{b}$ & 0.30 & 0.23 & 0.16 \\
$W b \bar{b} \rightarrow \mu \nu b \bar{b}$ & 1.1 & 0.77 & 0.70 \\
$Z b \bar{b} \rightarrow \mu \mu b \bar{b}$ & 0.19 & 0.12 & 0.100 \\
non-W/Z & 1.5 & 1.3 & 0.59 \\
\hline Total Background & 12 & 8.1 & 5.9 \\
\hline Data & 5 & 4 & 4 \\
\hline \hline$\rho_{T}^{ \pm} \rightarrow W \pm \pi_{T}^{0} \rightarrow \mu \nu b \bar{b}$ & 1.5 & 1.3 & 1.2 \\
$\rho_{T}^{0} \rightarrow W \pi_{T}^{\mp} \rightarrow \mu \nu b \bar{c}$ & 1.8 & 1.5 & 1.4 \\
\hline Total Signal & 3.3 & 2.9 & 2.6 \\
\hline
\end{tabular}

Table B.8: Evolution of event yields with additional selection requirements at $M_{\rho}=210 \mathrm{GeV} / c^{2}, M_{\pi}=110 \mathrm{GeV} / c^{2}$, and $M_{V}=100 \mathrm{GeV}$. 


\begin{tabular}{|c|c|c|c|}
\hline sample & $m_{j j}$ & $m_{W j j}$ & $\Delta \phi(j, j)$ \\
\hline $\bar{~} W \rightarrow \mu \nu$ & 0.97 & 0.22 & 0.13 \\
\hline$W \rightarrow \tau \nu$ & & & \\
\hline$Z / \gamma^{*} \rightarrow \mu \mu(60-130)$ & 0.080 & 0.050 & 0.020 \\
\hline$Z / \gamma^{*} \rightarrow \tau \tau(60-130)$ & 0.010 & 0 & 0 \\
\hline$Z / \gamma^{*} \rightarrow \mu \mu(60-130)$ & 0 & 0 & 0 \\
\hline \multicolumn{4}{|l|}{$Z / \gamma^{*} \rightarrow \tau \tau(60-130)$} \\
\hline$W j j \rightarrow \mu \nu j j$ & 7.2 & 2.6 & 1.4 \\
\hline$Z j j \rightarrow \mu \mu j j$ & 0.56 & 0.20 & 0.13 \\
\hline$W W \rightarrow l \nu j j$ & 0.24 & 0.14 & 0.070 \\
\hline$W Z \rightarrow \mu \nu j j$ & 0 & 0 & 0 \\
\hline$W Z \rightarrow j j l l$ & 0.010 & 0 & 0 \\
\hline$W Z \rightarrow \mu \nu c \bar{c}$ & 0.020 & 0.010 & 0 \\
\hline$W Z \rightarrow \mu \nu b \bar{b}$ & 0.100 & 0.040 & 0.020 \\
\hline$Z Z \rightarrow \mu \mu j j$ & 0 & 0 & 0 \\
\hline$Z Z \rightarrow \mu \mu c \bar{c}$ & 0.010 & 0 & 0 \\
\hline$Z Z \rightarrow \mu \mu b \bar{b}$ & 0.030 & 0.010 & 0.010 \\
\hline$t \bar{t} \rightarrow l \nu j j b \bar{b}$ & 1.1 & 0.27 & 0.100 \\
\hline$t \bar{t} \rightarrow l \nu l \nu b \bar{b}$ & 1.5 & 0.32 & 0.12 \\
\hline$q t b \rightarrow q \mu \nu b \bar{b}$ & 0.65 & 0.22 & 0.11 \\
\hline$W^{*} \rightarrow t b \rightarrow \mu \nu b \bar{b}$ & 0.50 & 0.17 & 0.090 \\
\hline$W b \bar{b} \rightarrow \mu \nu b \bar{b}$ & 1.5 & 0.61 & 0.42 \\
\hline$Z b \bar{b} \rightarrow \mu \mu b \bar{b}$ & 0.25 & 0.080 & 0.040 \\
\hline non- $W / Z$ & 1.9 & 1.1 & 0.25 \\
\hline Total Background & 17 & 6.0 & 3.0 \\
\hline Data & 12 & 2 & 0 \\
\hline$\rho_{T}^{ \pm} \rightarrow W^{ \pm} \pi_{T}^{0} \rightarrow \mu \nu b \bar{b}$ & 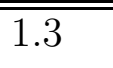 & 0.87 & 0.77 \\
\hline$\rho_{T}^{0} \rightarrow W^{ \pm} \pi_{T}^{\mp} \rightarrow \mu \nu b \bar{c}$ & 1.2 & 0.68 & 0.56 \\
\hline Total Signal & 2.5 & 1.6 & 1.3 \\
\hline
\end{tabular}

Table B.9: Evolution of event yields with additional selection requirements at $M_{\rho}=215 \mathrm{GeV} / c^{2}, M_{\pi}=130 \mathrm{GeV} / c^{2}$, and $M_{V}=100 \mathrm{GeV}$. 


\begin{tabular}{|c|c|c|c|}
\hline sample & $m_{j j}$ & $m_{W j j}$ & $\Delta \phi(j, j)$ \\
\hline$W \rightarrow \mu \nu$ & 0.59 & 0.49 & 0.48 \\
\hline$W \rightarrow \tau \nu$ & & & \\
\hline$Z / \gamma^{*} \rightarrow \mu \mu(60-130)$ & 0.070 & 0.050 & 0.020 \\
\hline$Z / \gamma^{*} \rightarrow \tau \tau(60-130)$ & 0.010 & 0 & 0 \\
\hline$Z / \gamma^{*} \rightarrow \mu \mu(60-130)$ & 0 & 0 & 0 \\
\hline$Z / \gamma^{*} \rightarrow \tau \tau(60-130)$ & & & \\
\hline$W j j \rightarrow \mu \nu j j$ & 5.7 & 3.0 & 2.4 \\
\hline$Z j j \rightarrow \mu \mu j j$ & 0.42 & 0.23 & 0.18 \\
\hline$W W \rightarrow l \nu j j$ & 0.17 & 0.11 & 0.090 \\
\hline$W Z \rightarrow \mu \nu j j$ & 0 & 0 & 0 \\
\hline$W Z \rightarrow j j l l$ & 0.010 & 0 & 0 \\
\hline$W Z \rightarrow \mu \nu c \bar{c}$ & 0.020 & 0.010 & 0.010 \\
\hline$W Z \rightarrow \mu \nu b \bar{b}$ & 0.080 & 0.040 & 0.040 \\
\hline$Z Z \rightarrow \mu \mu j j$ & 0 & 0 & 0 \\
\hline$Z Z \rightarrow \mu \mu c \bar{c}$ & 0 & 0 & 0 \\
\hline$Z Z \rightarrow \mu \mu b \bar{b}$ & 0.020 & 0.010 & 0.010 \\
\hline$t \bar{t} \rightarrow l \nu j j b \bar{b}$ & 0.71 & 0.37 & 0.22 \\
\hline$t \bar{t} \rightarrow l \nu l \nu b \bar{b}$ & 1.1 & 0.45 & 0.34 \\
\hline$q t b \rightarrow q \mu \nu b \bar{b}$ & 0.47 & 0.28 & 0.21 \\
\hline$W^{*} \rightarrow t b \rightarrow \mu \nu b \bar{b}$ & 0.38 & 0.22 & 0.16 \\
\hline$W b \bar{b} \rightarrow \mu \nu b \bar{b}$ & 1.2 & 0.68 & 0.62 \\
\hline$Z b \bar{b} \rightarrow \mu \mu b \bar{b}$ & 0.20 & 0.11 & 0.100 \\
\hline non- $W / Z$ & 1.4 & 1.5 & 0.65 \\
\hline Total Background & 13 & 7.5 & 5.6 \\
\hline Data & 9 & 5 & 5 \\
\hline$\overline{\rho_{T}^{ \pm}} \rightarrow W^{ \pm} \pi_{T}^{0} \rightarrow \mu \nu b \bar{b}$ & 1.8 & 1.3 & 1.2 \\
\hline$\rho_{T}^{0} \rightarrow W^{ \pm} \pi_{T}^{\mp} \rightarrow \mu \nu b \bar{c}$ & 1.7 & 1.2 & 1.1 \\
\hline Total Signal & 3.5 & 2.5 & 2.4 \\
\hline
\end{tabular}

Table B.10: Evolution of event yields with additional selection requirements at $M_{\rho}=220 \mathrm{GeV} / c^{2}, M_{\pi}=120 \mathrm{GeV} / c^{2}$, and $M_{V}=100 \mathrm{GeV}$. 


\begin{tabular}{|c|c|c|c|}
\hline sample & $m_{j j}$ & $m_{W j j}$ & $\Delta \phi(j, j)$ \\
\hline $\bar{~} W \rightarrow \mu \nu$ & 0.96 & 0.55 & $\overline{0.19}$ \\
\hline$W \rightarrow \tau \nu$ & & & \\
\hline$Z / \gamma^{*} \rightarrow \mu \mu(60-130)$ & 0.030 & 0.020 & 0.010 \\
\hline$Z / \gamma^{*} \rightarrow \tau \tau(60-130)$ & 0 & 0 & 0 \\
\hline$Z / \gamma^{*} \rightarrow \mu \mu(60-130)$ & 0 & 0 & 0 \\
\hline \multicolumn{4}{|l|}{$Z / \gamma^{*} \rightarrow \tau \tau(60-130)$} \\
\hline$W j j \rightarrow \mu \nu j j$ & 5.8 & 2.9 & 1.7 \\
\hline$Z j j \rightarrow \mu \mu j j$ & 0.39 & 0.21 & 0.15 \\
\hline$W W \rightarrow l \nu j j$ & 0.050 & 0.030 & 0.020 \\
\hline$W Z \rightarrow \mu \nu j j$ & 0 & 0 & 0 \\
\hline$W Z \rightarrow j j l l$ & 0 & 0 & 0 \\
\hline$W Z \rightarrow \mu \nu c \bar{c}$ & 0.010 & 0 & 0 \\
\hline$W Z \rightarrow \mu \nu b \bar{b}$ & 0.040 & 0.020 & 0.010 \\
\hline$Z Z \rightarrow \mu \mu j j$ & 0 & 0 & 0 \\
\hline$Z Z \rightarrow \mu \mu c \bar{c}$ & 0 & 0 & 0 \\
\hline$Z Z \rightarrow \mu \mu b \bar{b}$ & 0.010 & 0 & 0 \\
\hline$t \bar{t} \rightarrow l \nu j j b \bar{b}$ & 1.1 & 0.44 & 0.20 \\
\hline$t \bar{t} \rightarrow l \nu l \nu b \bar{b}$ & 1.4 & 0.48 & 0.26 \\
\hline$q t b \rightarrow q \mu \nu b \bar{b}$ & 0.60 & 0.31 & 0.15 \\
\hline$W^{*} \rightarrow t b \rightarrow \mu \nu b \bar{b}$ & 0.46 & 0.24 & 0.13 \\
\hline$W b \bar{b} \rightarrow \mu \nu b \bar{b}$ & 1.1 & 0.55 & 0.37 \\
\hline$Z b \bar{b} \rightarrow \mu \mu b \bar{b}$ & 0.19 & 0.100 & 0.060 \\
\hline non- $W / Z$ & 1.4 & 1.1 & 0.22 \\
\hline Total Background & 14 & 7.0 & 3.5 \\
\hline Data & 13 & 7 & 4 \\
\hline 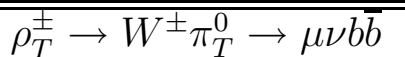 & 0.36 & 0.29 & 0.25 \\
\hline$\rho_{T}^{0} \rightarrow W^{ \pm} \pi_{T}^{\mp} \rightarrow \mu \nu b \bar{c}$ & 0.41 & 0.29 & 0.23 \\
\hline Total Signal & 0.77 & 0.57 & 0.48 \\
\hline
\end{tabular}

Table B.11: Evolution of event yields with additional selection requirements at $M_{\rho}=220 \mathrm{GeV} / c^{2}, M_{\pi}=140 \mathrm{GeV} / c^{2}$, and $M_{V}=100 \mathrm{GeV}$. 


\begin{tabular}{|l|lll|}
\hline sample & $m_{j j}$ & $m_{W j j}$ & $\Delta \phi(j, j)$ \\
\hline \hline$W \rightarrow \mu \nu$ & 0.67 & 0.64 & 0.63 \\
$W \rightarrow \tau \nu$ & 0.070 & & \\
$Z / \gamma^{*} \rightarrow \mu \mu(60-130)$ & 0.080 & 0.070 & 0.040 \\
$Z / \gamma^{*} \rightarrow \tau \tau(60-130)$ & 0.010 & 0.010 & 0 \\
$Z / \gamma^{*} \rightarrow \mu \mu(60-130)$ & 0 & & \\
$Z / \gamma^{*} \rightarrow \tau \tau(60-130)$ & & & \\
$W j j \rightarrow \mu \nu j j$ & 7.1 & 5.7 & 4.9 \\
$Z j j \rightarrow \mu \mu j j$ & 0.60 & 0.55 & 0.46 \\
$W W \rightarrow l \nu j j$ & 0.44 & 0.33 & 0.25 \\
$W Z \rightarrow \mu \nu j j$ & 0.010 & 0 & 0 \\
$W Z \rightarrow j j l l$ & 0.010 & 0.010 & 0.010 \\
$W Z \rightarrow \mu \nu c \bar{c}$ & 0.030 & 0.030 & 0.020 \\
$W Z \rightarrow \mu \nu b \bar{b}$ & 0.15 & 0.12 & 0.100 \\
$Z Z \rightarrow \mu \mu j j$ & 0 & 0 & 0 \\
$Z Z \rightarrow \mu \mu c \bar{c}$ & 0.010 & 0.010 & 0.010 \\
$Z Z \rightarrow \mu \mu b \bar{b}$ & 0.040 & 0.030 & 0.030 \\
$t \bar{t} \rightarrow l \nu j j b \bar{b}$ & 0.73 & 0.65 & 0.52 \\
$t \bar{t} \rightarrow l \nu l \nu b \bar{b}$ & 1.1 & 0.96 & 0.77 \\
$q t b \rightarrow q \mu \nu b \bar{b}$ & 0.50 & 0.44 & 0.34 \\
$W^{*} \rightarrow t b \rightarrow \mu \nu b \bar{b}$ & 0.41 & 0.36 & 0.29 \\
$W b \bar{b} \rightarrow \mu \nu b \bar{b}$ & 1.4 & 1.2 & 1.1 \\
$Z b \bar{b} \rightarrow \mu \mu b \bar{b}$ & 0.25 & 0.21 & 0.18 \\
non-W/Z & 1.7 & 1.3 & 0.88 \\
\hline Total Background & 15 & 13 & 11 \\
\hline Data & 8 & 8 & 7 \\
\hline \hline$\rho_{T}^{ \pm} \rightarrow W \pi_{T}^{0} \rightarrow \mu \nu b \bar{b}$ & 1.4 & 1.3 & 1.2 \\
$\rho_{T}^{0} \rightarrow W \pm \pi_{T}^{\mp} \rightarrow \mu \nu b \bar{c}$ & 1.7 & 1.6 & 1.5 \\
\hline Total Signal & 3.1 & 2.9 & 2.6 \\
\hline
\end{tabular}

Table B.12: Evolution of event yields with additional selection requirements at $M_{\rho}=235 \mathrm{GeV} / c^{2}, M_{\pi}=120 \mathrm{GeV} / c^{2}$, and $M_{V}=100 \mathrm{GeV}$. 


\begin{tabular}{|l|lll|}
\hline sample & $m_{j j}$ & $m_{W j j}$ & $\Delta \phi(j, j)$ \\
\hline \hline$W \rightarrow \mu \nu$ & 0.95 & 0.55 & 0.54 \\
$W \rightarrow \tau \nu$ & & & \\
$Z / \gamma^{*} \rightarrow \mu \mu(60-130)$ & 0.060 & 0.050 & 0.020 \\
$Z / \gamma^{*} \rightarrow \tau \tau(60-130)$ & 0.010 & 0 & 0 \\
$Z / \gamma^{*} \rightarrow \mu \mu(60-130)$ & 0 & 0 & 0 \\
$Z / \gamma^{*} \rightarrow \tau \tau(60-130)$ & & & \\
$W j j \rightarrow \mu \nu j j$ & 5.3 & 3.0 & 2.7 \\
$Z j j \rightarrow \mu \mu j j$ & 0.41 & 0.26 & 0.23 \\
$W W \rightarrow l \nu j j$ & 0.100 & 0.060 & 0.060 \\
$W Z \rightarrow \mu \nu j j$ & 0 & 0 & 0 \\
$W Z \rightarrow j j l l$ & 0 & 0 & 0 \\
$W Z \rightarrow \mu \nu c \bar{c}$ & 0.010 & 0.010 & 0.010 \\
$W Z \rightarrow \mu \nu b \bar{b}$ & 0.040 & 0.030 & 0.020 \\
$Z Z \rightarrow \mu \mu j j$ & 0 & 0 & 0 \\
$Z Z \rightarrow \mu \mu c \bar{c}$ & 0 & 0 & 0 \\
$Z Z \rightarrow \mu \mu b \bar{b}$ & 0.010 & 0.010 & 0.010 \\
$t \bar{t} \rightarrow l \nu j j b \bar{b}$ & 0.93 & 0.53 & 0.42 \\
$t \bar{t} \rightarrow l \nu l \nu b \bar{b}$ & 1.2 & 0.54 & 0.47 \\
$q t b \rightarrow q \mu \nu b \bar{b}$ & 0.52 & 0.34 & 0.27 \\
$W^{*} \rightarrow t b \rightarrow \mu \nu b \bar{b}$ & 0.41 & 0.27 & 0.23 \\
$W b \bar{b} \rightarrow \mu \nu b \bar{b}$ & 1.1 & 0.66 & 0.61 \\
$Z b \bar{b} \rightarrow \mu \mu b \bar{b}$ & 0.19 & 0.11 & 0.100 \\
non-W/Z & 1.1 & 1.1 & 0.56 \\
\hline Total Background & 12 & 7.5 & 6.2 \\
\hline Data & 12 & 8 & 8 \\
\hline \hline$\rho_{T}^{ \pm} \rightarrow W \pm \pi_{T}^{0} \rightarrow \mu \nu b \bar{b}$ & 1.4 & 0.97 & 0.92 \\
$\rho_{T}^{0} \rightarrow W \pm \pi_{T}^{\mp} \rightarrow \mu \nu b \bar{c}$ & 1.6 & 1.1 & 1.1 \\
\hline Total Signal & 3.0 & 2.1 & 2.0 \\
\hline
\end{tabular}

Table B.13: Evolution of event yields with additional selection requirements at $M_{\rho}=235 \mathrm{GeV} / c^{2}, M_{\pi}=130 \mathrm{GeV} / c^{2}$, and $M_{V}=100 \mathrm{GeV}$. 


\begin{tabular}{|l|lll|}
\hline sample & $m_{j j}$ & $m_{W j j}$ & $\Delta \phi(j, j)$ \\
\hline \hline$W \rightarrow \mu \nu$ & 0.75 & 0.35 & 0.070 \\
$W \rightarrow \tau \nu$ & & & \\
$Z / \gamma^{*} \rightarrow \mu \mu(60-130)$ & 0.020 & 0.020 & 0.020 \\
$Z / \gamma^{*} \rightarrow \tau \tau(60-130)$ & 0 & 0 & 0 \\
$Z / \gamma^{*} \rightarrow \mu \mu(60-130)$ & 0 & 0 & 0 \\
$Z / \gamma^{*} \rightarrow \tau \tau(60-130)$ & & & \\
$W j j \rightarrow \mu \nu j j$ & 3.3 & 2.2 & 1.2 \\
$Z j j \rightarrow \mu \mu j j$ & 0.22 & 0.12 & 0.060 \\
$W W \rightarrow l \nu j j$ & 0.020 & 0.010 & 0.010 \\
$W Z \rightarrow \mu \nu j j$ & 0 & 0 & 0 \\
$W Z \rightarrow j j l l$ & 0 & 0 & 0 \\
$W Z \rightarrow \mu \nu c \bar{c}$ & 0 & 0 & 0 \\
$W Z \rightarrow \mu \nu b \bar{b}$ & 0.010 & 0.010 & 0 \\
$Z Z \rightarrow \mu \mu j j$ & 0 & 0 & 0 \\
$Z Z \rightarrow \mu \mu c \bar{c}$ & 0 & 0 & 0 \\
$Z Z \rightarrow \mu \mu b \bar{b}$ & 0 & 0 & 0 \\
$t \bar{t} \rightarrow l \nu j j \bar{b}$ & 0.84 & 0.49 & 0.20 \\
$t \bar{t} \rightarrow l \nu l \nu b \bar{b}$ & 1.1 & 0.51 & 0.22 \\
$q t b \rightarrow q \mu \nu b \bar{b}$ & 0.44 & 0.29 & 0.12 \\
$W * \rightarrow t b \rightarrow \mu \nu b \bar{b}$ & 0.32 & 0.22 & 0.11 \\
$W b \bar{b} \rightarrow \mu \nu b \bar{b}$ & 0.70 & 0.40 & 0.26 \\
$Z b \bar{b} \rightarrow \mu \mu b \bar{b}$ & 0.12 & 0.080 & 0.050 \\
non-W/Z & 1.0 & 0.76 & 0.100 \\
\hline Total Background & 8.9 & 5.5 & 2.5 \\
\hline Data & 10 & 6 & 3 \\
\hline \hline$\rho_{T}^{ \pm} \rightarrow W \pi_{T}^{0} \rightarrow \mu \nu b \bar{b}$ & 0.65 & 0.56 & 0.48 \\
$\rho_{T}^{0} \rightarrow W \pi_{T}^{\mp} \rightarrow \mu \nu b \bar{c}$ & 0.69 & 0.59 & 0.48 \\
\hline Total Signal & 1.3 & 1.1 & 0.96 \\
\hline
\end{tabular}

Table B.14: Evolution of event yields with additional selection requirements at $M_{\rho}=235 \mathrm{GeV} / c^{2}, M_{\pi}=150 \mathrm{GeV} / c^{2}$, and $M_{V}=100 \mathrm{GeV}$. 


\begin{tabular}{|l|lll|}
\hline sample & $m_{j j}$ & $m_{W j j}$ & $\Delta \phi(j, j)$ \\
\hline \hline$W \rightarrow \mu \nu$ & 0.87 & 0.46 & 0.46 \\
$W \rightarrow \tau \nu$ & & & \\
$Z / \gamma^{*} \rightarrow \mu \mu(60-130)$ & 0.030 & 0.020 & 0.020 \\
$Z / \gamma^{*} \rightarrow \tau \tau(60-130)$ & 0 & 0 & 0 \\
$Z / \gamma^{*} \rightarrow \mu \mu(60-130)$ & 0 & 0 & 0 \\
$Z / \gamma^{*} \rightarrow \tau \tau(60-130)$ & & & \\
$W j j \rightarrow \mu \nu j j$ & 4.0 & 2.7 & 2.3 \\
$Z j j \rightarrow \mu \mu j j$ & 0.28 & 0.19 & 0.17 \\
$W W \rightarrow l \nu j j$ & 0.030 & 0.030 & 0.020 \\
$W Z \rightarrow \mu \nu j j$ & 0 & 0 & 0 \\
$W Z \rightarrow j j l l$ & 0 & 0 & 0 \\
$W Z \rightarrow \mu \nu c \bar{c}$ & 0.010 & 0 & 0 \\
$W Z \rightarrow \mu \nu b \bar{b}$ & 0.020 & 0.020 & 0.010 \\
$Z Z \rightarrow \mu \mu j j$ & 0 & 0 & 0 \\
$Z Z \rightarrow \mu \mu c \bar{c}$ & 0 & 0 & 0 \\
$Z Z \rightarrow \mu \mu b \bar{b}$ & 0.010 & 0 & 0 \\
$t \bar{t} \rightarrow l \nu j j b \bar{b}$ & 0.62 & 0.41 & 0.31 \\
$t \bar{t} \rightarrow l \nu l \nu b \bar{b}$ & 0.87 & 0.46 & 0.35 \\
$q t b \rightarrow q \mu \nu b \bar{b}$ & 0.37 & 0.26 & 0.20 \\
$W * \rightarrow t b \rightarrow \mu \nu b \bar{b}$ & 0.29 & 0.22 & 0.17 \\
$W b \bar{b} \rightarrow \mu \nu b \bar{b}$ & 0.73 & 0.47 & 0.41 \\
$Z b \bar{b} \rightarrow \mu \mu b \bar{b}$ & 0.12 & 0.090 & 0.070 \\
non-W/Z & 0.79 & 0.67 & 0.14 \\
\hline Total Background & 9.1 & 6.0 & 4.6 \\
\hline Data & 11 & 10 & 6 \\
\hline \hline$\rho_{T}^{ \pm} \rightarrow W \pi_{T}^{0} \rightarrow \mu \nu b \bar{b}$ & 0.99 & 0.83 & 0.76 \\
$\rho_{T}^{0} \rightarrow W \pi_{T}^{\mp} \rightarrow \mu \nu b \bar{c}$ & 1.2 & 1.00 & 0.90 \\
\hline Total Signal & 2.2 & 1.8 & 1.7 \\
\hline
\end{tabular}

Table B.15: Evolution of event yields with additional selection requirements at $M_{\rho}=240 \mathrm{GeV} / c^{2}, M_{\pi}=140 \mathrm{GeV} / c^{2}$, and $M_{V}=100 \mathrm{GeV}$. 


\begin{tabular}{|l|lll|}
\hline sample & $m_{j j}$ & $m_{W j j}$ & $\Delta \phi(j, j)$ \\
\hline \hline$W \rightarrow \mu \nu$ & 0.91 & 0.90 & 0.89 \\
$W \rightarrow \tau \nu$ & & & \\
$Z / \gamma^{*} \rightarrow \mu \mu(60-130)$ & 0.060 & 0.030 & 0.020 \\
$Z / \gamma^{*} \rightarrow \tau \tau(60-130)$ & 0.010 & 0.010 & 0.010 \\
$Z / \gamma^{*} \rightarrow \mu \mu(60-130)$ & & & \\
$Z / \gamma^{*} \rightarrow \tau \tau(60-130)$ & & & \\
$W j j \rightarrow \mu \nu j j$ & 4.9 & 4.5 & 3.9 \\
$Z j j \rightarrow \mu \mu j j$ & 0.37 & 0.30 & 0.28 \\
$W W \rightarrow l \nu j j$ & 0.090 & 0.080 & 0.060 \\
$W Z \rightarrow \mu \nu j j$ & 0 & 0 & 0 \\
$W Z \rightarrow j j l l$ & 0 & 0 & 0 \\
$W Z \rightarrow \mu \nu c \bar{c}$ & 0.010 & 0.010 & 0.010 \\
$W Z \rightarrow \mu \nu b \bar{b}$ & 0.040 & 0.030 & 0.030 \\
$Z Z \rightarrow \mu \mu j j$ & 0 & 0 & 0 \\
$Z Z \rightarrow \mu \mu c \bar{c}$ & 0 & 0 & 0 \\
$Z Z \rightarrow \mu \mu b \bar{b}$ & 0.010 & 0.010 & 0.010 \\
$t \bar{t} \rightarrow l \nu j j b \bar{b}$ & 0.79 & 0.72 & 0.67 \\
$t \bar{t} \rightarrow l \nu l \nu b \bar{b}$ & 1.0 & 0.87 & 0.78 \\
$q t b \rightarrow q \mu \nu b \bar{b}$ & 0.45 & 0.41 & 0.35 \\
$W^{*} \rightarrow t b \rightarrow \mu \nu b \bar{b}$ & 0.36 & 0.34 & 0.30 \\
$W b \bar{b} \rightarrow \mu \nu b \bar{b}$ & 0.98 & 0.86 & 0.81 \\
$Z b \bar{b} \rightarrow \mu \mu b \bar{b}$ & 0.17 & 0.14 & 0.13 \\
non-W/Z & 1.0 & 0.85 & 0.54 \\
\hline Total Background & 11 & 10 & 8.8 \\
\hline Data & 11 & 11 & 10 \\
\hline \hline$\rho_{T}^{ \pm} \rightarrow W \pi_{T}^{0} \rightarrow \mu \nu b \bar{b}$ & 0.94 & 0.88 & 0.82 \\
$\rho_{T}^{0} \rightarrow W \pi_{T}^{\mp} \rightarrow \mu \nu b \bar{c}$ & 1.3 & 1.2 & 1.1 \\
\hline Total Signal & 2.2 & 2.1 & 1.9 \\
\hline
\end{tabular}

Table B.16: Evolution of event yields with additional selection requirements at $M_{\rho}=250 \mathrm{GeV} / c^{2}, M_{\pi}=130 \mathrm{GeV} / c^{2}$, and $M_{V}=100 \mathrm{GeV}$. 


\begin{tabular}{|c|c|c|c|}
\hline sample & $m_{j j}$ & $m_{W j j}$ & $\Delta \phi(j, j)$ \\
\hline $\bar{~} W \rightarrow \mu \nu$ & 0.96 & 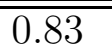 & 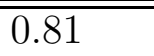 \\
\hline$W \rightarrow \tau \nu$ & & & \\
\hline$Z / \gamma^{*} \rightarrow \mu \mu(60-130)$ & 0.060 & 0.030 & 0.030 \\
\hline$Z / \gamma^{*} \rightarrow \tau \tau(60-130)$ & 0.010 & 0.010 & 0.010 \\
\hline$Z / \gamma^{*} \rightarrow \mu \mu(60-130)$ & 0 & 0 & 0 \\
\hline \multicolumn{4}{|l|}{$Z / \gamma^{*} \rightarrow \tau \tau(60-130)$} \\
\hline$W j j \rightarrow \mu \nu j j$ & 6.1 & 5.1 & 4.4 \\
\hline$Z j j \rightarrow \mu \mu j j$ & 0.48 & 0.40 & 0.35 \\
\hline$W W \rightarrow l \nu j j$ & 0.14 & 0.11 & 0.090 \\
\hline$W Z \rightarrow \mu \nu j j$ & 0 & 0 & 0 \\
\hline$W Z \rightarrow j j l l$ & 0 & 0 & 0 \\
\hline$W Z \rightarrow \mu \nu c \bar{c}$ & 0.010 & 0.010 & 0.010 \\
\hline$W Z \rightarrow \mu \nu b \bar{b}$ & 0.060 & 0.050 & 0.040 \\
\hline$Z Z \rightarrow \mu \mu j j$ & 0 & 0 & 0 \\
\hline$Z Z \rightarrow \mu \mu c \bar{c}$ & 0 & 0 & 0 \\
\hline$Z Z \rightarrow \mu \mu b \bar{b}$ & 0.020 & 0.010 & 0.010 \\
\hline$t \bar{t} \rightarrow l \nu j j b \bar{b}$ & 0.98 & 0.88 & 0.82 \\
\hline$t \bar{t} \rightarrow l \nu l \nu b \bar{b}$ & 1.3 & 1.1 & 0.98 \\
\hline$q t b \rightarrow q \mu \nu b \bar{b}$ & 0.56 & 0.48 & 0.40 \\
\hline$W^{*} \rightarrow t b \rightarrow \mu \nu b \bar{b}$ & 0.45 & 0.38 & 0.33 \\
\hline$W b \bar{b} \rightarrow \mu \nu b \bar{b}$ & 1.3 & 1.1 & 1.0 \\
\hline$Z b \bar{b} \rightarrow \mu \mu b \bar{b}$ & 0.21 & 0.17 & 0.15 \\
\hline non- $W / Z$ & 1.7 & 0.96 & 0.54 \\
\hline Total Background & 14 & 12 & 10.0 \\
\hline Data & 12 & 12 & 11 \\
\hline 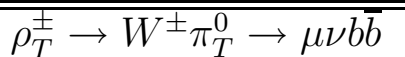 & 0.98 & 0.89 & 0.83 \\
\hline$\rho_{T}^{0} \rightarrow W^{ \pm} \pi_{T}^{\mp} \rightarrow \mu \nu b \bar{c}$ & 1.4 & 1.3 & 1.2 \\
\hline Total Signal & 2.4 & 2.2 & 2.1 \\
\hline
\end{tabular}

Table B.17: Evolution of event yields with additional selection requirements at $M_{\rho}=255 \mathrm{GeV} / c^{2}, M_{\pi}=130 \mathrm{GeV} / c^{2}$, and $M_{V}=100 \mathrm{GeV}$. 


\begin{tabular}{|l|lll|}
\hline sample & $m_{j j}$ & $m_{W j j}$ & $\Delta \phi(j, j)$ \\
\hline \hline$W \rightarrow \mu \nu$ & 0.82 & 0.48 & 0.47 \\
$W \rightarrow \tau \nu$ & & & \\
$Z / \gamma^{*} \rightarrow \mu \mu(60-130)$ & 0.030 & 0.020 & 0.020 \\
$Z / \gamma^{*} \rightarrow \tau \tau(60-130)$ & 0.010 & 0 & 0 \\
$Z / \gamma^{*} \rightarrow \mu \mu(60-130)$ & 0 & 0 & 0 \\
$Z / \gamma^{*} \rightarrow \tau \tau(60-130)$ & & & \\
$W j j \rightarrow \mu \nu j j$ & 4.8 & 3.9 & 3.2 \\
$Z j j \rightarrow \mu \mu j j$ & 0.31 & 0.24 & 0.18 \\
$W W \rightarrow l \nu j j$ & 0.020 & 0.020 & 0.020 \\
$W Z \rightarrow \mu \nu j j$ & 0 & 0 & 0 \\
$W Z \rightarrow j j l l$ & 0 & 0 & 0 \\
$W Z \rightarrow \mu \nu c \bar{c}$ & 0.010 & 0 & 0 \\
$W Z \rightarrow \mu \nu b \bar{b}$ & 0.020 & 0.020 & 0.010 \\
$Z Z \rightarrow \mu \mu j j$ & 0 & 0 & 0 \\
$Z Z \rightarrow \mu \mu c \bar{c}$ & 0 & 0 & 0 \\
$Z Z \rightarrow \mu \mu b \bar{b}$ & 0.010 & 0 & 0 \\
$t \bar{t} \rightarrow l \nu j j b \bar{b}$ & 0.97 & 0.80 & 0.63 \\
$t \bar{t} \rightarrow l \nu l \nu b \bar{b}$ & 1.3 & 0.93 & 0.67 \\
$q t b \rightarrow q \mu \nu b \bar{b}$ & 0.53 & 0.44 & 0.30 \\
$W * \rightarrow t b \rightarrow \mu \nu b \bar{b}$ & 0.41 & 0.35 & 0.27 \\
$W b \bar{b} \rightarrow \mu \nu b \bar{b}$ & 0.91 & 0.70 & 0.59 \\
$Z b \bar{b} \rightarrow \mu \mu b \bar{b}$ & 0.15 & 0.12 & 0.100 \\
non-W/Z & 1.1 & 0.91 & 0.26 \\
\hline Total Background & 11 & 8.9 & 6.7 \\
\hline Data & 12 & 11 & 7 \\
\hline \hline$\rho_{T}^{ \pm} \rightarrow W \pi_{T}^{0} \rightarrow \mu \nu b \bar{b}$ & 1.00 & 0.88 & 0.79 \\
$\rho_{T}^{0} \rightarrow W \pi_{T}^{\mp} \rightarrow \mu \nu b \bar{c}$ & 1.2 & 1.1 & 1.00 \\
\hline Total Signal & 2.2 & 2.0 & 1.8 \\
\hline
\end{tabular}

Table B.18: Evolution of event yields with additional selection requirements at $M_{\rho}=255 \mathrm{GeV} / c^{2}, M_{\pi}=150 \mathrm{GeV} / c^{2}$, and $M_{V}=100 \mathrm{GeV}$. 


\begin{tabular}{|l|lll|}
\hline sample & $m_{j j}$ & $m_{W j j}$ & $\Delta \phi(j, j)$ \\
\hline \hline$W \rightarrow \mu \nu$ & 0.88 & 0.75 & 0.74 \\
$W \rightarrow \tau \nu$ & & & \\
$Z / \gamma^{*} \rightarrow \mu \mu(60-130)$ & 0.030 & 0.030 & 0.020 \\
$Z / \gamma^{*} \rightarrow \tau \tau(60-130)$ & 0 & 0 & 0 \\
$Z / \gamma^{*} \rightarrow \mu \mu(60-130)$ & 0 & 0 & 0 \\
$Z / \gamma^{*} \rightarrow \tau \tau(60-130)$ & & & \\
$W j j \rightarrow \mu \nu j j$ & 4.6 & 3.9 & 3.4 \\
$Z j j \rightarrow \mu \mu j j$ & 0.29 & 0.25 & 0.22 \\
$W W \rightarrow l \nu j j$ & 0.020 & 0.020 & 0.020 \\
$W Z \rightarrow \mu \nu j j$ & 0 & 0 & 0 \\
$W Z \rightarrow j j l l$ & 0 & 0 & 0 \\
$W Z \rightarrow \mu \nu c \bar{c}$ & 0.010 & 0 & 0 \\
$W Z \rightarrow \mu \nu b \bar{b}$ & 0.020 & 0.020 & 0.020 \\
$Z Z \rightarrow \mu \mu j j$ & 0 & 0 & 0 \\
$Z Z \rightarrow \mu \mu c \bar{c}$ & 0 & 0 & 0 \\
$Z Z \rightarrow \mu \mu b \bar{b}$ & 0.010 & 0.010 & 0 \\
$t \bar{t} \rightarrow l \nu j j \bar{b} \bar{t}$ & 0.86 & 0.80 & 0.76 \\
$t \bar{t} \rightarrow l \nu l \nu b \bar{b}$ & 1.2 & 1.0 & 0.91 \\
$q t b \rightarrow q \mu \nu b \bar{b}$ & 0.47 & 0.43 & 0.35 \\
$W * \rightarrow t b \rightarrow \mu \nu b \bar{b}$ & 0.36 & 0.32 & 0.29 \\
$W b \bar{b} \rightarrow \mu \nu b \bar{b}$ & 0.87 & 0.75 & 0.70 \\
$Z b \bar{b} \rightarrow \mu \mu b \bar{b}$ & 0.14 & 0.12 & 0.11 \\
non-W/Z & 0.91 & 0.79 & 0.36 \\
\hline Total Background & 11 & 9.2 & 7.9 \\
\hline Data & 11 & 11 & 10 \\
\hline \hline$\rho_{T}^{ \pm} \rightarrow W \pi_{T}^{0} \rightarrow \mu \nu b \bar{b}$ & 0.76 & 0.71 & 0.66 \\
$\rho_{T}^{0} \rightarrow W \pi_{T}^{\mp} \rightarrow \mu \nu b \bar{c}$ & 1.2 & 1.1 & 1.0 \\
\hline Total Signal & 1.9 & 1.8 & 1.7 \\
\hline
\end{tabular}

Table B.19: Evolution of event yields with additional selection requirements at $M_{\rho}=260 \mathrm{GeV} / c^{2}, M_{\pi}=140 \mathrm{GeV} / c^{2}$, and $M_{V}=100 \mathrm{GeV}$. 


\begin{tabular}{|l|lll|}
\hline sample & $m_{j j}$ & $m_{W j j}$ & $\Delta \phi(j, j)$ \\
\hline \hline$W \rightarrow \mu \nu$ & 0.88 & 0.75 & 0.74 \\
$W \rightarrow \tau \nu$ & & & \\
$Z / \gamma^{*} \rightarrow \mu \mu(60-130)$ & 0.030 & 0.010 & 0.010 \\
$Z / \gamma^{*} \rightarrow \tau \tau(60-130)$ & 0.010 & 0 & 0 \\
$Z / \gamma^{*} \rightarrow \mu \mu(60-130)$ & 0 & 0 & 0 \\
$Z / \gamma^{*} \rightarrow \tau \tau(60-130)$ & & & \\
$W j j \rightarrow \mu \nu j j$ & 5.0 & 4.2 & 3.7 \\
$Z j j \rightarrow \mu \mu j j$ & 0.31 & 0.27 & 0.25 \\
$W W \rightarrow l \nu j j$ & 0.020 & 0.020 & 0.010 \\
$W Z \rightarrow \mu \nu j j$ & 0 & 0 & 0 \\
$W Z \rightarrow j j l l$ & 0 & 0 & 0 \\
$W Z \rightarrow \mu \nu c \bar{c}$ & 0.010 & 0 & 0 \\
$W Z \rightarrow \mu \nu b \bar{b}$ & 0.020 & 0.020 & 0.020 \\
$Z Z \rightarrow \mu \mu j j$ & 0 & 0 & 0 \\
$Z Z \rightarrow \mu \mu c \bar{c}$ & 0 & 0 & 0 \\
$Z Z \rightarrow \mu \mu b \bar{b}$ & 0.010 & 0.010 & 0.010 \\
$t \bar{t} \rightarrow l \nu j j b \bar{b}$ & 1.00 & 0.90 & 0.87 \\
$t \bar{t} \rightarrow l \nu l \nu b \bar{b}$ & 1.4 & 1.2 & 1.1 \\
$q t b \rightarrow q \mu \nu b \bar{b}$ & 0.55 & 0.49 & 0.43 \\
$W^{*} \rightarrow t b \rightarrow \mu \nu b \bar{b}$ & 0.42 & 0.36 & 0.34 \\
$W b \bar{b} \rightarrow \mu \nu b \bar{b}$ & 0.94 & 0.76 & 0.72 \\
$Z b \bar{b} \rightarrow \mu \mu b \bar{b}$ & 0.16 & 0.13 & 0.12 \\
non-W/Z & 1.1 & 0.91 & 0.60 \\
\hline Total Background & 12 & 10 & 8.9 \\
\hline Data & 12 & 11 & 11 \\
\hline \hline$\rho_{T}^{ \pm} \rightarrow W \pi_{T}^{0} \rightarrow \mu \nu b \bar{b}$ & 0.70 & 0.65 & 0.61 \\
$\rho_{T}^{0} \rightarrow W \pi_{T}^{\mp} \rightarrow \mu \nu b \bar{c}$ & 1.2 & 1.1 & 1.0 \\
\hline Total Signal & 1.9 & 1.7 & 1.6 \\
\hline
\end{tabular}

Table B.20: Evolution of event yields with additional selection requirements at $M_{\rho}=275 \mathrm{GeV} / c^{2}, M_{\pi}=150 \mathrm{GeV} / c^{2}$, and $M_{V}=100 \mathrm{GeV}$. 


\section{Appendix C}

\section{Cut Variable Distributions}

Distributions of $M_{j j}, M_{W j j}$ and $\Delta \phi(j, j)$ for each mass point are shown. The distributions are after all selection criteria applied except for the requirement on the variable plotted. The mass-dependent criteria for each variable are detailed in table 6.7. In all cases the parameter $M_{V}$ is set to $100 \mathrm{GeV}$. 

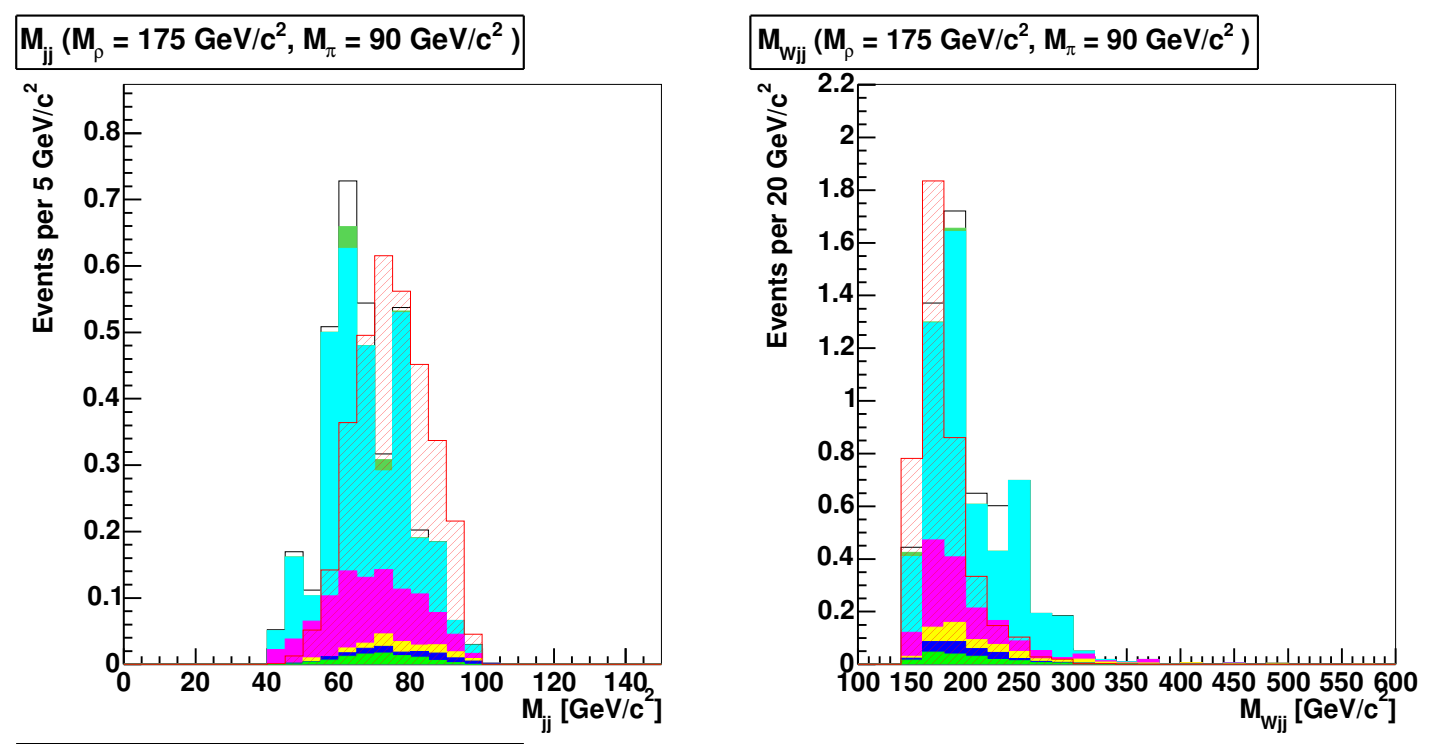

$\Delta \Phi(\mathrm{j}, \mathrm{j})\left(\mathrm{M}_{\rho}=175 \mathrm{GeV} / \mathrm{c}^{2}, \mathrm{M}_{\mathrm{r}}=90 \mathrm{GeV} / \mathrm{c}^{2}\right)$

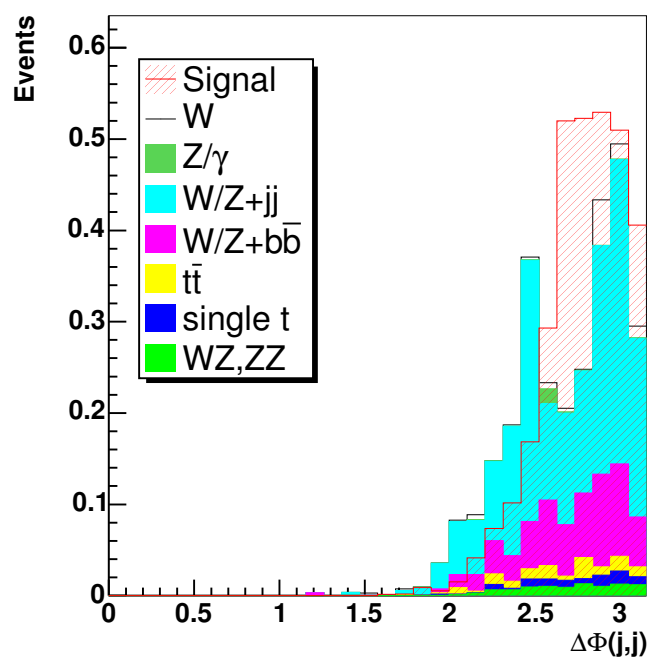

Figure C.1: Signal and background distributions of selection variables at $M_{\rho}=$ $175 \mathrm{GeV} / c^{2}, M_{\pi}=90 \mathrm{GeV} / c^{2}$, and $M_{V}=100 \mathrm{GeV}$. 

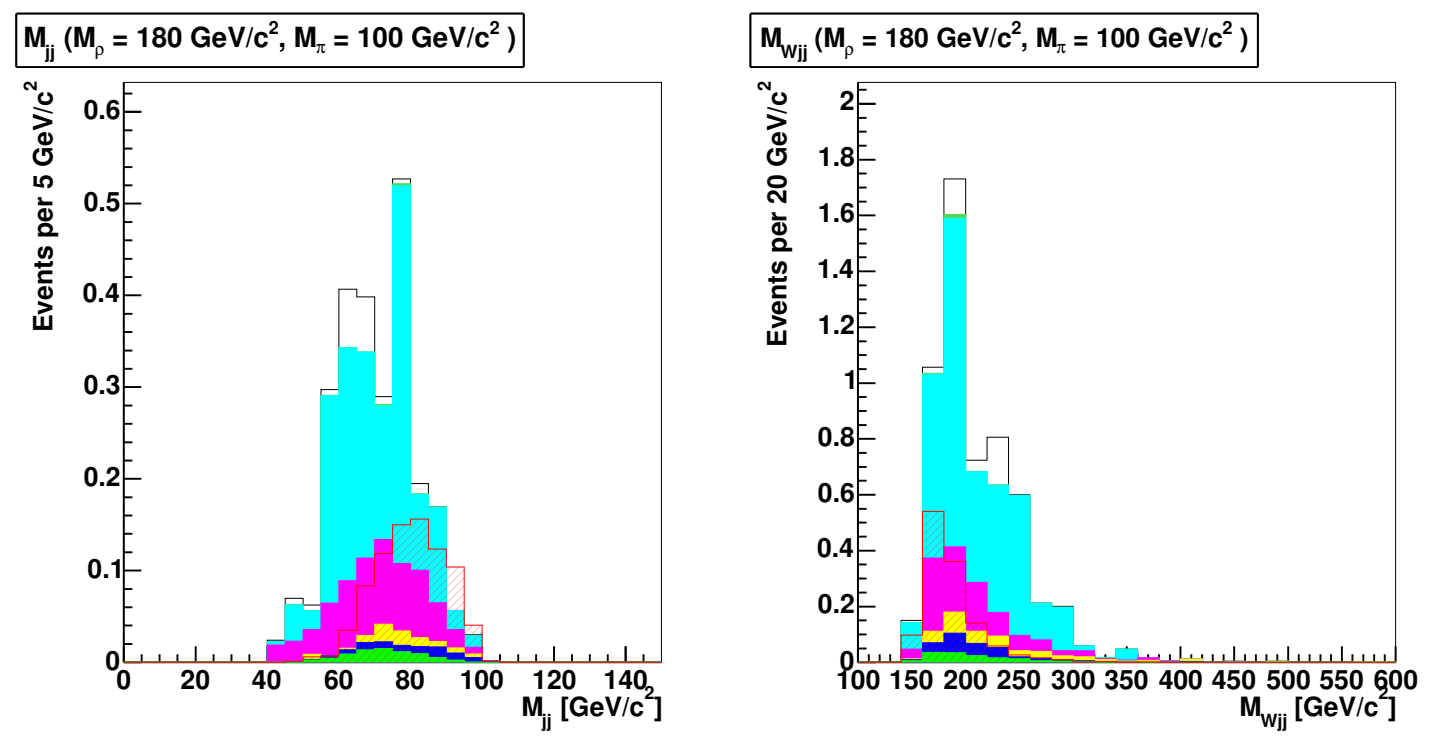

$\Delta \Phi(\mathrm{j}, \mathrm{j})\left(\mathrm{M}_{\rho}=180 \mathrm{GeV} / \mathrm{c}^{2}, \mathrm{M}_{\pi}=100 \mathrm{GeV} / \mathrm{c}^{2}\right)$

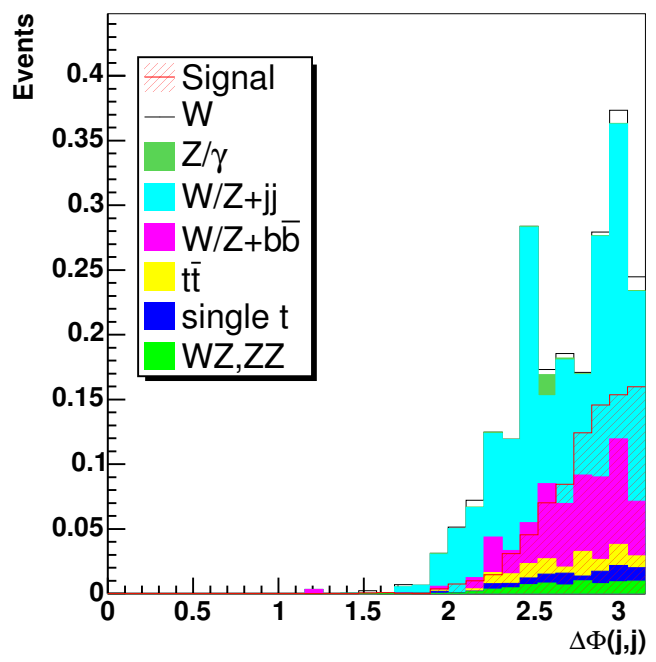

Figure C.2: Signal and background distributions of selection variables at $M_{\rho}=$ $180 \mathrm{GeV} / c^{2}, M_{\pi}=100 \mathrm{GeV} / c^{2}$, and $M_{V}=100 \mathrm{GeV}$. 

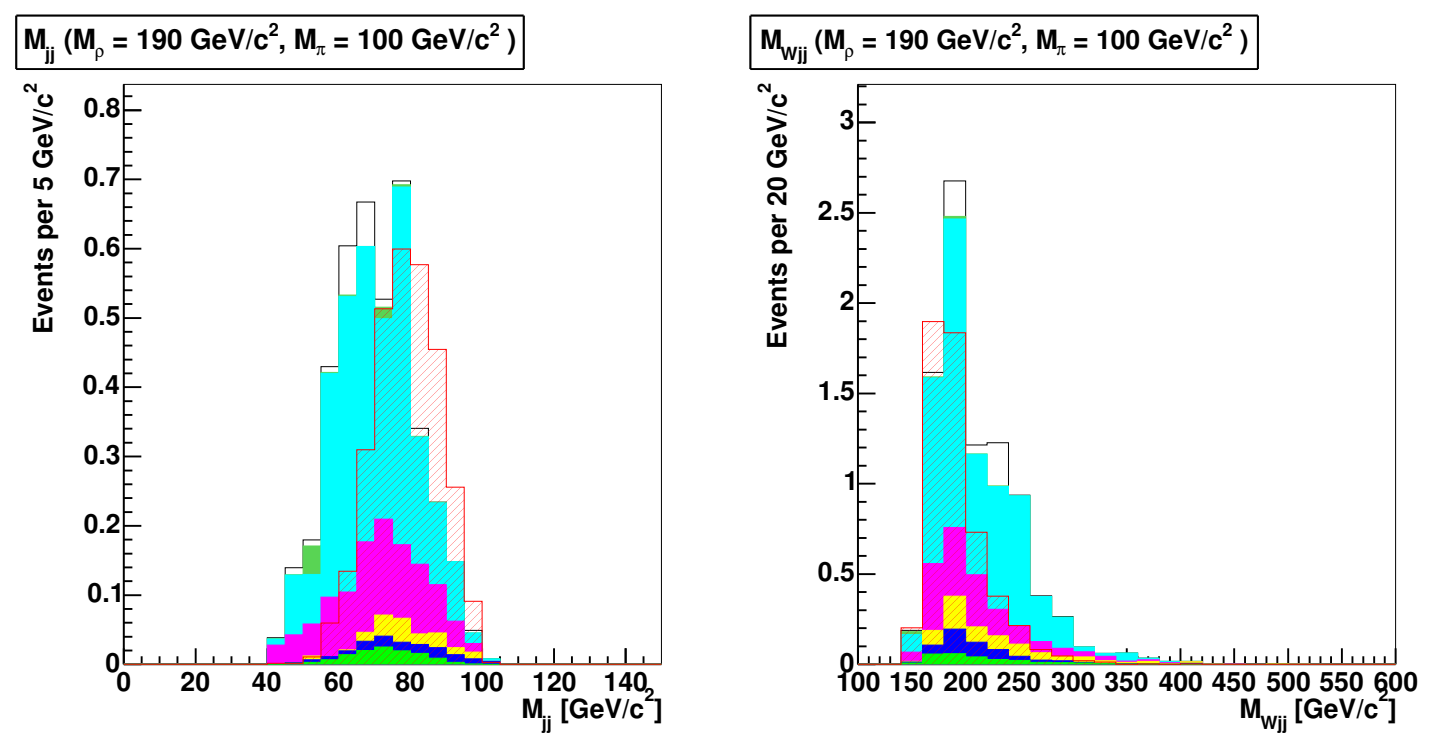

$\Delta \Phi(\mathrm{j}, \mathrm{j})\left(\mathrm{M}_{\rho}=190 \mathrm{GeV} / \mathrm{c}^{2}, \mathrm{M}_{\pi}=100 \mathrm{GeV} / \mathrm{c}^{2}\right)$

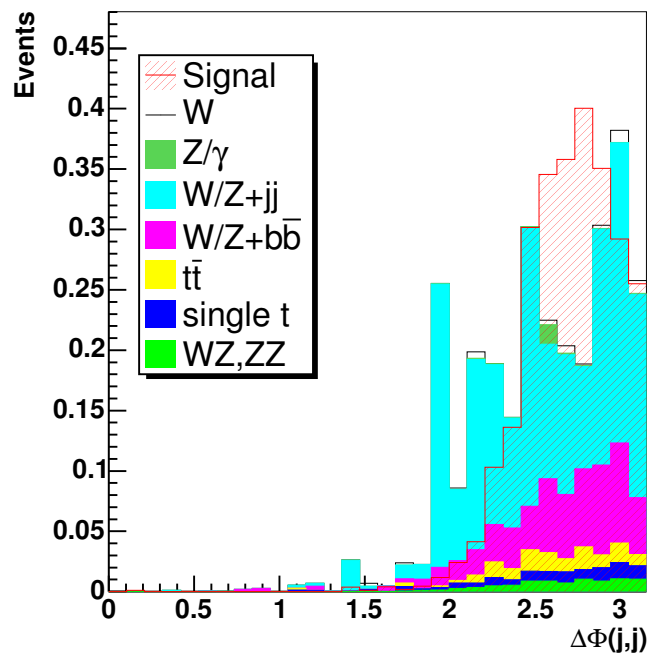

Figure C.3: Signal and background distributions of selection variables at $M_{\rho}=$ $190 \mathrm{GeV} / c^{2}, M_{\pi}=100 \mathrm{GeV} / c^{2}$, and $M_{V}=100 \mathrm{GeV}$. 

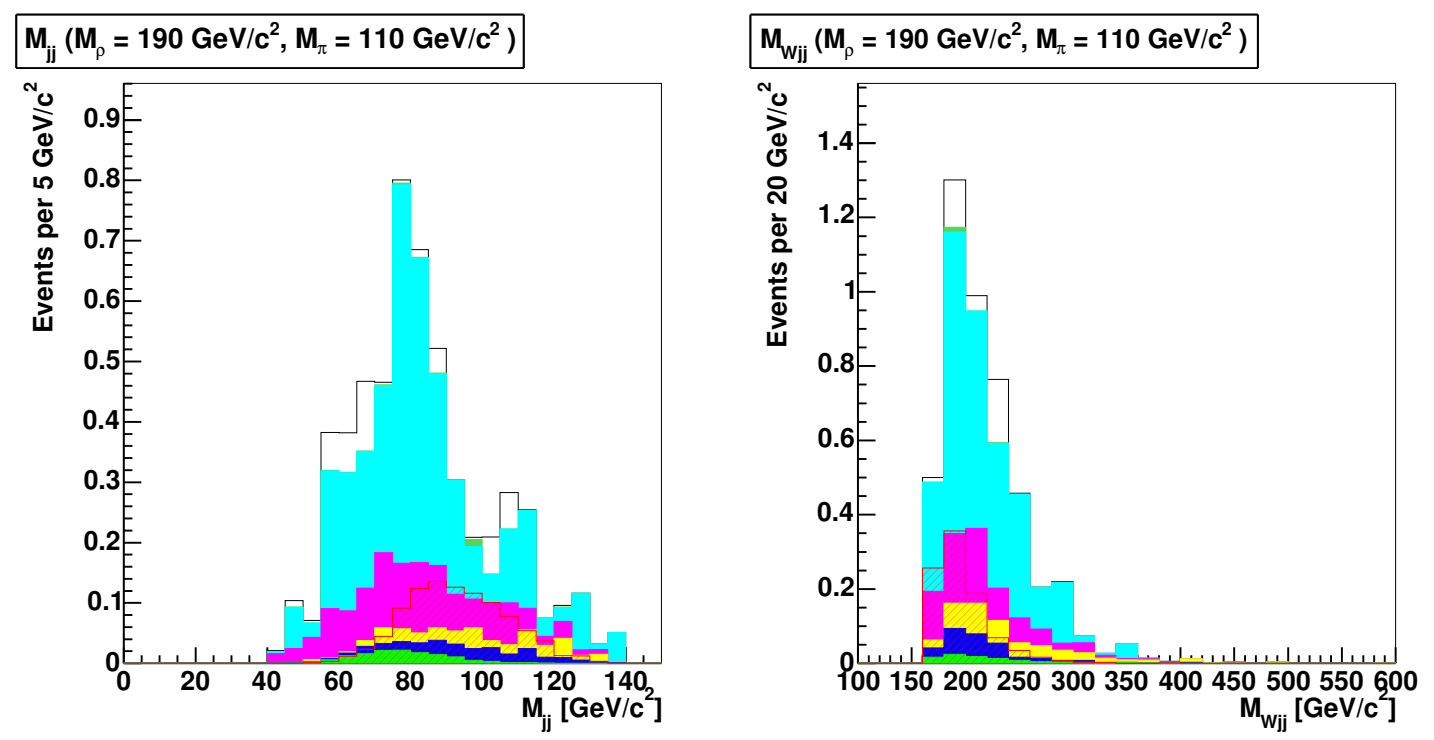

$\Delta \Phi(\mathrm{j}, \mathrm{j})\left(\mathrm{M}_{\mathrm{\rho}}=190 \mathrm{GeV} / \mathrm{c}^{2}, \mathrm{M}_{\pi}=110 \mathrm{GeV} / \mathrm{c}^{2}\right)$

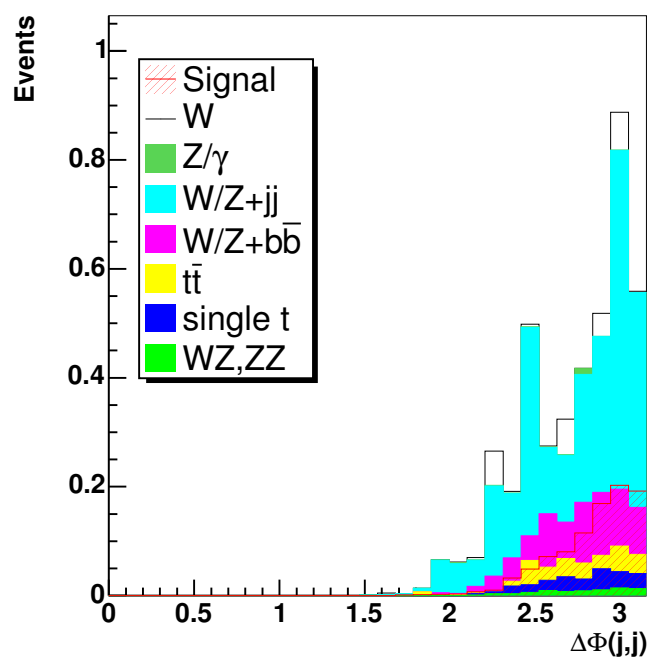

Figure C.4: Signal and background distributions of selection variables at $M_{\rho}=$ $190 \mathrm{GeV} / c^{2}, M_{\pi}=110 \mathrm{GeV} / c^{2}$, and $M_{V}=100 \mathrm{GeV}$. 

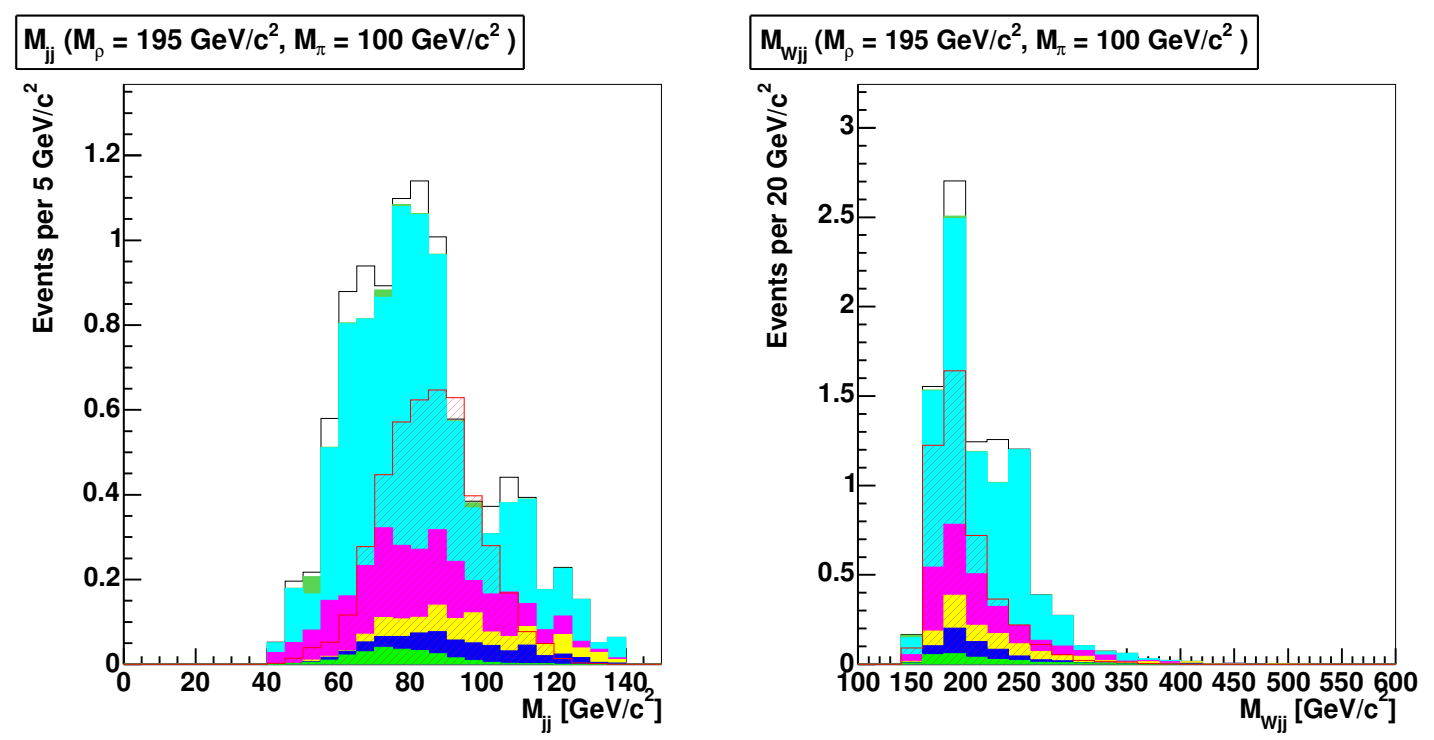

$\Delta \Phi(\mathrm{j}, \mathrm{j})\left(\mathrm{M}_{\rho}=195 \mathrm{GeV} / \mathrm{c}^{2}, \mathrm{M}_{\pi}=100 \mathrm{GeV} / \mathrm{c}^{2}\right)$

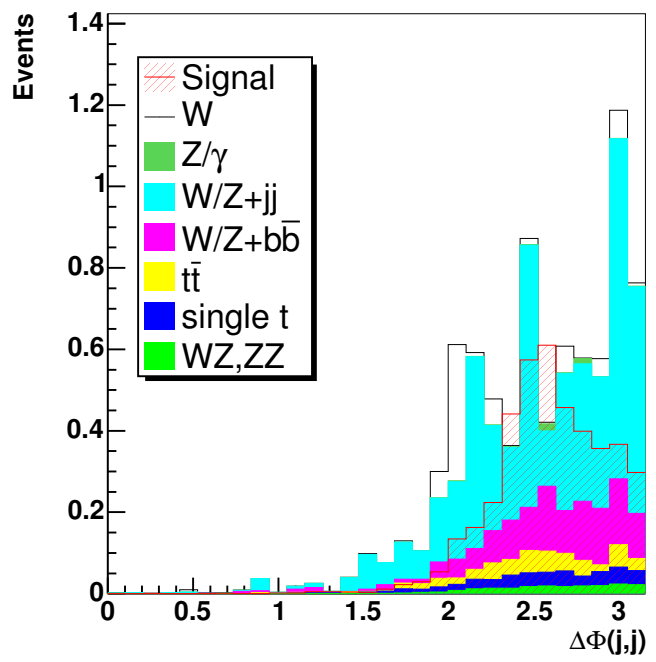

Figure C.5: Signal and background distributions of selection variables at $M_{\rho}=$ $195 \mathrm{GeV} / c^{2}, M_{\pi}=100 \mathrm{GeV} / c^{2}$, and $M_{V}=100 \mathrm{GeV}$. 

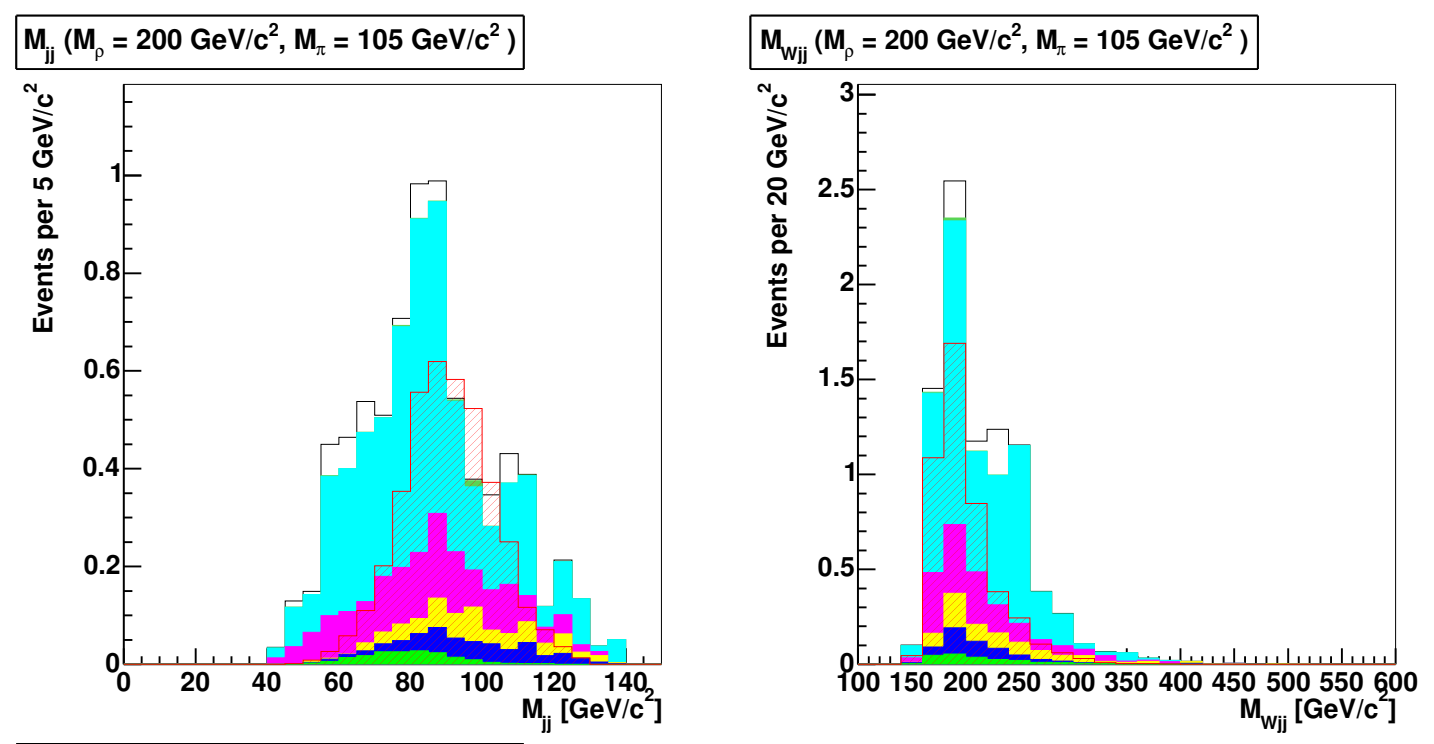

$\Delta \Phi(\mathrm{j}, \mathrm{j})\left(\mathrm{M}_{\rho}=200 \mathrm{GeV} / \mathrm{c}^{2}, \mathrm{M}_{\pi}=105 \mathrm{GeV} / \mathrm{c}^{2}\right)$

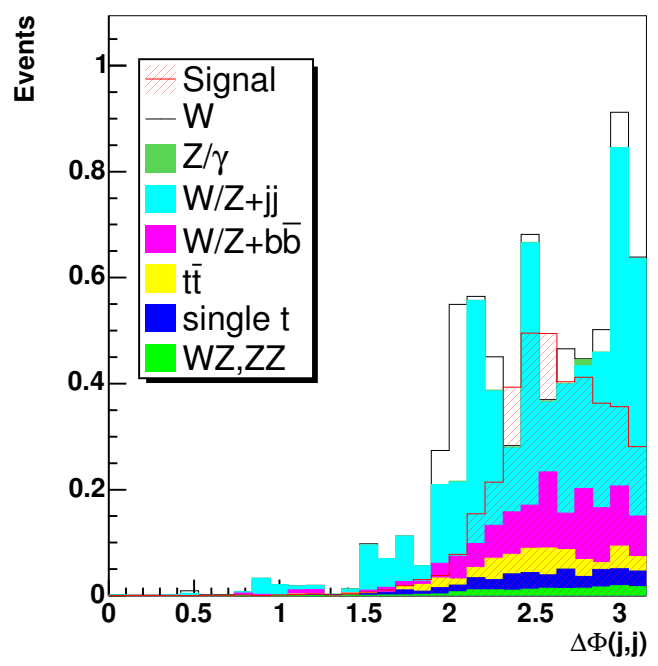

Figure C.6: Signal and background distributions of selection variables at $M_{\rho}=$ $200 \mathrm{GeV} / c^{2}, M_{\pi}=105 \mathrm{GeV} / c^{2}$, and $M_{V}=100 \mathrm{GeV}$. 

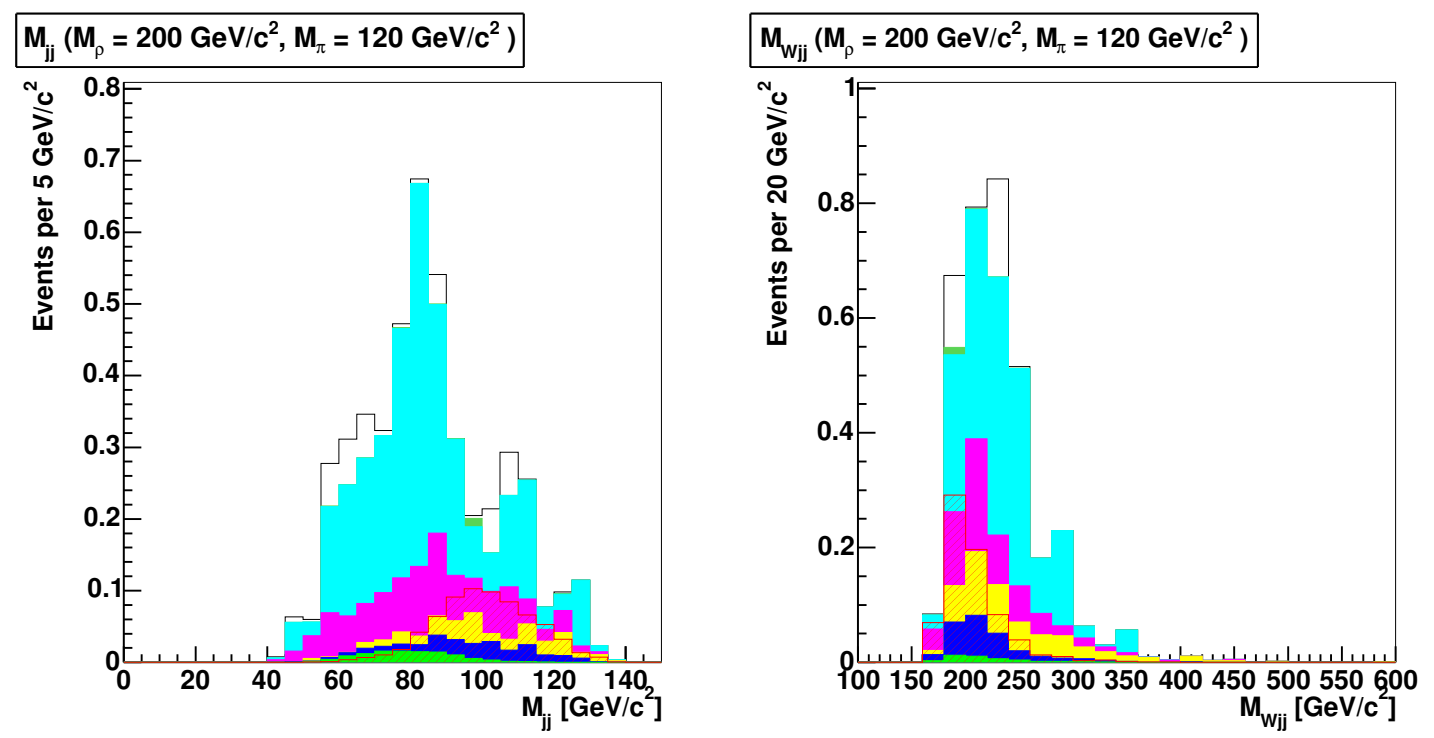

$\Delta \Phi(j, j)\left(M_{p}=200 \mathrm{GeV} / \mathrm{c}^{2}, M_{\pi}=120 \mathrm{GeV} / \mathrm{c}^{2}\right)$

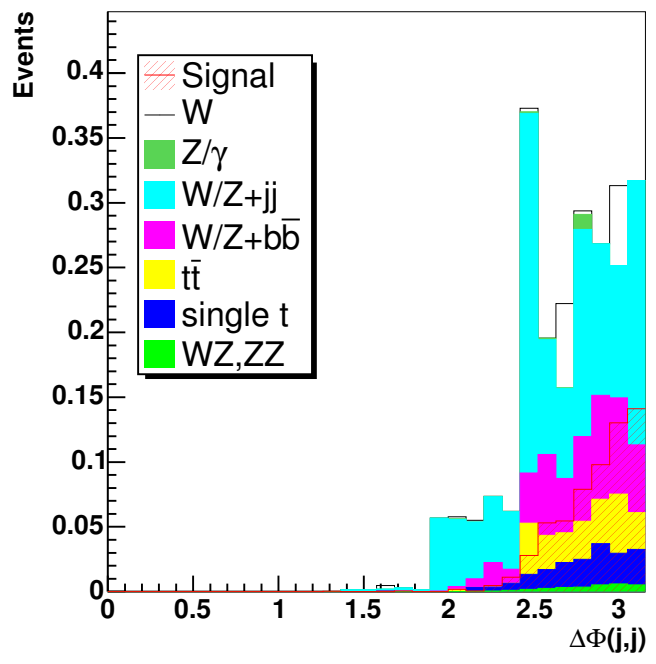

Figure C.7: Signal and background distributions of selection variables at $M_{\rho}=$ $200 \mathrm{GeV} / c^{2}, M_{\pi}=120 \mathrm{GeV} / c^{2}$, and $M_{V}=100 \mathrm{GeV}$. 

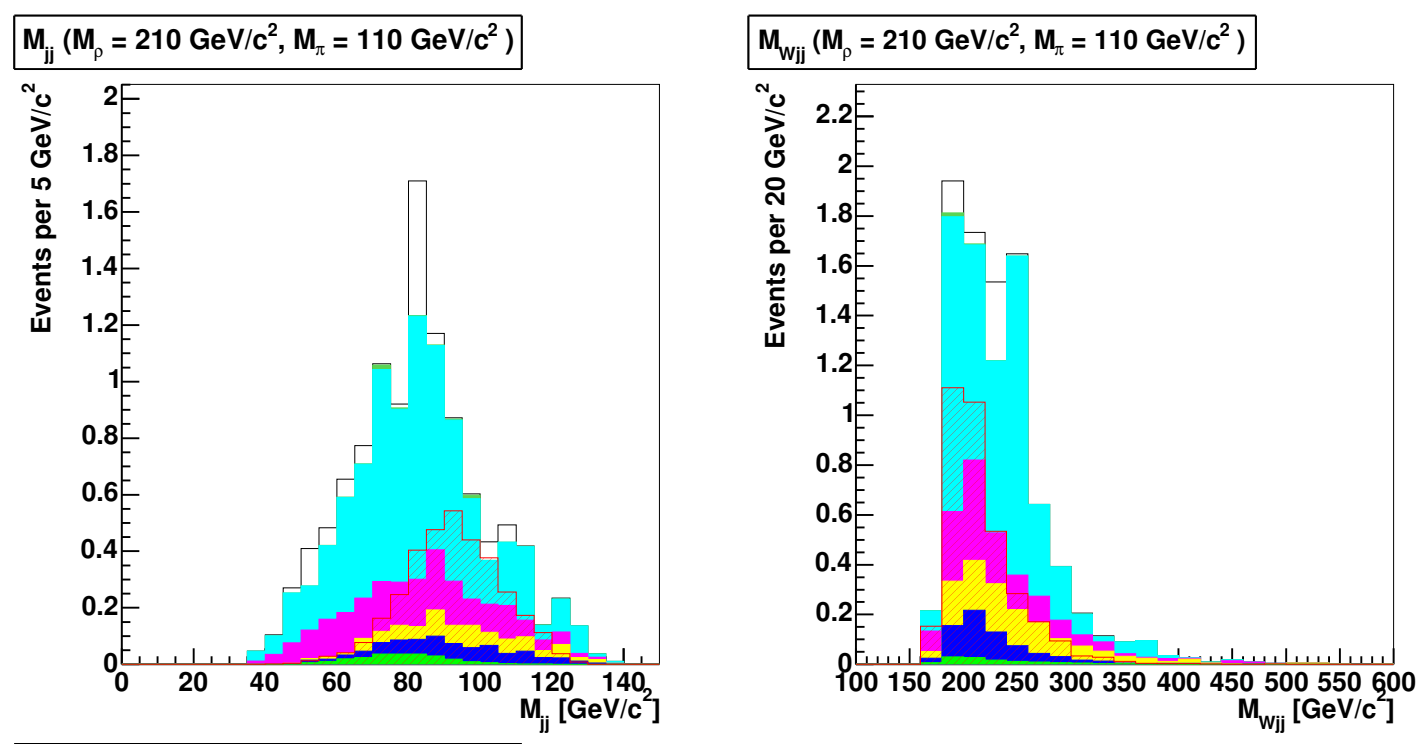

$\Delta \Phi(\mathrm{j}, \mathrm{j})\left(\mathrm{M}_{\rho}=210 \mathrm{GeV} / \mathrm{c}^{2}, \mathrm{M}_{\pi}=110 \mathrm{GeV} / \mathrm{c}^{2}\right)$

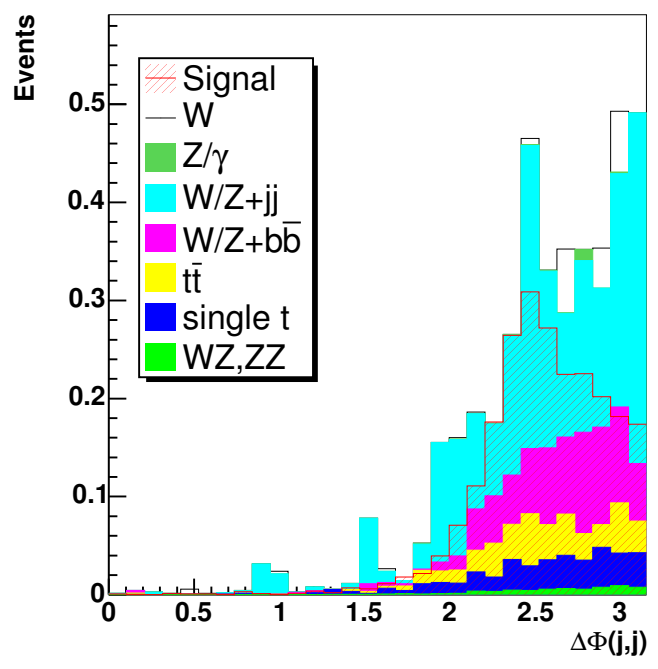

Figure C.8: Signal and background distributions of selection variables at $M_{\rho}=$ $210 \mathrm{GeV} / c^{2}, M_{\pi}=110 \mathrm{GeV} / c^{2}$, and $M_{V}=100 \mathrm{GeV}$. 

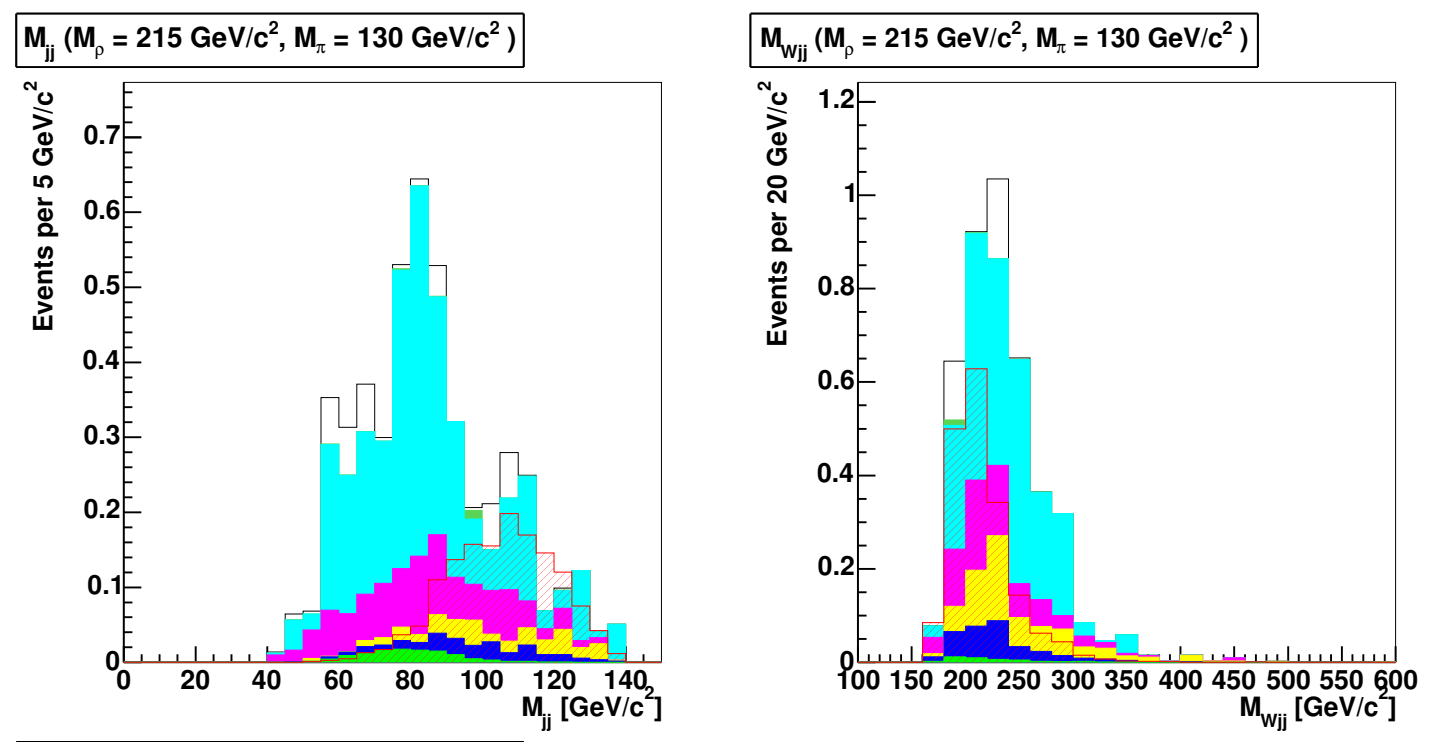

$\Delta \Phi(\mathrm{j}, \mathrm{j})\left(\mathrm{M}_{\rho}=215 \mathrm{GeV} / \mathrm{c}^{2}, \mathrm{M}_{\pi}=130 \mathrm{GeV} / \mathrm{c}^{2}\right)$

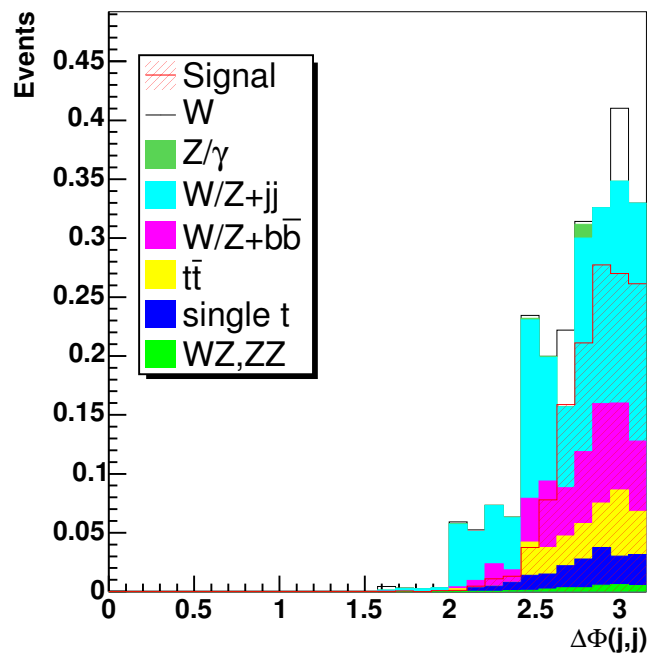

Figure C.9: Signal and background distributions of selection variables at $M_{\rho}=$ $215 \mathrm{GeV} / c^{2}, M_{\pi}=130 \mathrm{GeV} / c^{2}$, and $M_{V}=100 \mathrm{GeV}$. 

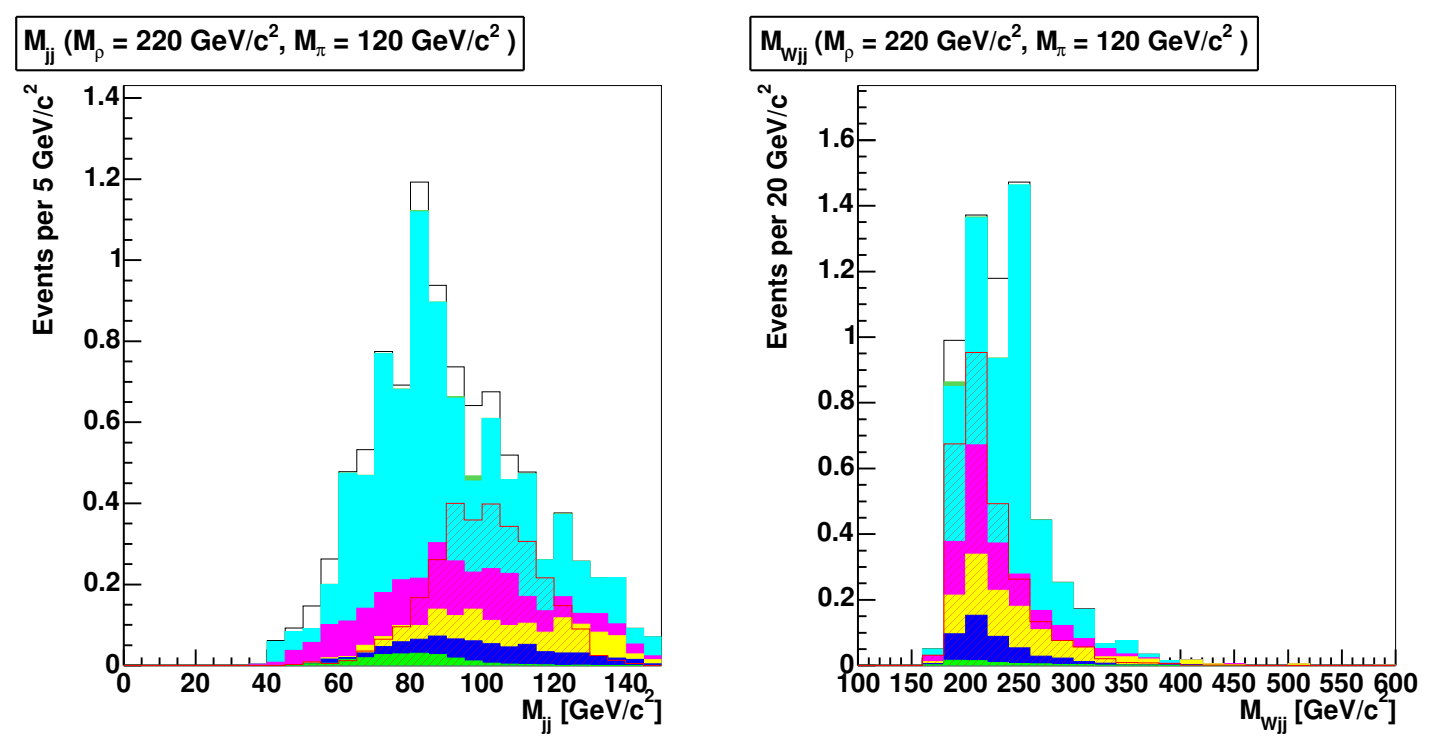

$\Delta \Phi(\mathrm{j}, \mathrm{j})\left(\mathrm{M}_{\mathrm{p}}=220 \mathrm{GeV} / \mathrm{c}^{2}, \mathrm{M}_{\pi}=120 \mathrm{GeV} / \mathrm{c}^{2}\right)$

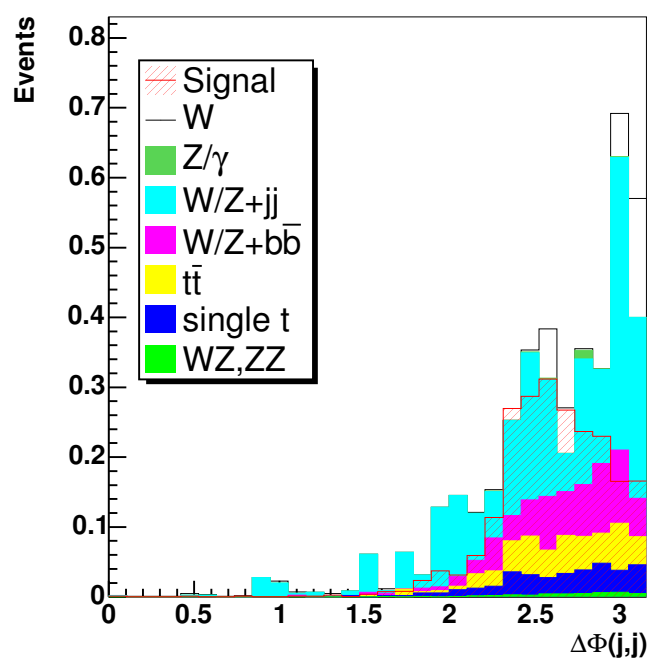

Figure C.10: Signal and background distributions of selection variables at $M_{\rho}=220 \mathrm{GeV} / c^{2}, M_{\pi}=120 \mathrm{GeV} / c^{2}$, and $M_{V}=100 \mathrm{GeV}$. 

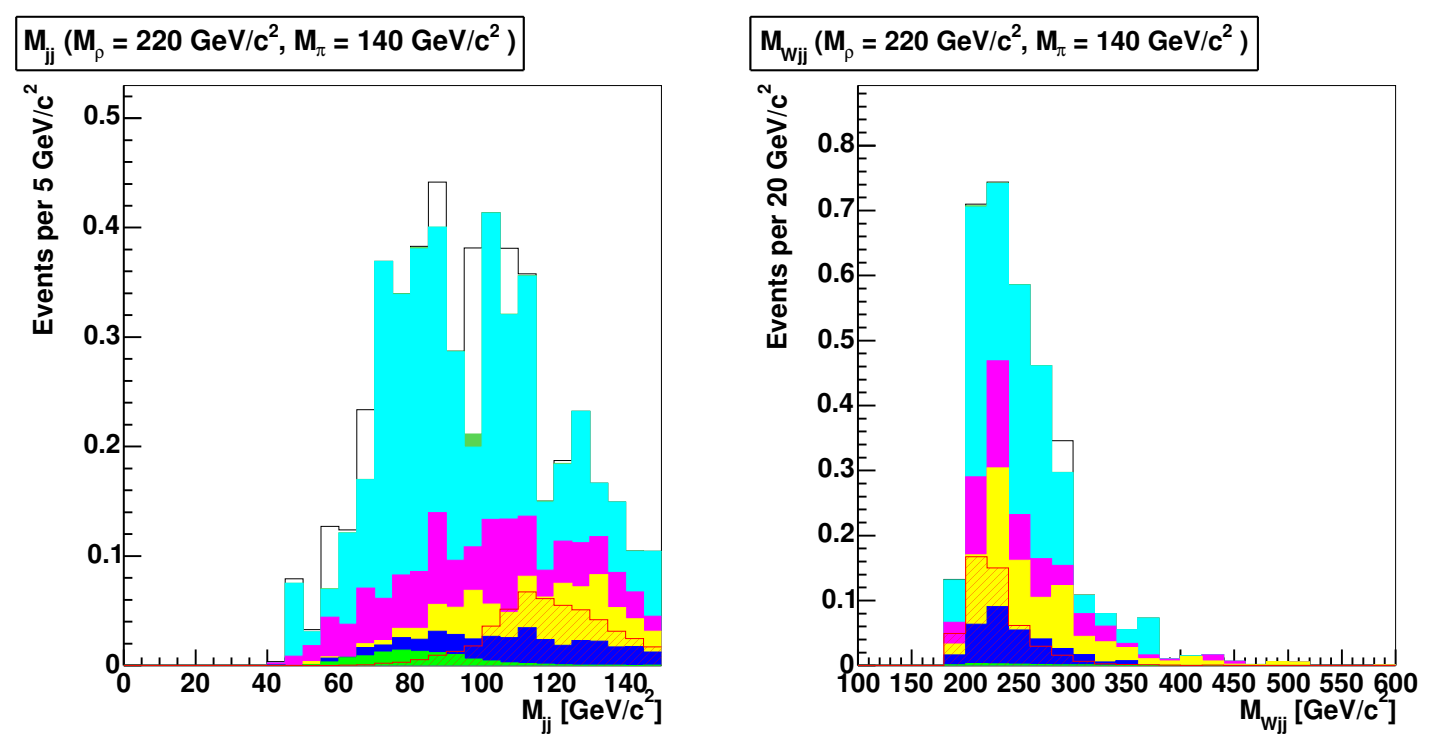

$\Delta \Phi(\mathrm{j}, \mathrm{j})\left(\mathrm{M}_{\mathrm{p}}=220 \mathrm{GeV} / \mathrm{c}^{2}, \mathrm{M}_{\pi}=140 \mathrm{GeV} / \mathrm{c}^{2}\right)$

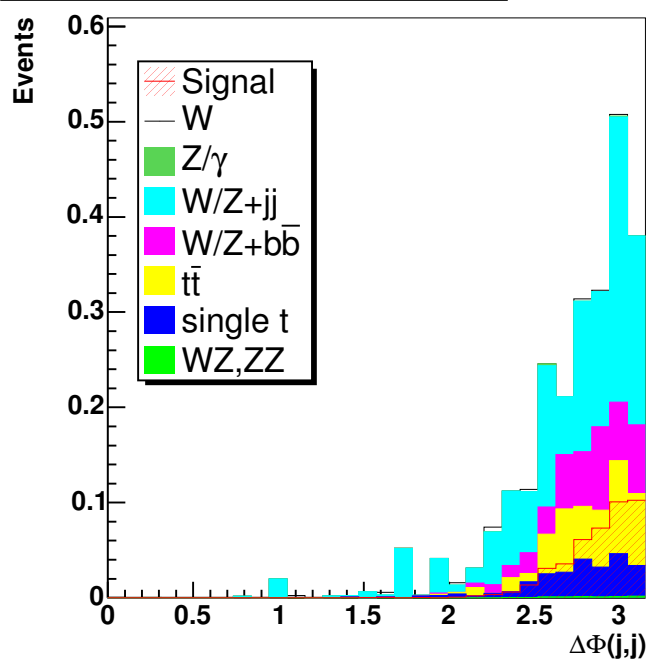

Figure C.11: Signal and background distributions of selection variables at $M_{\rho}=220 \mathrm{GeV} / c^{2}, M_{\pi}=140 \mathrm{GeV} / c^{2}$, and $M_{V}=100 \mathrm{GeV}$. 

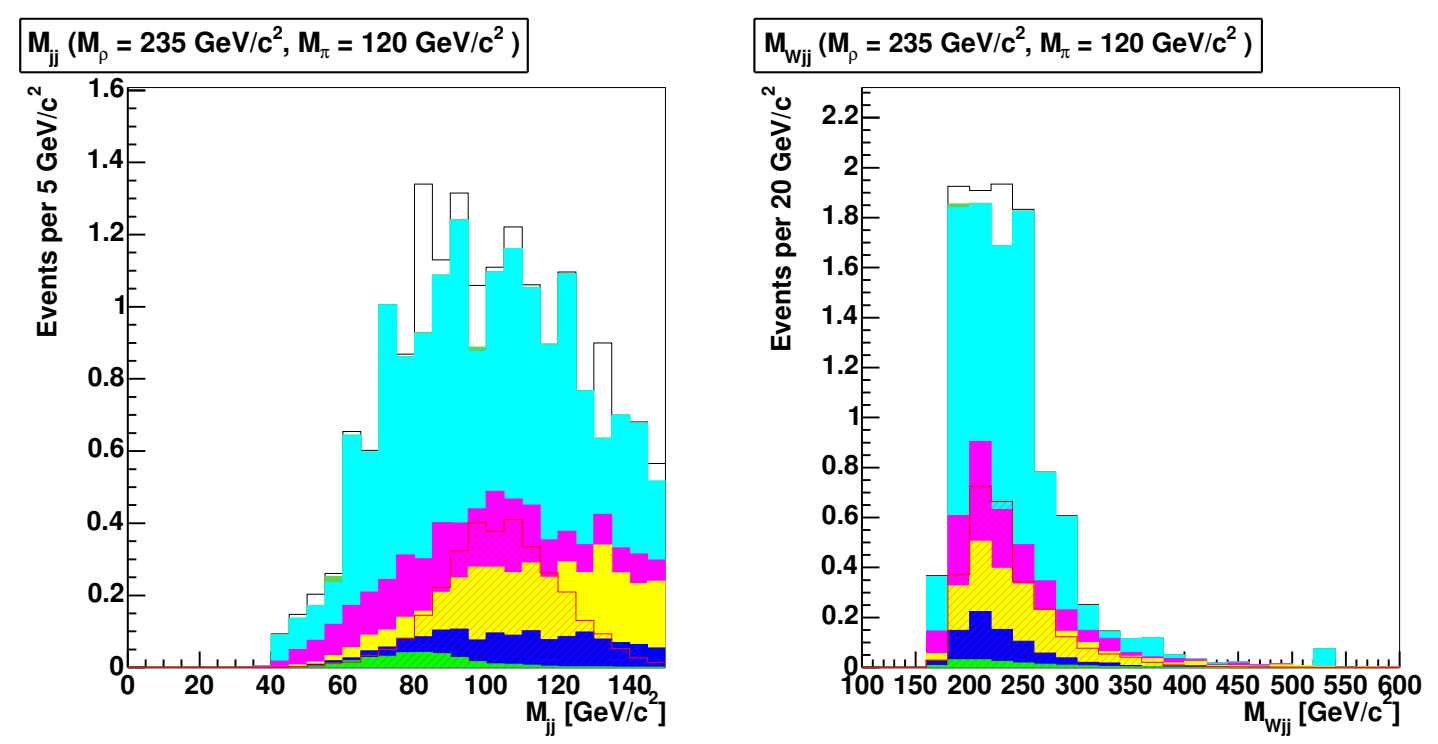

$\Delta \Phi(\mathrm{j}, \mathrm{j})\left(\mathrm{M}_{\mathrm{\rho}}=235 \mathrm{GeV} / \mathrm{c}^{2}, \mathrm{M}_{\pi}=120 \mathrm{GeV} / \mathrm{c}^{2}\right)$

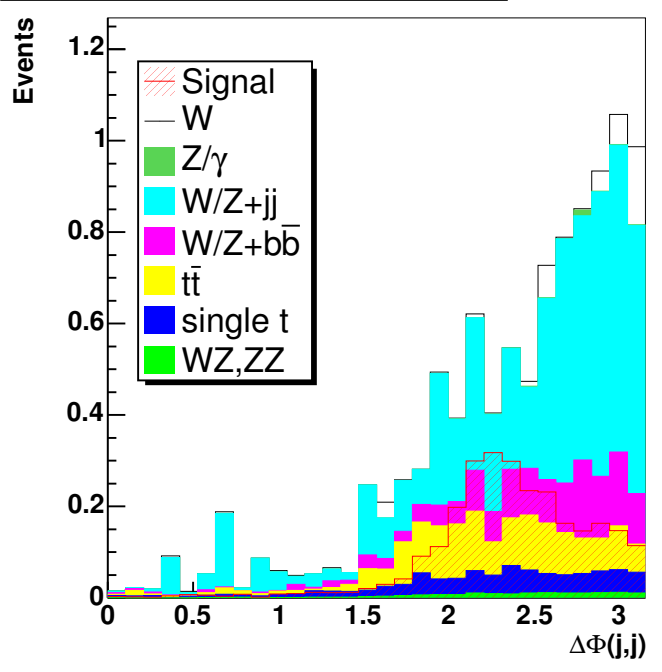

Figure C.12: Signal and background distributions of selection variables at $M_{\rho}=235 \mathrm{GeV} / c^{2}, M_{\pi}=120 \mathrm{GeV} / c^{2}$, and $M_{V}=100 \mathrm{GeV}$. 

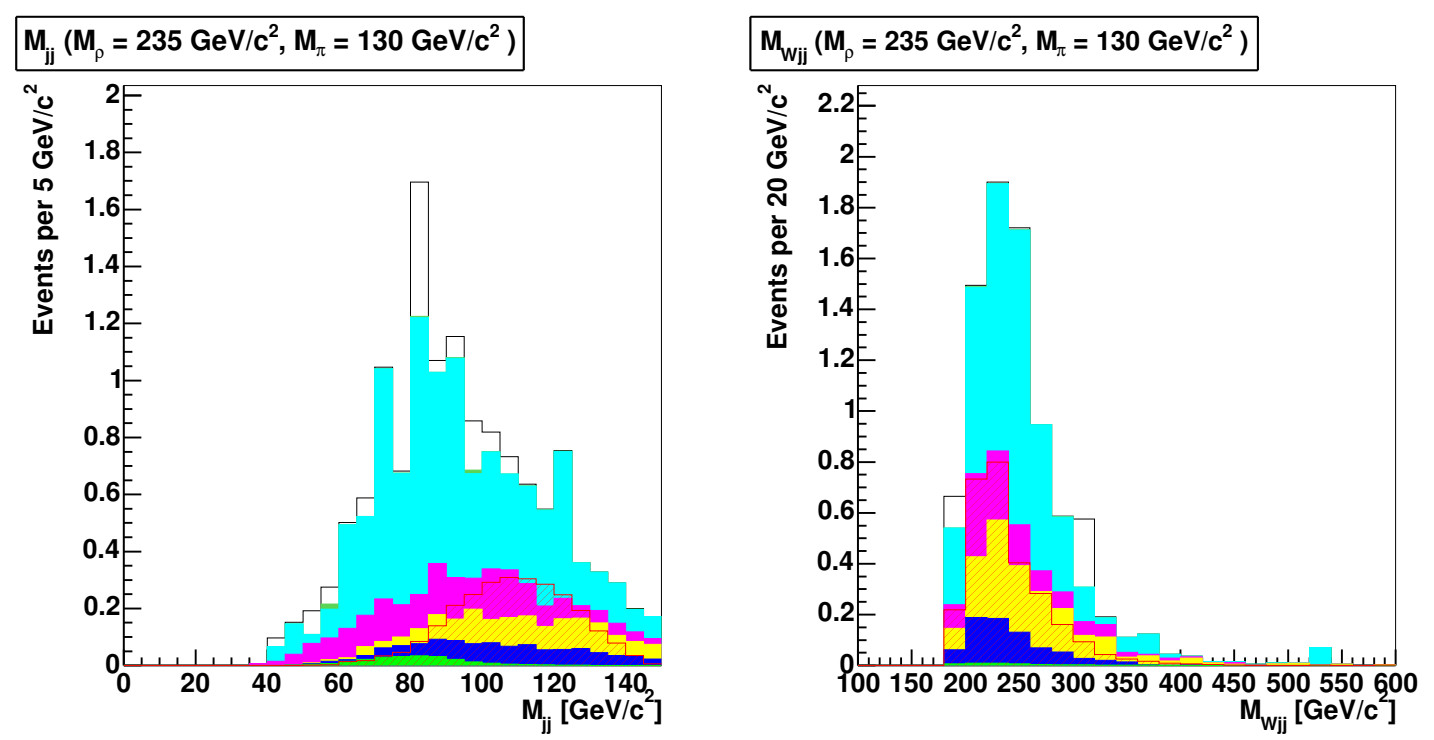

$\Delta \Phi(\mathrm{j}, \mathrm{j})\left(\mathrm{M}_{\mathrm{p}}=235 \mathrm{GeV} / \mathrm{c}^{2}, \mathrm{M}_{\pi}=130 \mathrm{GeV} / \mathrm{c}^{2}\right)$

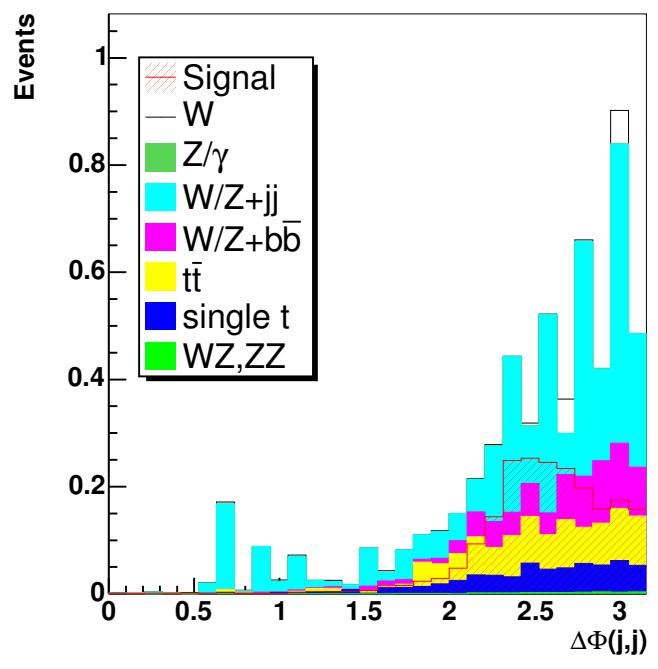

Figure C.13: Signal and background distributions of selection variables at $M_{\rho}=235 \mathrm{GeV} / c^{2}, M_{\pi}=130 \mathrm{GeV} / c^{2}$, and $M_{V}=100 \mathrm{GeV}$. 

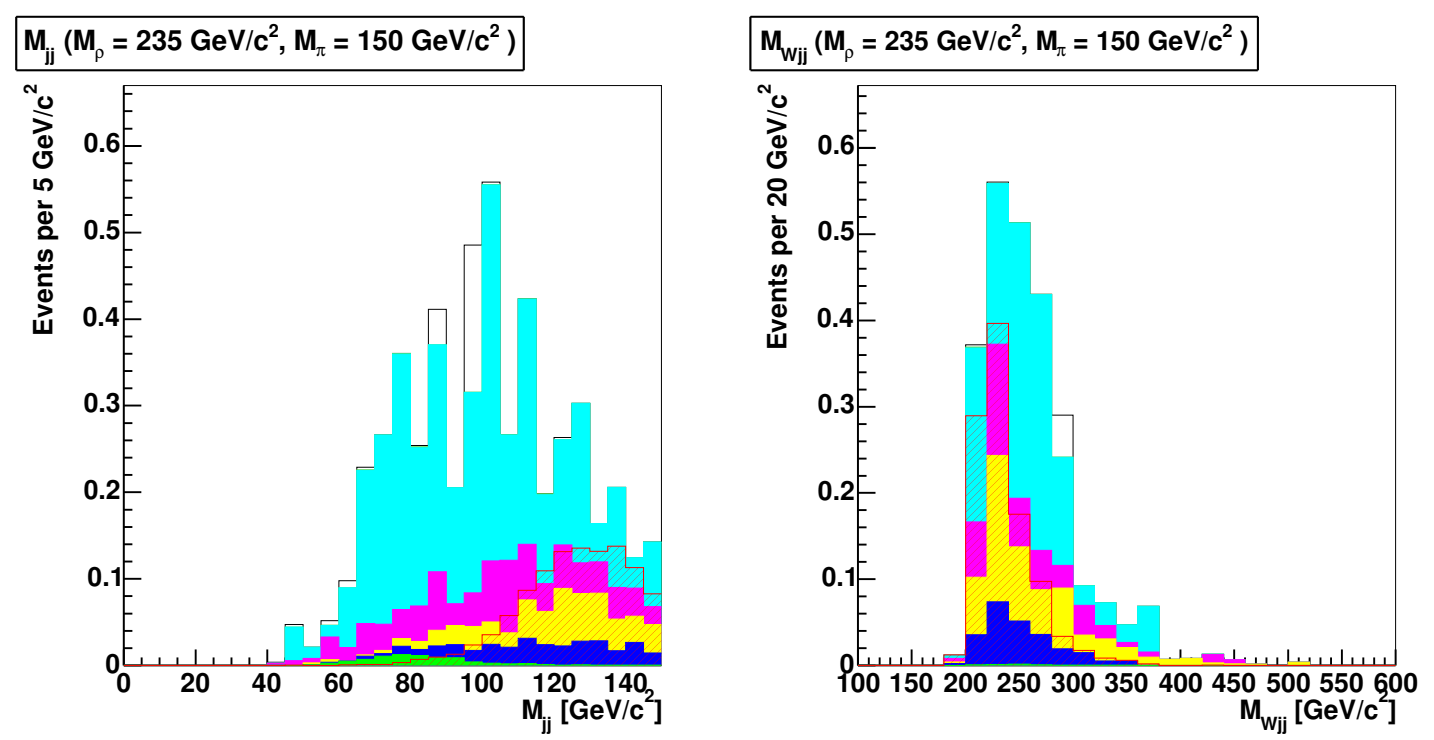

$\Delta \Phi(\mathrm{j}, \mathrm{j})\left(\mathrm{M}_{\mathrm{p}}=235 \mathrm{GeV} / \mathrm{c}^{2}, \mathrm{M}_{\pi}=150 \mathrm{GeV} / \mathrm{c}^{2}\right)$

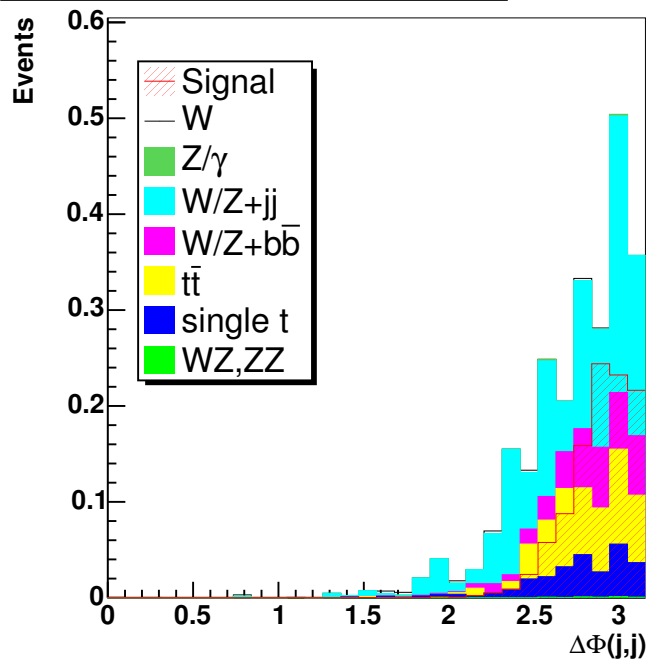

Figure C.14: Signal and background distributions of selection variables at $M_{\rho}=235 \mathrm{GeV} / c^{2}, M_{\pi}=150 \mathrm{GeV} / c^{2}$, and $M_{V}=100 \mathrm{GeV}$. 

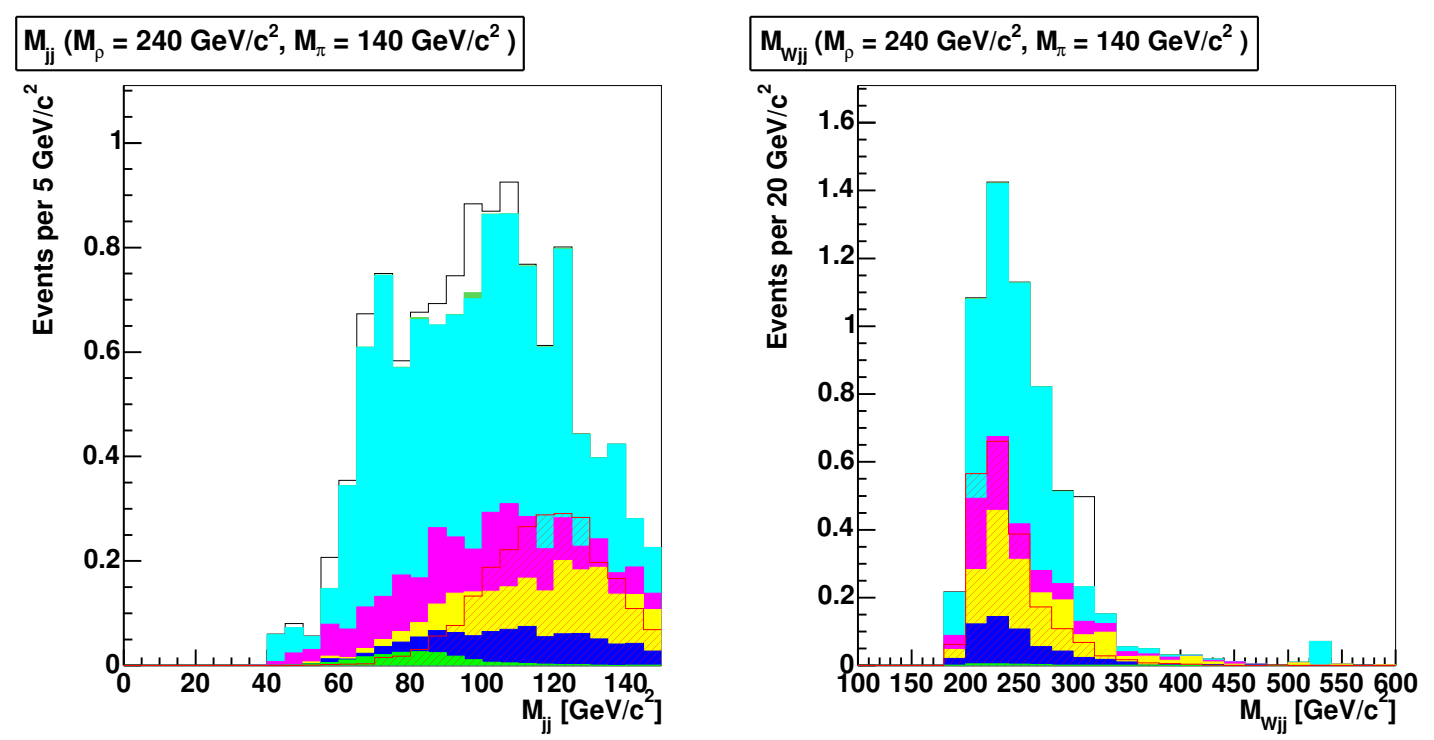

$\Delta \Phi(\mathrm{j}, \mathrm{j})\left(\mathrm{M}_{\mathrm{p}}=240 \mathrm{GeV} / \mathrm{c}^{2}, \mathrm{M}_{\pi}=140 \mathrm{GeV} / \mathrm{c}^{2}\right)$

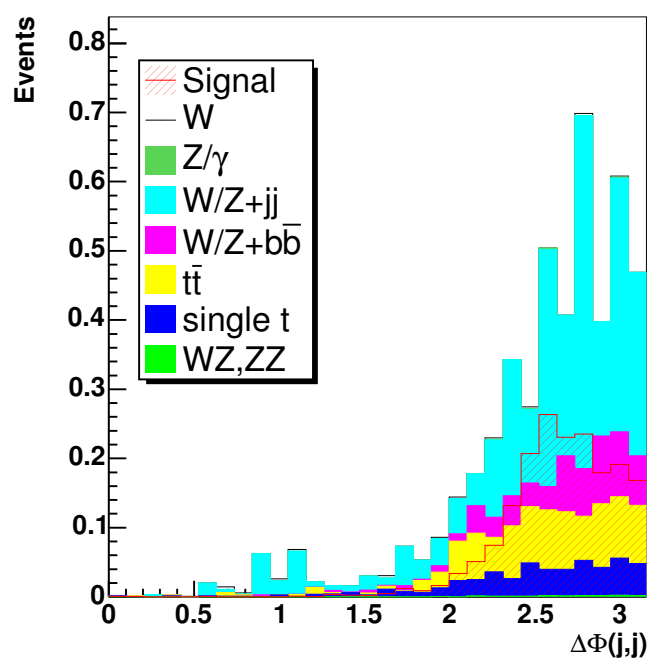

Figure C.15: Signal and background distributions of selection variables at $M_{\rho}=240 \mathrm{GeV} / c^{2}, M_{\pi}=140 \mathrm{GeV} / c^{2}$, and $M_{V}=100 \mathrm{GeV}$. 

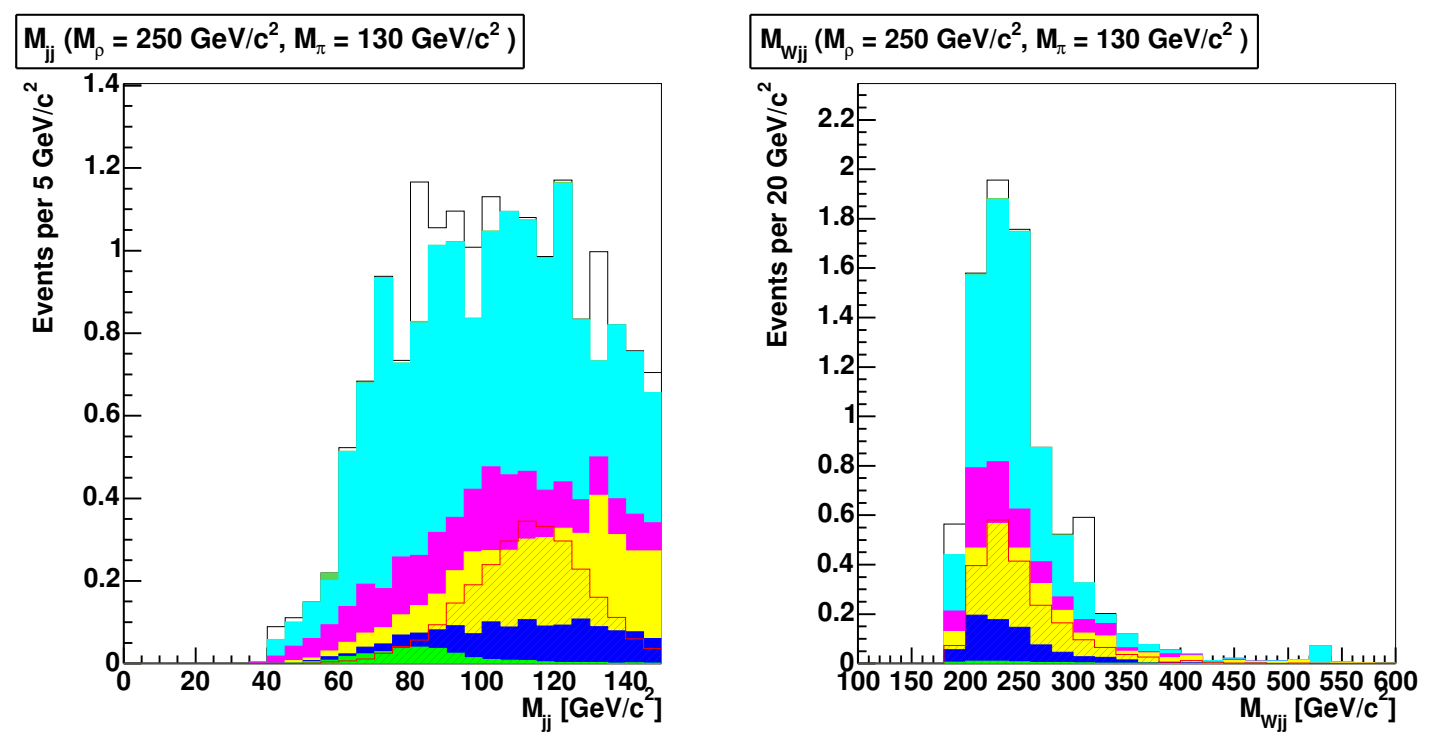

$\Delta \Phi(\mathrm{j}, \mathrm{j})\left(\mathrm{M}_{\mathrm{p}}=250 \mathrm{GeV} / \mathrm{c}^{2}, \mathrm{M}_{\pi}=130 \mathrm{GeV} / \mathrm{c}^{2}\right)$

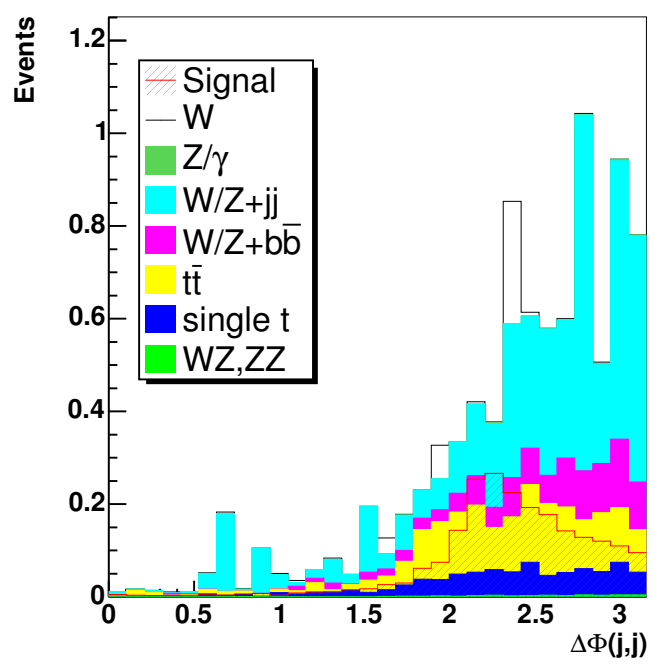

Figure C.16: Signal and background distributions of selection variables at $M_{\rho}=250 \mathrm{GeV} / c^{2}, M_{\pi}=130 \mathrm{GeV} / c^{2}$, and $M_{V}=100 \mathrm{GeV}$. 

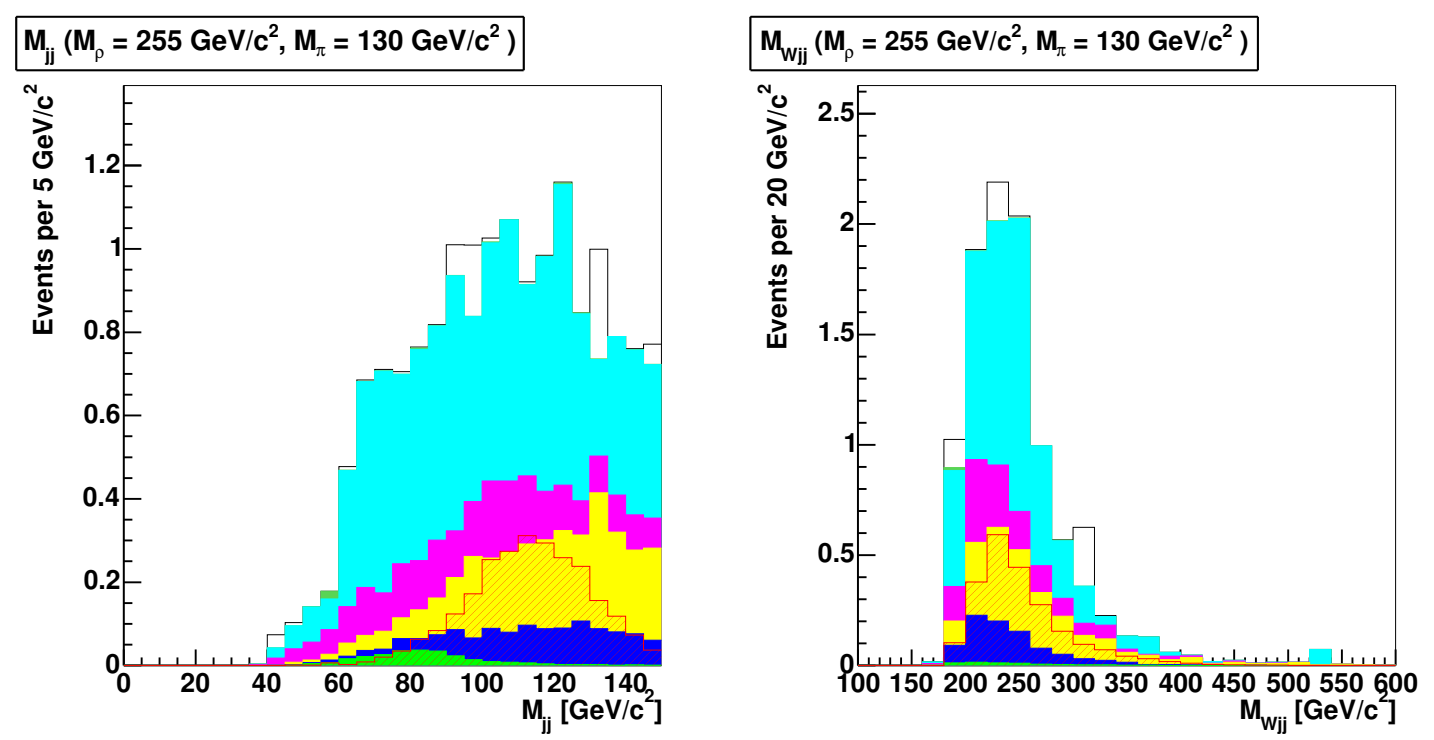

$\Delta \Phi(\mathrm{j}, \mathrm{j})\left(\mathrm{M}_{\mathrm{p}}=255 \mathrm{GeV} / \mathrm{c}^{2}, \mathrm{M}_{\pi}=130 \mathrm{GeV} / \mathrm{c}^{2}\right)$

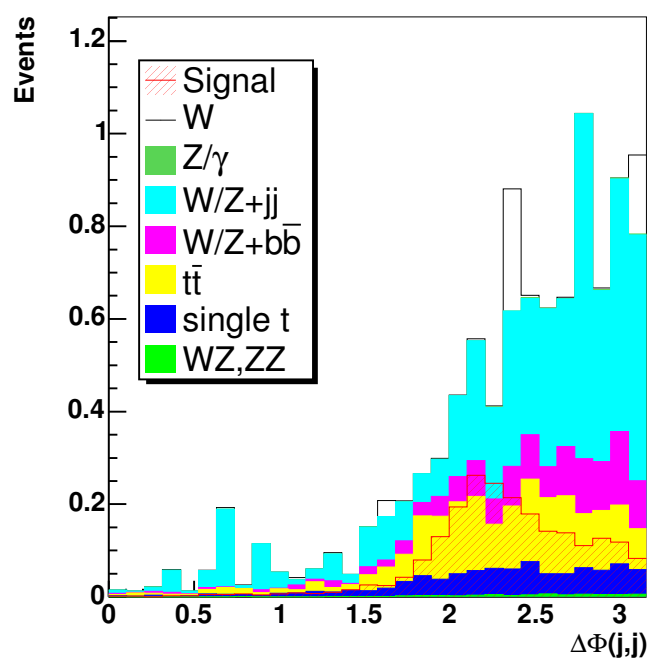

Figure C.17: Signal and background distributions of selection variables at $M_{\rho}=255 \mathrm{GeV} / c^{2}, M_{\pi}=130 \mathrm{GeV} / c^{2}$, and $M_{V}=100 \mathrm{GeV}$. 

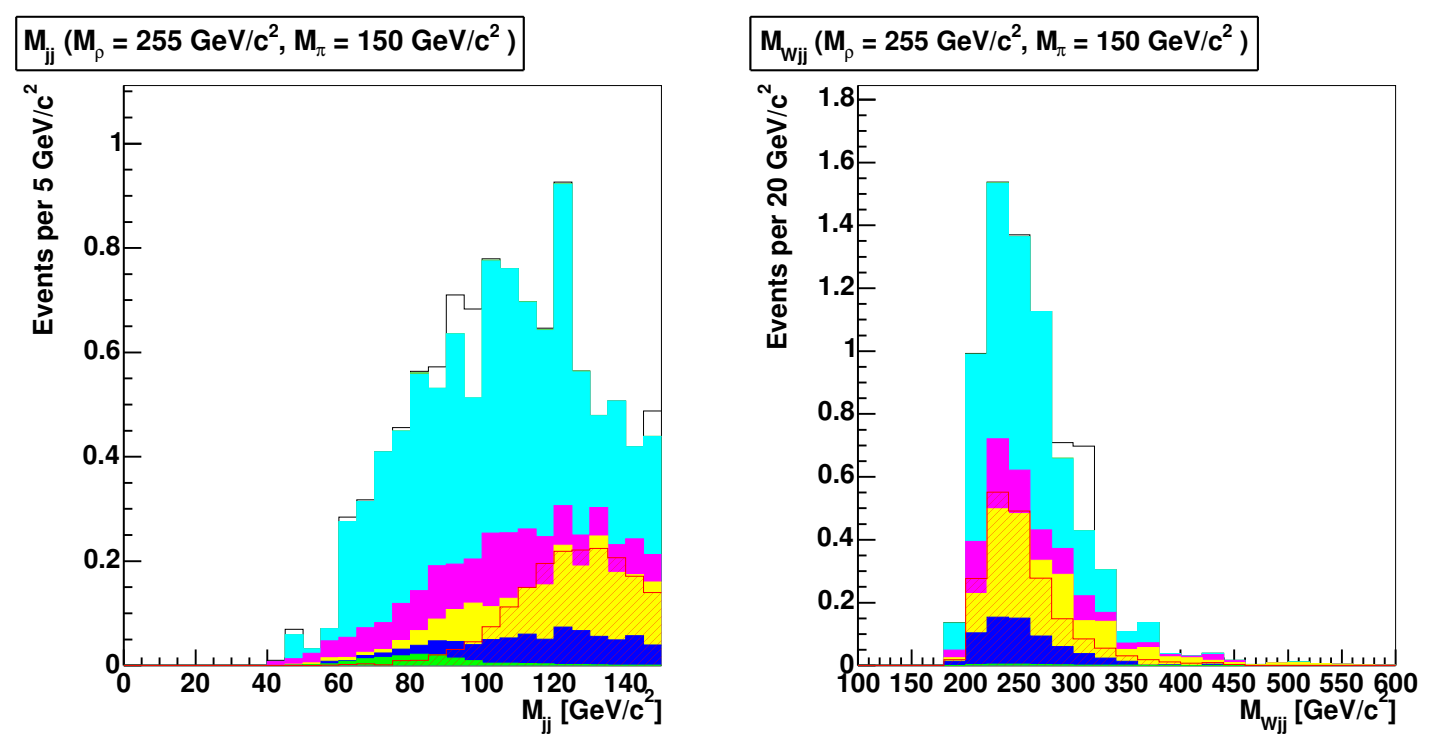

$\Delta \Phi(\mathrm{j}, \mathrm{j})\left(\mathrm{M}_{\mathrm{p}}=255 \mathrm{GeV} / \mathrm{c}^{2}, \mathrm{M}_{\pi}=150 \mathrm{GeV} / \mathrm{c}^{2}\right)$

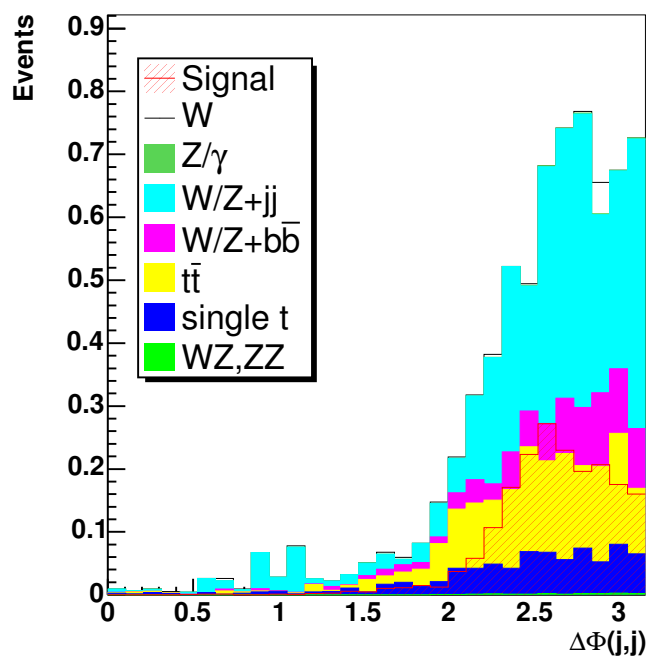

Figure C.18: Signal and background distributions of selection variables at $M_{\rho}=255 \mathrm{GeV} / c^{2}, M_{\pi}=150 \mathrm{GeV} / c^{2}$, and $M_{V}=100 \mathrm{GeV}$. 

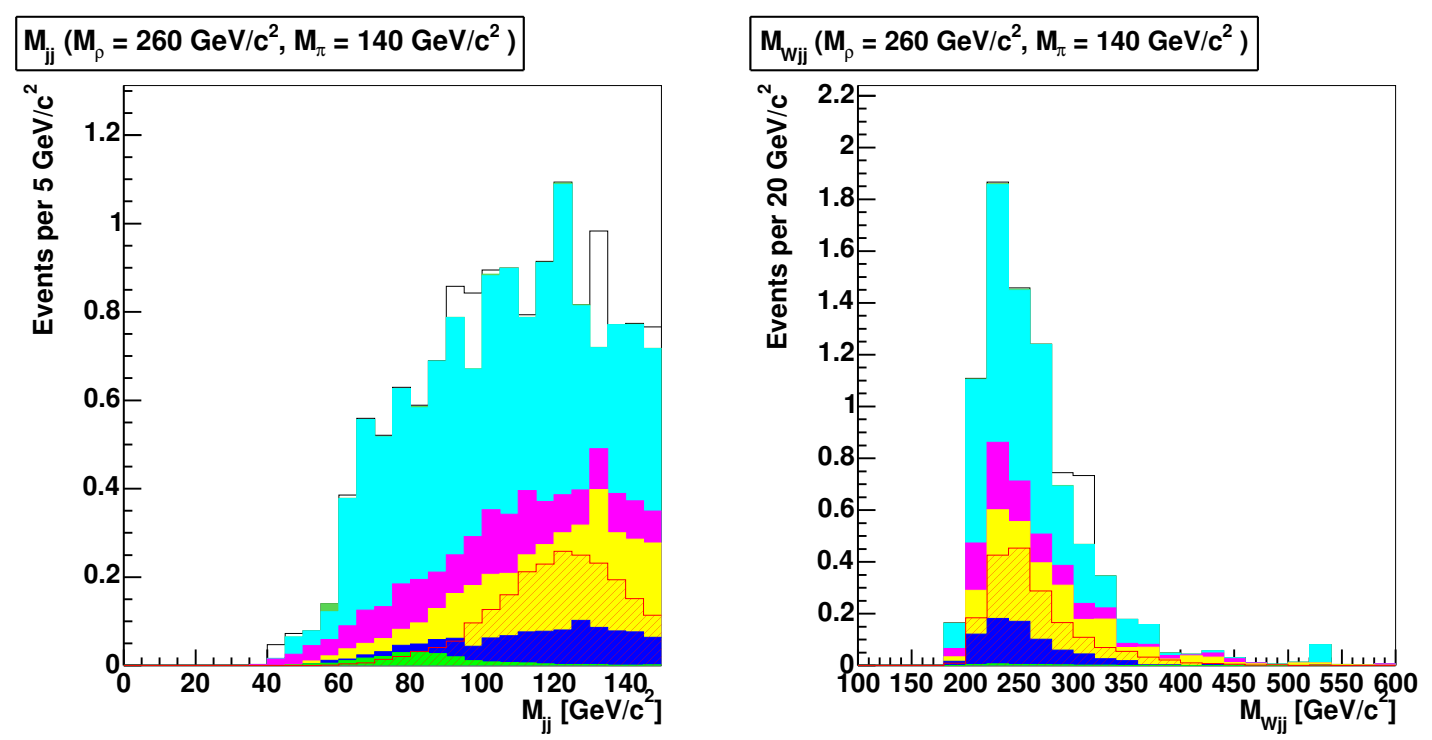

$\Delta \Phi(\mathrm{j}, \mathrm{j})\left(\mathrm{M}_{\mathrm{p}}=260 \mathrm{GeV} / \mathrm{c}^{2}, \mathrm{M}_{\pi}=140 \mathrm{GeV} / \mathrm{c}^{2}\right)$

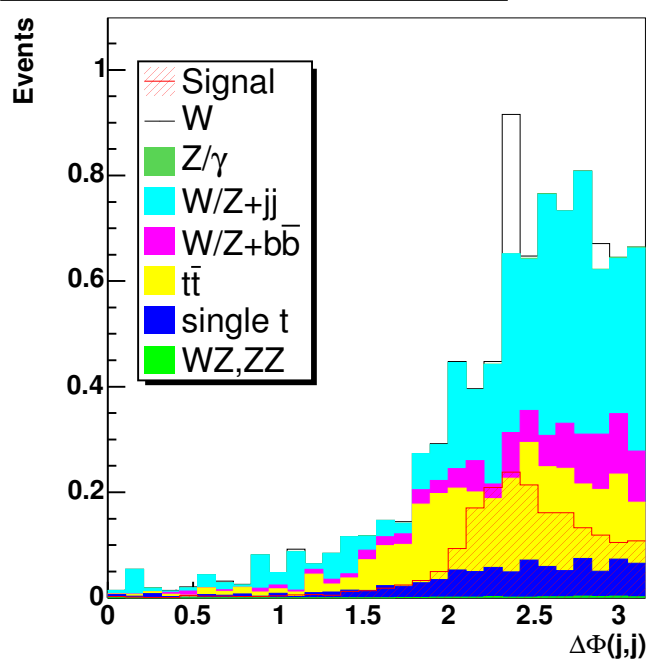

Figure C.19: Signal and background distributions of selection variables at $M_{\rho}=260 \mathrm{GeV} / c^{2}, M_{\pi}=140 \mathrm{GeV} / c^{2}$, and $M_{V}=100 \mathrm{GeV}$. 

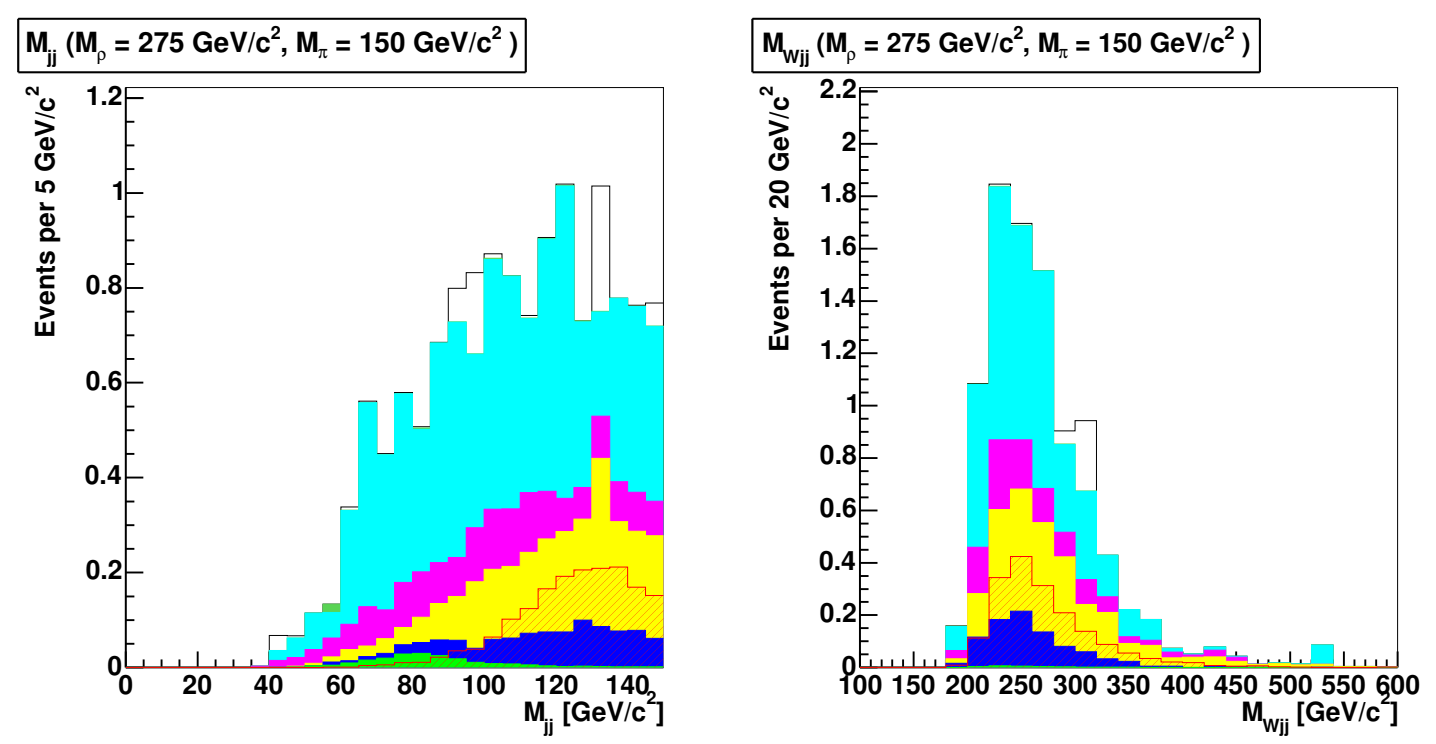

$\Delta \Phi(\mathrm{j}, \mathrm{j})\left(\mathrm{M}_{\mathrm{\rho}}=275 \mathrm{GeV} / \mathrm{c}^{2}, \mathrm{M}_{\pi}=150 \mathrm{GeV} / \mathrm{c}^{2}\right)$

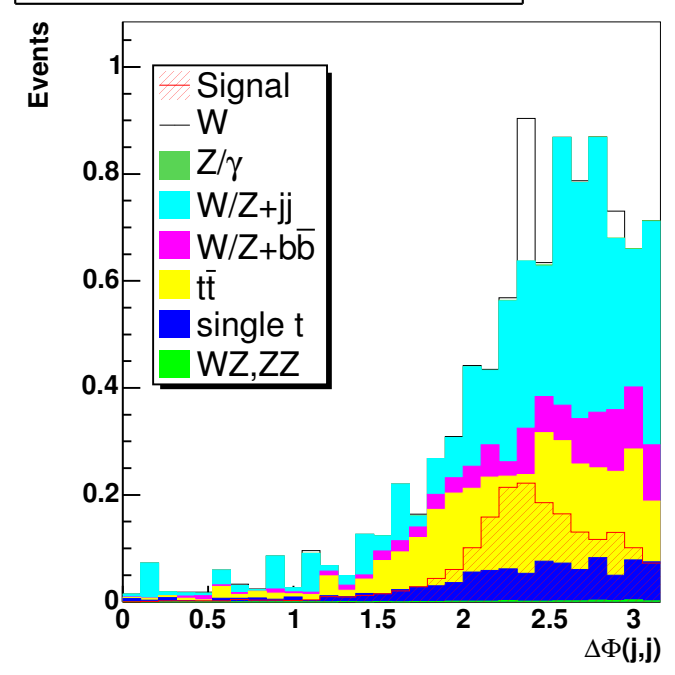

Figure C.20: Signal and background distributions of selection variables at $M_{\rho}=275 \mathrm{GeV} / c^{2}, M_{\pi}=150 \mathrm{GeV} / c^{2}$, and $M_{V}=100 \mathrm{GeV}$. 


\section{Appendix D}

\section{Systematic Uncertainties}

Systematic uncertainties are presented for all mass points as each selection requirement is imposed. 


\begin{tabular}{|l|lll|}
\hline sample & $m_{j j}$ & $m_{W j j}$ & $\Delta \phi(j, j)$ \\
\hline \hline Non- $W / Z$ BG Estimate & 2.2 & 0.92 & -0.010 \\
Cross sections & 1.7 & 0.46 & 0.30 \\
MC statistics & 0.32 & 0.20 & 0.16 \\
Diboson kinematics & 0.011 & 0.003 & 0.001 \\
Jet energy scale & 1.3 & 0.28 & 0.38 \\
b/c-jet TRF's & 0.83 & 0.20 & 0.12 \\
Mistag TRF's & -0.33 & -0.100 & -0.050 \\
Jet smearing & -1.1 & 0.050 & 0.31 \\
Track smearing & -1.5 & -0.020 & 0.14 \\
PV scale factor & -0.080 & 0.26 & 0.13 \\
Jet scale factor & -1.5 & 0.25 & 0.11 \\
Taggability scale factor & -1.2 & 0.35 & 0.20 \\
\hline Total BG prediction & 19 & 5.4 & 2.7 \\
Total BG error & 4.2 & 1.2 & 0.68 \\
\hline
\end{tabular}

Table D.1: Evolution of the systematic uncertainties on the backround with the final selection requirements, optimized for for $M_{\rho}=175 \mathrm{GeV} / c^{2}$, $M_{\pi}=90 \mathrm{GeV} / c^{2}$.

\begin{tabular}{|l|lll|}
\hline sample & $m_{j j}$ & $m_{W j j}$ & $\Delta \phi(j, j)$ \\
\hline \hline MC statistics & 0.11 & 0.086 & 0.080 \\
Jet energy scale & 0.57 & 0.25 & 0.20 \\
b/c-jet TRF's & 0.26 & 0.15 & 0.13 \\
Jet smearing & -0.72 & -0.75 & -0.66 \\
Track smearing & -0.31 & -0.35 & -0.31 \\
PV scale factor & 0.080 & -0.030 & -0.020 \\
Jet scale factor & -0.52 & -0.25 & -0.22 \\
Taggability scale factor & -0.40 & -0.18 & -0.13 \\
\hline Total signal prediction & 5.3 & 3.2 & 2.7 \\
Total signal error & 1.2 & 0.93 & 0.81 \\
Relative signal error & 0.23 & 0.29 & 0.30 \\
Signal acceptance & $3.9 \%$ & $2.4 \%$ & $2.0 \%$ \\
Acceptance error & $0.88 \%$ & $0.68 \%$ & $0.59 \%$ \\
\hline
\end{tabular}

Table D.2: Evolution of the systematic uncertainties on the signal with each selection requirement. The assumed masses are $M_{\rho}=175 \mathrm{GeV} / c^{2}$, $M_{\pi}=90 \mathrm{GeV} / c^{2}$, with $M_{V}=100 \mathrm{GeV}$. 


\begin{tabular}{|l|lll|}
\hline sample & $m_{j j}$ & $m_{W j j}$ & $\Delta \phi(j, j)$ \\
\hline \hline Non- $W / Z$ BG Estimate & 2.2 & 0.55 & -0.010 \\
Cross sections & 1.7 & 0.34 & 0.21 \\
MC statistics & 0.33 & 0.20 & 0.15 \\
Diboson kinematics & 0.010 & 0.001 & 0.001 \\
Jet energy scale & -0.81 & 0.46 & 0.22 \\
$b / c$-jet TRF's & 0.83 & 0.15 & 0.090 \\
Mistag TRF's & -0.33 & -0.070 & -0.040 \\
Jet smearing & -1.4 & 0.30 & 0.27 \\
Track smearing & -1.0 & 0.24 & 0.15 \\
PV scale factor & 0.84 & 1.00 & 0.47 \\
Jet scale factor & -1.7 & 0.75 & 0.41 \\
Taggability scale factor & -1.4 & 0.80 & 0.44 \\
\hline Total BG prediction & 19 & 3.9 & 1.9 \\
Total BG error & 4.2 & 1.7 & 0.90 \\
\hline
\end{tabular}

Table D.3: Evolution of the systematic uncertainties on the backround with the final selection requirements, optimized for for $M_{\rho}=180 \mathrm{GeV} / c^{2}$, $M_{\pi}=100 \mathrm{GeV} / c^{2}$.

\begin{tabular}{|l|lll|}
\hline sample & $m_{j j}$ & $m_{W j j}$ & $\Delta \phi(j, j)$ \\
\hline \hline MC statistics & 0.038 & 0.026 & 0.024 \\
Jet energy scale & -0.050 & 0.070 & 0.050 \\
b/c-jet TRF's & 0.090 & 0.040 & 0.030 \\
Jet smearing & -0.18 & -0.11 & -0.13 \\
Track smearing & 0 & -0.020 & -0.020 \\
PV scale factor & 0.080 & 0.040 & 0.030 \\
Jet scale factor & -0.14 & 0 & -0.010 \\
Taggability scale factor & -0.11 & 0.010 & 0 \\
\hline Total signal prediction & 1.8 & 0.82 & 0.67 \\
Total signal error & 0.29 & 0.15 & 0.15 \\
Relative signal error & 0.16 & 0.18 & 0.22 \\
Signal acceptance & $3.7 \%$ & $1.7 \%$ & $1.4 \%$ \\
Acceptance error & $0.58 \%$ & $0.30 \%$ & $0.30 \%$ \\
\hline
\end{tabular}

Table D.4: Evolution of the systematic uncertainties on the signal with each selection requirement. The assumed masses are $M_{\rho}=180 \mathrm{GeV} / c^{2}$, $M_{\pi}=100 \mathrm{GeV} / c^{2}$, with $M_{V}=100 \mathrm{GeV}$. 


\begin{tabular}{|l|lll|}
\hline sample & $m_{j j}$ & $m_{W j j}$ & $\Delta \phi(j, j)$ \\
\hline \hline Non- $W / Z$ BG Estimate & 2.2 & 0.55 & 0.050 \\
Cross sections & 1.7 & 0.37 & 0.28 \\
MC statistics & 0.32 & 0.20 & 0.16 \\
Diboson kinematics & 0.010 & 0.001 & 0.001 \\
Jet energy scale & -1.1 & 0.43 & 0.090 \\
$b / c$-jet TRF's & 0.86 & 0.17 & 0.12 \\
Mistag TRF's & -0.33 & -0.070 & -0.060 \\
Jet smearing & -1.7 & 0.22 & 0.34 \\
Track smearing & -1.4 & 0.080 & 0.17 \\
PV scale factor & 0.57 & 0.77 & 0.31 \\
Jet scale factor & -2.4 & 0.38 & 0.020 \\
Taggability scale factor & -2.1 & 0.43 & 0.080 \\
\hline Total BG prediction & 19 & 4.2 & 2.8 \\
Total BG error & 5.0 & 1.3 & 0.62 \\
\hline
\end{tabular}

Table D.5: Evolution of the systematic uncertainties on the backround with the final selection requirements, optimized for for $M_{\rho}=190 \mathrm{GeV} / c^{2}$, $M_{\pi}=100 \mathrm{GeV} / c^{2}$.

\begin{tabular}{|l|lll|}
\hline sample & $m_{j j}$ & $m_{W j j}$ & $\Delta \phi(j, j)$ \\
\hline \hline MC statistics & 0.090 & 0.062 & 0.061 \\
Jet energy scale & -0.23 & 0.18 & 0.17 \\
b/c-jet TRF's & 0.29 & 0.12 & 0.11 \\
Jet smearing & -0.59 & -0.42 & -0.38 \\
Track smearing & -0.13 & -0.100 & -0.080 \\
PV scale factor & 0.18 & 0.21 & 0.22 \\
Jet scale factor & -0.39 & -0.15 & -0.13 \\
Taggability scale factor & -0.30 & -0.060 & -0.050 \\
\hline Total signal prediction & 5.8 & 2.6 & 2.4 \\
Total signal error & 0.89 & 0.55 & 0.51 \\
Relative signal error & 0.15 & 0.22 & 0.21 \\
Signal acceptance & $4.6 \%$ & $2.0 \%$ & $2.0 \%$ \\
Acceptance error & $0.71 \%$ & $0.44 \%$ & $0.41 \%$ \\
\hline
\end{tabular}

Table D.6: Evolution of the systematic uncertainties on the signal with each selection requirement. The assumed masses are $M_{\rho}=190 \mathrm{GeV} / c^{2}$, $M_{\pi}=100 \mathrm{GeV} / c^{2}$, with $M_{V}=100 \mathrm{GeV}$. 


\begin{tabular}{|l|lll|}
\hline sample & $m_{j j}$ & $m_{W j j}$ & $\Delta \phi(j, j)$ \\
\hline \hline Non- $W / Z$ BG Estimate & 1.9 & 1.4 & 0.17 \\
Cross sections & 1.5 & 0.81 & 0.41 \\
MC statistics & 0.33 & 0.26 & 0.19 \\
Diboson kinematics & 0.007 & 0.003 & 0.001 \\
Jet energy scale & 0.59 & 0.31 & 0.010 \\
b/c-jet TRF's & 0.79 & 0.40 & 0.19 \\
Mistag TRF's & -0.30 & -0.15 & -0.080 \\
Jet smearing & -0.81 & -0.20 & -0.010 \\
Track smearing & -0.52 & -0.24 & 0.080 \\
PV scale factor & 0.23 & 0.88 & 0.51 \\
Jet scale factor & -0.92 & 0.22 & 0.31 \\
Taggability scale factor & -0.72 & 0.32 & 0.38 \\
\hline Total BG prediction & 17 & 9.4 & 4.1 \\
Total BG error & 3.1 & 2.0 & 0.89 \\
\hline
\end{tabular}

Table D.7: Evolution of the systematic uncertainties on the backround with the final selection requirements, optimized for for $M_{\rho}=190 \mathrm{GeV} / c^{2}$, $M_{\pi}=110 \mathrm{GeV} / c^{2}$.

\begin{tabular}{|l|lll|}
\hline sample & $m_{j j}$ & $m_{W j j}$ & $\Delta \phi(j, j)$ \\
\hline \hline MC statistics & 0.029 & 0.026 & 0.022 \\
Jet energy scale & 0.11 & 0.090 & 0.060 \\
$b / c$-jet TRF's & 0.080 & 0.060 & 0.040 \\
Jet smearing & -0.12 & -0.13 & -0.090 \\
Track smearing & -0.020 & -0.020 & -0.010 \\
PV scale factor & 0.030 & 0.030 & 0.020 \\
Jet scale factor & -0.070 & -0.040 & -0.010 \\
Taggability scale factor & -0.060 & -0.030 & -0.010 \\
\hline Total signal prediction & 1.5 & 1.1 & 0.83 \\
Total signal error & 0.21 & 0.18 & 0.12 \\
Relative signal error & 0.14 & 0.16 & 0.15 \\
Signal acceptance & $4.1 \%$ & $3.1 \%$ & $2.3 \%$ \\
Acceptance error & $0.58 \%$ & $0.50 \%$ & $0.33 \%$ \\
\hline
\end{tabular}

Table D.8: Evolution of the systematic uncertainties on the signal with each selection requirement. The assumed masses are $M_{\rho}=190 \mathrm{GeV} / c^{2}$, $M_{\pi}=110 \mathrm{GeV} / c^{2}$, with $M_{V}=100 \mathrm{GeV}$. 


\begin{tabular}{|l|lll|}
\hline sample & $m_{j j}$ & $m_{W j j}$ & $\Delta \phi(j, j)$ \\
\hline \hline Non- $W / Z$ BG Estimate & 2.2 & 1.9 & 0.61 \\
Cross sections & 1.7 & 1.1 & 0.71 \\
MC statistics & 0.33 & 0.28 & 0.22 \\
Diboson kinematics & 0.010 & 0.006 & 0.004 \\
Jet energy scale & 0.21 & 0.64 & 0.28 \\
$b / c$-jet TRF's & 0.84 & 0.52 & 0.33 \\
Mistag TRF's & -0.33 & -0.20 & -0.14 \\
Jet smearing & -1.6 & -0.82 & -0.24 \\
Track smearing & -1.2 & -0.67 & -0.36 \\
PV scale factor & 0.75 & 1.3 & 0.87 \\
Jet scale factor & -2.1 & -0.54 & -0.39 \\
Taggability scale factor & -1.9 & -0.39 & -0.26 \\
\hline Total BG prediction & 19 & 13 & 7.6 \\
Total BG error & 4.6 & 3.0 & 1.5 \\
\hline
\end{tabular}

Table D.9: Evolution of the systematic uncertainties on the backround with the final selection requirements, optimized for for $M_{\rho}=195 \mathrm{GeV} / c^{2}$, $M_{\pi}=100 \mathrm{GeV} / c^{2}$.

\begin{tabular}{|l|lll|}
\hline sample & $m_{j j}$ & $m_{W j j}$ & $\Delta \phi(j, j)$ \\
\hline \hline MC statistics & 0.097 & 0.089 & 0.084 \\
Jet energy scale & 0.38 & 0.31 & 0.24 \\
$b / c$-jet TRF's & 0.29 & 0.22 & 0.19 \\
Jet smearing & -0.64 & -0.62 & -0.55 \\
Track smearing & -0.100 & -0.21 & -0.17 \\
PV scale factor & 0.20 & 0.18 & 0.16 \\
Jet scale factor & -0.37 & -0.27 & -0.22 \\
Taggability scale factor & -0.28 & -0.19 & -0.15 \\
\hline Total signal prediction & 5.4 & 4.3 & 3.7 \\
Total signal error & 0.96 & 0.85 & 0.73 \\
Relative signal error & 0.18 & 0.20 & 0.19 \\
Signal acceptance & $4.5 \%$ & $3.6 \%$ & $3.1 \%$ \\
Acceptance error & $0.80 \%$ & $0.71 \%$ & $0.61 \%$ \\
\hline
\end{tabular}

Table D.10: Evolution of the systematic uncertainties on the signal with each selection requirement. The assumed masses are $M_{\rho}=195 \mathrm{GeV} / c^{2}$, $M_{\pi}=100 \mathrm{GeV} / c^{2}$, with $M_{V}=100 \mathrm{GeV}$. 


\begin{tabular}{|l|lll|}
\hline sample & $m_{j j}$ & $m_{W j j}$ & $\Delta \phi(j, j)$ \\
\hline \hline Non- $W / Z$ BG Estimate & 2.2 & 1.8 & 0.54 \\
Cross sections & 1.7 & 1.1 & 0.66 \\
MC statistics & 0.33 & 0.28 & 0.21 \\
Diboson kinematics & 0.010 & 0.005 & 0.004 \\
Jet energy scale & -0.98 & -0.18 & -0.22 \\
$b / c$-jet TRF's & 0.86 & 0.51 & 0.31 \\
Mistag TRF's & -0.34 & -0.20 & -0.13 \\
Jet smearing & -1.5 & -0.79 & -0.48 \\
Track smearing & -1.3 & -0.67 & -0.47 \\
PV scale factor & 0.66 & 1.3 & 0.63 \\
Jet scale factor & -2.0 & -0.55 & -0.51 \\
Taggability scale factor & -1.7 & -0.34 & -0.39 \\
\hline Total BG prediction & 19 & 12 & 7.1 \\
Total BG error & 4.6 & 2.8 & 1.5 \\
\hline
\end{tabular}

Table D.11: Evolution of the systematic uncertainties on the backround with the final selection requirements, optimized for for $M_{\rho}=200 \mathrm{GeV} / c^{2}$, $M_{\pi}=105 \mathrm{GeV} / c^{2}$.

\begin{tabular}{|l|lll|}
\hline sample & $m_{j j}$ & $m_{W j j}$ & $\Delta \phi(j, j)$ \\
\hline \hline MC statistics & 0.072 & 0.065 & 0.062 \\
Jet energy scale & 0.010 & 0 & 0.050 \\
$b / c$-jet TRF's & 0.29 & 0.22 & 0.20 \\
Jet smearing & -0.52 & -0.52 & -0.45 \\
Track smearing & -0.010 & -0.14 & -0.12 \\
PV scale factor & 0.23 & 0.21 & 0.21 \\
Jet scale factor & -0.21 & -0.18 & -0.16 \\
Taggability scale factor & -0.16 & -0.14 & -0.11 \\
\hline Total signal prediction & 5.4 & 4.3 & 3.8 \\
Total signal error & 0.69 & 0.66 & 0.59 \\
Relative signal error & 0.13 & 0.15 & 0.15 \\
Signal acceptance & $5.0 \%$ & $4.0 \%$ & $3.5 \%$ \\
Acceptance error & $0.64 \%$ & $0.61 \%$ & $0.54 \%$ \\
\hline
\end{tabular}

Table D.12: Evolution of the systematic uncertainties on the signal with each selection requirement. The assumed masses are $M_{\rho}=200 \mathrm{GeV} / c^{2}$, $M_{\pi}=105 \mathrm{GeV} / c^{2}$, with $M_{V}=100 \mathrm{GeV}$. 


\begin{tabular}{|l|lll|}
\hline sample & $m_{j j}$ & $m_{W j j}$ & $\Delta \phi(j, j)$ \\
\hline \hline Non- $W / Z$ BG Estimate & 1.3 & 0.74 & 0.19 \\
Cross sections & 1.0 & 0.24 & 0.17 \\
MC statistics & 0.29 & 0.14 & 0.13 \\
Diboson kinematics & 0.002 & 0 & 0 \\
Jet energy scale & -0.48 & 0.090 & 0.090 \\
b/c-jet TRF's & 0.59 & 0.11 & 0.080 \\
Mistag TRF's & -0.21 & -0.050 & -0.020 \\
Jet smearing & -0.71 & -0.21 & 0.11 \\
Track smearing & -0.79 & -0.28 & -0.17 \\
PV scale factor & 0.28 & 0.16 & 0.28 \\
Jet scale factor & -0.97 & -0.39 & 0.23 \\
Taggability scale factor & -0.76 & -0.32 & 0.29 \\
\hline Total BG prediction & 12 & 3.0 & 1.8 \\
Total BG error & 2.5 & 1.0 & 0.60 \\
\hline
\end{tabular}

Table D.13: Evolution of the systematic uncertainties on the backround with the final selection requirements, optimized for for $M_{\rho}=200 \mathrm{GeV} / c^{2}$, $M_{\pi}=120 \mathrm{GeV} / c^{2}$.

\begin{tabular}{|l|lll|}
\hline sample & $m_{j j}$ & $m_{W j j}$ & $\Delta \phi(j, j)$ \\
\hline \hline MC statistics & 0.021 & 0.015 & 0.014 \\
Jet energy scale & 0 & 0.040 & 0.030 \\
b/c-jet TRF's & 0.060 & 0.020 & 0.020 \\
Jet smearing & -0.11 & -0.080 & -0.080 \\
Track smearing & 0.030 & 0.010 & 0.010 \\
PV scale factor & 0.050 & 0.030 & 0.020 \\
Jet scale factor & -0.040 & -0.010 & -0.010 \\
Taggability scale factor & -0.030 & -0.010 & -0.010 \\
\hline Total signal prediction & 1.0 & 0.48 & 0.41 \\
Total signal error & 0.15 & 0.099 & 0.093 \\
Relative signal error & 0.15 & 0.21 & 0.23 \\
Signal acceptance & $3.9 \%$ & $1.9 \%$ & $1.6 \%$ \\
Acceptance error & $0.57 \%$ & $0.38 \%$ & $0.36 \%$ \\
\hline
\end{tabular}

Table D.14: Evolution of the systematic uncertainties on the signal with each selection requirement. The assumed masses are $M_{\rho}=200 \mathrm{GeV} / c^{2}$, $M_{\pi}=120 \mathrm{GeV} / c^{2}$, with $M_{V}=100 \mathrm{GeV}$. 


\begin{tabular}{|l|lll|}
\hline sample & $m_{j j}$ & $m_{W j j}$ & $\Delta \phi(j, j)$ \\
\hline \hline Non- $W / Z$ BG Estimate & 1.5 & 1.3 & 0.59 \\
Cross sections & 1.0 & 0.66 & 0.52 \\
MC statistics & 0.27 & 0.21 & 0.20 \\
Diboson kinematics & 0.004 & 0.002 & 0.001 \\
Jet energy scale & 0.28 & 0.23 & 0.17 \\
b/c-jet TRF's & 0.56 & 0.34 & 0.27 \\
Mistag TRF's & -0.19 & -0.15 & -0.11 \\
Jet smearing & -0.78 & -1.9 & -1.7 \\
Track smearing & -0.090 & -0.69 & -0.51 \\
PV scale factor & 0.33 & -0.17 & -0.30 \\
Jet scale factor & -0.33 & -0.24 & -0.54 \\
Taggability scale factor & -0.29 & -0.27 & -0.56 \\
\hline Total BG prediction & 12 & 8.1 & 5.9 \\
Total BG error & 2.1 & 2.6 & 2.2 \\
\hline
\end{tabular}

Table D.15: Evolution of the systematic uncertainties on the backround with the final selection requirements, optimized for for $M_{\rho}=210 \mathrm{GeV} / c^{2}$, $M_{\pi}=110 \mathrm{GeV} / c^{2}$.

\begin{tabular}{|l|lll|}
\hline sample & $m_{j j}$ & $m_{W j j}$ & $\Delta \phi(j, j)$ \\
\hline \hline MC statistics & 0.049 & 0.046 & 0.045 \\
Jet energy scale & 0.30 & 0.27 & 0.24 \\
b/c-jet TRF's & 0.19 & 0.16 & 0.16 \\
Jet smearing & -0.52 & -0.49 & -0.46 \\
Track smearing & -0.12 & -0.20 & -0.18 \\
PV scale factor & 0.030 & 0.050 & 0.050 \\
Jet scale factor & -0.20 & -0.14 & -0.12 \\
Taggability scale factor & -0.16 & -0.11 & -0.100 \\
\hline Total signal prediction & 3.3 & 2.9 & 2.6 \\
Total signal error & 0.69 & 0.64 & 0.60 \\
Relative signal error & 0.21 & 0.23 & 0.23 \\
Signal acceptance & $3.6 \%$ & $3.1 \%$ & $2.9 \%$ \\
Acceptance error & $0.75 \%$ & $0.69 \%$ & $0.64 \%$ \\
\hline
\end{tabular}

Table D.16: Evolution of the systematic uncertainties on the signal with each selection requirement. The assumed masses are $M_{\rho}=210 \mathrm{GeV} / c^{2}$, $M_{\pi}=110 \mathrm{GeV} / c^{2}$, with $M_{V}=100 \mathrm{GeV}$. 


\begin{tabular}{|l|lll|}
\hline sample & $m_{j j}$ & $m_{W j j}$ & $\Delta \phi(j, j)$ \\
\hline \hline Non- $W / Z$ BG Estimate & 1.9 & 1.1 & 0.25 \\
Cross sections & 1.3 & 0.48 & 0.27 \\
MC statistics & 0.34 & 0.18 & 0.14 \\
Diboson kinematics & 0.003 & 0.001 & 0 \\
Jet energy scale & -0.70 & -0.45 & -0.35 \\
b/c-jet TRF's & 0.82 & 0.25 & 0.13 \\
Mistag TRF's & -0.28 & -0.100 & -0.050 \\
Jet smearing & -1.4 & -0.51 & -0.51 \\
Track smearing & -0.91 & -0.40 & -0.43 \\
PV scale factor & 0.080 & 0.20 & 0.26 \\
Jet scale factor & -1.2 & -0.24 & -0.040 \\
Taggability scale factor & -0.88 & -0.14 & 0.020 \\
\hline Total BG prediction & 17 & 6.0 & 3.0 \\
Total BG error & 3.4 & 1.5 & 0.90 \\
\hline
\end{tabular}

Table D.17: Evolution of the systematic uncertainties on the backround with the final selection requirements, optimized for for $M_{\rho}=215 \mathrm{GeV} / c^{2}$, $M_{\pi}=130 \mathrm{GeV} / c^{2}$.

\begin{tabular}{|l|lll|}
\hline sample & $m_{j j}$ & $m_{W j j}$ & $\Delta \phi(j, j)$ \\
\hline \hline MC statistics & 0.042 & 0.033 & 0.032 \\
Jet energy scale & -0.020 & 0.090 & 0.060 \\
b/c-jet TRF's & 0.14 & 0.090 & 0.070 \\
Jet smearing & -0.14 & -0.21 & -0.21 \\
Track smearing & 0.010 & -0.030 & -0.050 \\
PV scale factor & 0.15 & 0.100 & 0.070 \\
Jet scale factor & -0.070 & -0.030 & -0.040 \\
Taggability scale factor & -0.070 & -0.030 & -0.040 \\
\hline Total signal prediction & 2.5 & 1.6 & 1.3 \\
Total signal error & 0.27 & 0.27 & 0.25 \\
Relative signal error & 0.11 & 0.18 & 0.19 \\
Signal acceptance & $5.8 \%$ & $3.6 \%$ & $3.1 \%$ \\
Acceptance error & $0.63 \%$ & $0.63 \%$ & $0.59 \%$ \\
\hline
\end{tabular}

Table D.18: Evolution of the systematic uncertainties on the signal with each selection requirement. The assumed masses are $M_{\rho}=215 \mathrm{GeV} / c^{2}$, $M_{\pi}=130 \mathrm{GeV} / c^{2}$, with $M_{V}=100 \mathrm{GeV}$. 


\begin{tabular}{|l|lll|}
\hline sample & $m_{j j}$ & $m_{W j j}$ & $\Delta \phi(j, j)$ \\
\hline \hline Non- $W / Z$ BG Estimate & 1.4 & 1.5 & 0.65 \\
Cross sections & 1.0 & 0.56 & 0.46 \\
MC statistics & 0.29 & 0.22 & 0.22 \\
Diboson kinematics & 0.002 & 0.001 & 0.001 \\
Jet energy scale & 0.12 & -0.070 & -0.100 \\
$b / c$-jet TRF's & 0.62 & 0.32 & 0.26 \\
Mistag TRF's & -0.22 & -0.12 & -0.100 \\
Jet smearing & -1.1 & -1.9 & -1.8 \\
Track smearing & -0.82 & -0.86 & -0.71 \\
PV scale factor & 0.100 & 0.37 & 0.24 \\
Jet scale factor & -1.1 & -0.45 & -0.55 \\
Taggability scale factor & -0.87 & -0.28 & -0.44 \\
\hline Total BG prediction & 13 & 7.5 & 5.6 \\
Total BG error & 2.7 & 2.7 & 2.3 \\
\hline
\end{tabular}

Table D.19: Evolution of the systematic uncertainties on the backround with the final selection requirements, optimized for for $M_{\rho}=220 \mathrm{GeV} / c^{2}$, $M_{\pi}=120 \mathrm{GeV} / c^{2}$.

\begin{tabular}{|l|lll|}
\hline sample & $m_{j j}$ & $m_{W j j}$ & $\Delta \phi(j, j)$ \\
\hline \hline MC statistics & 0.064 & 0.056 & 0.055 \\
Jet energy scale & 0.29 & 0.25 & 0.23 \\
b/c-jet TRF's & 0.21 & 0.15 & 0.14 \\
Jet smearing & -0.39 & -0.39 & -0.37 \\
Track smearing & 0.030 & -0.070 & -0.050 \\
PV scale factor & 0.15 & 0.100 & 0.080 \\
Jet scale factor & -0.12 & -0.050 & -0.070 \\
Taggability scale factor & -0.080 & -0.030 & -0.060 \\
\hline Total signal prediction & 3.5 & 2.5 & 2.4 \\
Total signal error & 0.57 & 0.51 & 0.48 \\
Relative signal error & 0.17 & 0.20 & 0.20 \\
Signal acceptance & $4.6 \%$ & $3.4 \%$ & $3.2 \%$ \\
Acceptance error & $0.77 \%$ & $0.68 \%$ & $0.64 \%$ \\
\hline
\end{tabular}

Table D.20: Evolution of the systematic uncertainties on the signal with each selection requirement. The assumed masses are $M_{\rho}=220 \mathrm{GeV} / c^{2}$, $M_{\pi}=120 \mathrm{GeV} / c^{2}$, with $M_{V}=100 \mathrm{GeV}$. 


\begin{tabular}{|l|lll|}
\hline sample & $m_{j j}$ & $m_{W j j}$ & $\Delta \phi(j, j)$ \\
\hline \hline Non- $W / Z$ BG Estimate & 1.4 & 1.1 & 0.22 \\
Cross sections & 1.1 & 0.53 & 0.30 \\
MC statistics & 0.32 & 0.23 & 0.16 \\
Diboson kinematics & 0.001 & 0 & 0 \\
Jet energy scale & 0.32 & 0.38 & 0.030 \\
b/c-jet TRF's & 0.70 & 0.31 & 0.16 \\
Mistag TRF's & -0.24 & -0.13 & -0.070 \\
Jet smearing & -1.2 & -1.4 & -0.90 \\
Track smearing & -0.49 & -0.40 & -0.090 \\
PV scale factor & -0.100 & -0.12 & 0.090 \\
Jet scale factor & -1.2 & -0.64 & -0.54 \\
Taggability scale factor & -1.0 & -0.46 & -0.42 \\
\hline Total BG prediction & 14 & 7.0 & 3.5 \\
Total BG error & 2.8 & 2.2 & 1.2 \\
\hline
\end{tabular}

Table D.21: Evolution of the systematic uncertainties on the backround with the final selection requirements, optimized for for $M_{\rho}=220 \mathrm{GeV} / c^{2}$, $M_{\pi}=140 \mathrm{GeV} / c^{2}$.

\begin{tabular}{|l|lll|}
\hline sample & $m_{j j}$ & $m_{W j j}$ & $\Delta \phi(j, j)$ \\
\hline \hline MC statistics & 0.014 & 0.012 & 0.011 \\
Jet energy scale & 0.050 & 0.030 & 0.040 \\
b/c-jet TRF's & 0.050 & 0.030 & 0.030 \\
Jet smearing & -0.050 & -0.070 & -0.050 \\
Track smearing & 0.010 & 0 & 0.010 \\
PV scale factor & 0.040 & 0.040 & 0.040 \\
Jet scale factor & -0.010 & -0.010 & 0.010 \\
Taggability scale factor & -0.020 & -0.010 & 0.010 \\
\hline Total signal prediction & 0.77 & 0.57 & 0.48 \\
Total signal error & 0.099 & 0.093 & 0.084 \\
Relative signal error & 0.13 & 0.16 & 0.17 \\
Signal acceptance & $5.0 \%$ & $3.7 \%$ & $3.1 \%$ \\
Acceptance error & $0.65 \%$ & $0.61 \%$ & $0.54 \%$ \\
\hline
\end{tabular}

Table D.22: Evolution of the systematic uncertainties on the signal with each selection requirement. The assumed masses are $M_{\rho}=220 \mathrm{GeV} / c^{2}$, $M_{\pi}=140 \mathrm{GeV} / c^{2}$, with $M_{V}=100 \mathrm{GeV}$. 


\begin{tabular}{|l|lll|}
\hline sample & $m_{j j}$ & $m_{W j j}$ & $\Delta \phi(j, j)$ \\
\hline \hline Non- $W / Z$ BG Estimate & 1.7 & 1.3 & 0.88 \\
Cross sections & 1.3 & 1.1 & 0.93 \\
MC statistics & 0.33 & 0.29 & 0.30 \\
Diboson kinematics & 0.005 & 0.004 & 0.003 \\
Jet energy scale & -0.98 & -0.47 & -0.26 \\
$b / c$-jet TRF's & 0.74 & 0.63 & 0.54 \\
Mistag TRF's & -0.26 & -0.21 & -0.18 \\
Jet smearing & -0.46 & -0.52 & -0.49 \\
Track smearing & -0.15 & -0.88 & -0.66 \\
PV scale factor & 0.29 & -0.22 & 0 \\
Jet scale factor & -0.79 & -0.68 & -0.32 \\
Taggability scale factor & -0.51 & -0.54 & -0.23 \\
\hline Total BG prediction & 15 & 13 & 11 \\
Total BG error & 2.7 & 2.3 & 1.7 \\
\hline
\end{tabular}

Table D.23: Evolution of the systematic uncertainties on the backround with the final selection requirements, optimized for for $M_{\rho}=235 \mathrm{GeV} / c^{2}$, $M_{\pi}=120 \mathrm{GeV} / c^{2}$.

\begin{tabular}{|l|lll|}
\hline sample & $m_{j j}$ & $m_{W j j}$ & $\Delta \phi(j, j)$ \\
\hline \hline MC statistics & 0.055 & 0.053 & 0.052 \\
Jet energy scale & 0.090 & 0.040 & 0.060 \\
$b / c$-jet TRF's & 0.18 & 0.18 & 0.16 \\
Jet smearing & -0.31 & -0.31 & -0.28 \\
Track smearing & 0.010 & -0.030 & -0.010 \\
PV scale factor & 0.13 & 0.13 & 0.14 \\
Jet scale factor & -0.100 & -0.050 & -0.050 \\
Taggability scale factor & -0.070 & -0.040 & -0.040 \\
\hline Total signal prediction & 3.1 & 2.9 & 2.6 \\
Total signal error & 0.41 & 0.39 & 0.37 \\
Relative signal error & 0.13 & 0.14 & 0.14 \\
Signal acceptance & $4.9 \%$ & $4.5 \%$ & $4.2 \%$ \\
Acceptance error & $0.66 \%$ & $0.62 \%$ & $0.58 \%$ \\
\hline
\end{tabular}

Table D.24: Evolution of the systematic uncertainties on the signal with each selection requirement. The assumed masses are $M_{\rho}=235 \mathrm{GeV} / c^{2}$, $M_{\pi}=120 \mathrm{GeV} / c^{2}$, with $M_{V}=100 \mathrm{GeV}$. 


\begin{tabular}{|l|lll|}
\hline sample & $m_{j j}$ & $m_{W j j}$ & $\Delta \phi(j, j)$ \\
\hline \hline Non- $W / Z$ BG Estimate & 1.1 & 1.1 & 0.56 \\
Cross sections & 0.98 & 0.57 & 0.51 \\
MC statistics & 0.32 & 0.23 & 0.24 \\
Diboson kinematics & 0.001 & 0.001 & 0.001 \\
Jet energy scale & 0.24 & 0.080 & 0.13 \\
b/c-jet TRF's & 0.64 & 0.34 & 0.31 \\
Mistag TRF's & -0.22 & -0.14 & -0.11 \\
Jet smearing & -0.77 & -1.6 & -1.4 \\
Track smearing & -0.88 & -1.1 & -1.1 \\
PV scale factor & -0.080 & -0.050 & 0.21 \\
Jet scale factor & -1.6 & -0.98 & -0.95 \\
Taggability scale factor & -1.2 & -0.81 & -0.81 \\
\hline Total BG prediction & 12 & 7.5 & 6.2 \\
Total BG error & 2.9 & 2.7 & 2.3 \\
\hline
\end{tabular}

Table D.25: Evolution of the systematic uncertainties on the backround with the final selection requirements, optimized for for $M_{\rho}=235 \mathrm{GeV} / c^{2}$, $M_{\pi}=130 \mathrm{GeV} / c^{2}$.

\begin{tabular}{|l|lll|}
\hline sample & $m_{j j}$ & $m_{W j j}$ & $\Delta \phi(j, j)$ \\
\hline \hline MC statistics & 0.053 & 0.045 & 0.045 \\
Jet energy scale & 0.19 & 0.13 & 0.11 \\
b/c-jet TRF's & 0.19 & 0.13 & 0.12 \\
Jet smearing & -0.35 & -0.42 & -0.41 \\
Track smearing & -0.020 & -0.12 & -0.13 \\
PV scale factor & 0.14 & 0.090 & 0.070 \\
Jet scale factor & -0.13 & -0.12 & -0.13 \\
Taggability scale factor & -0.14 & -0.13 & -0.14 \\
\hline Total signal prediction & 3.0 & 2.1 & 2.0 \\
Total signal error & 0.50 & 0.52 & 0.50 \\
Relative signal error & 0.17 & 0.25 & 0.25 \\
Signal acceptance & $5.1 \%$ & $3.6 \%$ & $3.4 \%$ \\
Acceptance error & $0.86 \%$ & $0.88 \%$ & $0.86 \%$ \\
\hline
\end{tabular}

Table D.26: Evolution of the systematic uncertainties on the signal with each selection requirement. The assumed masses are $M_{\rho}=235 \mathrm{GeV} / c^{2}$, $M_{\pi}=130 \mathrm{GeV} / c^{2}$, with $M_{V}=100 \mathrm{GeV}$. 


\begin{tabular}{|l|lll|}
\hline sample & $m_{j j}$ & $m_{W j j}$ & $\Delta \phi(j, j)$ \\
\hline \hline Non- $W / Z$ BG Estimate & 1.0 & 0.76 & 0.100 \\
Cross sections & 0.60 & 0.40 & 0.21 \\
MC statistics & 0.26 & 0.19 & 0.13 \\
Diboson kinematics & 0 & 0 & 0 \\
Jet energy scale & -0.63 & -0.33 & -0.23 \\
$b / c$-jet TRF's & 0.47 & 0.27 & 0.13 \\
Mistag TRF's & -0.16 & -0.100 & -0.050 \\
Jet smearing & -1.1 & -1.1 & -0.48 \\
Track smearing & -0.61 & -0.13 & 0.100 \\
PV scale factor & -0.71 & -0.68 & -0.15 \\
Jet scale factor & -1.2 & -0.96 & -0.58 \\
Taggability scale factor & -1.1 & -0.96 & -0.44 \\
\hline Total BG prediction & 8.9 & 5.5 & 2.5 \\
Total BG error & 2.7 & 2.1 & 0.97 \\
\hline
\end{tabular}

Table D.27: Evolution of the systematic uncertainties on the backround with the final selection requirements, optimized for for $M_{\rho}=235 \mathrm{GeV} / c^{2}$, $M_{\pi}=150 \mathrm{GeV} / c^{2}$.

\begin{tabular}{|l|lll|}
\hline sample & $m_{j j}$ & $m_{W j j}$ & $\Delta \phi(j, j)$ \\
\hline \hline MC statistics & 0.025 & 0.024 & 0.022 \\
Jet energy scale & -0.020 & 0 & 0.010 \\
$b / c$-jet TRF's & 0.080 & 0.070 & 0.060 \\
Jet smearing & -0.15 & -0.15 & -0.12 \\
Track smearing & 0.020 & -0.010 & -0.010 \\
PV scale factor & 0.050 & 0.030 & 0.030 \\
Jet scale factor & -0.040 & -0.040 & -0.030 \\
Taggability scale factor & -0.060 & -0.060 & -0.050 \\
\hline Total signal prediction & 1.3 & 1.1 & 0.96 \\
Total signal error & 0.20 & 0.18 & 0.15 \\
Relative signal error & 0.15 & 0.16 & 0.16 \\
Signal acceptance & $4.8 \%$ & $4.2 \%$ & $3.5 \%$ \\
Acceptance error & $0.71 \%$ & $0.67 \%$ & $0.55 \%$ \\
\hline
\end{tabular}

Table D.28: Evolution of the systematic uncertainties on the signal with each selection requirement. The assumed masses are $M_{\rho}=235 \mathrm{GeV} / c^{2}$, $M_{\pi}=150 \mathrm{GeV} / c^{2}$, with $M_{V}=100 \mathrm{GeV}$. 


\begin{tabular}{|l|lll|}
\hline sample & $m_{j j}$ & $m_{W j j}$ & $\Delta \phi(j, j)$ \\
\hline \hline Non- $W / Z$ BG Estimate & 0.79 & 0.67 & 0.14 \\
Cross sections & 0.74 & 0.49 & 0.42 \\
MC statistics & 0.29 & 0.22 & 0.23 \\
Diboson kinematics & 0.001 & 0 & 0 \\
Jet energy scale & 0.060 & -0.11 & 0.090 \\
$b / c$-jet TRF's & -0.14 & 0.030 & 0.22 \\
Mistag TRF's & -0.82 & -0.39 & -0.12 \\
Jet smearing & -1.3 & -1.6 & -1.00 \\
Track smearing & -0.72 & -0.53 & -0.050 \\
PV scale factor & -0.66 & -0.45 & -0.23 \\
Jet scale factor & -0.94 & -0.58 & -0.090 \\
Taggability scale factor & -0.65 & -0.45 & -0.050 \\
\hline Total BG prediction & 9.1 & 6.0 & 4.6 \\
Total BG error & 2.4 & 2.1 & 1.2 \\
\hline
\end{tabular}

Table D.29: Evolution of the systematic uncertainties on the backround with the final selection requirements, optimized for for $M_{\rho}=240 \mathrm{GeV} / c^{2}$, $M_{\pi}=140 \mathrm{GeV} / c^{2}$.

\begin{tabular}{|l|lll|}
\hline sample & $m_{j j}$ & $m_{W j j}$ & $\Delta \phi(j, j)$ \\
\hline \hline MC statistics & 0.042 & 0.039 & 0.038 \\
Jet energy scale & 0.100 & 0.090 & 0.090 \\
$b / c$-jet TRF's & 0.14 & 0.11 & 0.11 \\
Jet smearing & -0.27 & -0.24 & -0.22 \\
Track smearing & -0.030 & -0.070 & -0.080 \\
PV scale factor & 0.14 & 0.17 & 0.15 \\
Jet scale factor & -0.060 & 0 & 0 \\
Taggability scale factor & -0.060 & -0.010 & -0.010 \\
\hline Total signal prediction & 2.2 & 1.8 & 1.7 \\
Total signal error & 0.36 & 0.34 & 0.31 \\
Relative signal error & 0.16 & 0.18 & 0.19 \\
Signal acceptance & $4.5 \%$ & $3.7 \%$ & $3.4 \%$ \\
Acceptance error & $0.74 \%$ & $0.69 \%$ & $0.64 \%$ \\
\hline
\end{tabular}

Table D.30: Evolution of the systematic uncertainties on the signal with each selection requirement. The assumed masses are $M_{\rho}=240 \mathrm{GeV} / c^{2}$, $M_{\pi}=140 \mathrm{GeV} / c^{2}$, with $M_{V}=100 \mathrm{GeV}$. 


\begin{tabular}{|l|lll|}
\hline sample & $m_{j j}$ & $m_{W j j}$ & $\Delta \phi(j, j)$ \\
\hline \hline Non- $W / Z$ BG Estimate & 1.0 & 0.85 & 0.54 \\
Cross sections & 0.91 & 0.83 & 0.73 \\
MC statistics & 0.31 & 0.31 & 0.32 \\
Diboson kinematics & 0.001 & 0.001 & 0.001 \\
Jet energy scale & 0.060 & 0.20 & 0.28 \\
$b / c$-jet TRF's & 0.58 & 0.53 & 0.47 \\
Mistag TRF's & -0.19 & -0.17 & -0.15 \\
Jet smearing & -0.49 & -0.090 & -0.070 \\
Track smearing & -0.57 & -0.040 & 0.17 \\
PV scale factor & 0.37 & 0.51 & 0.89 \\
Jet scale factor & -1.3 & -0.68 & -0.30 \\
Taggability scale factor & -0.95 & -0.40 & -0.15 \\
\hline Total BG prediction & 11 & 10 & 8.8 \\
Total BG error & 2.4 & 1.7 & 1.5 \\
\hline
\end{tabular}

Table D.31: Evolution of the systematic uncertainties on the backround with the final selection requirements, optimized for for $M_{\rho}=250 \mathrm{GeV} / c^{2}$, $M_{\pi}=130 \mathrm{GeV} / c^{2}$.

\begin{tabular}{|l|lll|}
\hline sample & $m_{j j}$ & $m_{W j j}$ & $\Delta \phi(j, j)$ \\
\hline \hline MC statistics & 0.032 & 0.031 & 0.030 \\
Jet energy scale & 0.13 & 0.14 & 0.11 \\
b/c-jet TRF's & 0.14 & 0.14 & 0.13 \\
Jet smearing & -0.28 & -0.28 & -0.28 \\
Track smearing & -0.010 & -0.040 & -0.040 \\
PV scale factor & 0.100 & 0.11 & 0.100 \\
Jet scale factor & -0.050 & -0.030 & -0.040 \\
Taggability scale factor & -0.070 & -0.050 & -0.060 \\
\hline Total signal prediction & 2.2 & 2.1 & 1.9 \\
Total signal error & 0.37 & 0.37 & 0.35 \\
Relative signal error & 0.16 & 0.17 & 0.18 \\
Signal acceptance & $4.6 \%$ & $4.3 \%$ & $4.0 \%$ \\
Acceptance error & $0.75 \%$ & $0.76 \%$ & $0.73 \%$ \\
\hline
\end{tabular}

Table D.32: Evolution of the systematic uncertainties on the signal with each selection requirement. The assumed masses are $M_{\rho}=250 \mathrm{GeV} / c^{2}$, $M_{\pi}=130 \mathrm{GeV} / c^{2}$, with $M_{V}=100 \mathrm{GeV}$. 


\begin{tabular}{|l|lll|}
\hline sample & $m_{j j}$ & $m_{W j j}$ & $\Delta \phi(j, j)$ \\
\hline \hline Non- $W / Z$ BG Estimate & 1.7 & 0.96 & 0.54 \\
Cross sections & 1.1 & 0.94 & 0.81 \\
MC statistics & 0.33 & 0.31 & 0.32 \\
Diboson kinematics & 0.001 & 0.001 & 0.001 \\
Jet energy scale & 0.14 & 0.20 & 0.20 \\
$b / c$-jet TRF's & 0.72 & 0.62 & 0.55 \\
Mistag TRF's & -0.24 & -0.20 & -0.17 \\
Jet smearing & -0.32 & -0.27 & -0.53 \\
Track smearing & -0.64 & -0.36 & -0.38 \\
PV scale factor & 0.32 & 0.15 & 0.16 \\
Jet scale factor & -1.2 & -0.38 & -0.34 \\
Taggability scale factor & -0.83 & -0.11 & -0.19 \\
\hline Total BG prediction & 14 & 12 & 10.0 \\
Total BG error & 2.8 & 1.7 & 1.4 \\
\hline
\end{tabular}

Table D.33: Evolution of the systematic uncertainties on the backround with the final selection requirements, optimized for for $M_{\rho}=255 \mathrm{GeV} / c^{2}$, $M_{\pi}=130 \mathrm{GeV} / c^{2}$.

\begin{tabular}{|l|lll|}
\hline sample & $m_{j j}$ & $m_{W j j}$ & $\Delta \phi(j, j)$ \\
\hline \hline MC statistics & 0.043 & 0.041 & 0.041 \\
Jet energy scale & 0.080 & 0.090 & 0.080 \\
b/c-jet TRF's & 0.16 & 0.14 & 0.14 \\
Jet smearing & -0.28 & -0.26 & -0.23 \\
Track smearing & 0.010 & -0.040 & -0.010 \\
PV scale factor & 0.11 & 0.090 & 0.090 \\
Jet scale factor & -0.030 & -0.040 & -0.020 \\
Taggability scale factor & -0.040 & -0.060 & -0.040 \\
\hline Total signal prediction & 2.4 & 2.2 & 2.1 \\
Total signal error & 0.36 & 0.33 & 0.30 \\
Relative signal error & 0.15 & 0.15 & 0.15 \\
Signal acceptance & $5.2 \%$ & $4.8 \%$ & $4.4 \%$ \\
Acceptance error & $0.76 \%$ & $0.72 \%$ & $0.65 \%$ \\
\hline
\end{tabular}

Table D.34: Evolution of the systematic uncertainties on the signal with each selection requirement. The assumed masses are $M_{\rho}=255 \mathrm{GeV} / c^{2}$, $M_{\pi}=130 \mathrm{GeV} / c^{2}$, with $M_{V}=100 \mathrm{GeV}$. 


\begin{tabular}{|l|lll|}
\hline sample & $m_{j j}$ & $m_{W j j}$ & $\Delta \phi(j, j)$ \\
\hline \hline Non- $W / Z$ BG Estimate & 1.1 & 0.91 & 0.26 \\
Cross sections & 0.87 & 0.69 & 0.57 \\
MC statistics & 0.29 & 0.24 & 0.24 \\
Diboson kinematics & 0.001 & 0 & 0 \\
Jet energy scale & -0.31 & -0.38 & -0.040 \\
b/c-jet TRF's & 0.60 & 0.44 & 0.36 \\
Mistag TRF's & -0.21 & -0.18 & -0.13 \\
Jet smearing & -0.91 & -1.3 & -0.85 \\
Track smearing & -0.33 & -0.58 & -0.25 \\
PV scale factor & -0.32 & -0.40 & -0.070 \\
Jet scale factor & -0.85 & -0.86 & -0.25 \\
Taggability scale factor & -0.69 & -0.83 & -0.39 \\
\hline Total BG prediction & 11 & 8.9 & 6.7 \\
Total BG error & 2.2 & 2.3 & 1.3 \\
\hline
\end{tabular}

Table D.35: Evolution of the systematic uncertainties on the backround with the final selection requirements, optimized for for $M_{\rho}=255 \mathrm{GeV} / c^{2}$, $M_{\pi}=150 \mathrm{GeV} / c^{2}$.

\begin{tabular}{|l|lll|}
\hline sample & $m_{j j}$ & $m_{W j j}$ & $\Delta \phi(j, j)$ \\
\hline \hline MC statistics & 0.038 & 0.036 & 0.035 \\
Jet energy scale & 0.040 & 0.020 & 0.030 \\
b/c-jet TRF's & 0.15 & 0.13 & 0.12 \\
Jet smearing & -0.21 & -0.23 & -0.23 \\
Track smearing & 0.030 & -0.030 & -0.030 \\
PV scale factor & 0.070 & 0.070 & 0.050 \\
Jet scale factor & 0.020 & 0.030 & 0.020 \\
Taggability scale factor & 0.010 & 0.030 & 0.010 \\
\hline Total signal prediction & 2.2 & 2.0 & 1.8 \\
Total signal error & 0.28 & 0.28 & 0.27 \\
Relative signal error & 0.12 & 0.14 & 0.15 \\
Signal acceptance & $5.7 \%$ & $5.1 \%$ & $4.6 \%$ \\
Acceptance error & $0.70 \%$ & $0.72 \%$ & $0.69 \%$ \\
\hline
\end{tabular}

Table D.36: Evolution of the systematic uncertainties on the signal with each selection requirement. The assumed masses are $M_{\rho}=255 \mathrm{GeV} / c^{2}$, $M_{\pi}=150 \mathrm{GeV} / c^{2}$, with $M_{V}=100 \mathrm{GeV}$. 


\begin{tabular}{|l|lll|}
\hline sample & $m_{j j}$ & $m_{W j j}$ & $\Delta \phi(j, j)$ \\
\hline \hline Non- $W / Z$ BG Estimate & 0.91 & 0.79 & 0.36 \\
Cross sections & 0.83 & 0.71 & 0.61 \\
MC statistics & 0.30 & 0.28 & 0.29 \\
Diboson kinematics & 0.001 & 0 & 0 \\
Jet energy scale & 0.71 & 0.57 & 0.62 \\
$b / c$-jet TRF's & 0.56 & 0.48 & 0.44 \\
Mistag TRF's & -0.19 & -0.18 & -0.15 \\
Jet smearing & -0.50 & -0.71 & -0.55 \\
Track smearing & -0.090 & -0.46 & -0.48 \\
PV scale factor & -0.37 & -0.60 & -0.66 \\
Jet scale factor & -0.54 & -0.67 & -0.44 \\
Taggability scale factor & -0.43 & -0.57 & -0.41 \\
\hline Total BG prediction & 11 & 9.2 & 7.9 \\
Total BG error & 1.8 & 1.9 & 1.6 \\
\hline
\end{tabular}

Table D.37: Evolution of the systematic uncertainties on the backround with the final selection requirements, optimized for for $M_{\rho}=260 \mathrm{GeV} / c^{2}$, $M_{\pi}=140 \mathrm{GeV} / c^{2}$.

\begin{tabular}{|l|lll|}
\hline sample & $m_{j j}$ & $m_{W j j}$ & $\Delta \phi(j, j)$ \\
\hline \hline MC statistics & 0.037 & 0.036 & 0.035 \\
Jet energy scale & 0.090 & 0.11 & 0.100 \\
b/c-jet TRF's & 0.13 & 0.12 & 0.12 \\
Jet smearing & -0.26 & -0.24 & -0.22 \\
Track smearing & -0.080 & -0.11 & -0.100 \\
PV scale factor & 0.020 & 0.020 & 0.020 \\
Jet scale factor & -0.12 & -0.100 & -0.090 \\
Taggability scale factor & -0.11 & -0.100 & -0.090 \\
\hline Total signal prediction & 1.9 & 1.8 & 1.7 \\
Total signal error & 0.36 & 0.34 & 0.32 \\
Relative signal error & 0.18 & 0.19 & 0.19 \\
Signal acceptance & $4.7 \%$ & $4.4 \%$ & $4.1 \%$ \\
Acceptance error & $0.86 \%$ & $0.83 \%$ & $0.77 \%$ \\
\hline
\end{tabular}

Table D.38: Evolution of the systematic uncertainties on the signal with each selection requirement. The assumed masses are $M_{\rho}=260 \mathrm{GeV} / c^{2}$, $M_{\pi}=140 \mathrm{GeV} / c^{2}$, with $M_{V}=100 \mathrm{GeV}$. 


\begin{tabular}{|l|lll|}
\hline sample & $m_{j j}$ & $m_{W j j}$ & $\Delta \phi(j, j)$ \\
\hline \hline Non- $W / Z$ BG Estimate & 1.1 & 0.91 & 0.60 \\
Cross sections & 0.90 & 0.76 & 0.67 \\
MC statistics & 0.30 & 0.28 & 0.29 \\
Diboson kinematics & 0.001 & 0 & 0 \\
Jet energy scale & -0.45 & -0.040 & -0.020 \\
$b / c$-jet TRF's & 0.62 & 0.53 & 0.49 \\
Mistag TRF's & -0.22 & -0.19 & -0.17 \\
Jet smearing & -0.96 & -1.1 & -1.2 \\
Track smearing & -0.14 & -0.55 & -0.60 \\
PV scale factor & -0.27 & -0.27 & -0.43 \\
Jet scale factor & -0.82 & -1.1 & -1.1 \\
Taggability scale factor & -0.66 & -0.95 & -1.1 \\
\hline Total BG prediction & 12 & 10 & 8.9 \\
Total BG error & 2.2 & 2.4 & 2.4 \\
\hline
\end{tabular}

Table D.39: Evolution of the systematic uncertainties on the backround with the final selection requirements, optimized for for $M_{\rho}=275 \mathrm{GeV} / c^{2}$, $M_{\pi}=150 \mathrm{GeV} / c^{2}$.

\begin{tabular}{|l|lll|}
\hline sample & $m_{j j}$ & $m_{W j j}$ & $\Delta \phi(j, j)$ \\
\hline \hline MC statistics & 0.033 & 0.032 & 0.032 \\
Jet energy scale & 0.080 & 0.030 & 0.040 \\
$b / c$-jet TRF's & 0.13 & 0.12 & 0.11 \\
Jet smearing & -0.21 & -0.23 & -0.22 \\
Track smearing & -0.040 & -0.070 & -0.060 \\
PV scale factor & 0.080 & 0.070 & 0.070 \\
Jet scale factor & -0.050 & -0.030 & -0.020 \\
Taggability scale factor & -0.050 & -0.040 & -0.030 \\
\hline Total signal prediction & 1.9 & 1.7 & 1.6 \\
Total signal error & 0.29 & 0.29 & 0.27 \\
Relative signal error & 0.15 & 0.17 & 0.16 \\
Signal acceptance & $5.4 \%$ & $5.0 \%$ & $4.8 \%$ \\
Acceptance error & $0.83 \%$ & $0.83 \%$ & $0.78 \%$ \\
\hline
\end{tabular}

Table D.40: Evolution of the systematic uncertainties on the signal with each selection requirement. The assumed masses are $M_{\rho}=275 \mathrm{GeV} / c^{2}$, $M_{\pi}=150 \mathrm{GeV} / c^{2}$, with $M_{V}=100 \mathrm{GeV}$. 


\section{Bibliography}

[1] Quarks and Leptons: An Introductory Course in Modern Particle Physics, F. Halzen and A. Martin, John Wiley and Sons Inc. (1984).

[2] The Particle Data Group, Phys. Lett. B 592, 1 (2004)

[3] T.D. Lee and C.N. Yang, Phys. Rev., 104254 (1956).

[4] C.S. Wu et al., Phys. Rev., 1051413 (1957).

[5] Introduction to Elementary Particles, D. Griffiths, John Wiley and Sons Inc. (1987)

[6] Gauge Theories of the Strong, Weak and Electromagnetic Interactions, C. Quigg, Addison Wesley Longman, Inc. (1983).

[7] P. Higgs, Phys. Rev. Lett. 13508 (1964).

[8] The LEP Electroweak Working Group, http://lepewwg.web.cern.ch/ LEPEWWG/.

[9] The ALEPH, DELPHI, L3 and OPAL Collborations, and the LEP Working Group for the Higgs Boson, Phys. Lett. B 56561 (2003).

[10] "Two Lectures on Technicolor," K. Lane (26 Feb. 2002); hep-ph/0202255.

[11] C. Hill and E. Simmons, Phys. Rept. 381235 (2003); Erratum-ibid. 390 553 (2004); hep-ph/0203079.

[12] "A Combination of CDF and DØ Results on the Mass of the Top Quark," The Tevatron Electroweak Working Group (20 Mar. 2006); hepex/0603039.

[13] K. Lane and S. Mrenna, Phys.Rev. D 67115011 (2003); hep-ph/0210299.

[14] T. Affolder, et al., Phys. Rev. Lett. 841110 (2000). 
[15] F. Abe, et al., Phys. Rev. Lett. 83, 3124 (1998).

[16] V. Abazov et al., Phys. Rev. Lett. 87, 61802 (2001).

[17] J. Abdallah, et al., Eur. Phys. C 22, 17 (2001).

[18] "A Search for Techniparticle Production at DØ Run II in the Mode $\rho_{T} \rightarrow$ $W(\rightarrow e \nu)+\pi_{T}(\rightarrow b \bar{b}(\bar{c}))$ with b-quark Identification Using Secondary Vertex Reconstruction," L. Feligioni and M. Narain, DØ Note 4967 (25 Apr. 2006).

[19] M. Narain, private communication (4 Apr. 2006).

[20] "The Tevatron Run II Handbook," D. McGinnis, http://www-bd.fnal. gov/runII/index.html.

[21] A. Heinson, http://www-d0.fnal.gov/Run2Physics/WWW/drawings/ dodet_tracking_ah.eps.

[22] V. M. Abazov et al., [The DØ Collaboration], accepted by Nucl. Instr. and Methods; hep-physics/0507191, Fermilab-Pub-05/341-E.

[23] S. Abachi et al., Nucl. Instrum. Methods Phys. Res. A 338, 185 (1994); Fermilab-Pub-93/179-E.

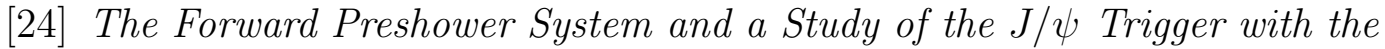
DØ Detector, A. Patwa, Ph.D. dissertation, State University of New York at Stony Brook, Stony Brook, New York, USA, May 2002.

[25] "Ordering a Chaos or... Technical Details of AA Tracking," G. Borissov, All DØ Mtg (28 Feb 2003), http://www-d0.fnal.gov/atwork/adm/d0_ private/2003-02-28/adm_talk.ps.

[26] "HTF: Histogramming Method for Finding Tracks. The Algorithm Description," A. Khanov, DØ Note 3778 (23 Sep. 2000).

[27] "Primary Vertex Reconstruction in p14," A. Garcia-Bellido et al., DØ Note 4320 (7 Jan. 2004).

[28] "Probabilistic Primary Vertex Selection," A. Schwartzman and M. Narain, DØ Note 4042, (8 Nov. 2002).

[29] "Run II Jet Physics," G. Blazey et al., (10 May 2000); hep-ex/0005012. 
[30] "Jet Algorithms in the DØ RunII Software: Description and Users Guide," E. Busato and B. Andrieu, DØ Note 4457, 13 August 2004.

[31] "NADA: A New Event by Event Hot Cell Killer," B. Olivier et al., DØNote 3687 (28 Jul. 2000).

[32] See the CalNada.rcp file in the p14.06.01 version of the DØ cal_nada CVS package.

[33] "Towards a Coherent Treatment of Calorimetric Energies: Missing Transverse Energy, Jets, E.M. Objects and the T42 Algorithm," U. Bassler and G. Bernardi, DØ Note 4124 (24 Mar. 2003).

[34] "Improvements from the T42 Algorithm on Calorimeter Objects Reconstruction," G. Bernardi, E Bustato and J.R. Vlimant, DØ Note 4335 (26 Jan. 2004).

[35] "Measurement of the t⿱亠䒑 Production Cross-Section at $\sqrt{s}=1.96 \mathrm{TeV}$ in the Muon+Jets Final State using a Topological Method," T. Golling, DØ Note 4667 (16 Dec. 2004).

[36] "Jet Energy Scale at DØ RunII," J.L. Agram et al., DØNote 4720 (15 Feb. 2005).

[37] "Muon Segment Algorithm," O. Peters, http://www-d0.fnal.gov/ nikhef/muon_reco/segmentreco.

[38] "The Fit Algorithm in muo_trackreco," F. Deliot, http://www-d0.fnal. gov/ deliot/fitalg.ps.

[39] "Parametrizing the Local Muon Resolution in p13 Data for PMCS," A. Askew, DØ Note 4314 (29 Dec. 2003).

[40] "Track Parameter Error Matrix Propagation in Matter and Magnetic Field, Error Matrices Combination," L. Chevalier et al., http:// www-clued0.fnal.gov/ ${ }^{\sim}$ tuchming/myprop.ps, (24 Oct. 2001).

[41] "Content of the p13 Muon Thumbnail," DØ Muon Algorithm and Identification Groups, http://www-clued0.fnal.gov/ ${ }^{\sim}$ clement/muons/p13/ mu_tmb_doc.ps, (3 Feb. 2003).

[42] http://www-d0.fnal.gov/phys_id/muon_id/d0_private/certif/ p14/index.html 
[43] A Search for Techniparticles at DØ RunII, L. Feligioni, Ph.D. dissertation, Boston University, Boston, MA (2006).

[44] "Electron Likelihood in p14," J. Kozminski et al., DØ Note 4449 (5 Apr. 2004).

[45] T. Sjostrand et al., Comp. Phys. Comm. 135, 238 (2001); hep$\mathrm{ph} / 0010017$,

[46] J. Campbell and K. Ellis, http://mcfm.fnal.gov

[47] The CTEQ collaboration, H. Lai, et al., Eur.Phys.J. C 12375 (2000); hep-ph/9903282.

[48] A. Pukhov et al., (6 Aug 1999); hep-ph/9908288.

[49] M. Mangano et al., JHEP 0307001 (2003); hep-ph/0206293

[50] M. Mangano, http://cepa.fnal.gov/patriot/mc4run2/MCTuning/ 061104/mlm.pdf (11 Jun. 2004).

[51] "Search for $W H \rightarrow l \nu b \bar{b}, "$ P. Tamburello and D. Meder, DØ Note 4486 (5 Aug. 2004).

[52] P. Tamburello, private communication (1 May 2006).

[53] T. Golling, private communications (30 Jun. 2004 and 1 Jul. 2004).

[54] "Performance of the JLIP b-tagger in p14," D. Bloch et al., D $\varnothing$ Note 4348 (30 Mar. 2004).

[55] "Update of the JLIP b-tagger Performance in p14/pass2 with JES 5.3," D. Bloch and B Clement, DØ Note 4824 (23 Jun. 2005).

[56] Fit functions and parameter values were obtained from examination of the TRF_JLIP_p14pass2.root in the DØ CVS package dØroot_jlip, version v00-00-21.

[57] "System D or How to Get Signal Backgrounds and Their Efficiencies from Data," B. Clement et al., DØ Note 4159 (11 Jun. 2003).

[58] J. Campbell and K. Ellis, Phys. Rev. D 65113007 (2002); hepph/0202176. 
[59] "The NNLO Predictions for the Rates of the $W / Z$ Production in $p \bar{p}$ Collisions," S. Alekhin, hep-ph/0307219 (17 Jul. 2003).

[60] J. Campbell and K. Ellis, Phys. Rev. D 60113006 (1999); hep$\mathrm{ph} / 9905386$.

[61] "Search for Higgs Boson in $H \rightarrow W W^{(*)} \rightarrow l^{+} \nu l^{-} \nu$ decays at D $\varnothing$ in Run II,” J. Elmsheuser and M. Hohlfeld, DØ Note 4759 (25 May 2005).

[62] N. Kidonakis and R. Vogt, Phys.Rev. D 68114014 (2003); hep$\mathrm{ph} / 0308222$.

[63] "Top Quark Production Dynamics," E. Lanen (4 Aug 2003); hep$\mathrm{ph} / 0308025$.

[64] See the D $\varnothing$ CVS package limit_calculators.

[65] "A Recipe for the Construction of Confidence Limits, "I. Bertram et al., DØ Note 3476 (7 Sep. 1999).

[66] "Calculating Confidence Limits," J. Linnemann et al., DØ Note 4491, (1 Jul. 2004).

[67] "A Search for Techniparticle Production at $\sqrt{s}=1.96 \mathrm{TeV}$ in $\rho_{T} \rightarrow$ $W(\rightarrow e \nu)+\pi_{T}(\rightarrow b \bar{b}(\bar{c}))$ Using Neural Network," D. Choo et al., D Note 5125 (14 Jun. 2006).

[68] "A Search for $W H$ production at $\sqrt{s}=1.96 \mathrm{TeV}$." The D $\varnothing$ Collaboration, DØ Note 5054-CONF (19 Mar. 2006). 